\title{
Improving time series modeling by decomposing and analyzing stochastic and deterministic influences
}

Ricardo Araújo Rios 

Modelagem de séries temporais por meio da decomposição e análise de influências estocásticas e determinísticas

Ricardo Araújo Rios 

SERVIÇO DE PÓS-GRADUAÇÃO DO ICMC-USP

Data de Depósito:

Assinatura:

\title{
Improving time series modeling by decomposing and analyzing stochastic and deterministic influences
}

\author{
Ricardo Araújo Rios
}

Advisor: Prof. Dr. Rodrigo Fernandes de Mello

Doctoral dissertation submitted to the Instituto de Ciências Matemáticas e de Computação - ICMC-USP, in partial fulfillment of the requirements for the degree of the Doctorate Program in Computer Science and Computational Mathematics. FINAL VERSION. 
Ficha catalográfica elaborada pela Biblioteca Prof. Achille Bassi e Seção Técnica de Informática, ICMC/USP, com os dados fornecidos pelo(a) autor(a)

R586i Rios, Ricardo Araújo
Improving time series modeling by decomposing
and analyzing stochastic and deterministic
influences / Ricardo Araújo Rios; orientador
Rodrigo Fernandes de Mello. -- São Carlos, 2013.
133 p.
Tese (Doutorado - Programa de Pós-Graduação em
Ciências de Computação e Matemática Computacional) --
Instituto de Ciências Matemáticas e de Computação,
Universidade de São Paulo, 2013.
1. time series analysis. 2. decomposition. 3.
additive noise. 4. stochastic and deterministic
influences. 5. Empirical Mode Decomposition. I.
Mello, Rodrigo Fernandes de, orient. II. Título.


SERVIÇO DE PÓS-GRADUAÇÃO DO ICMC-USP

Data de Depósito:

Assinatura:

\section{Modelagem de séries temporais por meio da decomposição e análise de influências estocásticas e determinísticas}

\section{Ricardo Araújo Rios}

Orientador: Prof. Dr. Rodrigo Fernandes de Mello

Tese apresentada ao Instituto de Ciências Matemáticas e de Computação - ICMC-USP, como parte dos requisitos para obtenção do título de Doutor em Ciências - Ciências de Computação e Matemática Computacional. VERSÃO REVISADA. 
Ficha catalográfica elaborada pela Biblioteca Prof. Achille Bassi e Seção Técnica de Informática, ICMC/USP, com os dados fornecidos pelo(a) autor(a)

\section{Rios, Ricardo Araújo}

Melhoria da modelagem de séries temporais pela decomposição e análise de influências estocásticas e determinísticas / Ricardo Araújo Rios; orientador Rodrigo Fernandes de Mello. -- São Carlos, 2013. $133 \mathrm{p}$.

Tese (Doutorado - Programa de Pós-Graduação em Ciências de Computação e Matemática Computacional) -Instituto de Ciências Matemáticas e de Computação, Universidade de São Paulo, 2013.

1. análise de séries temporais. 2. decomposição. 3. ruído aditivo. 4. influências estocásticas e determinísticas. 5. Decomposição de Modo Empírico. I. Mello, Rodrigo Fernandes de, orient. II. Título. 


\section{Acknowledgments}

First of all, I would like to offer my gratitude to God, who has been blessing me every single day. I also wish to express my sincere gratitude to my advisor, professor Rodrigo Mello. During the last years, he was more than a formal advisor, helping me to understand new concepts and working hard to produce this work. Thank you very much, Rodrigo, for showing me the importance of researching without having numbers as parameter. For sure, our collaboration and friendship will never end.

Well, I was impatient to start talking about my wife and my daughter. Tati, you are my strength, my motivation, and my inspiration. Thank you very much for your patience and love. You are present in every page of this thesis. Thank you very much for making my days and trips funnier. Mari, my little girl, you came up a few months ago, but I cannot even remeber my life before you. You suddenly became the main reason for everything. Your tender smile moves me and makes me forget any tough situation.

I am deeply grateful to my father Robério, my mother Josinha, my brothers Guga, Joyce (Guga's wife and my stepsister :]), and Bebel. You are the greatest models for me. I am pretty sure you are always by my side, regardless the situation I know I can count on you. I love you so much. My grandfather Alcides and my grandmother Isabel, you started all this mess when you left behind a quiet life in the countryside to live in a bigger city and give better conditions to your children. I am forever grateful to you. I would like to thank my new family Valdeci, Dilzete, Tiago, Eva e Rodrigo for being always there sharing amazing moments.

Professor Michall Small, thank you very much for your help and support during my stay in Hong Kong. You allowed me to develop a new research line and to try out one of the most incredible experience of my life. The memories from HK will never leave my head. Thank you also Ruoxi Xiang, Xiaoke $\mathrm{Xu}$, and Liu Xiaofan for our chats that helped me during the adaptation process and made me to learn and respect your culture even more.

I also thank Professor Lael Parrott for your help while I was in Montreal, for your ded- 
ication during our meetings and all support after my return to Brazil. I am also thankful to Gillaume Latombe, Phillipe Girard, Clément Chion, Caroline Von Schilling, Rodolphe Coulon, and Patrick Verhaar. You guys made my workdays more pleasant with our coffee breaks.

My dear friends Pedro, Taynara, Sophie, Leo, Aninha, Lucas, Natalia, Théo, you helped me a lot during the process of settling down. You guys were/are our family in Montreal. I have learnt a lot from you and I will never forget every moment shared with you (Does this call mean something?). Afshin, Mme Yanick and Mme Marie, «je vous remercie beaucoupe pour m'aider avec le frances!» I will never forget you. Mr. Richard Lancing, your blessing messages every sunday morning gave me peace and strength. Thank you very much.

My sincere thanks to my friends Renato Ishii, Marcelo Albertini, Cássio Martini, Paulo Henrique Gabriel and Fausto Guzzo who directly contributed with this thesis during our chats and presentations. My dear friend Marcos Cintra, you were my teacher and now I am sure our friendship goes on for many years. Daniele Jacinto, thank you very much for your help and support several times during this time. I have no words to express my gratitude to you. Vinícius Durelli, thank you very much for your help during the final preparation of this thesis and for helping me to produce the Appendix A. My dear friend José Rubens, thanks for sharing nice moments playing soccer. I already miss our goals. Professor Dorival Leão, thanks for our chats that helped me a lot during this process. I also thank all my friends and collegues from Maiti-Roliudi (Cássio Prazeres, Maycon Leone, Leonardo Barreto, Gabriel Gadelha, Tácito Neves, Fernando Panont, Danilo Coimbra, José Augusto Martins, and David Neto), from Biocom, from Estatcamp and a list of friends that's really worth mentioning: Leonardo Botega e Cacau, Simone Borges, Matheus Viana, James Santos, Débora Corrêa, Kamila Rios, Bruno Kimura, and Paulo Cereda.

Finally, I also wish to express a special gratitude to Graciano Amed (Chano) and Evgueni Dodonov. Although you guys have left us extremely soon, your dedication to work inspired me a lot.

This research was supported by FAPESP (Sao Paulo Research Foundation), Brazil, under grant 2009/18293-9 and CAPES (Coordination for Enhancement of Higher Education Personnel) under grant 5983-11-8. 


\section{Abstract}

This thesis presents a study on time series analysis, which was conducted based on the following hypothesis: time series influenced by additive noise can be decomposed into stochastic and deterministic components in which individual models permit obtaining a hybrid one that improves accuracy. This hypothesis was confirmed in two steps. In the first one, we developed a formal analysis using the Nyquist-Shannon sampling theorem, proving Intrinsic Mode Functions (IMFs) extracted from the Empirical Mode Decomposition (EMD) method can be combined, according to their frequency intensities, to form stochastic and deterministic components. Considering this proof, we designed two approaches to decompose time series, which were evaluated in synthetic and real-world scenarios. Experimental results confirmed the importance of decomposing time series and individually modeling the deterministic and stochastic components, proving the second part of our hypothesis. Furthermore, we noticed the individual analysis of both components plays an important role in detecting patterns and extracting implicit information from time series. In addition to these approaches, this thesis also presents two new measurements. The first one is used to evaluate the accuracy of time series modeling in forecasting observations. This measurement was motivated by the fact that existing measurements only consider the perfect match between expected and predicted values. This new measurement overcomes this issue by also analyzing the global time series behavior. The second measurement presented important results to assess the influence of the deterministic and stochastic components on time series observations, supporting the decomposition process. Finally, this thesis also presents a Systematic Literature Review, which collected important information on related work, and two new methods to produce surrogate data, which permit investigating the presence of linear and nonlinear Gaussian processes in time series, irrespective of the influence of nonstationary behavior. 


\section{Resumo}

Esta tese apresenta um estudo sobre análise de séries temporais, a qual foi conduzida baseada na seguinte hipótese: séries temporais influenciadas por ruído aditivo podem ser decompostas em componentes estocásticos e determinísticos que ao serem modelados individualmente permitem obter um modelo híbrido de maior acurácia. Essa hipótese foi confirmada em duas etapas. Na primeira, desenvolveu-se uma análise formal usando o teorema de amostragem proposto por Nyquist-Shannon, provando que IMFs (Intrinsic Mode Functions) extraídas pelo método EMD (Empirical Mode Decomposition) podem ser combinadas de acordo com suas intensidades de frequência para formar os componentes estocásticos e determinísticos. Considerando essa prova, duas abordagens de decomposição de séries foram desenvolvidas e avaliadas em aplicações sintéticas e reais. Resultados experimentais confirmaram a importância de decompor séries temporais e modelar seus componentes estocásticos e determinísticos, provando a segunda parte da hipótese. Além disso, notou-se que a análise individual desses componentes possibilita detectar padrões e extrair importantes informações implícitas em séries temporais. Essa tese apresenta ainda duas novas medidas. A primeira é usada para avaliar a acurácia de modelos utilizados para predizer observações. A principal vantagem dessa medida em relação às existentes é a possibilidade de avaliar os valores individuais de predição e o comportamento global entre as observações preditas e experadas. A segunda medida permite avaliar a influência dos componentes estocásticos e determinísticos sobre as séries temporais. Finalmente, essa tese apresenta ainda resultados obtidos por meio de uma revisão sistemática da literatura, a qual coletou importantes trabalhos relacionados, e dois novos métodos para geração de dados substitutos, permitindo investigar a presença de processos Gaussianos lineares e não-lineares, independente da influência de comportamento não-estacionário. 


\section{Contents}

$\begin{array}{lc}\text { List of Figures } & \mathbf{x i}\end{array}$

$\begin{array}{ll}\text { List of Tables } & \text { xvii }\end{array}$

$\begin{array}{ll}\text { List of Algorithms } & \text { xix }\end{array}$

$\begin{array}{ll}\text { List of Abbreviations } & \text { xxi }\end{array}$

1 Introduction $\quad 1$

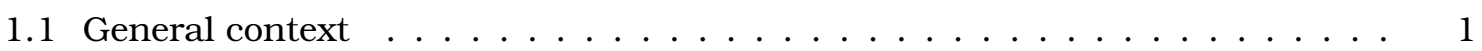

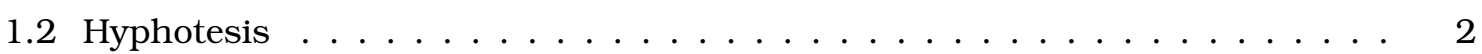

2 Time Series Decomposition $\quad 7$

2.1 Introduction .......................... 7

2.2 Decomposition methods ........................ 11

2.3 Concluding remarks . . . . . . . . . . . . . . . . . . . 15

3 Formal Definition On Time Series Decomposition $\quad 17$

3.1 Introduction ......................... 17

3.2 On the Nyquist-Shannon sampling theorem . . . . . . . . . . . . . . 18

3.3 Analyzing frequencies of Intrinsic Mode Functions . . . . . . . . . . . . . . . . 19

3.4 Simulation results . . . . . . . . . . . . . . . . . . . . . 23 


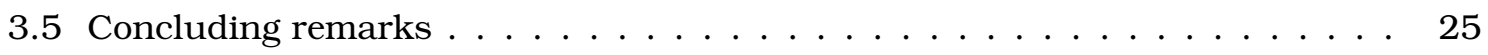

4 Approaches Proposed to Decompose Time Series $\quad 27$

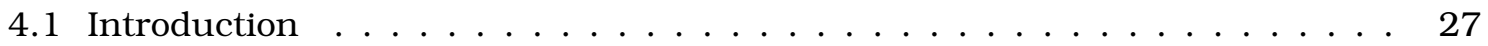

4.2 Empirical Mode Decomposition and Recurrence Plot: First Approach . . . . . 28

4.3 Empirical Mode Decomposition and Mutual Information: The Second Approach 33 4.3.1 The proposed decomposition approach . . . . . . . . . . . . 36

4.3 .2 Determinism rate . . . . . . . . . . . . . . . . . . 38

4.4 Concluding remarks . . . . . . . . . . . . . . . . . . . 40

5 Experimental Results $\quad 43$

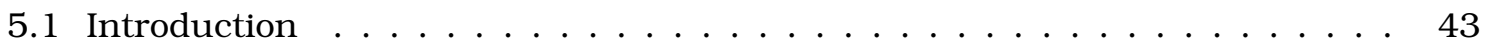

5.2 Experimental setup $\ldots \ldots \ldots \ldots \ldots \ldots \ldots \ldots$

5.3 MDDL measurement $\ldots \ldots \ldots \ldots \ldots \ldots \ldots \ldots$

5.4 Scenario I: Sine function plus white noise $\ldots \ldots \ldots \ldots \ldots \ldots$

5.5 Scenario II: Sine function plus ARMA and colored noise . . . . . . . . . . . . 54

5.6 Scenario III: Lorenz system plus white noise . . . . . . . . . . . . . . 60

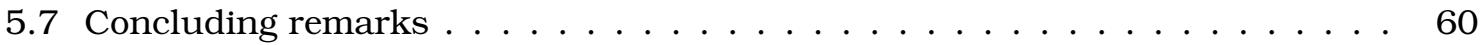

6 Real-World Application $\quad 65$

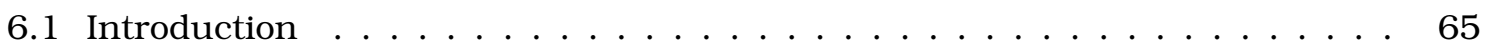

6.2 Experimental evaluation $\ldots \ldots \ldots \ldots \ldots \ldots \ldots$

6.3 Results . . . . . . . . . . . . . . . . . . . . . 69

6.3 .1 Dataset: FAPAR . . . . . . . . . . . . . . . 69

6.3 .2 Dataset: Rainfall . . . . . . . . . . . . . . . . . . 70

6.3.3 Dataset: Temperature . . . . . . . . . . . . . . . . 71

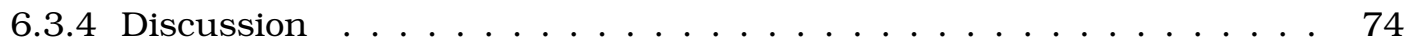

6.4 Concluding remarks $\ldots \ldots \ldots \ldots \ldots \ldots \ldots \ldots$

$\begin{array}{lll}7 \text { Conclusions } & 77\end{array}$

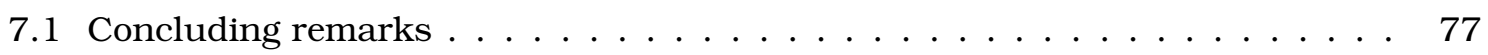

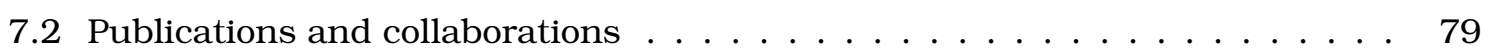

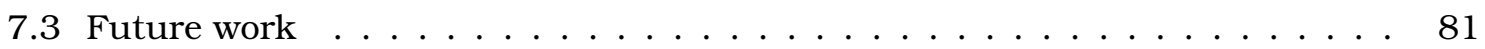




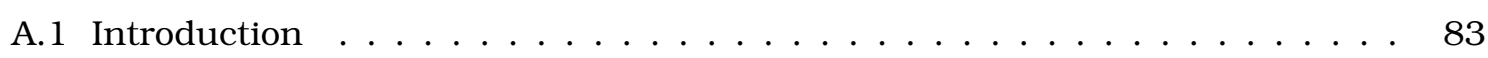

A.2 Systematic Review . . . . . . . . . . . . . . . . . . . 84

A.3 Phase I: Search . . . . . . . . . . . . . . . . . . . . . . 85

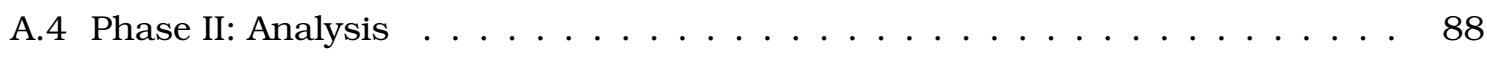

A.5 Phase III: Conclusion $\ldots \ldots \ldots \ldots$. . . . . . . . . . . . . . . 93

$\begin{array}{ll}\text { B Dynamical System Background } & 95\end{array}$

$\begin{array}{ll}\text { C Surrogate Data } & 103\end{array}$

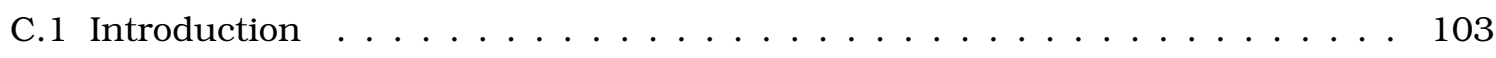

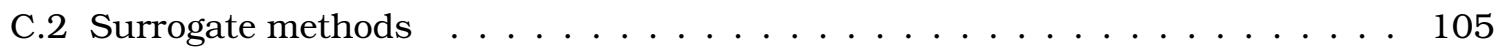

C.3 Proposed surrogate methods . . . . . . . . . . . . . . . . . . . . . 109

C.4 Analyzing the proposed surrogate methods $\ldots \ldots \ldots \ldots \ldots \ldots$

C.5 Experimental results . . . . . . . . . . . . . . . . . . . 114

C.5.1 Synthetic Time Series . . . . . . . . . . . . . . . . 116

C.5.2 Real-World Time Series . . . . . . . . . . . . . . . . . . . 118

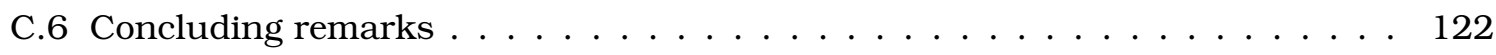

$\begin{array}{ll}\text { Referências } & 133\end{array}$ 


\section{List of Figures}

2.1 Examples of continuous and discrete time series. The discrete time series was sampled from the continuous one. . . . . . . . . . . . . . . . . 8

2.2 Example of a forecasting model: $\circ$ and $*$ represent the past and future observations, respectively (adapted from (Box et al., 1994)). . . . . . . . . . . . . . 9

2.3 The original time series is represented by the dotted line in all plots. (a) The maxima and minima observations are selected. $(b-c)$ The cubic spline method is applied to the maxima and the minima, connecting all observations and producing the upper and lower envelopes, respectivelly. (d) The mean envelope is obtained by calculating the mean values between the upper and lower envelopes. . . . . . . . . . . . . . . . . . . . . . . . .

2.4 The (a) deterministic, $\sin (2 \pi t)$, and (b) stochastic, $\varepsilon(0,4)$, components used to create the noisy time series $x(t) \ldots \ldots \ldots \ldots \ldots \ldots$

2.5 $\operatorname{IMFs}\left(h_{n}(t)\right)$ and residue $(r(t))$ extracted from a noisy time series $x(t)=\sin (2 \pi t)+$ $\varepsilon(0,1)$. The $x$-axis represents the time in all plots. . . . . . . . . . . . 1

3.1 The noisy time series $x(t)=\sin (2 \pi t)+\varepsilon(0,1)$ is shown in plot (a). Plots from (b) to (i) show all IMFs $h_{n}(t)$ extracted at each iteration from the time series $x(t)$ using the EMD method. The last plot (j) shows the residue $r(t)$. The $x$-axis represents the time in all plots. 
3.2 Periodogram for each extracted IMF. The vertical dotted line represents the mean weighted frequency $\overline{\mathcal{F}}(\cdot)$ defined in Equation 3.12. By considering these plots, we notice the maximum frequency is reduced as new IMFs are iteratively extracted. . . . . . . . . . . . . . . . . . . . .

4.1 (a) Noisy time series created considering the stochastic and deterministic components as presented in Figure 2.4. (b) The dotted line represents the deterministic component $(\sin (2 \pi t))$ used to create the noisy time series. The deterministic component estimated by combining the IMFs extracted using EMD is represented by the continuous line. . . . . . . . . . . . . . . . .

4.2 Recurrence plot for the first four IMFs. By considering these RP structures, we notice the influence of isolated points, indicating the presence of stochastic

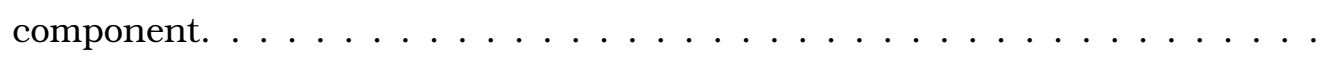

4.3 Recurrence plot for the last four IMFs. By considering these RP structures, we notice the influence of isolated points reduces as the last IMFs are extracted from the time series, whereas the diagonal lines and recurrent patterns increases, indicating the presence of deterministic influence. . . . . . . . . .

4.4 (a) Stochastic component created by the IMFs from 1 to 4 . (b) Recurrence plot for the stochastic component. (c) Deterministic component created by the IMFs from 5 to 8. (d) Recurrence plot for the deterministic component. . . . .

4.5 Example of the stochastic influence on a time series: (a) $x(t)$ is influenced by a positive noise; $(\mathrm{b}) \mathrm{x}(\mathrm{t})$ is influenced by a negative noise. . . . . . . . . .

5.1 The continuous curve represents the expected sine values $x(t)$, and the other two curves represent the predicted time series $p_{1}(t)$ and $p_{2}(t)$ (adapted from (Rios and Mello, 2013)). . . . . . . . . . . . . . . . . . . . . . . . 
5.2 The MDDL plot shows the differences of the expected time series $x(t)$ in relation to: (a) the predicted noisy observations $p_{1}(t)$; and (b) mean observations $p_{2}(t)$. The solid line represents the warping path and the diagonal line is represented by the dashed one. By looking at these plots, it is possible to notice the shaded area in the first case is much smaller, indicating that the model used to predict $p_{1}(t)$ is better than the one used to predict $p_{2}(t)$ (adapted from (Rios and Mello, 2013)). . . . . . . . . . . . . . . . . . . . 48

5.3 (a) Time series generated by sine function. (b) The sine time series unfolded in phase space. (c) The noisy time series generated by sine function plus the white noise process. (d) The noisy time series unfolded in phase space. . . . .

5.4 Considering the qqplot and boxplot charts, one can notice the stochastic component strongly influenced the general behavior of the sine time series. . . . . 50

5.5 (a) The deterministic component (plot in the middle), obtained after decomposition, plotted against the undecomposed sinusoidal noisy time series (upper plot) and the expected behavior (lower plot). These series were shifted in $y$-axis in order to improve visualization. (b) The deterministic component reconstructed in the phase space. . . . . . . . . . . . . . . . . 51

5.6 (a) RBF-based predicted observations unfolded in phase space. (b) Polynomialbased predicted observations unfolded in phase space. . . . . . . . . . . 52

5.7 MDDL plot for the predicted and expected observations using (a) RBF, (b) Polynomial, and (c) AR models on the decomposed components. . . . . . . . . 53

5.8 MDDL plot for the predicted and expected observations using the hybrid approaches: (a) RBF/AR, and (b) Polynomial/AR models. . . . . . . . . . . . .

5.9 MDDL plot for the predicted and expected observations using RBF (a), Polynomial (b), and AR (c) models without decomposition. . . . . . . . . . . .

5.10 Comparison among the different prediction approaches: (a) RBF, Polynomial and AR models adjusted on the decomposed components; (b) hybrid models RBF/AR and Polynomial/AR adjusted on the decomposed components; (c) RBF, Polynomial and AR models directly applied on the undecomposed time series. 
5.11 (a) Sine plus autoregressive moving average time series. (b) Sine plus autoregressive moving average time series unfolded in phase space. (c) The deterministic component. (d) The deterministic component unfolded in phase

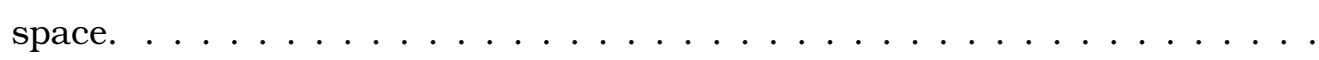

5.12 (a) Sine plus colored-noise time series. (b) Sine plus colored-noise time series unfolded in phase space. (c) The deterministic component. (d) The deterministic component unfolded in phase space. . . . . . . . . . . . . . . 59

5.13 Lorenz time series influenced by different stochastic components. . . . . . . . 62

6.1 2D World maps to illustrate the FAPAR dataset. . . . . . . . . . . . . . 68

6.2 Six 2D World maps to illustrate the Rainfall dataset. . . . . . . . . . . . . . 68

6.3 Six 2D World maps to illustrate the Temperature dataset. . . . . . . . . . . 69

6.4 Determinism map obtained with EMD-MI to estimate determinism rates on FAPAR dataset. . . . . . . . . . . . . . . . . . . 70

6.5 Deterministic 2D World map obtained with EMD-MI on Rainfall dataset. . . . 71

6.6 Binary 2D World maps obtained after applying the determinism rate on FAPAR and Rainfall datasets. . . . . . . . . . . . . . . . . . . . . 72

6.7 Deterministic 2D World map obtained with EMD-MI on Temperature dataset. 73

6.8 Global air masses. Figure provided by NASA under public domain license. . . 73

6.9 A sample of original time series selected from FAPAR (a) and Rainfall (b) datasets. We observe their dynamics are completely different, although they have similar stochastic characteristics. . . . . . . . . . . . . . . . 75

B.1 Time series produced by the Lorenz system. . . . . . . . . . . . . . . . . 98

B.2 Estimating the delay dimension using AMI. . . . . . . . . . . . . . . 98

B.3 Estimating the embedded dimension using FNN . . . . . . . . . . . . . . 99

B.4 Lorenz system: time series unfolded into phase space. . . . . . . . . . . . . . 101

C.1 Time series used to evaluate the proposed methods: (a) A sine function combined with a trend; (b) A white noise process combined with a trend; (c) $\mathrm{Au}-$ toregressive process combined with a trend; (d) Global Temperature (Shumway and Stoffer, 2006); (e) Atmospheric concentrations of CO2 (Cleveland, 1993); (f) Dow Jones Utilities Index (Brockwell and Davis, 2002). . . . . . . . . . . . 115 
C.2 Surrogates generated from the synthetic time series created by the combination of trend and sine function. The left side presents the original time series (red continuous line) and its surrogates (dashed lines). In the middle and right side, it is presented the ACF and AMI plots. . . . . . . . . . . . . 117

C.3 Surrogates generated from the synthetic time series created by the combination of trend and white noise process. The left side presents the original time series (red continuous line) and its surrogates (dashed lines). In the middle and right side, it is presented the ACF and AMI plots. . . . . . . . . . . . . . 118

C.4 Surrogates generated from the synthetic time series created by an autoregressive noise. The left side presents the original time series (red continuous line) and its surrogates (dashed lines). In the middle and right side, it is presented the ACF and AMI plots. . . . . . . . . . . . . . . . . . . . . . . . . . . . 119

C.5 Surrogates generated from the real-world time series composed of average values, yearly, collected of global temperatures. The left side presents the original time series (red continuous line) and its surrogates (dashed lines). In the middle and right side, it is presented the ACF and AMI plots. . . . . . . . 120

C.6 Surrogates generated from the real-world time series composed of atmospheric concentrations of $\mathrm{CO} 2$. The left side presents the original time series (red continuous line) and its surrogates (dashed lines). In the middle and right side, it is presented the ACF and AMI plots. . . . . . . . . . . . . . . . 121

C.7 Surrogates generated from the real-world time series of Dow Jones Utilities Index. The left side presents the original time series (red continuous line) and its surrogates (dashed lines). In the middle and right side, it is presented the ACF and AMI plots. . . . . . . . . . . . . . . . . . . 


\section{List of Tables}

3.1 Weighted mean frequencies for all IMFs illustrated in Figure 3.1. . . . . . . . 26

5.1 Results considering models adjusted on the time series created by the combination of a sine function and an ARMA model. . . . . . . . . . . . . . 58

5.2 Results considering models adjusted on the time series created by the combination of a sine function and a colored noise . . . . . . . . . . . . . 59

5.3 Results obtained with the models ajusted on the noisy time series created for the third scenario, considering the same three analysis depicted in Tables 5.1

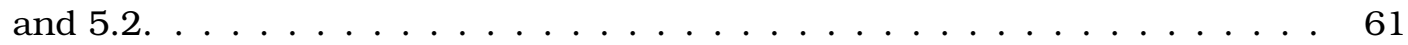

A.1 Number of papers returned by SLR. "Recovered papers" represents the number of papers returned by SLR, after removing invalid references. "Inclusion criteria" represents the number of papers selected after reading titles and abstracts. Finally, "Exclusion criteria" shows the number of remaining papers after being completely read. . . . . . . . . . . . . . . . . . . . 89

A.2 Frequency distribution of authors per country. . . . . . . . . . . . . . 89

A.3 List of authors who published more papers. . . . . . . . . . . . . . . . 90

A.4 Number of papers published per year. . . . . . . . . . . . . . . . 90

A.5 Relation between the number of papers and the publication type. . . . . . . 91

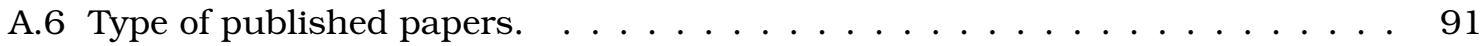


A.7 Goals of publications. . . . . . . . . . . . . . . . .

B.1 (a) Time series produced by the Lorenz system; (b) Time series unfolded into the phase space with embedded and delay dimensions equal to $m=3$ and $\tau=5$, respectively. . . . . . . . . . . . . . . . . . . . 100

C.1 Hypothesis test using Average Mutual Information (AMI): FT and AAFT rejected the null hypothesis in 9 out of 12 scenarios, confirming the surrogate series produced are not representative enough; EMD-FT and EMD-AAFT accepted the null hypothesis in all scenarios, confirming they produce more significative surrogate data. . . . . . . . . . . . . . . . . . . . 123 


\section{List of Algorithms}

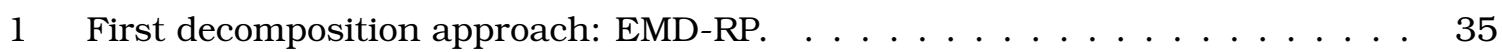

2 Second decomposition approach: EMD-MI . . . . . . . . . . . . . . 39 


\title{
List of Abbreviations
}

\author{
(Theiler's) AAFT Amplitude Adjusted Fourier Transformed \\ ACF \\ Autocorrelation Function \\ AMI \\ Average Mutual Information \\ AR \\ Autoregressive \\ ARMA \\ Autoregressive Moving Average \\ DTW \\ Dynamic Time Warping \\ DV \\ Darbellay and Vajda \\ EBM \\ Evidence-Based Medicine \\ EBSE \\ EEMD \\ EMD \\ Evidence-Based Software Engineering \\ Ensemble Empirical Mode Decomposition \\ Empirical Mode Decomposition \\ EMD-MI \\ Empirical Mode Decomposition and Mutual Information \\ EMD-RP \\ Empirical Mode Decomposition and Recurrence Plot \\ FAPAR \\ Fraction of Absorbed Photosynthesis Active Radiation \\ FFT \\ Fast Fourier Transform \\ FNN \\ False Nearest Neighbors \\ FT \\ Fourier Transform \\ (Theiler's) FT \\ Fourier Transformed - Surrogates (Appendix)
}


GEM

GP

GPCC

HSA

IAAFT

IES

iid

IMF

JRC

MDDL

MI

MODIS

NASA

PCA

PDF

POL

POL/AR

RBF

RBF/AR

RP

RQA

SD

SLR

SNR

SSA

SSS

STP

TFTS

WN

WT
Global Environment Monitoring

Grassberger-Procaccia

Global Precipitation Climatology Centre

Hilbert Spectral Analysis

Iterative Amplitude Adjusted Fourier Transformed

Institute for Environment and Sustainability

Independent and identically distributed

Intrinsic Mode Function

Joint Research Centre

Mean Distance from the Diagonal Line

Mutual Information

Moderate Resolution Imaging Spectroradiometer

National Aeronautics and Space Administration

Principal Component Analysis

Probability Density Function

Polynomial

Polynomial plus Autoregressive

Radial Basis Function

Radial Basis Function plus Autoregressive

Recurrence Plot

Recurrence Quantification Analysis

Spectral Density

Systematic Literature Review

Signal-to-noise ratio

Singular Spectrum Analysis

Small Shuffle Surrogate

Space-Time Separation Plot

Truncated Fourier Transform Surrogate

White Noise

Wavelet Transform 


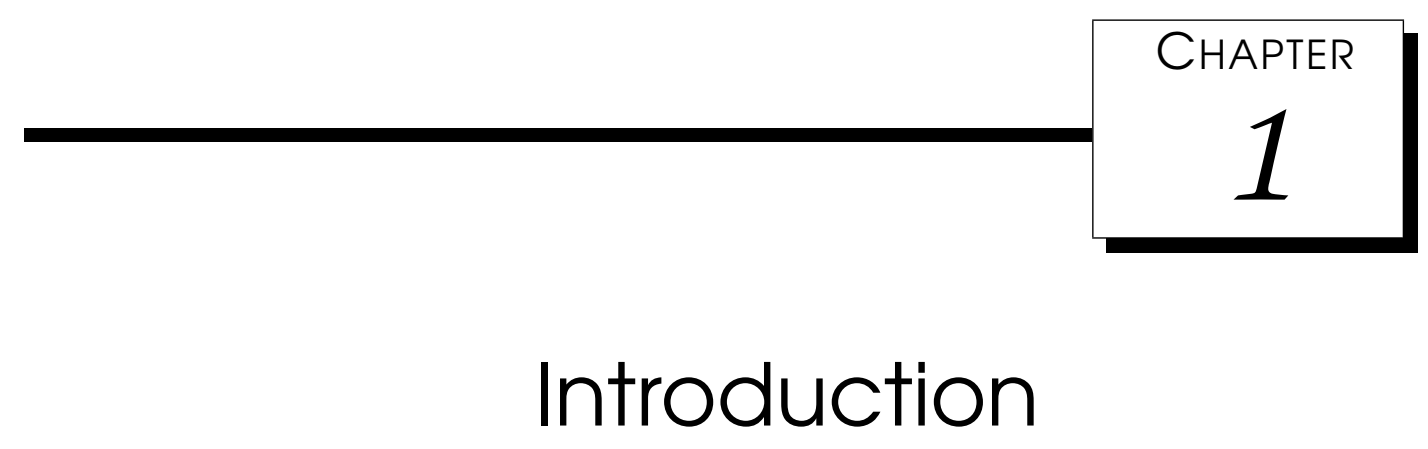

\subsection{General context}

The experimental data analysis area aims at designing methods to model and, consequently, understand system behavior (Shumway and Stoffer, 2006). According to Wangler and Backlund (2005); Zampa and Arnost (2002), systems are defined by a set of entities, named as components or subsystems, mutually connected. Those connections are responsible to define the different levels of relationships and dependencies among all components. By knowing the components and understanding their connections, one can model system behavior in order to improve decision making, forecasting, detection of failures, etc (Shumway and Stoffer, 2006).

In this sense, Wangler and Backlund (2005) suggest systems to be studied considering three well-defined steps. Firstly, a system may be separated into components to simplify its inherent complexity. Secondly, models are individually adjusted on every component and, finally, all models are combined to describe the global system behavior. In addition, those steps must also take into account data temporal dependencies, i.e., current system observations are related to past ones (Shumway and Stoffer, 2006). There are indeed 
situations in which data does not present dependencies, however many researchers tend to simplify every scenario to this baseline as discussed by Albertini (2012). They assume data is independent and identically distributed (iid), reducing modeling to the adjustment of probability density functions, such as Uniform and Normal, on data. As consequence, this approach imposes a strong bias that most certainly will risk data representation (Albertini, 2012).

The possibility of improving the analysis of time-dependent systems motivates this thesis whose hypothesis is detailed next.

\subsection{Hyphotesis}

Time series concepts are well established and essential when analyzing time-dependent systems. This area studies the behavior of systems considering temporal dependencies among observations in order to extract information and implicit data characteristics (Shumway and Stoffer, 2006). Two main branches are commonly adopted to perform this analysis: Dynamical Systems and Statistics. Dynamical Systems tools (Alligood et al., 1997) provide the best results when time series behavior is considered deterministic ${ }^{1}$. On the other hand, Statistical tools are indicated when time series present stochastic behavior $^{2}$.

Time series collected from real-world systems usually present a mixture of both components, i.e., the value of a single observation is influenced by deterministic and stochastic components. Consequently, modeling both components is essential to produce high accuracy results, once the discard of any component tends to depreciate results (Han and Liu, 2009), i.e., by only applying Dynamical System tools one may obtain malformed attractors, whereas only using Statistical tools one tends to underestimate the deterministic component (Mello, 2009). Aiming at overcoming this issue and taking into account the steps proposed by Wangler and Backlund (2005) to study system behavior, we designed the hypothesis of this thesis ${ }^{3}$ as follows:

Time series influenced by additive noise can be decomposed into stochastic and

\footnotetext{
${ }^{1}$ The term deterministic means current observations only depend on past ones.

${ }^{2}$ The term stochastic means current observations may depend on past ones and most certainly on random variables.

${ }^{3}$ This thesis is based upon work supported by FAPESP (Sao Paulo Research Foundation), Brazil, under the grant 2009/18293-9.
} 
deterministic components in which individual models permit obtaining a hybrid one that improves accuracy.

In this hypothesis, the term additive noise means the time series observations are defined by the sum of the deterministic and the stochastic components. Hence, the objective of this research is to prove such hypothesis, which was performed in two steps. The first one comprises the process of decomposing a time series into two additive components: one stochastic and another deterministic. The second is related to the individual modeling of both components.

We started the proof of the first part of our hypothesis searching for methods to decompose time series. This process was performed considering a structured and documented search for published papers following the rules defined by the Systematic Literature Review (SLR) (Rios and Mello, 2012). Based on this review, we noticed spectral analysis methods are considered more appropriate to decompose time series. Although there exists several methods, we found a recent proposed one called Empirical Mode Decomposition (EMD) (Huang et al., 1998), which confirmed to be very useful to decompose time series regardless the system behavior used to produce their observations ${ }^{4}$.

In summary, the EMD method decomposes time series into a set of additive monocomponents called Intrinsic Mode Functions (IMFs). By analyzing IMFs, we notice the stochastic level tends to reduce as next monocomponents are extracted. Considering this observation, we developed a formal study, in which we proved, using the Nyquist-Shannon sampling theorem (Jerri, 1977), IMFs can be separated into two classes: one with highfrequency components and another containing low-frequency ones. According to the signal processing area, these classes correspond to stochastic and deterministic influences, respectively (Gonzalez and Woods, 2006).

After proving IMFs can be used to form the stochastic and deterministic components, we developed two approaches to decompose time series. The first one uses the Recurrence Plot (RP) method (Eckmann et al., 1987; Marwan et al., 2007) to analyze the determinism rate of IMFs extracted using EMD (Section 4.2). All IMFs with determinism rate greater than a threshold are added to form the deterministic component and the remaining ones are summed to form the stochastic component. As discussed along chapters, the main

\footnotetext{
${ }^{4}$ Chapter 2 presents more details on system behavior. To help the reader this essentially involves linearity, stationarity and stochasticity.
} 
limitation of this approach is the need for defining some parameters, which may influence the obtained stochastic and deterministic components. Although there are methods to estimate those parameters, this approach requires human supervision to define them.

This drawback motivated us to design the second approach (Section 4.3), which combines EMD and a continuous Mutual Information estimation method, proposed by Darbellay and Tichavský (2000), to obtain the stochastic and deterministic components in an unsupervised and nonparametric manner.

Experiments performed on synthetic datasets confirmed the importance of decomposing time series and individually modeling the deterministic and stochastic components, proving the second part of our hypothesis (see Chapter 5). Besides synthetic datasets, we also performed experiments on real-world datasets, whose results confirmed the importance of decomposing time series and evaluating the stochastic and deterministic influences, as stated by the first part of our hypothesis. In these experiments, we verified both components permit detecting patterns and extracting implicit information of time series. The analysis on real-world datasets was performed in collaboration with Prof. Lael Parrott and Prof. Holger Lange during a visit to the Université de Montréal - Canada. This visit was supported by CAPES (Coordination for Enhancement of Higher Education Personnel), which provided a scholarship for a doctoral internship in the form of Program Doctoral Sandwich Abroad (PDSE) under grant 5983-11-8. Currently, Prof. Parrott leads the Complex Environmental Systems Laboratory in the Department of Earth \& Environmental Sciences and Biology at the University of British Columbia (Canada) and Prof. Lange is with the Norwegian Forest and Landscape Institute (Norway).

The approaches designed to decompose time series also motivated the development of two measurements (Chapters 4 and 5). The first one, called Mean Distance from the Diagonal Line (MDDL), is used to evaluate forecasting methods. As main advantage this measurement evaluates predicted and expected observations considering not only the match among individual values but also the general behavior. The second measurement, called Determinism Rate, is interpreted as a method to evaluate the influence of the deterministic component on time series.

Besides the previous results, we collaborated with Prof. Michael Small to design two new methods to generate surrogate data (see Appendix C), which are not directly related 
to this work, but were also important to bring new ideas and provide background in this area. The main steps of this collaboration were conducted during a visit to the Department of Electronic and Information Engineering at the Hong Kong Polytechnic University (supported by FAPESP through the research contingency funds under grant 2009/18293-9). Recently, Prof. Small is with the School of Mathematics and Statistics at the University of Western Australia.

Surrogate data methods have been widely applied to produce synthetic data, while maintaining the same statistical properties as the original time series. The most commonly considered methods to generate surrogates were proposed by Theiler et al. (1992), which are based on the Fourier Transform, restricting their application to stationary time series. In this situation, the presence of trend produces spurious high frequencies, generating inconsistent surrogates. To address this issue, we present two new surrogate data methods based on EMD, which initially decompose time series into a set of monocomponents, allowing to separate the trend. Afterwards, Theiler's surrogate methods are applied on the detrended series producing surrogates. Using those methods, one can investigate the presence of linear and nonlinear Gaussian processes in time series, supporting nonstationary behavior.

This thesis is organized as follows:

- Chapter 2 shows an overview on time series analyses and the methods commonly considered to decompose time series;

- Chapter 3 presents a formal study on frequencies of Intrinsic Mode Functions, supporting the proof of the first part of the hypothesis;

- Chapter 4 describes the two new approaches to decompose time series into stochastic and deterministic components. Besides such approaches, this chapter still presents a measurement to evaluate the determinism rate of time series;

- Chapter 5 presents experimental analyses, which support the second part of our hypothesis. In addition, this chapter presents a new measurement to evaluate forecasting methods;

- Chapter 6 discusses results on real-world datasets. The results confirmed the importance of decomposing time series, detecting and extract patterns of time series; 
- Chapter 7 draws conclusions and discusses about future work;

- Appendix A details the Systematic Literature Review and the obtained results;

- Appendix B overviews Dynamical System concepts;

- Appendix C details the two methods designed to generate surrogate data. 
CHAPTER

2

\section{Time Series Decomposition}

\subsection{Introduction}

Time series is a sequence of observations collected over time and arranged in chronological order (Morettin and Toloi, 2004; Shumway and Stoffer, 2006; Chatfield, 2004; Kirchgässner and Wolters, 2007). An important characteristic of time series is the interval between observations. When observations are collected over every time instant in $\mathbb{R}_{+}$, the time series is referred to as continuous. On the other hand, a time series is called discrete when its observations are sampled at fixed and isolated time intervals. Examples of continuous and discrete time series are shown in Figure 2.1.

Another important characteristic of time series is the number of variables collected at every time instant, i.e., if a single variable is monitored, the time series has only one dimension and it is referred to as univariate. In contrast, if two or more variables are monitored at the same instant, the series is in a multidimensional space and it is called multivariate (Morettin and Toloi, 2004; Hamilton, 1994).

Besides these characteristics, time series can also be classified according to four application domains (Box et al., 1994): i) forecasting; ii) estimation of transfer functions; 


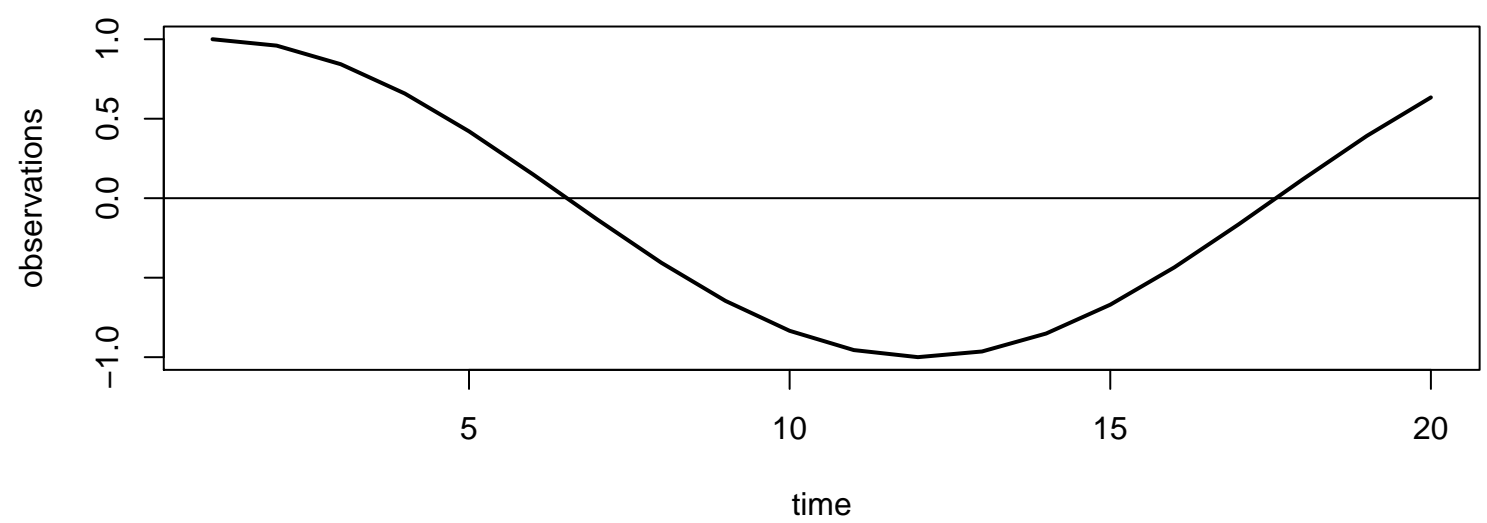

(a) Continuous time series

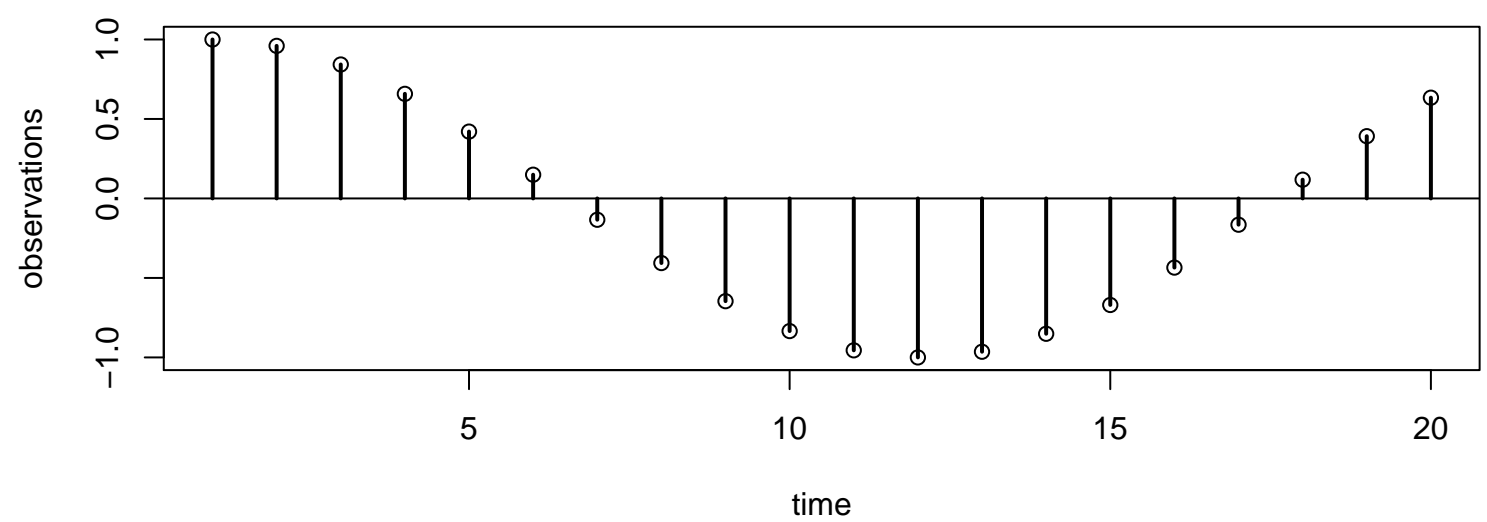

(b) Discrete time series

Figure 2.1: Examples of continuous and discrete time series. The discrete time series was sampled from the continuous one.

iii) analysis of effects of unusual intervention events on systems; and iv) discrete control systems.

The forecasting domain aims at determining a function that analyzes $n$ past observations of a time series $x(t)=\{x(n-1), \ldots, x(t-2), x(t-1), x(t)\}$ to estimate $l$ future observations $(\{x(t+1), x(t+2), \ldots, x(t+l)\})$, which are known as lead time. The main goal of this function is to reduce the difference between the estimated values $(\{\hat{x}(t+1), \hat{x}(t+2), \ldots, \hat{x}(t+l)\})$ and the expected ones $(\{x(t+1), x(t+2), \ldots, x(t+l)\})$. To find out this function, it is necessary to define the risks associated with every decision based upon the forecasts. These risks are expressed by calculating the upper and lower probability limits, as presented in Figure 2.2. By looking at this figure, we can notice that as more observations are predicted, the distance between the upper and lower limits increases. As a consequence, the accuracy of the forecasting function is reduced. 


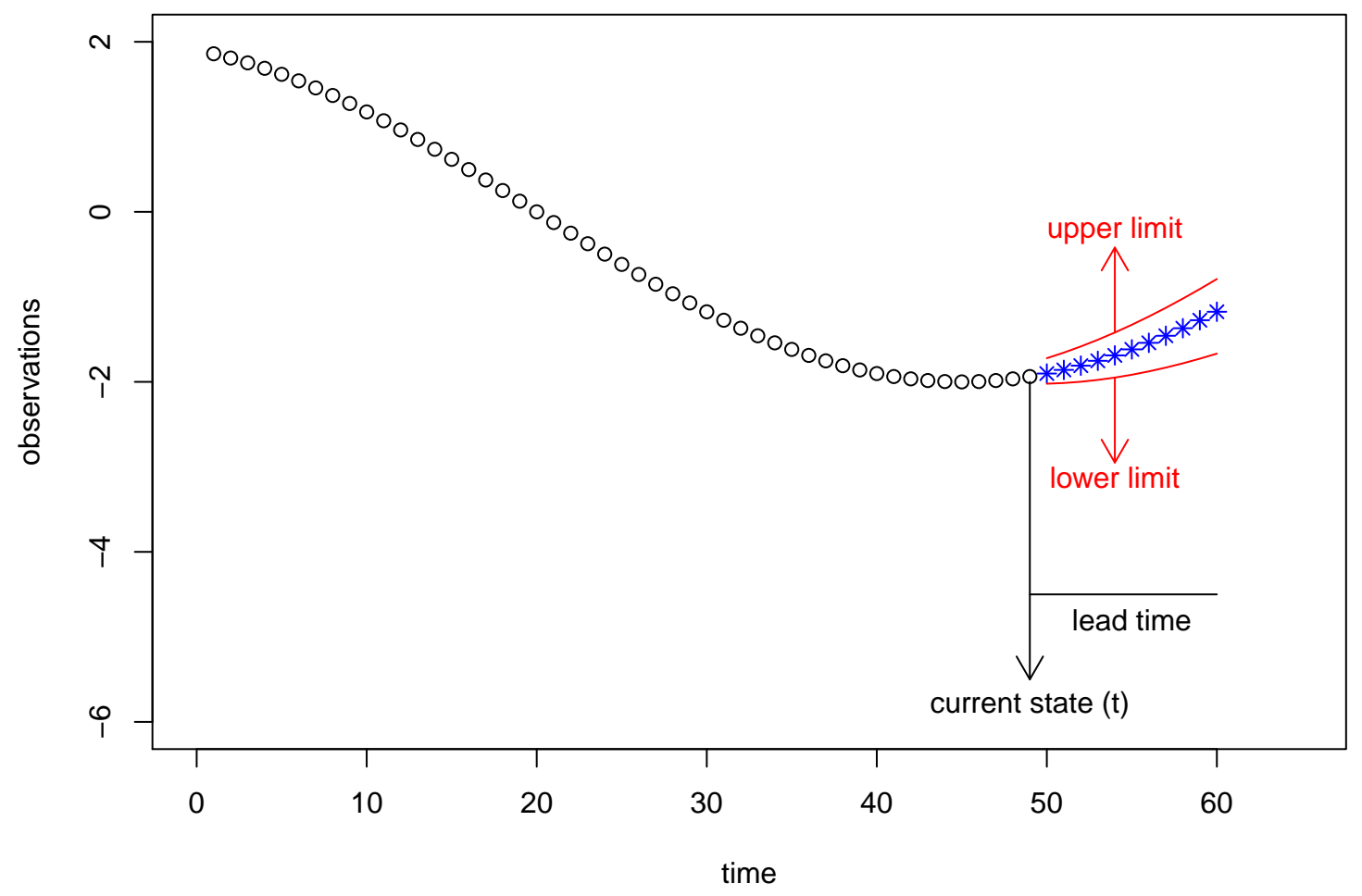

Figure 2.2: Example of a forecasting model: $\circ$ and $*$ represent the past and future observations, respectively (adapted from (Box et al., 1994)).

In the second domain, time series are used to estimate transfer functions, which permit understanding the behavior of systems. Instead of predicting, this application domain analyzes a set of previous observations to understand the current state of a system. The third domain uses time series to understand and measure the impact of a system on others as, for example, to correlate the effect of air pollution and the global warming. Finally, in the last domain, time series are used to monitor specific systems, aiming at detecting errors and taking decisions to adjust them to the expected behaviors.

The use of time series in those application domains depends on previous analyses, which are performed applying models adjusted on components embedded into observations. These components are shown in Equation 2.1, in which $x(t)$ represents a time series, $T(t)$ means the trend, $S(t)$ represents the seasonality, and $\varepsilon(t)$ represents a stochastic 
component.

$$
x(t)=T(t)+S(t)+\varepsilon(t)
$$

By knowing these components, one can assess the influence of important features that permit improving the accuracy of the estimated models. Among those features are stationarity, linearity, and stochasticity (Ishii et al., 2011).

A time series is said to be stationary when its observations are in a particular state of statistical equilibrium, in which its properties are not affected by a change of time origin, that is, the joint probability distribution remains the same by shifting any subset of observations forward or backwards over time (Box et al., 1994). Hence, if trend component $T(t)$ is present in a time series, it cannot be considered stationary, once its mean and variance change depending on the selected subset of observations, affecting the joint probability distribution.

On the other side, linear time series are those whose observations are composed of linear combinations of past occurrences and noises. Finally, stochastic time series are composed of random observations and relations, which follow probability density functions and may change over time. In contrast, deterministic time series strictly depend on past observations produced by some mathematical function or map (Box et al., 1994; Alligood et al., 1997).

Although it is very important to consider all the previous features when modeling a time series, in this work, we are interested in analyzing the determinism and stochasticity present in discrete and univariate time series.

In this sense, when a series is classified as deterministic, models from the Dynamical Systems area, specifically those from the Chaos Theory (Alligood et al., 1997), provide better results. When a series presents stochastic behavior, statistical models, such as the ones proposed by Box et al. (1994), are more appropriate. However, time series collected from real-world systems usually present a mixture of both components, i.e., the value of a single observation is influenced by deterministic and stochastic components. In such situation, the discard of any component may depreciate the quality of the resultant model (Han and Liu, 2009), i.e., the application of Dynamical System techniques on such time series tends 
to generate malformed attractors ${ }^{1}$, whereas statistical techniques tend to underestimate the deterministic part of the system.

Aiming at overcoming this issue, Wangler and Backlund (2005) proposed a methodology to study system behaviors in three well-defined steps: i) firstly, the system is decomposed in parts or components; ii) secondly, models are used to represent the behavior of each component; iii) at last, the models are combined to describe the global system behavior. However, the decomposition of time series is not a simple task, once we do not know the influence of every component on observations.

In this sense, many researchers have been proposing new techniques to decompose time series, what motivated the execution of a structured and documented search for published papers following the rules defined by the Systematic Literature Review (SLR) method (Rios and Mello, 2012).

According to the results obtained from the $\mathrm{SLR}^{2}$, we conclude spectral analysis methods are the most commonly considered to decompose time series (Rios and Mello, 2012). In the following sections, we present the spectral analysis methods considered more appropriate to decompose time series into stochastic and deterministic components.

\subsection{Decomposition methods}

A set of spectral-analysis techniques are commonly used to decompose time series, such as Principal Component Analysis (Jolliffe, 2002), Singular Spectrum Analysis (Golyandina et al., 2001), Fourier Transform (Graps, 1995), Wavelets (Graps, 1995), and Empirical Mode Decomposition (Huang et al., 1998).

Principal Component Analysis (PCA) is a nonparametric technique to reduce data dimensionality, modifying data points according to eigenvectors or extract relevant information from data (Jolliffe, 2002). Despite the applicability of this technique, PCA is not used in this work since it is limited to linear data.

Singular Spectrum Analysis (SSA) is a data-adaptive technique to decompose time series into orthogonal modes (Golyandina et al., 2001) and has been widely adopted due to its ability to identify and extract oscillatory components. By using this technique, one can de-

\footnotetext{
${ }^{1}$ More details about attractors and Dynamical System techniques are presented in Appendix B

${ }^{2}$ Details about the Systematic Literature Review can be found in Appendix A
} 
compose time series into three basic components: noise, trend, and seasonality. Although this technique has proven to be very successful in the analysis of climatic, meteorological and geophysical time series, we do not use it in this work due to its linear-oriented approach: SSA is essentially an application of PCA in the time domain (Hsieh and Wu, 2002; Schoellhamer, 2001). A nonlinear version of SSA, combining time-domain PCA with artificial neural networks, is presented in (Hsieh and Wu, 2002); however, this is far from being an established extension and is not considered in this work.

Fourier Transform (FT) is the classical technique to represent data using sums of sinusoidal signals (Huang et al., 1998). Its ability to describe time series components makes it widely applicable to different real-world problems, including the decomposition of time series (Graps, 1995). However, FT is not applicable in the context of this work because it assumes series are linear and strictly periodic and stationary (Huang et al., 1998).

Wavelet Transform (WT) is an alternative to PCA and FT. Wavelets are written as mathematical functions to decompose time series at different scales and resolutions (Graps, 1995). This decomposition makes it possible to analyze time series not only in the frequency domain, like FT, but also locally in the time domain. Consequently, WT keeps temporal relations and features among time series observations, allowing its application on stationary data. Similarly to FT, WT was not adopted in this work because it is also a linear-oriented technique (Huang et al., 1998).

In this work, we considered an alternative method called Empirical Mode Decomposition (EMD) (Huang et al., 1998), which supports the decomposition of time series regardless linearity, stationarity and stochasticity. This method converts a time series into a set of monocomponents, called Intrinsic Mode Functions (IMFs), to support the study of instantaneous frequencies and amplitudes using the Hilbert Spectral Analysis (HSA) (Huang et al., 1998).

The key step in performing this decomposition is the sifting process (Figure 2.3), which initially analyzes a time series $x(t)$ and identifies local maxima and minima observation values along time (Figure 2.3(a)). Afterwards, all maxima are combined to compose an upper envelope $l(t)$ by using the cubic spline method (Figure 2.3(b)). The same procedure is applied to all minima to compose a lower envelope $u(t)$ (Figure 2.3(c)) (Huang et al., 1998). Next, the approximation values obtained using both cubic splines (lower and upper) 
are used to compute the mean envelope (Equation 2.2) as illustrated in Figure 2.3(d).

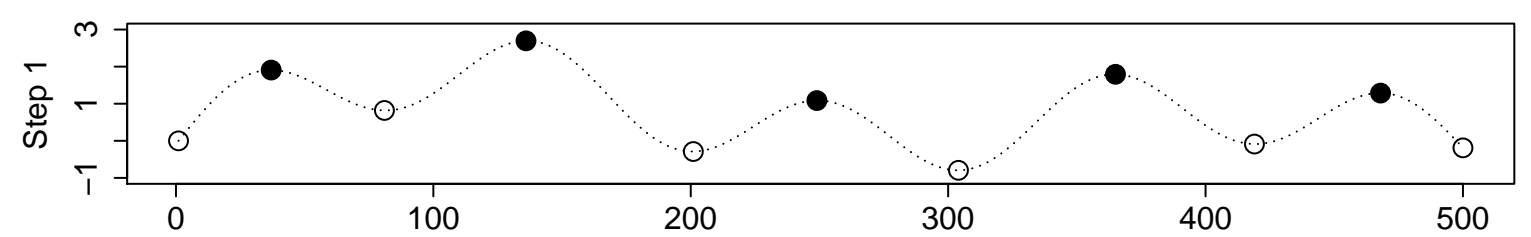

(a)

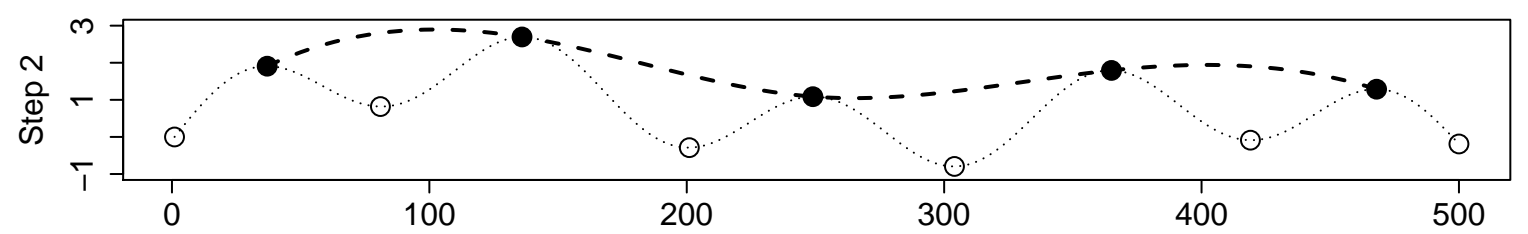

(b)

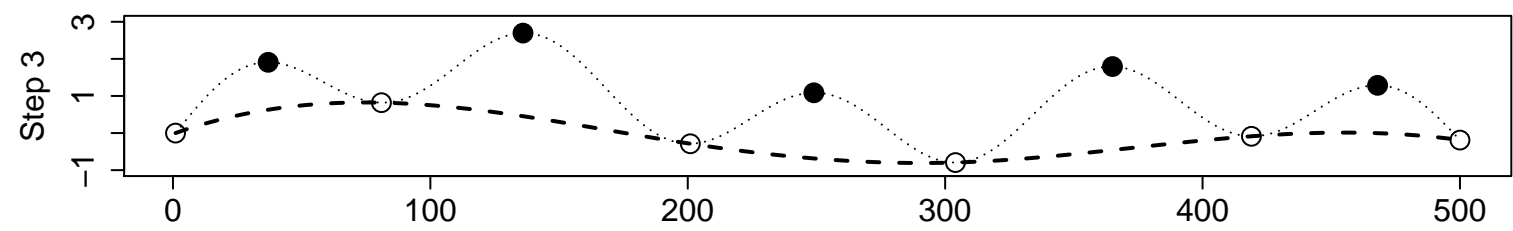

(c)

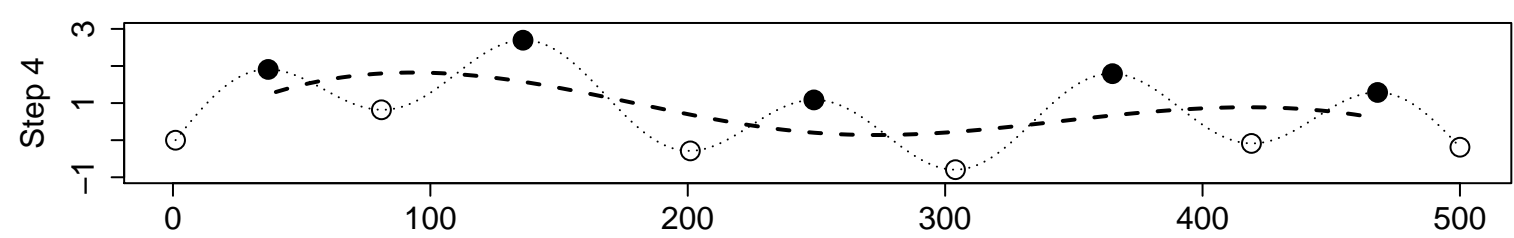

(d)

Figure 2.3: The original time series is represented by the dotted line in all plots. (a) The maxima and minima observations are selected. $(b-c)$ The cubic spline method is applied to the maxima and the minima, connecting all observations and producing the upper and lower envelopes, respectivelly. (d) The mean envelope is obtained by calculating the mean values between the upper and lower envelopes.

$$
m(t)=\frac{l(t)+u(t)}{2}
$$

Later, $m(t)$ is removed from the original time series $x(t)$, producing the first monocomponent candidate $h_{1,1}(t)=x(t)-m(t)$, in which the first index corresponds to the IMF identifier (as this is the first IMF to be extracted, the index is one) and the second refers to the candidate identifier (as this is the first candidate, the index is also one). This first candidate $h_{1,1}(t)$ is used in place of the original data and the sifting process is repeated until the candidate satisfies the IMF monocomponent definition, which must agree with one of the following requirements: i) the number of extrema and the number of zero-crossings must 
be either equal or differ at most by one; or ii) for every $t, m(t)$ is zero. After obtaining the first monocomponent candidate that satisfies the IMF definition, the first IMF monocomponent is obtained according to $h_{1}(t)=h_{1, k}(t)$, assuming $k$ candidates were produced until reaching the IMF definition.

This first IMF is then removed from data, $x(t)-h_{1}(t)$, and the resultant series is again analyzed by the whole process, producing further IMFs until reaching the stop criterion, i.e., when the last IMF becomes a monotonic function, from which no further IMF can be extracted from. Hence, this last component is referred to as final residue $r(t)$ (Huang et al., 1998). In summary, according to EMD, a time series $x(t)$ is composed of a set of IMF monocomponents plus a residue as presented in Equation 2.3.

$$
x(t)=\sum_{n=1}^{N} h_{n}(t)+r(t)
$$

In order to better understand the IMFs produced by EMD, consider a time series $x(t)$ with additive noise created from the combination of two different components: one deterministic and another stochastic. In this example, the deterministic component was created using a sine function with angular frequency equals to $2 \pi$. The stochastic component was produced by a random process $\varepsilon(\mu, \sigma)$ following a Normal distribution with mean $\mu=0$ and standard deviation $\sigma=1$. Figure 2.4 presents these two components.

Hence, the noisy time series was obtained by summing both components, $x(t)=$ $\sin (2 \pi t)+\varepsilon(0,1)$. This series was analyzed using the EMD method which produced a set of IMFs $h_{n}(t)$ plus a residue $r(t)$ as presented in Figure 2.5.

In this thesis, we adopted EMD to decompose time series due to the possibility of analyzing a wider class of time series, once its applicability is not limited to linear and stationary data. Besides this, we empirically observed the obtained IMFs present different levels of stochasticity (see Figure 2.5). This motivated us to develop a formal study, presented in the next chapter, proving the first part of our hypothesis, which states time series can be decomposed into stochastic and deterministic components. 


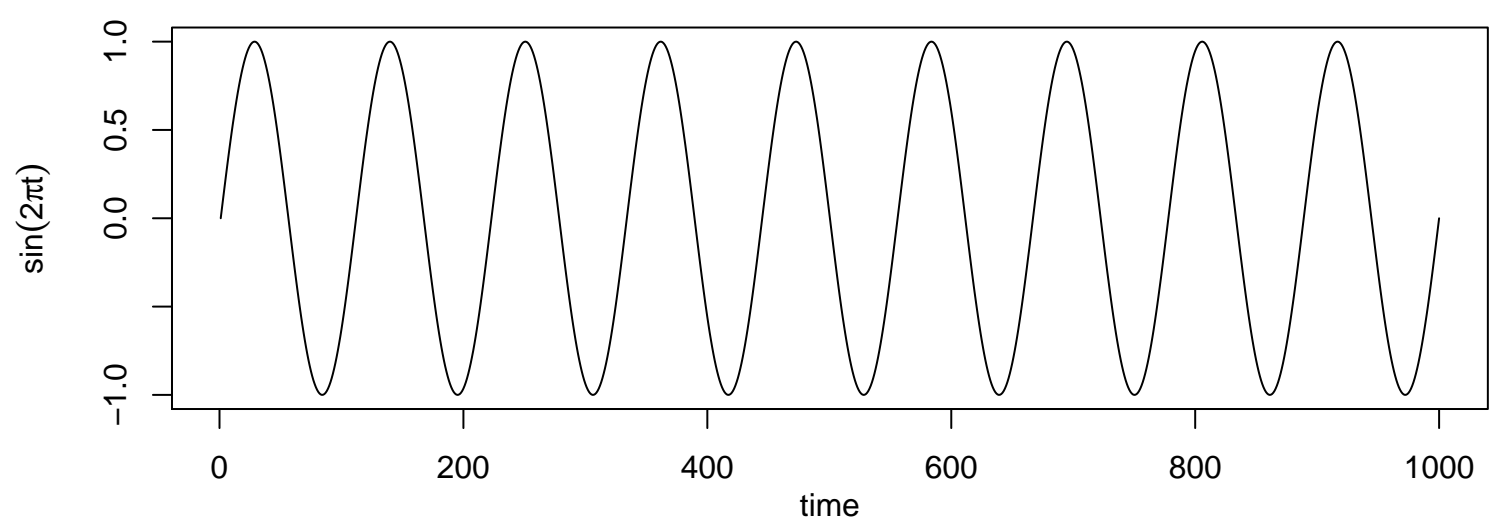

(a)

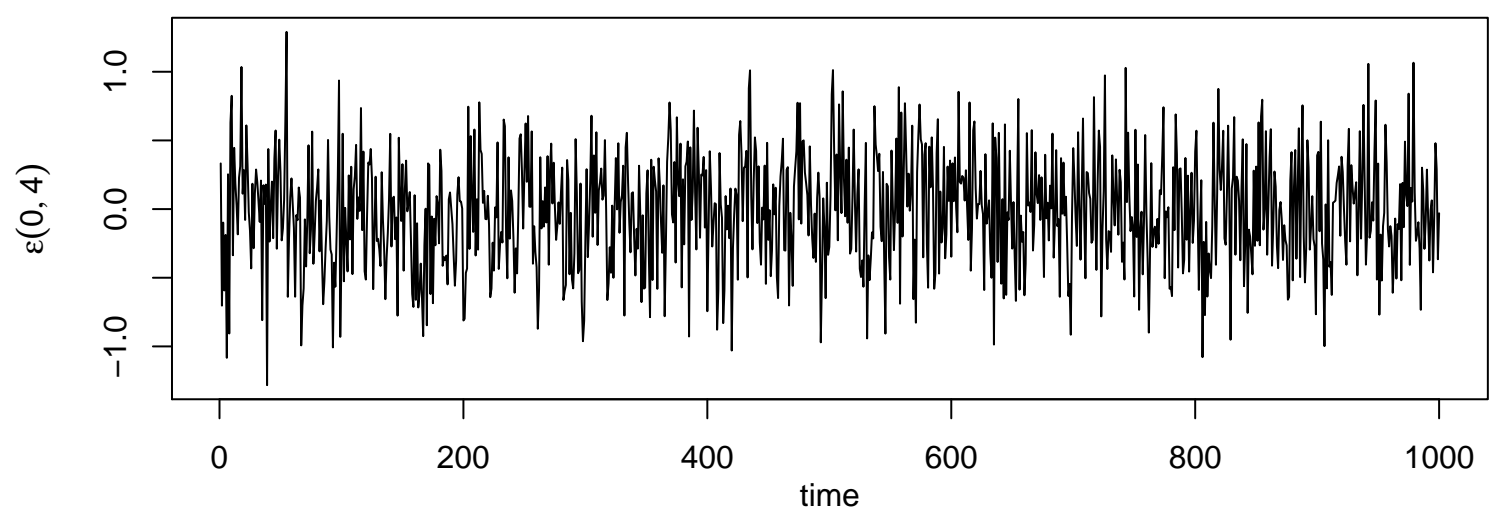

(b)

Figure 2.4: The (a) deterministic, $\sin (2 \pi t)$, and (b) stochastic, $\varepsilon(0,4)$, components used to create the noisy time series $x(t)$.

\subsection{Concluding remarks}

Despite the Empirical Mode Decomposition method has been widely adopted to decompose time series (de Lima et al., 2006; Huang et al., 2001; Kopsinis and McLaughlin, 2009; Lee and Ouarda, 2012; Meyer and Stiedl, 2006; Wu and Huang, 2004), Colominas and Gastón Scholotthauer (2012) pointed out a problem when the same IMF has very different amplitudes along time or different IMFs have similar oscillations in amplitudes, what is named as mode mixing. An example of the mode mixing problem is observed in IMFs 5 and 6 presented in Figure 2.5. In IMF 5, amplitude is lower in range $[400,800]$ than the remaining of the time series, whereas IMF 6 presents an opposite behavior. By looking at these IMFs we observe part of IMF 5 is embedded into IMF 6 and vice versa. 

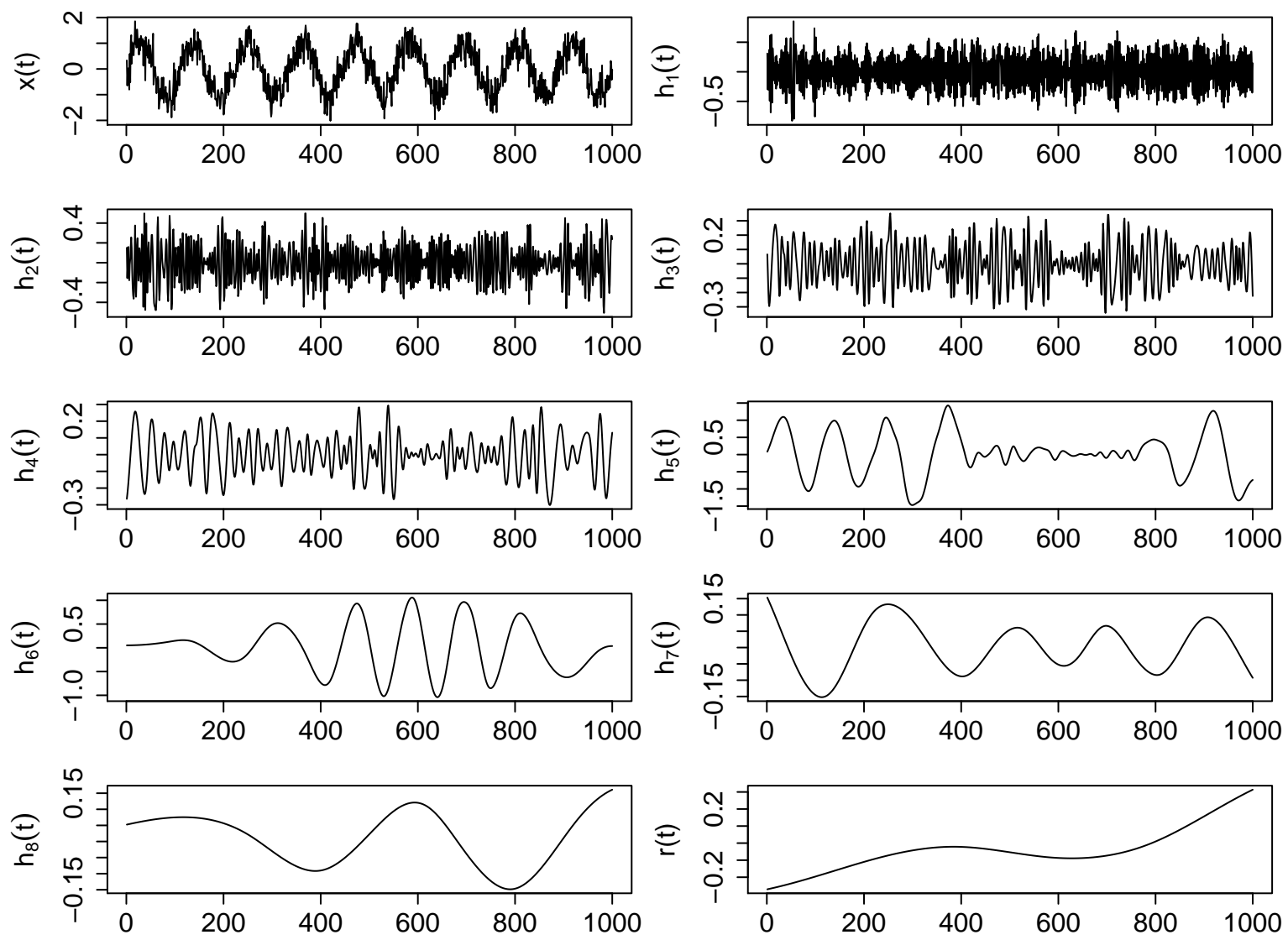

Figure 2.5: IMFs $\left(h_{n}(t)\right)$ and residue $(r(t))$ extracted from a noisy time series $x(t)=\sin (2 \pi t)+$ $\varepsilon(0,1)$. The $x$-axis represents the time in all plots.

Aiming at solving this problem, Colominas and Gastón Scholotthauer (2012) developed a new method called Ensemble Empirical Mode Decomposition (EEMD). However, this method was not evaluated in this thesis because the mode mixing problem does not affect our study. As presented in the following chapters, we are interested in decomposing time series into two components (one stochastic and another deterministic), what suppresses the mode mixing problem as depicted later. 


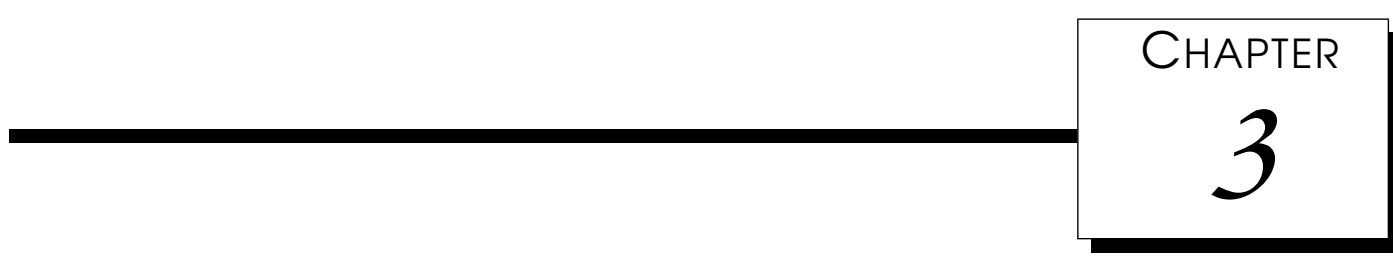

\section{Formal Definition On Time Series}

\section{Decomposition}

\subsection{Introduction}

The main goal of this thesis is to prove the hypothesis presented in Chapter 1, in which we state time series can be separated into stochastic and deterministic components in order to model its observations at a higher accuracy. Hence, the first step to prove this hypothesis is to find out an approach that permits understanding the influences of the stochastic and deterministic components separately.

Among all methods proposed to decompose time series (see Chapter 2), Empirical Mode Decomposition (EMD) was adopted because it extracts a finite set of Intrinsic Mode Functions (IMFs), which contains important individual characteristics of series regardless linearity, stationarity, and stochasticity (Huang et al., 1998).

By studying EMD, we observed this method produces IMFs at different frequency bandwidths. This observation also called the attention of other researchers such as Flan- 
drin et al. (2004), which, using numerical experiments, demonstrated EMD essentially acts as a dyadic filter bank. Based on this conclusion, we developed a new proof using the Nyquist-Shannon sampling theorem (Jerri, 1977) confirming the separation of IMFs into two classes: one with higher-frequency components and another containing lowerfrequency ones. As typically considered in the signal processing area, the first class corresponds to stochastic influences, whereas the second represents deterministic ones. In the following sections, we present all steps of this proof in details.

\subsection{On the Nyquist-Shannon sampling theorem}

According to the Nyquist-Shannon sampling theorem (Jerri, 1977), a band-limited time series $x(t)$ can be correctly reconstructed from an infinite sequence of samples. A time series is considered band-limited when its frequencies are restricted to a bandwidth of $B$ hertz, determined by the difference between the highest $\left(f_{h}\right)$ and lowest $\left(f_{l}\right)$ frequencies, i.e., $B=f_{h}-f_{l}$.

In order to reconstruct a time series $x(t)$, the sampling frequency $f_{s}$ should be greater than twice the highest frequency, $f_{s}>2 f_{h}$ (Jerri, 1977). By assuming the lowest frequency is equal to zero, the bandwidth can be rewritten as $B=f_{h}$ and the sampling frequency is commonly defined as $f_{s}>2 B$. The Nyquist frequency $B$ and rate $f_{s}$ are defined as $f_{s} / 2$ and $2 B$, respectively, therefore, a band-limited time series can be correctly reconstructed when the sampling rate has exceeded $f_{s}$.

In order to formally describe the Nyquist-Shannon sampling theorem, consider the Fourier transform $X(f)$ for a continuous time series $x(t)$, as defined in Equation 3.1.

$$
X(f) \stackrel{\text { def }}{=} \frac{1}{2 \pi} \int_{-2 \pi W}^{2 \pi W} x(t) e^{-i f t} \mathrm{~d} t
$$

The time series $x(t)$ is said to be band-limited when its Fourier coefficients $X(f)$ are zero outside the band interval $I=[-2 \pi W, 2 \pi W]$, i.e., if $X(f)=0, \quad \forall f \notin I$. According to the Nyquist-Shannon sampling theorem, a time series $x(t)$ is sufficiently reconstructed if time-spaced observations are collected at regular and constant time intervals, as defined in Equation 3.2, in which $f$ is the maximum represented frequency when observations are 
collected at $T$ time intervals.

$$
T \stackrel{\text { def }}{=} \frac{1}{f}
$$

When interpolating any time series $x(t)$, a subset of observations $D(x(t))$ of $x(t)$ is selected. Assuming $x(t)$ is continuous, and therefore, has an infinite number of observations, $D(x(t))$ has a finite number of observations, which is used to construct new data points within the time range of $x(t)$. As a corollary of the Nyquist-Shannon sampling theorem, by increasing the time interval between consecutive observations, the interpolation of $D(x(t))$ will represent lower frequencies than those of $x(t)$.

\subsection{Analyzing frequencies of Intrinsic Mode Functions}

The first proof is started by remembering a next IMF produced by EMD contains lower frequencies than previous ones (Section 2.2). To clarify it, consider a time series $x(t)$ composed of $j$ IMFs, $\left\{h_{1}(t), h_{2}(t), \cdots, h_{j}(t)\right\}$, which were extracted using EMD. Let $\mathcal{F}_{\max }(\cdot)$ be a function to compute the maximum frequency. We can define the following hypothesis: $\mathcal{F}_{\max }\left(h_{1}(t)\right)>\mathcal{F}_{\max }\left(h_{2}(t)\right)>\cdots>\mathcal{F}_{\max }\left(h_{j-1}(t)\right)>\mathcal{F}_{\max }\left(h_{j}(t)\right)$, i.e., the maximum frequency of a next IMF is always lower than the previous one.

By proving this hypothesis, we can demonstrate that EMD sifting process acts as a filter, removing components according to their frequencies. Therefore, the deterministic influence increases as the frequency is reduced. In order to prove this hypothesis, we first start defining the following theorem:

Theorem: If the maximum frequency of a next Intrinsic Mode Function produced by EMD is lower than the previous one, $\mathcal{F}_{\max }\left(h_{j-1}(t)\right)>\mathcal{F}_{\max }\left(h_{j}(t)\right)$, then EMD works as a frequency filter and, consequently, supports the separation of deterministic and stochastic influences.

Proof: Considering the sifting process (presented in Section 2.2), the first EMD step is performed by calculating the time series envelopes according to the following definitions.

Definition 1 (Envelopes). First, the time series extrema are selected. Then, all maxima are connected by using a cubic spline function, producing the upper envelope $u(t)$ 
(Equation 3.3). The same occurs with all minima connected to form the lower envelope $l(t)$ (Equation 3.4).

$$
\begin{aligned}
& u(t)=a^{\prime} x(t)^{3}+b^{\prime} x(t)^{2}+c^{\prime} x(t)+d^{\prime} \\
& l(t)=a^{\prime \prime} x(t)^{3}+b^{\prime \prime} x(t)^{2}+c^{\prime \prime} x(t)+d^{\prime \prime}
\end{aligned}
$$

Next, the sifting process estimates the mean envelope, as shown in the following definition.

Definition 2 (Mean envelope). The mean envelope is calculated by Equation 3.5.

$$
\begin{aligned}
& m(t)=\frac{u(t)+l(t)}{2} \\
& m(t)=\frac{\left[a^{\prime} x(t)^{3}+b^{\prime} x(t)^{2}+c^{\prime} x(t)+d^{\prime}\right]+\left[a^{\prime \prime} x(t)^{3}+b^{\prime \prime} x(t)^{2}+c^{\prime \prime} x(t)+d^{\prime \prime}\right]}{2} \\
& m(t)=\frac{\left(a^{\prime}+a^{\prime \prime}\right)}{2} x(t)^{3}+\frac{\left(b^{\prime}+b^{\prime \prime}\right)}{2} x(t)^{2}+\frac{\left(c^{\prime}+c^{\prime \prime}\right)}{2} x(t)+\frac{\left(d^{\prime}+d^{\prime \prime}\right)}{2}
\end{aligned}
$$

Remark 1. According to Equation 3.5, the mean envelope is still considered a cubic spline function, once its properties and characteristics have been preserved.

After calculating the mean envelope, the next step is to remove it from time series $x(t)$, producing an IMF candidate $h_{1,1}(t)$ (the first index represents the IMF identifier and the second corresponds to the candidate identifier). This IMF candidate is used in place of $x(t)$ and all previous steps are then repeated (Equation 3.6) until reaching a stop criterion, as shown in Section 2.2 . 


$$
\begin{aligned}
h_{1,1}(t) & =x(t)-m_{1}(t) \\
h_{1,2}(t) & =h_{1,1}-m_{2}(t) \\
h_{1,3}(t) & =h_{1,2}-m_{3}(t) \\
& \cdots \\
h_{1, k}(t) & =h_{1, k-1}-m_{k}(t)
\end{aligned}
$$

Therefore, considering at least one of the IMF requirements was met after $k$ iterations (see Section 2.2), the first real IMF is obtained by Equation 3.7.

$$
h_{1}(t)=h_{1, k}(t)
$$

Definition 3 (Intrinsic Mode Function). IMF is defined in terms of intermediary mean envelopes as shown in Equation 3.8. In such equation, we only formalized how the first IMF $h_{1}(t)$ was obtained, but the remaining ones are produced by using the same steps (the $q$-th IMF, i.e., $h_{q}(t)$, is obtained by replacing the original time series $x(t)$ by $x(t)-\sum_{i=1}^{q-1} h_{i}(t)$, as discussed in Section 2.2).

$$
\begin{aligned}
& h_{1}(t)=x(t)-m_{1}(t)-m_{2}(t)-m_{3}(t)-\cdots-m_{k}(t) \\
& h_{1}(t)=x(t)-\sum_{i=1}^{k} m_{i}(t)
\end{aligned}
$$

The next step is the analysis of the definition resultant from the Nyquist-Shannon sampling theorem, shown in Equation 3.2. This definition is used to establish a relationship between the number of observations selected to compose the extrema and the time series frequency. By selecting observations to represent the minima and maxima values (Figure 2.3), only a subset of the original time series are used to construct the mean envelope, thus, the average time interval $\triangle T$ between consecutive observations increases in relation to the original time series, reducing the maximum frequency. This behavior is reflected on the mean envelope, whose maximum frequency is lower than that of the original time 
series.

In order to prove this assumption, consider $\Delta T_{x(t)}$ and $\triangle T_{m_{1}(t)}$ the time intervals for the original time series $x(t)$ and the first mean envelope $m_{1}(t)$, which is an interpolation based on a subset of the original observations in $x(t)$. The relation between these intervals is expressed in Equation 3.9.

$$
\triangle T_{x(t)}<\triangle T_{m_{1}(t)}
$$

Considering the Nyquist-Shannon definition, in which the time interval $\triangle T$ and frequency $f$ in the time series are inversely proportional, we extended Equation 3.9 into Equation 3.10.

$$
\begin{aligned}
\frac{1}{f_{x(t)}}<\frac{1}{f_{m_{1}(t)}}, \text { thus } \\
f_{x(t)}>f_{m_{1}(t)}
\end{aligned}
$$

Therefore, the maximum frequency of the first mean envelope $\left(m_{1}(t)\right.$ in Equation 3.6) is lower than that of the original time series $x(t)$. In the next sifting step, the time series is replaced by the first IMF candidate, $h_{1,1}(t)=x(t)-m_{1}(t)$, and the frequencies of the next mean envelope, $m_{2}(t)$, will be even lower than $m_{1}(t)$. Thus, the frequency of the first IMF $\left(h_{1}(t)\right.$, in Equation 3.8) will be higher than $m_{1}(t)$ and higher than every mean envelope $m_{i}(t), \forall i=1, \ldots, k$. The next step in the EMD process is performed by removing the first IMF from the time series, i.e., $x(t)-h_{1}(t)$, and the resultant time series is again analyzed by the whole process, producing further IMFs until reaching the stop criterion. Therefore, the maximum frequency of the next IMF will be lower than the previous one. As result, we state EMD works as a frequency filter, removing components from high to low frequencies.

We conclude that a next IMF produced by EMD always contains less stochastic and more deterministic influence, what allows to create two individual components: one stochastic and another deterministic as necessary to prove the stated hypothesis. 


\subsection{Simulation results}

This section presents an experimental analysis to illustrate the proof discussed in the previous section. The experiment was performed by analyzing a time series composed of two distinct components: one deterministic and another stochastic. The deterministic component was created considering a sine function with angular frequency $2 \pi$. The stochastic component was produced by a random process $\varepsilon(\mu, \sigma)$ following a Normal distribution with mean $\mu=0$ and standard deviation $\sigma=1$. The time series used in our experiment was obtained by summing both components $x(t)=\sin (2 \pi t)+\varepsilon(0,1)$.

Initially, $x(t)$ was decomposed by the EMD method, producing a set of IMFs $h_{j}(t)$ plus a residue, as shown in Figure 3.1.

Then, we analyzed each IMF by calculating their periodograms (Shumway and Stoffer, 2006) according to Equation 3.11, in which $X\left(h_{q}(t)\right)$ corresponds to the Fourier coefficients for the $q$-th IMF (Equation 3.1).

$$
\mathcal{I}\left(h_{q}(t)\right)=\left|X\left(h_{q}(t)\right)\right|^{2}
$$

In order to illustrate the previously presented proof, we must compare the difference in terms of the maximum frequencies between consecutive IMFs. This comparison is performed analyzing how the density of frequencies changes between consecutive IMFs using the weighted mean frequency $\overline{\mathcal{F}}(\cdot)$ expressed in Equation 3.12, which considers the frequency $(f, \forall f \in\{1,2, \cdots, t / 2\})$ and spectrum $(\mathcal{I}(\cdot))$ values obtained using the periodogram.

$$
\overline{\mathcal{F}}\left(h_{q}(t)\right)=\frac{\sum_{n=1}^{t} f \cdot \mathcal{I}\left(h_{q}(n)\right)}{1+\sum_{n=1}^{t} \mathcal{I}\left(h_{q}(n)\right)}
$$

Figure 3.2 shows the periodogram for each IMF and the weighted mean frequencies $\overline{\mathcal{F}}(\cdot)$, whose individual values are shown in Table 3.1.

Figure 3.2(a) shows the weighted mean frequency for the first IMF is located in range $[300,400]$. Figure 3.2(b) shows the maximum frequency for the second IMF is located below value 200 . The next plots show weighted mean frequencies are reduced as further IMFs are extracted. These results indicate the maximum frequencies are reduced in next IMFs (at 


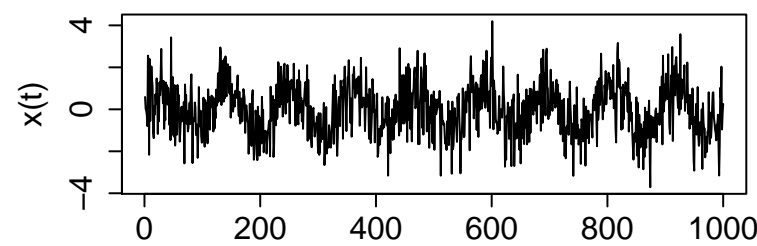

(a)

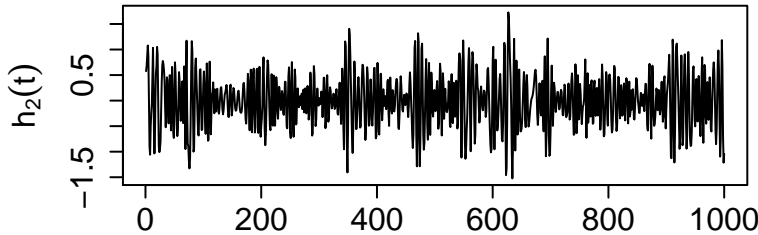

(c)

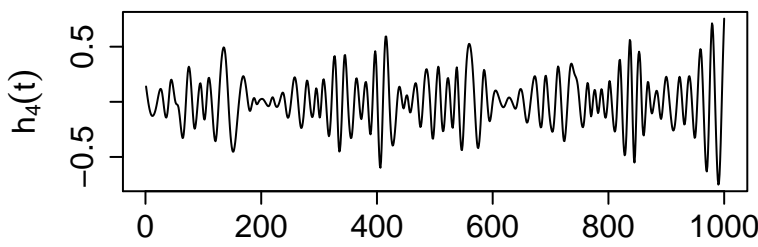

(e)

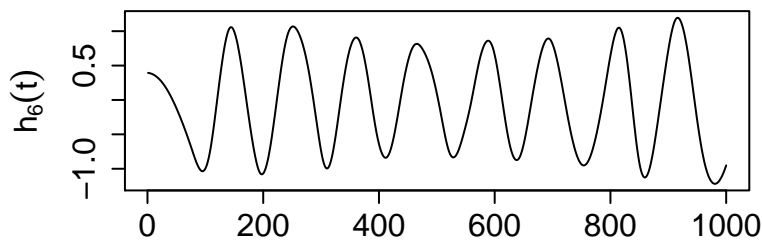

(g)

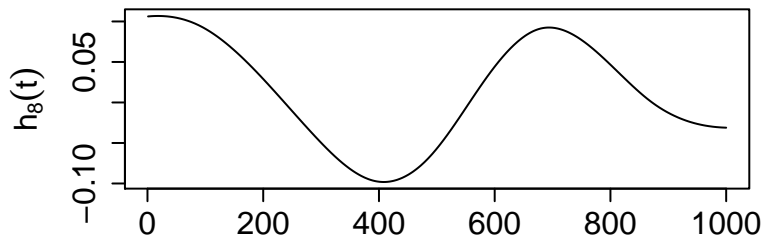

(i)

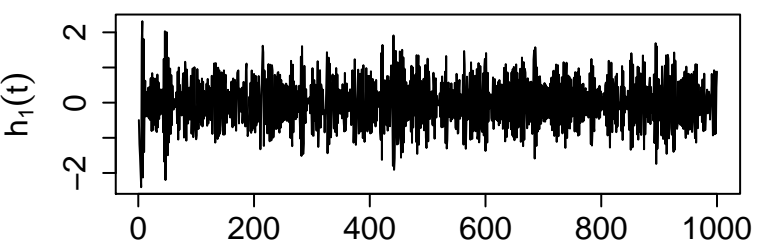

(b)

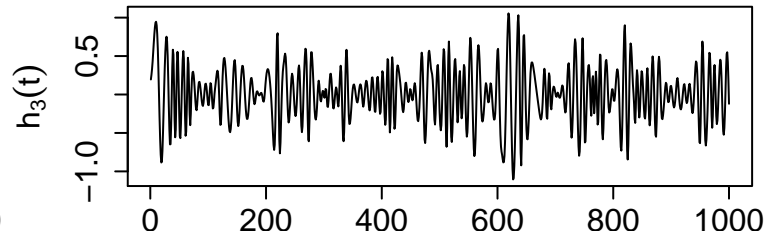

(d)

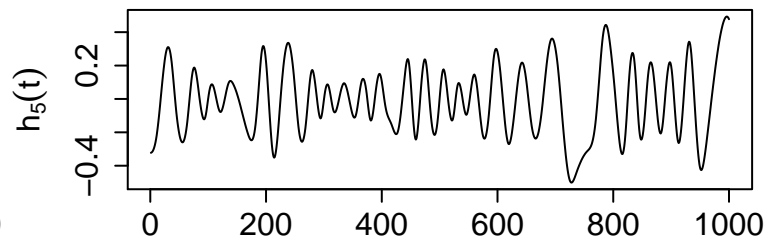

(f)

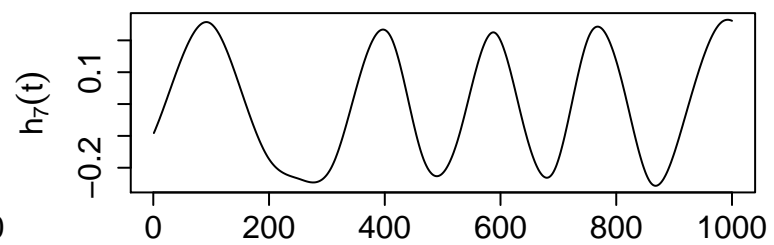

(h)

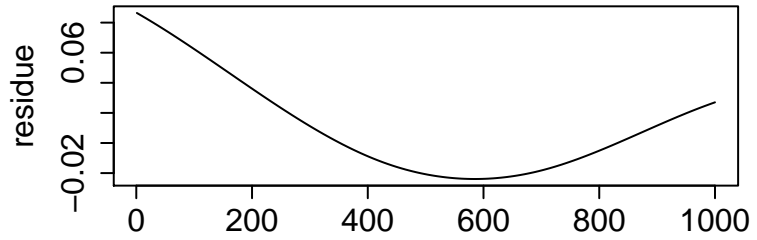

(j)

Figure 3.1: The noisy time series $x(t)=\sin (2 \pi t)+\varepsilon(0,1)$ is shown in plot (a). Plots from (b) to (i) show all IMFs $h_{n}(t)$ extracted at each iteration from the time series $x(t)$ using the EMD method. The last plot (j) shows the residue $r(t)$. The $x$-axis represents the time in all plots.

higher indices), illustrating the theorem proved in the previous section. 


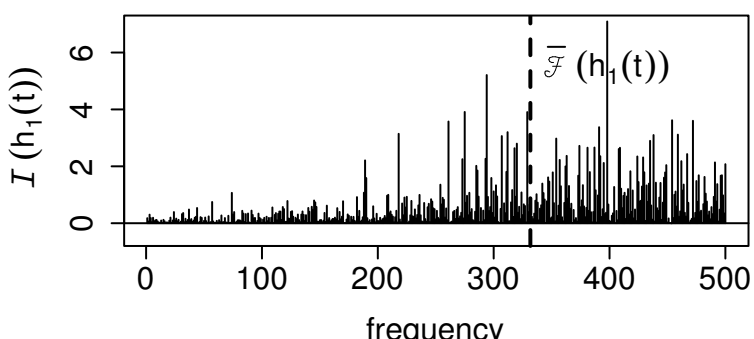

(a)

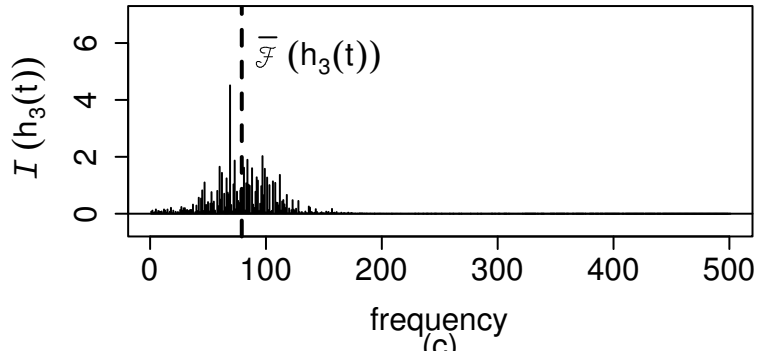

(c)

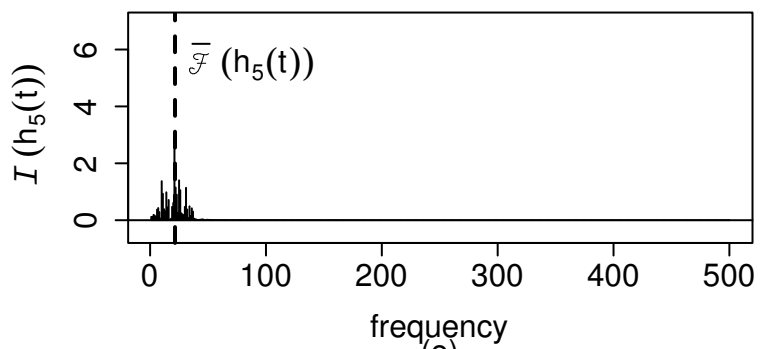

(e)

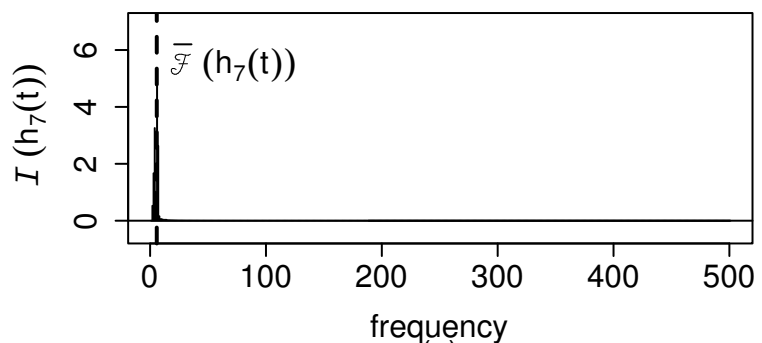

(g)

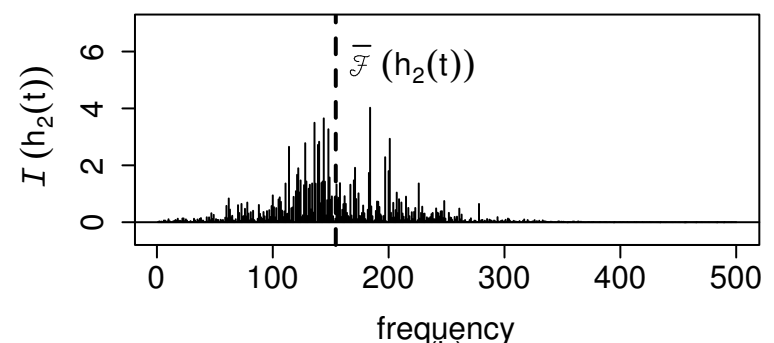

(b)

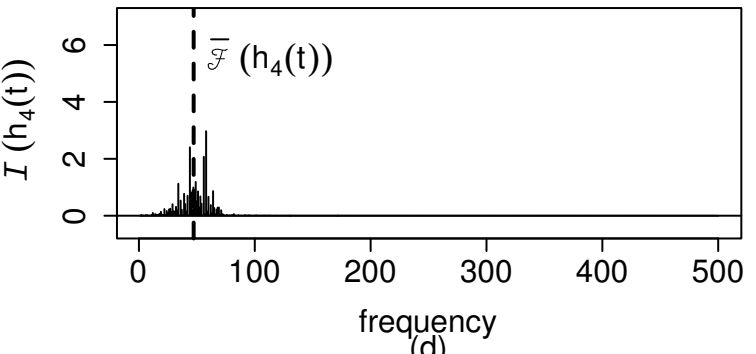

(d)

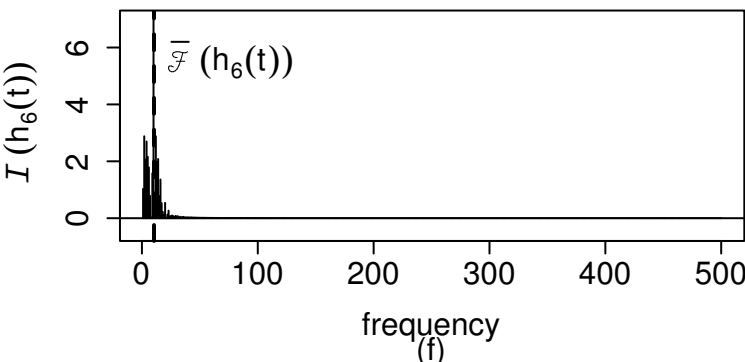

(f)

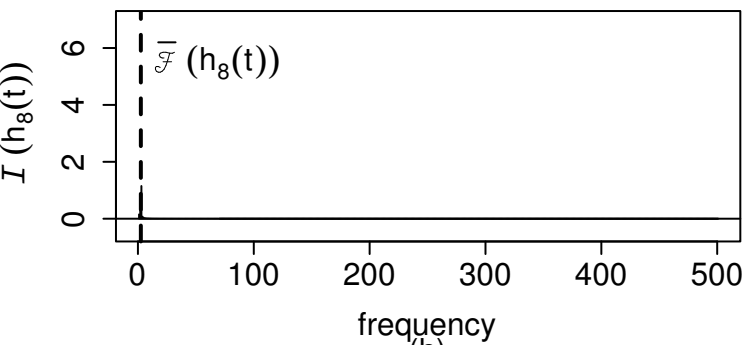

(h)

Figure 3.2: Periodogram for each extracted IMF. The vertical dotted line represents the mean weighted frequency $\overline{\mathcal{F}}(\cdot)$ defined in Equation 3.12. By considering these plots, we notice the maximum frequency is reduced as new IMFs are iteratively extracted.

\subsection{Concluding remarks}

The study on frequencies of IMFs extracted by Empirical Mode Decomposition has been approached by researchers such as Flandrin et al. (2004), who demonstrated EMD essentially acts as a dyadic filter bank resembling Wavelet decomposition. In this section, we extended the conclusion drawn by Flandrin et al. (2004), presenting a proof that EMD 
Table 3.1: Weighted mean frequencies for all IMFs illustrated in Figure 3.1.

\begin{tabular}{c|c}
\hline IMFs & $\overline{\mathcal{F}}\left(h_{q}(t)\right)$ \\
\hline \hline$h_{1}$ & 331.67 \\
$h_{2}$ & 154.37 \\
$h_{3}$ & 79.11 \\
$h_{4}$ & 47.24 \\
$h_{5}$ & 21.48 \\
$h_{6}$ & 10.43 \\
$h_{7}$ & 5.74 \\
$h_{8}$ & 2.51 \\
\hline \hline
\end{tabular}

permits separating the stochastic from the deterministic influences, based on the analysis of the maximum frequencies between consecutive Intrinsic Mode Functions. By combining IMFs according to their influences, the original time series can be separated into two components: one stochastic and another deterministic, as necessary to prove the stated hypothesis.

Considering the results obtained from this proof using the Nyquist-Shannon sampling theorem, we developed two new approaches to decompose time series into stochastic and deterministic components. These two new approaches are discussed in the next chapter. 


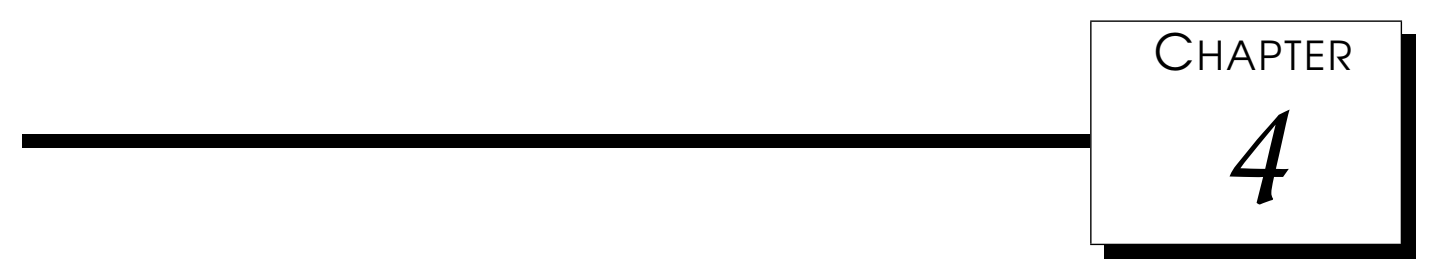

\section{Approaches Proposed to Decompose Time Series}

\subsection{Introduction}

In Chapter 3, we proved the Empirical Mode Decomposition (EMD) method extracts monocomponents (Intrinsic Mode Function - IMF) at different levels of stochasticity and, consequently, determinism. We still demonstrated the stochasticity level tends to reduce as new IMFs are extracted from time series, i.e., the first IMFs are more stochastic, whereas the last ones are more deterministic. Then, we can combine the more stochastic IMFs to form the stochastic component and the more deterministic ones to form the deterministic component. By combining IMFs according to the stochasticity/determinism levels, we go a further step to prove the hypothesis, once stochastic and deterministic components can be extracted from time series.

Based on this assumption, we designed two practical approaches to decompose time series into stochastic and deterministic components. These approaches were developed 
taking into account the stochasticity/determinism levels present in IMFs. In order to better understand the approaches, consider Figure 2.5 shown in Chapter 2. By looking at this figure, we notice different stochasticity/determinism levels in every IMF made evident a cutoff point, which can be used to separate the stochastic and the deterministic components.

Still analyzing Figure 2.5, the cutoff point is between IMFs 4 and 5, such that, all IMFs from 1 to 4 can be considered as part of the stochastic component, whereas the remaining IMFs, from 5 to 8 , are more influenced by the determinism. As a consequence, if the IMFs before this cutoff point are summed, the stochastic component is estimated. On the other hand, by summing the IMFs after this point plus the residue, the deterministic component is extracted. In order to illustrate this statement, consider Figure 4.1(a), which shows a noisy time series created by combining the deterministic and stochastic components presented in Figures 2.4(a) and 2.4(b), respectively. The EMD method was applied on this series (Figure 2.5), as consequence IMFs from 5 to 8 plus the residue were summed to form the deterministic component (IMFs from 1 to 4 are summed to compose the stochastic component). The estimated deterministic component and the expected one (Figure 2.4(a)) are shown in Figure 4.1(b).

The cutoff point, however, varies depending on the analyzed time series. Hence, aiming at automatically estimating it, two new approaches were designed. The first one combines EMD and Recurrence Plot (Eckmann et al., 1987; Marwan et al., 2007) and the second one combines EMD and Mutual Information (Darbellay and Vajda, 1999). The following sections details these approaches.

\subsection{Empirical Mode Decomposition and Recurrence Plot: First Approach}

The first approach developed to decompose time series into stochastic and deterministic components combines the Empirical Mode Decomposition method (EMD) and Recurrence Plot (RP) (Eckmann et al., 1987; Marwan et al., 2007). In summary, RP analyzes time series reconstructing it into a multidimensional space, also referred to as phase space or 


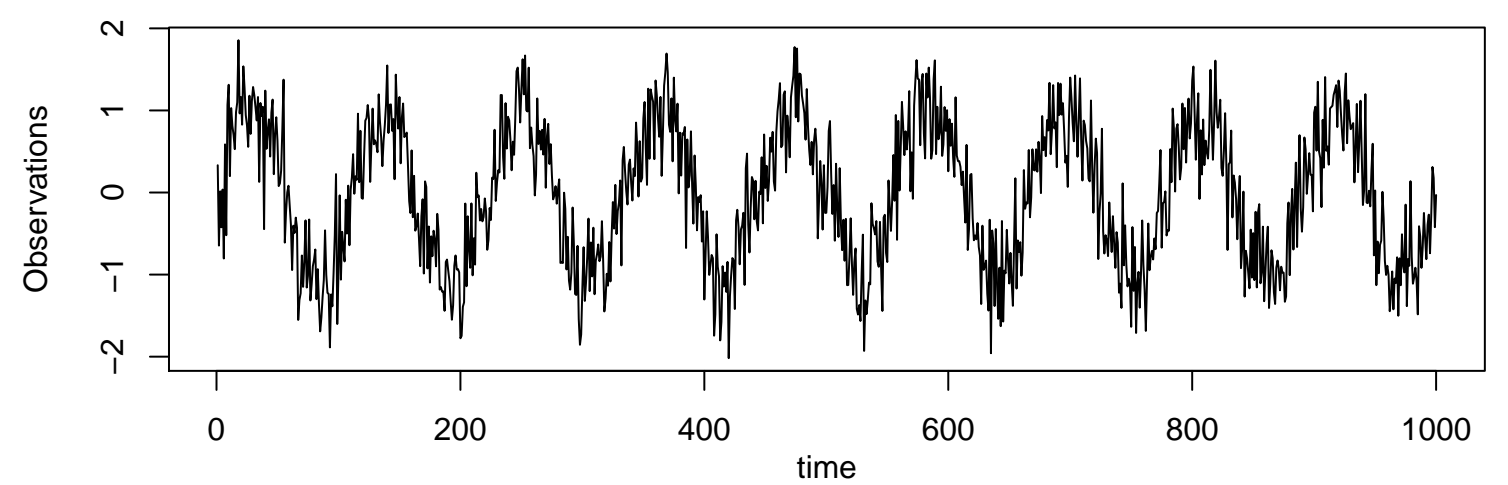

(a)

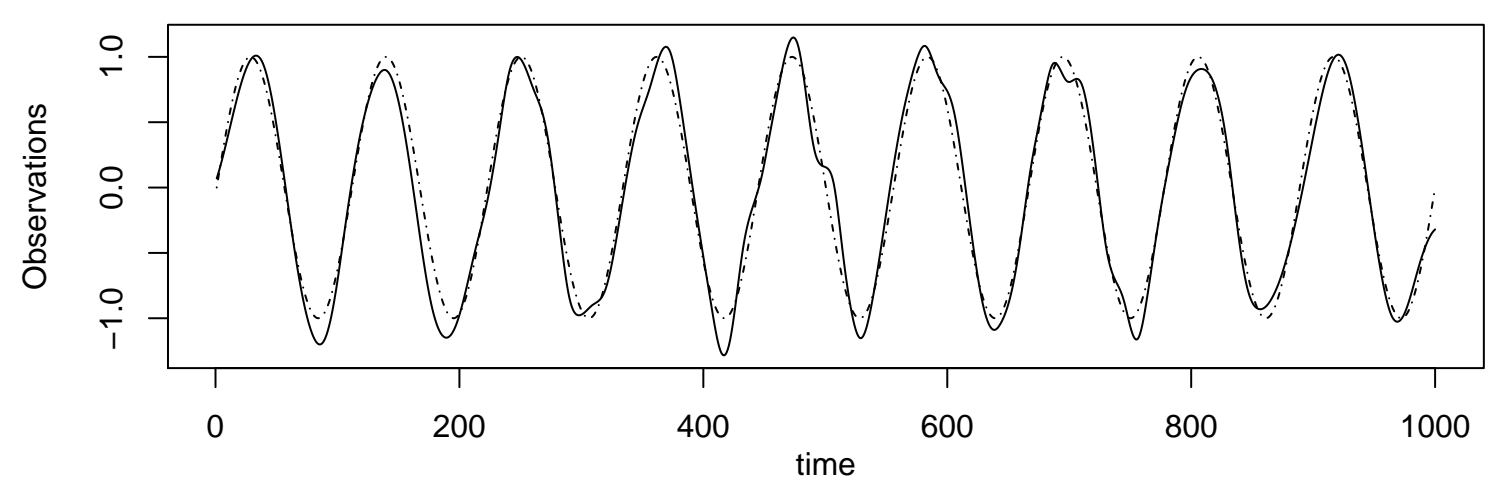

(b)

Figure 4.1: (a) Noisy time series created considering the stochastic and deterministic components as presented in Figure 2.4. (b) The dotted line represents the deterministic component $(\sin (2 \pi t))$ used to create the noisy time series. The deterministic component estimated by combining the IMFs extracted using EMD is represented by the continuous line.

time-delay coordinate space ${ }^{1}$, which maps the relationships among observations.

After this reconstruction, the relationships are organized in a two-dimensional binary matrix, called Recurrence Matrix, as defined in Equation 4.1, in which $\varepsilon$ is a distance threshold, $\|\cdot\|$ is a norm used to calculate the distance between observations, and $\Theta(\cdot)$ is a heaviside step function as defined in Equation 4.2 (Marwan et al., 2007).

$$
R_{i, j}=\Theta\left(\varepsilon-\left\|\overrightarrow{x_{i}}-\overrightarrow{x_{j}}\right\|\right)
$$

\footnotetext{
${ }^{1}$ More details about the analysis of time series into the phase space are presented in Appendix B
} 


$$
\Theta(\alpha)= \begin{cases}0, & \alpha<0 \\ 1, & \alpha \geq 0\end{cases}
$$

The structures generated by the Recurrence Matrix provide information about the time series under study (Eckmann et al., 1987; Marwan et al., 2007) such as: i) isolated points mean system states are rarely repeated, i.e., the time series is highly stochastic; and ii) diagonal lines correspond to persistent behavior, i.e., the determinism rate of a time series is directly associated to the number of diagonal lines. However, such information relies on the visual inspection of the Recurrence Plot, what is not a simple task even for humans, adding subjectivity to the estimation of stochastic and deterministic levels. In order to simplify this process and automatize it, a set of measurements called Recurrence Quantification Analysis (RQA) was developed (Zbilut and Jr., 1992; Jr. and Zbilut, 1994). Among all RQA measurements, we are particularly interested in DET, which quantifies the determinism rate of time series as defined in Equation 4.3, having $P(\varepsilon, l)$ as the frequency of diagonal lines of length $l$ present in the RP structures. This frequency is calculated using Equation 4.4.

$$
\begin{gathered}
D E T=\frac{\sum_{l=l_{\min }}^{N} l P(\varepsilon, l)}{\sum_{i=1}^{N} \sum_{j=1}^{N} R_{i, j}, \forall i \neq j} \\
P(\varepsilon, l)=\sum_{i, j=1}^{N}\left(1-R_{i-1, j-1(\varepsilon)}\right)\left(1-R_{i+l, j+l(\varepsilon)}\right) \prod_{k=0}^{l-1} R_{i+k, j+k(\varepsilon)}
\end{gathered}
$$

Considering the determinism rate provided by RQA, we developed our first approach, published in (Rios and Mello, 2013), which starts applying EMD on the time series producing a set of IMFs plus a residue. The determinism level for each IMF is, then, evaluated using RQA. In the next step, this level is compared against a minimum threshold for an IMF to be considered deterministic. If the level is higher than this threshold, the IMF is considered deterministic, otherwise it is taken as stochastic. Finally, all stochastic IMFs are summed to estimate the stochastic component. Similarly, the deterministic component is estimated by the addition of all deterministic IMFs plus the residue. Although, the residue is not considered an IMF, its influence on the time series is important because it represents the trend behavior. Consequently, by adding all IMFs, disregarding the residue, 
the original time series can be detrended, i.e., one can reconstruct the original time series changing it from nonstationary to stationary.

In order to better understand the results produced by the application of RP on IMFs, consider the Figure 2.5, which shows the IMFs extracted from the noisy time series created by combining a sine function with angular frequency equals to $2 \pi$ and a random process $\varepsilon(\mu, \sigma)$ following a Normal distribution with mean $\mu=0$ and standard deviation $\sigma=1$ (Figure 2.4). Every IMF was analyzed by RP producing a set of structures as shown in Figures 4.2 and 4.3. According to Figure 4.2, we notice the structures generated by RP for the first four IMFs (Figures 4.2(a), 4.2(c), 4.2(e), and 4.2(g)) are characterized by isolated points (Figures 4.2(b), 4.2(d), 4.2(f), and 4.2(h)), providing low determinism levels. On the other hand, the IMFs from 5 to 8 (Figures 4.3(a), 4.3(c), 4.3(e), and 4.3(f)) present higher frequencies of diagonal lines, creating recurrent structures (Figures 4.2(b), 4.2(d), 4.2(f), and 4.2(h)) and providing high determinism levels.

After analyzing the structures produced by RP, we summed the first four IMFs to form the stochastic component (Figure 4.4(a)) and the remaining ones were used to compose the deterministic component (Figure 4.4(c)). These components were also analyzed by RP as presented in Figures 4.4(c) and 4.4(d). According to these plots, we notice the resultant stochastic component is characterized by isolated points and the deterministic one presents diagonal lines and recurrence patterns as expected.

The steps involved in our first approach, referred to as EMD-RP, are detailed in (Rios and Mello, 2013) and summarized in Algorithm 1.

In Algorithm 1, threshold $\delta$ represents the minimum determinism level necessary to consider whether an IMF is deterministic or not. Hence, $\delta$ represents the confidence interval used to determine whether an IMF should be combined to form either the stochastic or the deterministic component. An empirical analysis was conducted on experimental results and it is presented in (Rios and Mello, 2013), which suggests this threshold to be equal to or greater than 0.95 .

Although this approach has presented good results (Rios and Mello, 2013), we realized the necessary parameters can influence the decomposed components. This approach relies on three parameters: i) the distance threshold $\varepsilon$ that is used to decide whether an IMF is deterministic or not; ii) the delay and embedded dimensions ( $\tau$ and $m$, respectively) 


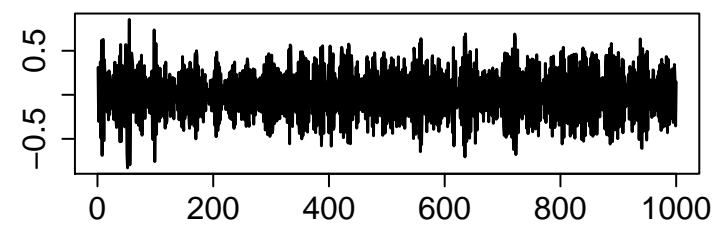

(a) IMF-1

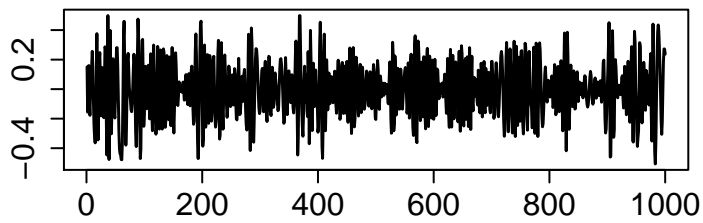

(c) IMF-2

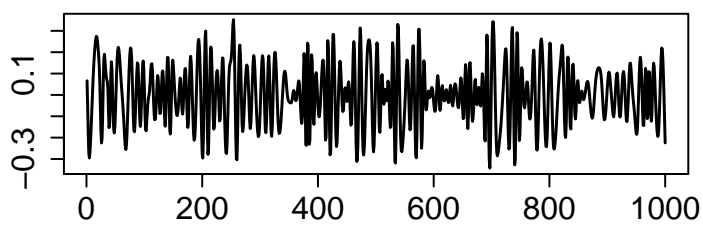

(e) IMF-3

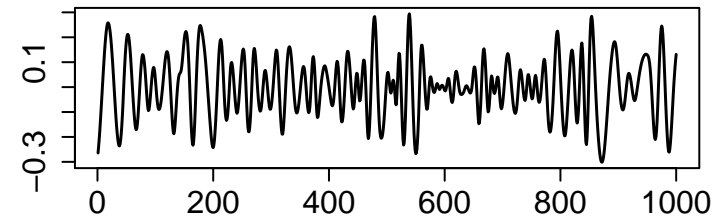

(g) IMF-4

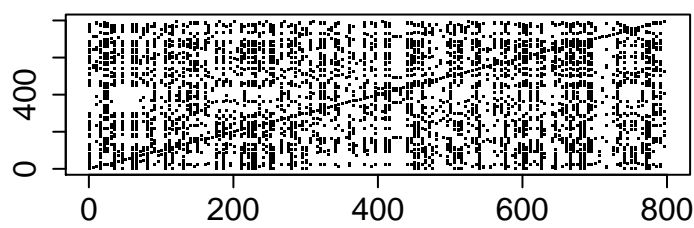

(b) $\mathrm{RP}(\mathrm{IMF}-1)$

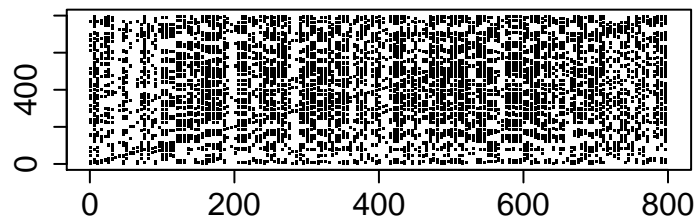

(d) RP(IMF-2)

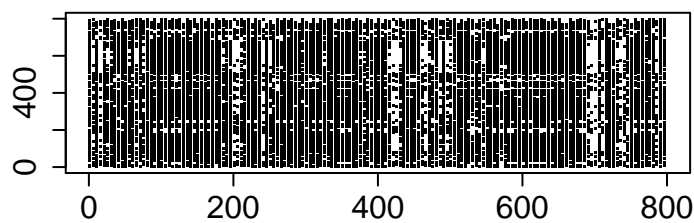

(f) RP(IMF-3)

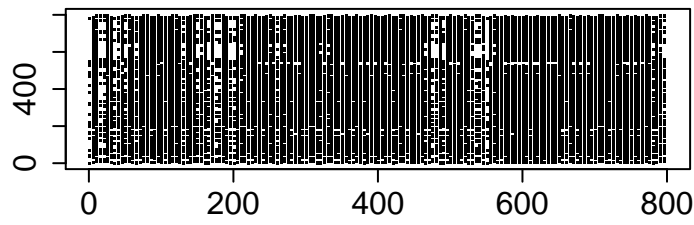

(h) RP(IMF-4)

Figure 4.2: Recurrence plot for the first four IMFs. By considering these RP structures, we notice the influence of isolated points, indicating the presence of stochastic component.

that are used to unfold the time series into the phase space. These parameters can be estimated using techniques such as Average Mutual Information (Fraser and Swinney, 1986; Abarbanel, 1996) and False Nearest Neighbors (Kennel et al., 1992). Besides these techniques, they can also be estimated using Monte Carlo simulations. However, even estimating these parameters, it is necessary a supervision, once there is no guarantee of choosing the best values for every parameter.

Aiming at overcoming this drawback, we developed a nonparametric approach combining EMD and a mutual information method, as presented in the next section. 


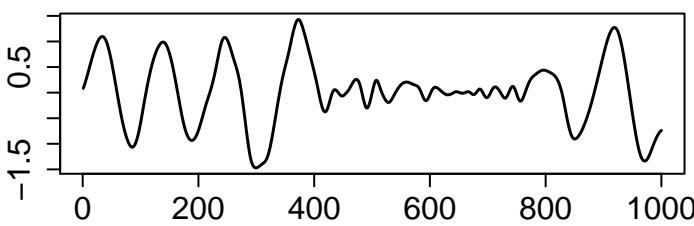

(a) IMF-5

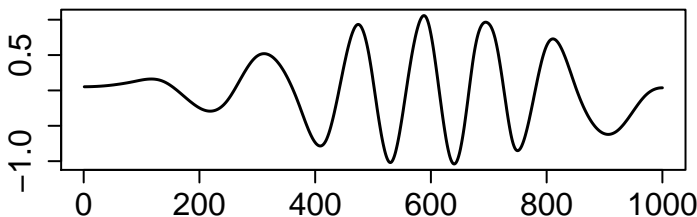

(c) IMF-6

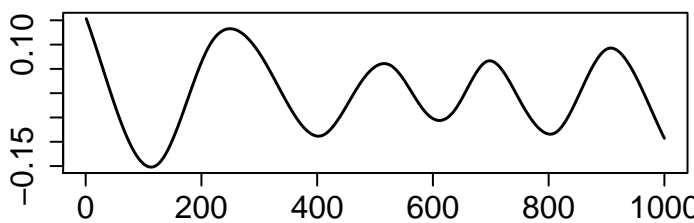

(e) IMF-7

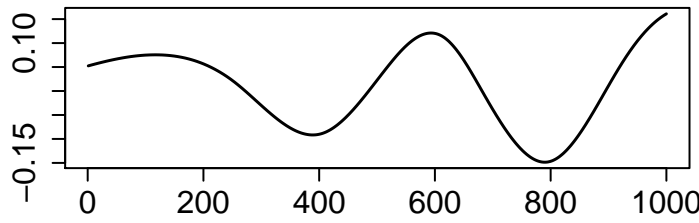

(g) IMF-8

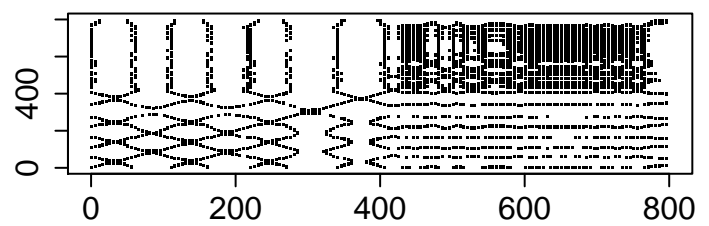

(b) $\mathrm{RP}(\mathrm{IMF}-5)$

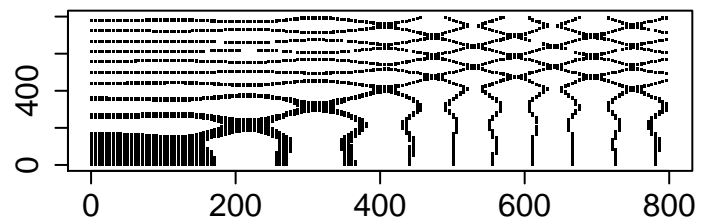

(d) RP(IMF-6)

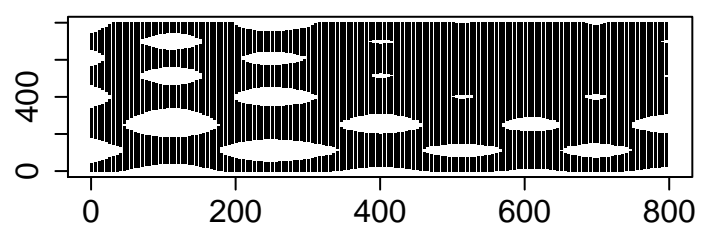

(f) RP(IMF-7)

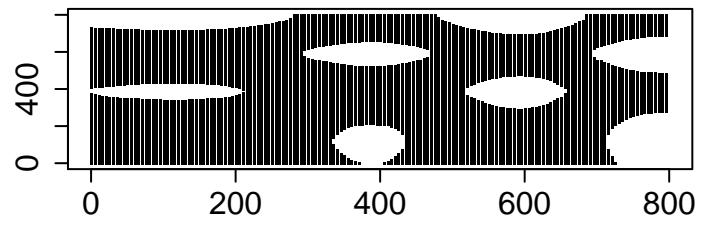

(h) RP(IMF-8)

Figure 4.3: Recurrence plot for the last four IMFs. By considering these RP structures, we notice the influence of isolated points reduces as the last IMFs are extracted from the time series, whereas the diagonal lines and recurrent patterns increases, indicating the presence of deterministic influence.

\subsection{Empirical Mode Decomposition and Mutual Information: The Second Approach}

The second approach to decompose time series into stochastic and deterministic components was designed combining EMD and Mutual Information (MI). However, before presenting this approach, it is important to briefly present an overview about Mutual Information. The concept of MI was initially introduced by Shannon (2001). According to the Information Theory area, MI estimates the information shared by two variables. For example, let $X$ and $Y$ be two random variables strictly independent, in this case their MI is zero because one variable cannot provide any information about the other (Kraskov et al., 2004). 


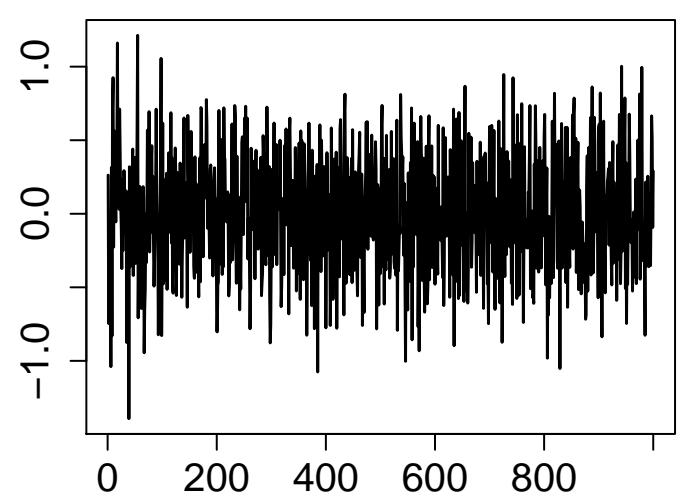

(a)

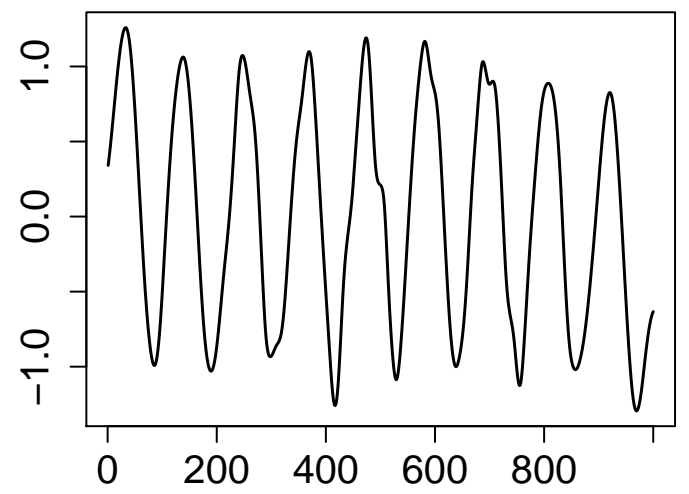

(c)

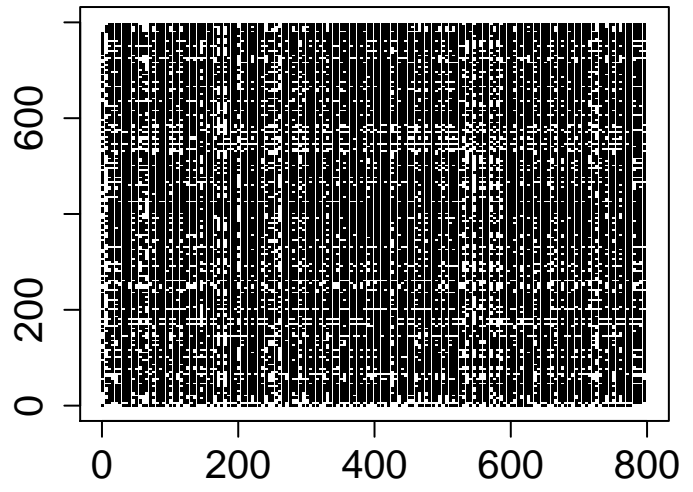

(b)

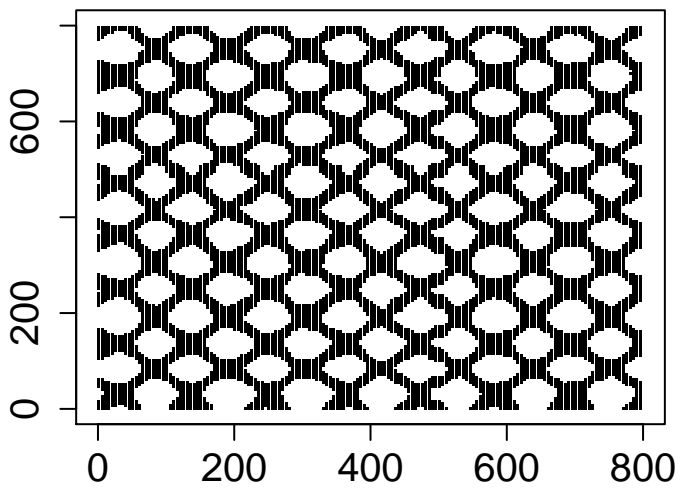

(d)

Figure 4.4: (a) Stochastic component created by the IMFs from 1 to 4 . (b) Recurrence plot for the stochastic component. (c) Deterministic component created by the IMFs from 5 to 8. (d) Recurrence plot for the deterministic component.

The mutual information between two continuous variables $X$ and $Y$ is formally presented in Equation 4.5, in which $f_{X, Y}(x, y)$ is the joint Probability Density Function (PDF) for $X$ and $Y$, and $f_{X}(x)$ and $f_{Y}(y)$ are the marginal PDF of $X$ and $Y$, respectively (Papana and Kugiumtzis, 2008).

$$
I(X, Y)=\int_{Y} \int_{X} f_{X, Y}(x, y) \log \left(\frac{f_{X, Y}(x, y)}{f_{X}(x) f_{Y}(y)}\right) d x d y,
$$

In practice, most of the mutual information estimators consider three straightforward steps. First, the dataset is partitioned into fixed-width discrete bins (cells). Then, a histogram is created considering the relative frequency of samples in each bin² (Papana and

\footnotetext{
${ }^{2}$ Several methods have been developed for estimating the number of bins and their width, but we do not discuss them in this work.
} 


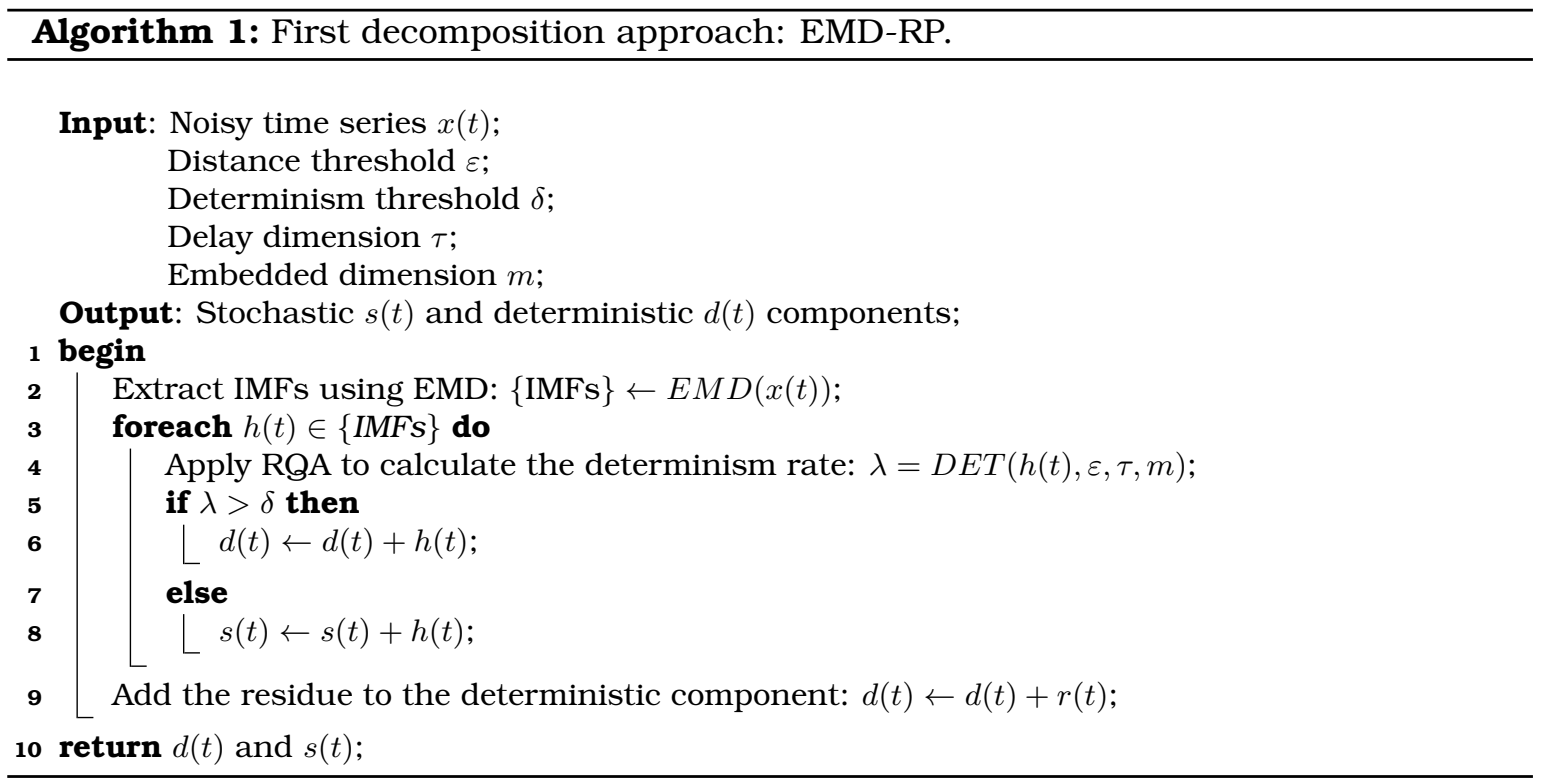

Kugiumtzis, 2008). Finally, the mutual information (Equation 4.5) is calculated on the histogram. The main issue faced by such estimators is the partitioning of data into bins, which is typically performed using a fixed width producing a high accumulated error.

Aiming at overcoming this drawback, Darbellay and Vajda (1999) presented a new method based on partitioning the data space into a finite number of nonoverlapping rectangular cells. According to the authors, instead of using fixed-width bins, the technique must continuously partition the data space until reaching a conditional independence among cells. Hence, the mutual information estimated on these partitions is closer to its theoretical value.

In summary, the execution of the Darbellay and Vajda (DV) method is based on three steps (Darbellay and Tichavský, 2000). Initially, data must be organized into one-cell partition $^{3}$. Then, the cell is partitioned into two equiprobable halves. Next, the four resultant subcells must be recursively partitioned, if the number of points in each new cell is greater than 4 and the mutual information (Equation 4.5) takes approximately the same value in each subcell. The similarity among the mutual information values in the last step is calculated using the $\chi^{2}$ "goodness-of-fit" test at $5 \%$ significance level, as depicted in (Darbellay and Tichavský, 2000). If there is no possible partitioning, in the next step, the mutual

\footnotetext{
${ }^{3}$ In order to simplify the understanding, we consider data is organized in two dimensions. An extension of this algorithm to higher dimensions is discussed in (Darbellay and Tichavský, 2000).
} 
information is calculated on the final subcells.

In the next section, we present the second decomposition approach, which combines Empirical Mode Decomposition and Mutual Information.

\subsubsection{The proposed decomposition approach}

In order to better understand the second decomposition approach, consider a time series $x(t)=\{x(1), x(2), \ldots, x(T)\}$ having $T$ observations. To obtain the stochastic and deterministic components, $x(t)$ is initially decomposed using EMD, $\mathcal{E}(\cdot)$, which returns a set of IMF monocomponents, i.e., $h_{n}(t), \forall n \in\{1,2, \ldots, N\}$ (see Section 2.2), in which $N$ represents the number of IMFs extracted from $x(t)$, plus a residue $r(t)$. It is important to highlight that every IMF is a time series in itself, whose length ${ }^{4}$ is equal to the original one, i.e., $\left|h_{n}(t)\right|=|x(t)|=T$. Moreover, the total number of IMFs returned by EMD depends on the time series.

$$
\mathcal{E}(x(t))=\left\{h_{1}(t), h_{2}(t), \ldots, h_{N}(t), r(t)\right\}
$$

In the next step, Fourier Transform $\mathcal{F}(\cdot)$ is applied on every extracted IMF (Equation 4.7), but the residue, once we expect it to be deterministic because it only contains the time series trend. There are several algorithms available to calculate the Fourier Transform. In this work, we adopted the Fast Fourier Transform (FFT) due to its low time complexity. Then, for every IMF $h_{n}(t)$, we obtain a set of complex coefficients $C_{n}(t)$ as presented in Equation 4.7 .

$$
C_{n}(t)=\mathcal{F}\left(h_{n}(t)\right)
$$

Hence, the output produced by $\mathcal{F}\left(h_{n}(t)\right)$ is a set of complex coefficients $C_{n}(t)=$ $\left\{c_{n, 1}, c_{n, 2}, \ldots, c_{n, k}, \ldots, c_{n, T}\right\}$, such that $c_{n, k}$ is calculated by Equation 4.8 . The length of $C_{n}(t)$ is also equal to the original time series, i.e., $\left|C_{n}(t)\right|=\left|h_{n}(t)\right|=|x(t)|=T$.

$$
c_{n, k}=\sum_{t=1}^{T} h_{n}(t) \cdot e^{-i 2 \pi \frac{k}{T} t}, \quad \forall k \in\{1,2, \ldots, T\}
$$

\footnotetext{
${ }^{4}$ Operator $|\cdot|$ corresponds to cardinality.
} 
After obtaining the coefficients for every IMF, we calculate the phase components. For this purpose, the arctangent function is applied on the ratio between the imaginary $\Im(\cdot)$ and real $\Re(\cdot)$ parts of complex coefficients as presented in Equation 4.9. As previously presented, $n$ represents the IMF index and the length of $\theta_{n}(t)$ is also equal to the original time series, i.e., $\left|\theta_{n}(t)\right|=\left|C_{n}(t)\right|=\left|h_{n}(t)\right|=|x(t)|=T$.

$$
\theta_{n}(t)=\arctan \left(\frac{\left.\Im\left(C_{n}(t)\right)\right)}{\left.\Re\left(C_{n}(t)\right)\right)}\right), \quad \forall n \in\{1,2, \ldots, N\}
$$

Next, the Darbellay-Vajda Mutual Information (DV) method $I(X, Y)$ is applied on the phases of consecutive IMFs $X$ and $Y$ (Equation 4.10) in order to compare their phases $h_{p}(t)$ and $h_{p+1}(t)$, such as $\forall p \in\{1,2, \ldots, N-1\}: \theta_{1}(t)$ versus $\theta_{2}(t), \theta_{2}(t)$ versus $\theta_{3}(t), \ldots, \theta_{p}(t)$ versus $\theta_{p+1}(t)$. The main advantage of applying the Darbellay-Vajda Mutual Information method on the phases obtained from the Fourier Transform is the possibility of detecting different temporal patterns, suggesting the cutoff point, what is not evident when applying this method directly on the IMFs. In this sense, deterministic IMFs present phases with similar behaviors, providing high mutual information values, once there is a strong entropy among them.

$$
v_{p}=I\left(\theta_{p}(t), \theta_{p+1}(t)\right)
$$

After executing the DV method on every pair of consecutive IMFs, the resultant values are stored into a vector $\mathrm{v}$ as presented in Equation 4.11.

$$
\mathbf{v}=\left(\begin{array}{c}
v_{1} \\
v_{2} \\
\vdots \\
v_{p} \\
\vdots \\
v_{N-1}
\end{array}\right)
$$

By comparing two stochastic IMFs, the obtained Mutual Information is close to zero, once the correlation between them is low. On the other hand, the comparison between deterministic IMFs tends to provide higher MI values. Hence, by considering the mean 


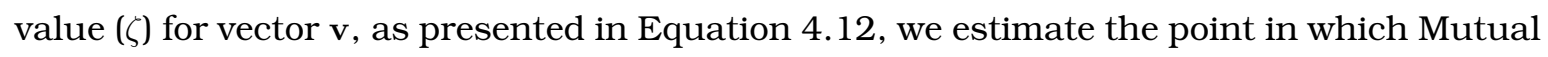
Information values change abruptly, this cutoff point is used to separate the stochastic and deterministic components.

$$
\zeta=\frac{1}{N-1} \sum_{p=1}^{N-1} v_{p}
$$

Then, we look for the value in vector $\mathbf{v}$ that is immediately lower than mean $\zeta$, here referred to as $v_{z}$, which is used as cutoff point. Every $h_{i}(t), \forall i \in\{1,2, \ldots, z\}$, is considered stochastic, while all $h_{j}(t), \forall j \in\{z+1, z+2, \ldots, N\}$, plus the residue are taken as deterministic, as presented in the example in Section 2.2. These two components are, formally, defined by Equations 4.13 and 4.14.

$$
\begin{gathered}
\mathbf{s}(t)=\sum_{n=1}^{z} h_{n}(t) \\
\mathrm{d}(t)=\sum_{n=z+1}^{N} h_{n}(t)+r(t)
\end{gathered}
$$

It is important to highlight that the IMFs extracted by EMD are not modified, assuring the initial condition defined in Equation 2.3, such that the sum of IMFs plus residue results in the original time series. Hence, by summing the stochastic and deterministic components, the original time series is also obtained, i.e., $x(t)=s(t)+d(t)$. All decomposition steps involved in our second approach, referred to as EMD-MI, are summarized in Algorithm 2.

\subsubsection{Determinism rate}

Assessing the degree of determinism in a time series is a very important step in the analysis process, helping to choose more appropriate techniques to model and understand data behavior (Rios and Mello, 2013). The most commonly considered approach to quantify determinism is the Recurrence Plot (RP) method (Marwan et al., 2007). However, RPs rely on some critical parameters that strongly influence the determinism quantification.

Aiming at overcoming this issue, we designed a new measure called Determinism Rate, $R_{\text {det }}$, which is computed on two components $s(t)$ and $d(t)$ obtained by our second decom- 


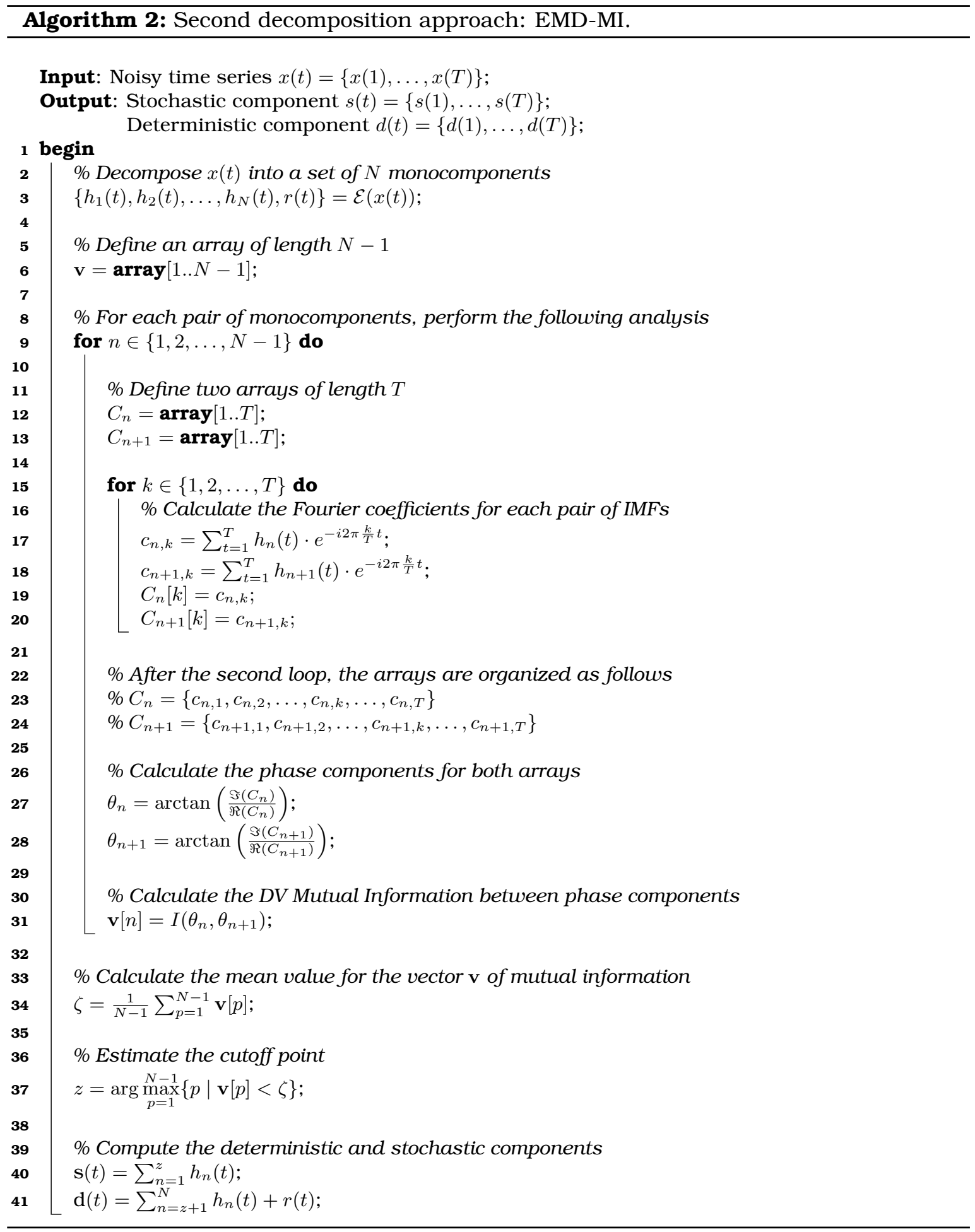


position approach. This measure $R_{\text {det }}$ computes the mean influence of the deterministic component $d(t)$ in terms of the original time series, $x(t)=s(t)+d(t)$, as stated in Equation 4.15. In this equation, $\epsilon$ is a very small constant, $10^{-15}$, which is useful to avoid undefined division, i.e., when the deterministic component in a given time instant $t$ is zero and there is no stochastic influence.

$$
R_{\mathrm{det}}=\frac{1}{T} \cdot \sum_{t=1}^{T} \frac{d(t)^{2}+\epsilon}{d(t)^{2}+s(t)^{2}+\epsilon}
$$

This equation provides values in the range $(0,1]$. As this value approaches to one, the time series is more deterministic. On the other hand, if the value is close to zero, the time series has a strong stochastic influence. Alternatively, the Stochasticity Rate is obtained by applying the following equation: $R_{\text {sto }}=1-R_{\text {det }}$.

In general, $R_{\text {det }}$ can be interpreted as a measurement to evaluate the energy or influence of the deterministic component on time series. To avoid miscalculating this evaluation, the deterministic and stochastic components were squared, transforming the component influences from negative to positive, i.e., the influences are always positively quantified. In order to better understand the positive and negative influences, consider Figure 4.5. Analyzing Figure 4.5(a), we observe that the $x(t)$ value is influenced by a positive noise, which is added to the deterministic component. On the other side, in Figure 4.5(b), the $x(t)$ value is influenced by a negative noise, reducing the determinism value. Finally, it is worth mentioning that $s(t)^{2}+d(t)^{2} \neq x(t)^{2}$, i.e., the deterministic rate is not calculated according to the original time series. It is calculated considering the deterministic and stochastic influences present in the original time series, instead.

\subsection{Concluding remarks}

In this chapter, we presented two new approaches developed to decompose noisy time series into stochastic and deterministic components. These new approaches and the formal definition presented in the previous chapter supported the first part of the hypothesis presented in the introduction, in which we state stochastic and deterministic components can be separated from time series.

The second part of the hypothesis states better models can be obtained when these 


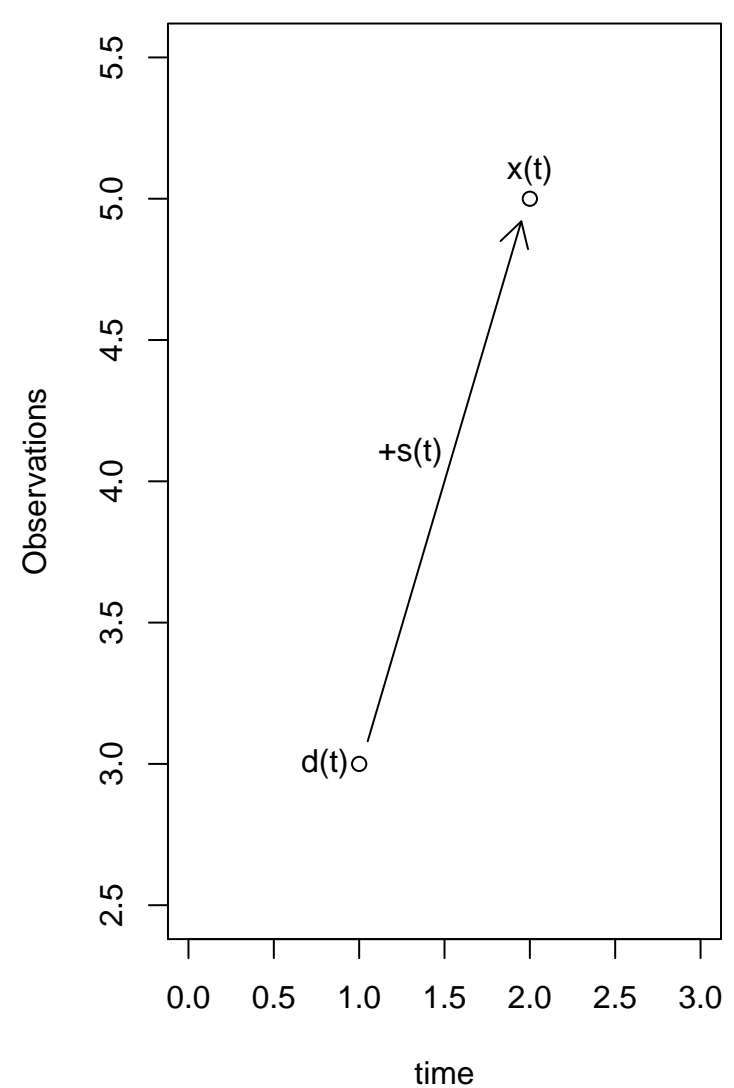

(a)

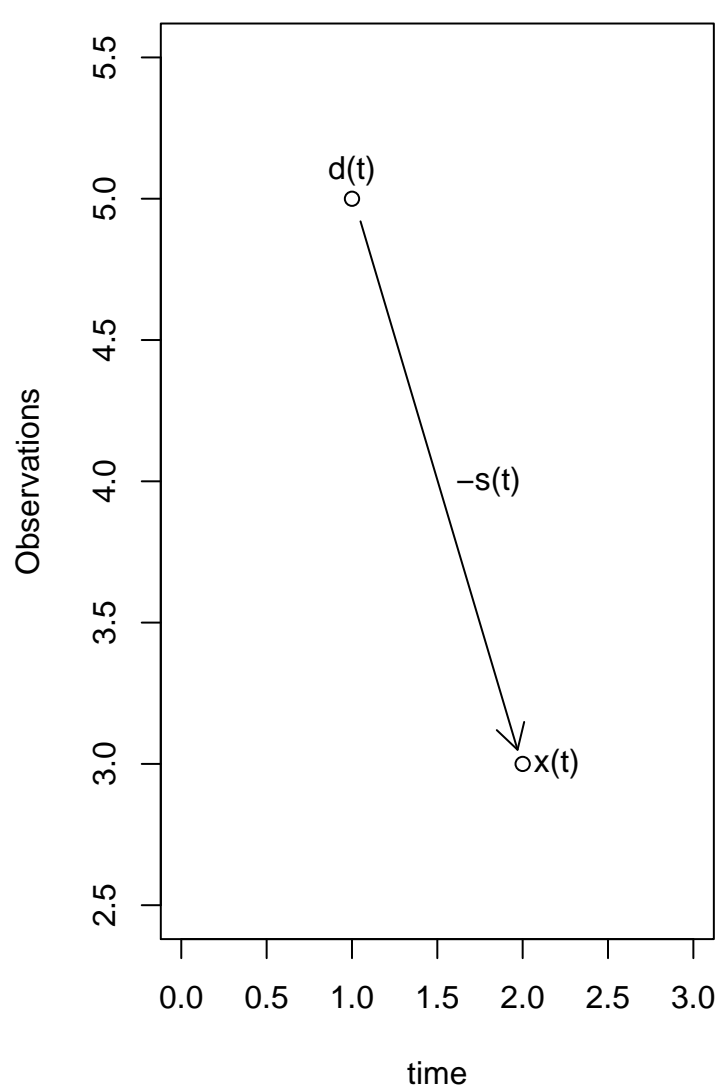

(b)

Figure 4.5: Example of the stochastic influence on a time series: (a) $x(t)$ is influenced by a positive noise; (b) $\mathrm{x}(\mathrm{t})$ is influenced by a negative noise.

components are separately analyzed. Aiming at proving this second part, we performed two sets of experiments. In the first set, we applied the first proposed approach EMD-RP on synthetic time series. The use of synthetic time series was important because they provide a controlled scenario in which the expected behavior is known a priori. In this set of experiments, we evaluated not only the EMD-RP approach but also the importance of modeling each individual component.

The second set of experiments was composed of time series obtained from real-world applications. Due to the huge amount of time series in this experiments, only the EMDMI approach was applied on them because EMD-RP requires supervision, making its use unfeasible. No experiment was performed comparing the two approaches because, as empirically observed, both produce similar decomposition results. In addition, one can select the best approach based on his/her ability to supervise the whole decomposition 
process.

Finally, it is important to highlight that although EMD-MI is a very attractive approach to decompose time series due to its nonparametric manner, in situations such that the execution and analysis time are not a restriction, it is worth adopting the EMD-RP approach once the cutoff point can be better chosen by selecting the best decomposition parameters. 


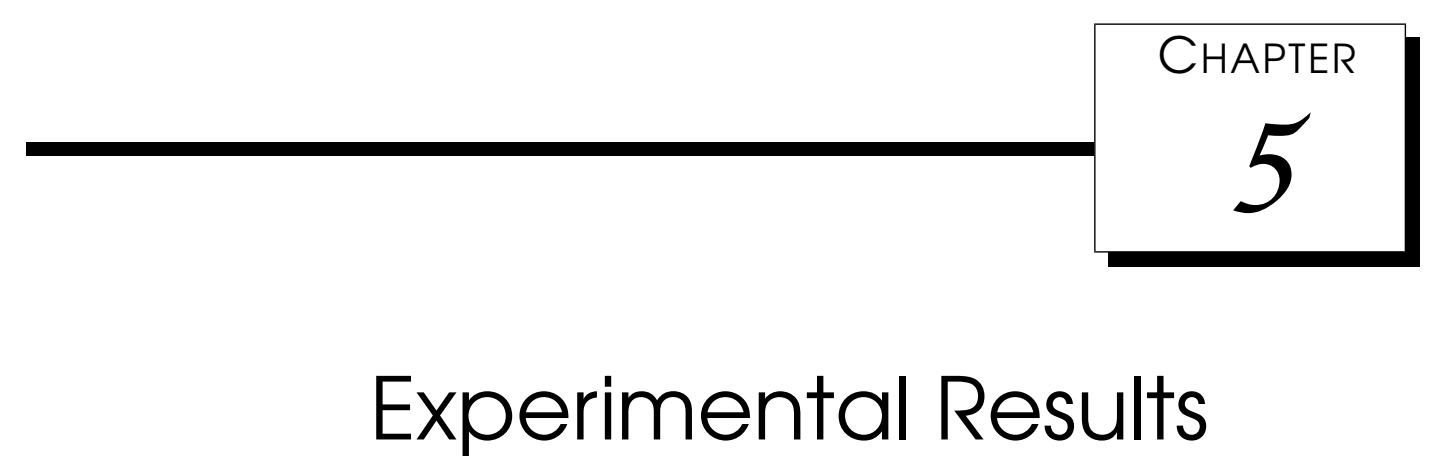

\subsection{Introduction}

As previously discussed, the main goal of this thesis is to prove the hypothesis presented in Chapter 1. In this sense, this proof was conducted in two steps. In the first one, discussed in Chapter 3, we formally proved time series can be decomposed into stochastic and deterministic components. In the second step, presented in this chapter, we analyzed the advantages of separately modeling the decomposed components. For this, we applied the approach that combines Empirical Mode Decomposition and Recurrence Plot (EMD$\mathrm{RP}$ ), presented in Section 4.2, on synthetic time series. We decided to use synthetic data in this chapter because they provide a controlled scenario, which better supports conclusions.

In this step, we only analyzed EMD-RP because it permits understanding the whole decomposition process and the importance of every parameter. Afterwards, we present results considering the EMD and Mutual Information (EMD-MI) approach (see Section 4.3) applied on real-world time series. We remember the basic difference between EMD-RP and EMD-MI is that the later is nonparametric and requires no supervision during series 
decomposition. All discussion presented in this chapter was based on the results published in (Rios and Mello, 2013).

\subsection{Experimental setup}

This chapter presents an analysis on the advantages of separately modeling the stochastic and deterministic components extracted from time series. This analysis was performed on synthetic time series characterized by additive noise, which were organized into three different scenarios: i) a time series composed of a sine function, representing the deterministic influence, plus a stochastic component following a Normal distribution; ii) two time series composed of a sine function, representing deterministic influences, plus two different stochastic components following an autoregressive moving average model and a colorednoise process; and iii) a set of ten time series combining the Lorenz system, representing the deterministic component, plus a white noise process, representing the stochastic component, following a Normal distribution with mean equals to zero at different standard deviations.

The analysis presented in this chapter was performed based on the forecasting domain, which aims at analyzing past observations to estimate the lead time, i.e., future observations (see Figure 2.2). Hence, every time series was divided into two folds over time ${ }^{1}$. The first fold was formed by the first $95 \%$ of observations to adjust models, whereas the second fold, composed of the remaining $5 \%$, was used to evaluate the prediction accuracy.

Finally, the analysis proposed in this chapter is executed comparing the accuracy of models adjusted on undecomposed (i.e., original) time series and individual models adjusted on decomposed stochastic and deterministic components. At last, we combined the stochastic and the deterministic models to form the hybrid model which was also considered in evaluations and allowed to prove the last part of our hypothesis.

The models considered in the scenarios were: i) Radial Basis Function (RBF); ii) Polynomial; and iii) Autoregressive (AR). The Radial Basis Function (Marsland et al., 2002; Hegger et al., 2009) was designed to model deterministic time series. This function performs a regression analysis according to a weighted distance among observations in order

\footnotetext{
${ }^{1}$ In the time series analysis area, the $k$-fold cross-validation approach cannot be applied to evaluate models due to temporal dependencies among observations. Torgo (2010) presents an adapted approach that permits applying 10 -fold cross-validation in temporal data. However, in this thesis, this adapted approach was not considered.
} 
to approximate Gaussian functions. In general, RBF estimates a function $\hat{f}($.) to generate similar observations to the original time series. This function is defined by Equation 5.1, in which $k$ represents the number of centers, $w_{i}$ is the weight associated to every center, and $K($.$) represents a function, typically a Gaussian function, whose values reduce as distance$ $d($.$) between the observed and expected observations increases.$

$$
\hat{f}\left(x_{n}\right)=w_{0}+\sum_{i=1}^{k} w_{i} K\left(d\left(x_{i}, x_{n}\right)\right)
$$

The prediction performed by this method depends on the number of considered Gaussian functions, whose radial function centers and variance (typically represented as $\sigma^{2}$ ) are adjusted based on observations.

Similarly to RBF, the Polynomial model attempts to find out an approximation function to time series observations. This approximation function is defined by $\pi_{i} f_{N}: \mathbb{R}^{m} \rightarrow \mathbb{R}, i=$ $1, \ldots, m$, in which $m$ represents variables with degree at most $d$. The parameterization for this function is performed to minimize equation $\sum_{n=1}^{N-1}\left(\pi_{i} x_{n+1}-\pi f_{N}\left(x_{n}\right)\right)^{2}$ (Casdagli, 1989).

Finally, the Autoregressive model (AR) (Box et al., 1994) is indicated to represent stochastic time series. AR is defined in terms of a linear combination of past values and noises. This model is defined by $x_{t}=\phi_{1} \cdot x_{t-1}+\phi_{2} \cdot x_{t-2}+\cdots+\phi_{p} \cdot x_{t-p}+\varepsilon_{t}$, in which $\left\{\phi_{p}\right\}$ are constants and $\varepsilon_{t}$ represents a purely random process with mean $E\left(X_{t}\right)=0$ and variance $\operatorname{var}\left(X_{t}\right)=\sigma^{2}$ (Box et al., 1994).

After modeling the synthetic time series, new observations are predicted and compared against the expected ones using a new measurement as presented in next section.

\subsection{MDDL measurement}

During experiments, we observed most of measurements commonly adopted to evaluate prediction results only analyzes the differences between expected and predicted observations, but they do not verify the global behavior, which can present similar trend and seasonality. To address this issue, we developed a new measurement called Mean Distance from the Diagonal Line (MDDL) (Rios and Mello, 2013), which is based on Dynamic Time Warping (DTW) (Tormene et al., 2009). By using DTW, we find the necessary time displacements to obtain the best synchronization between these two series and, afterwards 
we evaluate their similarities using MDDL.

Hence, the MDDL measurement starts calculating DTW for the expected and predicted time series, producing a matrix containing a warping path. The first and last elements of the warping path are connected through a straight diagonal line, as shown in Figure 5.2. At last, the MDDL measurement is obtained by computing the absolute area between the warping path and the diagonal line. This area is illustrated as the shaded area in Figure 5.2. By analyzing this figure, we conclude that as the absolute area between the warping path and the diagonal lines increases, the similarity between the expected and predicted time series decreases (Rios and Mello, 2013).

In this sense, the MDDL score varies in interval $\left[0, \int_{a}^{b}|f(x)| d x\right]$, such that $f(x)$ is the MDDL function used to calculate the warping path, $a$ and $b$ are the first and last elements in the path, and $d x$ corresponds to differentials computed on the diagonal line. By considering this interval, we state MDDL scores vary from zero, which represents a perfect prediction, to the maximum area formed by the warping path and the diagonal line. In the worst case, when the predicted and expected observations are completely different, MDDL returns the area of the right isosceles triangle, whose sides are formed by $N$ expected and $N$ predicted observations, whereas the hypotenuse is formed by the diagonal line. Consequently, we can rewrite this interval as $\left[0,+\frac{\left(N^{2}-N\right)}{2}\right]$ (Rios and Mello, 2013). In practical situations, the number of observations is finite, thus we can simply calculate the Euclidean distance among the warping path and the diagonal line (Rios and Mello, 2013).

In order to exemplify the advantages of the MDDL measurement, we initially designed an example using a time series $x(t)$ generated by a sine function with amplitude equals to 0.5 and angular frequency equals to $2 \pi$, which represents the expected observations. Then, we created two time series to simulate predicted observations: i) a time series $p_{1}(t)$ created by the combination of $x(t)$ and a noise following a Normal distribution with mean equals to zero and standard deviation equals to 0.4 ; and ii) a time series $p_{2}(t)$ having a single value based on the mean observation values of $x(t)$ (Rios and Mello, 2013). These three time series are shown in Figure 5.1.

After looking at the resultant time series, we can conclude the best model is the one used to produce $p_{1}(t)$. However, by calculating the DTW distance between expected and predicted observations, we obtained the following results: $\operatorname{DTW}\left(x(t), p_{1}(t)\right)=91.17$ and $\operatorname{DTW}\left(x(t), p_{2}(t)\right)$ 


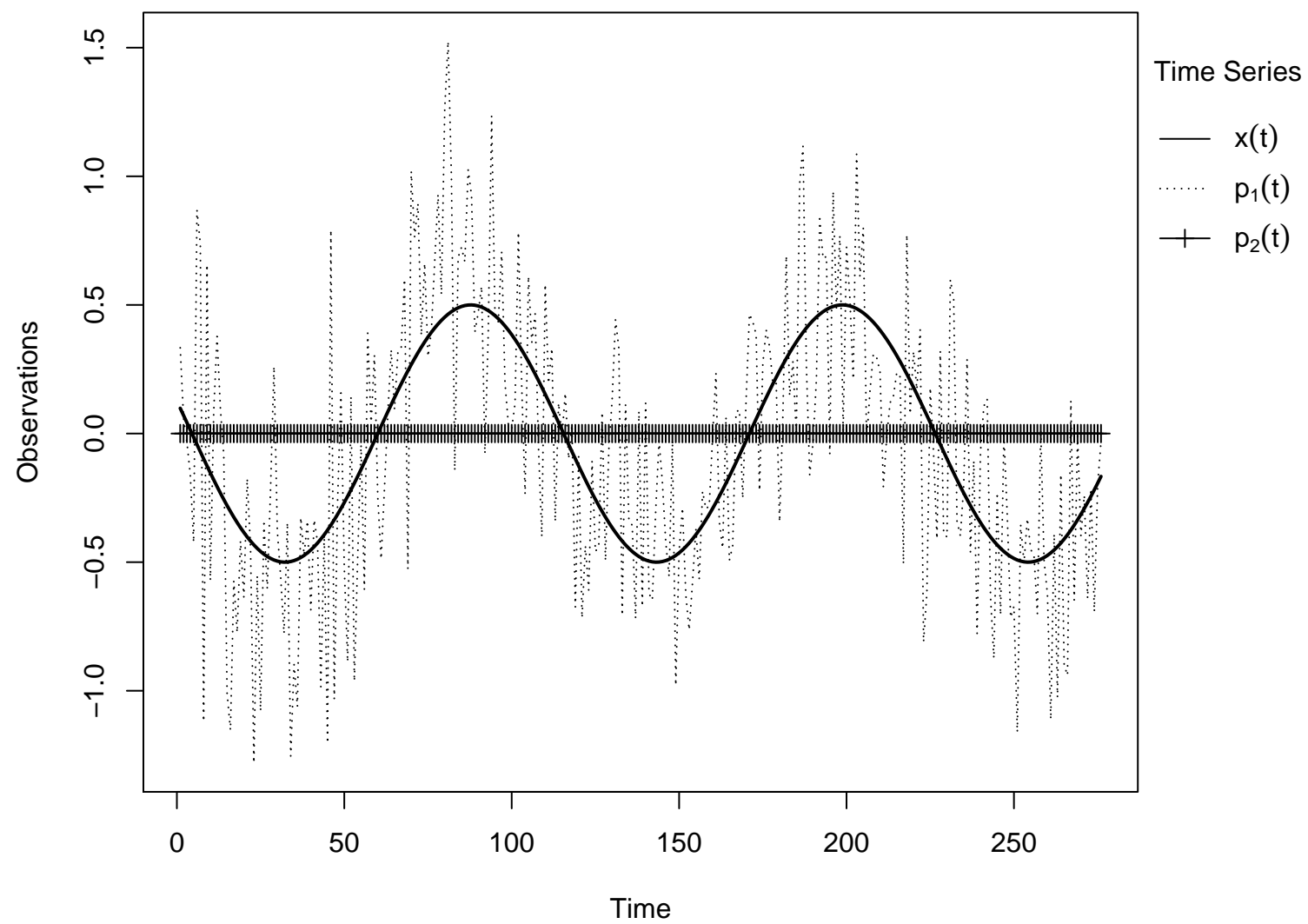

Figure 5.1: The continuous curve represents the expected sine values $x(t)$, and the other two curves represent the predicted time series $p_{1}(t)$ and $p_{2}(t)$ (adapted from (Rios and Mello, 2013)).

$=88.13$. Considering only these values, we infer the best method is the one used to predict the mean values. Nonetheless, considering MDDL measurement, we can achieve the same conclusion as expected by the visual inspection (Rios and Mello, 2013): $\operatorname{MDDL}\left(x(t), p_{1}(t)\right.$ ) $=3.55$ and $\operatorname{MDDL}\left(x(t), p_{2}(t)\right)=51.24$. The MDDL charts for the time series are presented in Figure 5.2.

It is important to highlight that this problem is faced not only by DTW but also by several measurements that individually compare time series observations, such as the pointwise Euclidean distance.

Another important characteristic of the MDDL measurement is related to the stability of its results, which are not influenced by the number of series observations. In this measurement, scores are more influenced by the general behavior than the time series 


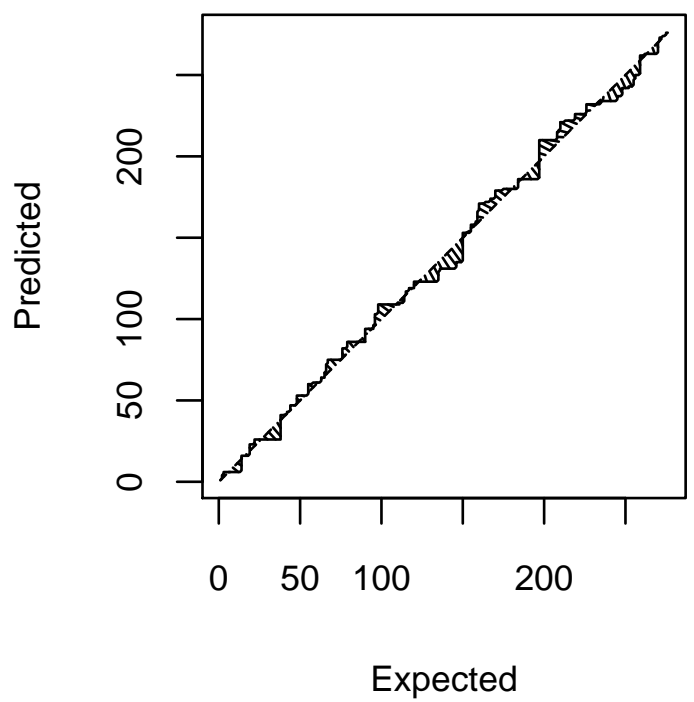

(a)

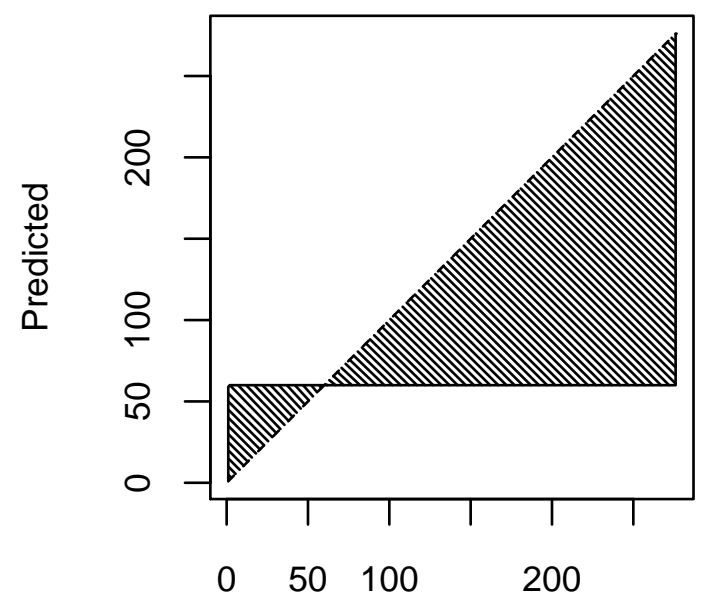

Expected

(b)

Figure 5.2: The MDDL plot shows the differences of the expected time series $x(t)$ in relation to: (a) the predicted noisy observations $p_{1}(t)$; and (b) mean observations $p_{2}(t)$. The solid line represents the warping path and the diagonal line is represented by the dashed one. By looking at these plots, it is possible to notice the shaded area in the first case is much smaller, indicating that the model used to predict $p_{1}(t)$ is better than the one used to predict $p_{2}(t)$ (adapted from (Rios and Mello, 2013)).

length. For instance, consider the same time series $x(t)$ and $p_{1}(t)$ presented in Figure 5.1. By applying the DTW measurement on these time series with 100 and 1000 observations, we obtained the following scores, respectively: DTW $\left(x(t), p_{1}(t)\right)=36.58$ and DTW $\left(x(t), p_{1}(t)\right)$ $=337.05$. On the other hand, the MDDL measurement applied on the same time series produces the scores: $\operatorname{MDDL}\left(x(t), p_{1}(t)\right)=5.64$ and $\operatorname{MDDL}\left(x(t), p_{1}(t)\right)=5.41$. However, if no similar behavior is detected between time series, MDDL resembles the DTW measurement, facing the same problem related to the time series length.

Due to this stability and capability of evaluating general similarities between time series, the MDDL measurement was adopted to analyze the predictions presented in the following sections.

\subsection{Scenario I: Sine function plus white noise}

In this first scenario, we created a noisy time series combining a sine function (Figure 5.3(a)) and a white noise process (Figure 5.3(c)), which simulates the deterministic and 
stochastic components, respectively (Rios and Mello, 2013). The sine function was defined with amplitude equals to one and angular frequency equals to $\frac{\pi}{4}$ and the stochastic process followed a Normal distribution with mean equals to zero and standard deviation equals to 0.4 .

We started reconstructing these time series in the phase space (or time-delay coordinate space) ${ }^{2}$, which was performed by estimating the embedding and separation dimensions. The embedding dimension was estimated using the False Nearest Neighbors (FNN) method (Kennel et al., 1992) and the separation dimension was estimated using the Average Mutual Information (AMI) method (Takens, 1980; Fraser and Swinney, 1986; Abarbanel, 1996). As seen in Figure 5.3(b), the original time series presents a well-defined attractor. On the other hand, after adding the stochastic component, the attractor behaves like a cloud of dispersed points (see Figure 5.3(d)).

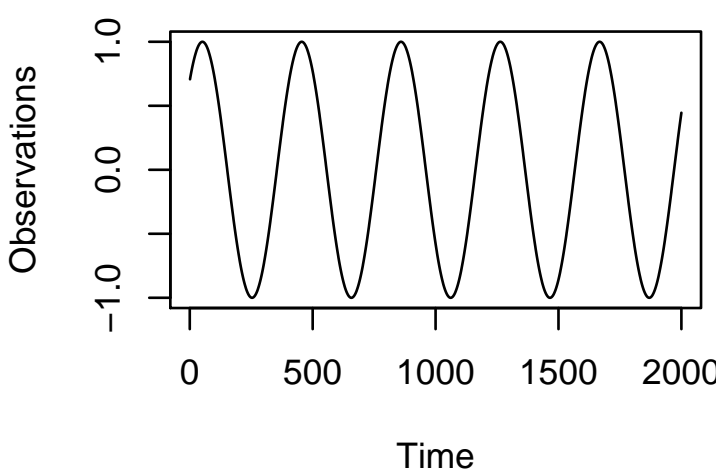

(a) Sine time series

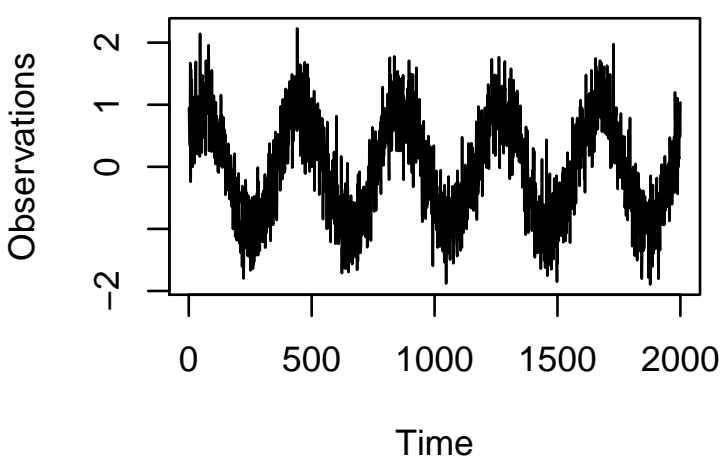

(c) Noisy time series

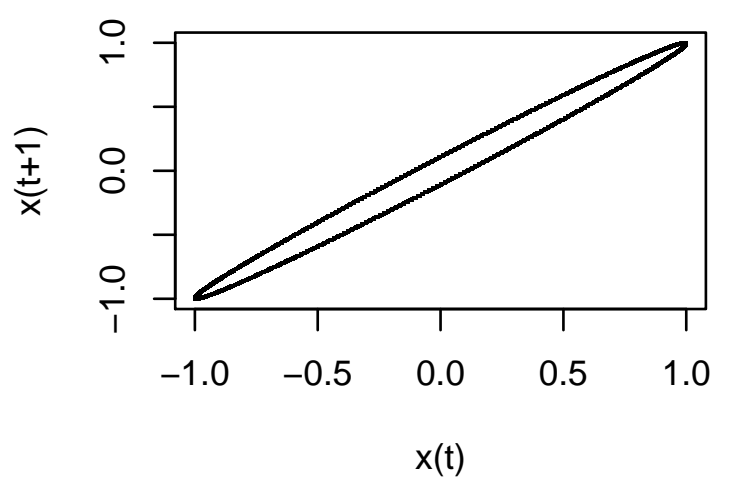

(b) Unfolded sine time series

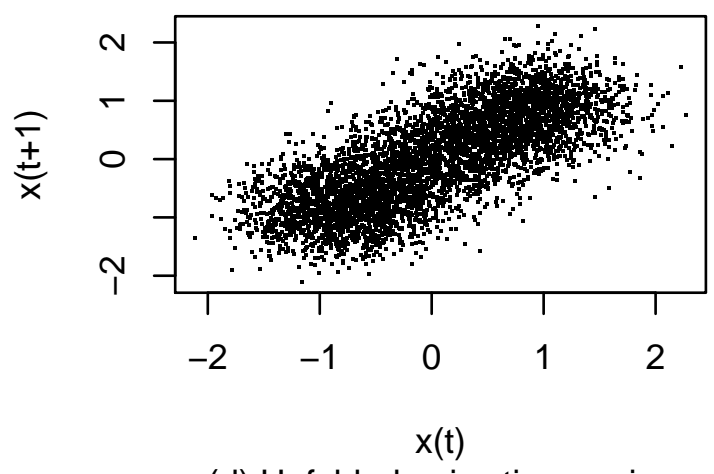

(d) Unfolded noisy time series

Figure 5.3: (a) Time series generated by sine function. (b) The sine time series unfolded in phase space. (c) The noisy time series generated by sine function plus the white noise process. (d) The noisy time series unfolded in phase space.

\footnotetext{
${ }^{2}$ Details about the study of time series in the phase space is presented in Appendix B.
} 
In order to better visualize the stochastic influence, we plotted the qqplot and boxplot for the original and the noisy time series (Figure 5.4). The qqplot chart is, commonly, used to determine whether data follows a Normal distribution, i.e., the time series observations evolve around a diagonal line under the same variance $\sigma^{2}$ and mean $\mu$. Therefore, considering Figure 5.4(c), we conclude that the noisy time series follows a Normal distribution, i.e., the original behavior of the deterministic time series was changed after adding the observations produced by the white noise process.

The boxplot chart, on the other hand, allows us to study the time series by using quantiles and the variations among observation values. Hence, by analyzing Figures 5.4(b) and 5.4(d), we notice the addition of noise has changed the sine time series, varying its scale and increasing the distance between extrema values.

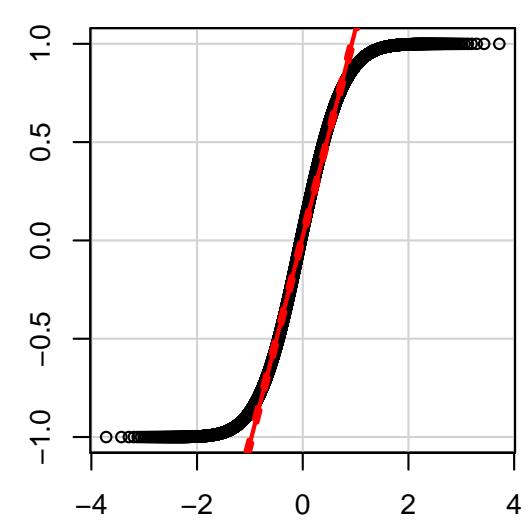

(a) QQPlot of the original series

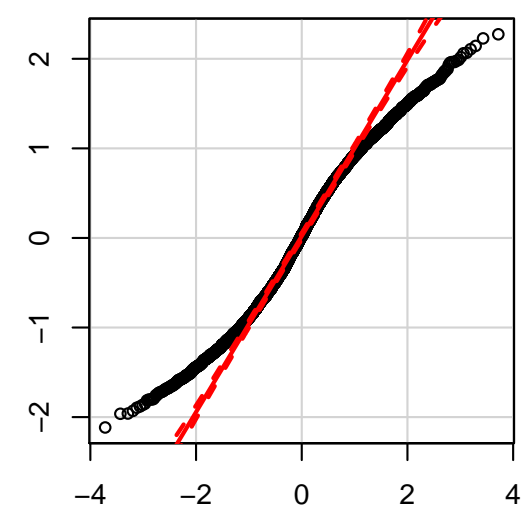

(c) QQPlot of the noisy series

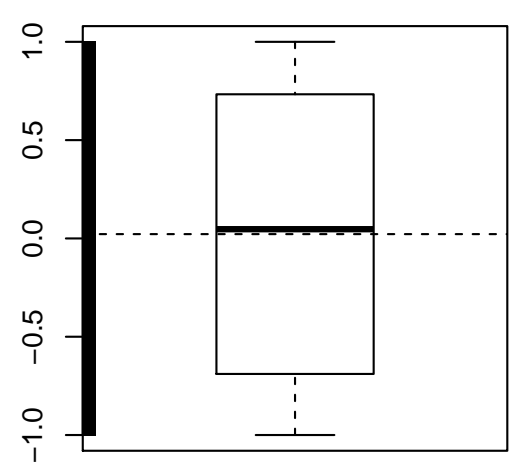

(b) Boxplot of the original series

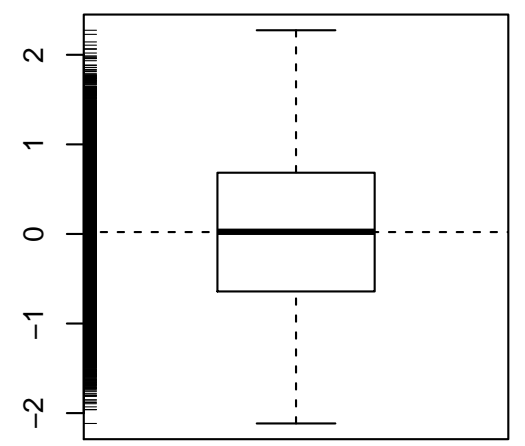

(d) Boxplot of the noisy series

Figure 5.4: Considering the qqplot and boxplot charts, one can notice the stochastic component strongly influenced the general behavior of the sine time series. 
Afterwards, we applied the EMD-RP approach (see Section 4.2) on the noisy time series to decompose and assess the determinism rate for every IMF (see Section 4.2). As a result, two components were extracted: one stochastic and other deterministic. By individually analyzing the deterministic component, we noticed it behaves similarly to the sine function (see the second and the third curves in Figure 5.5(a)), what confirms our approach was capable of separating the deterministic and the stochastic components. Additionally, this component was reconstructed in the phase space (see Figure 5.5(b)), resulting in an attractor close to the expected one as presented in Figure 5.3(b). The distance threshold and the delay and embedded dimensions used to decompose the time series were $\varepsilon=0.001$, $\tau=1$, and $m=2$, respectively ${ }^{3}$. The delay and embedded parameters were also used to unfold the time series into the phase space.

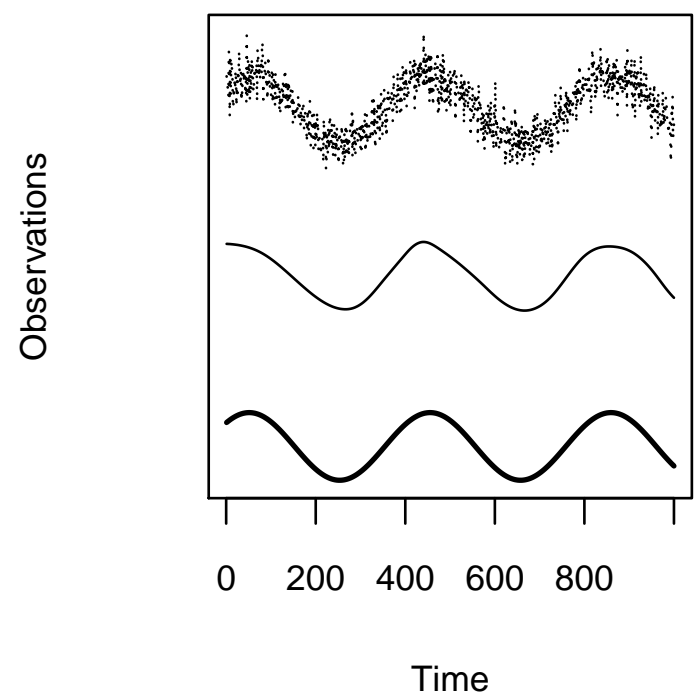

(a) Time series decomposition

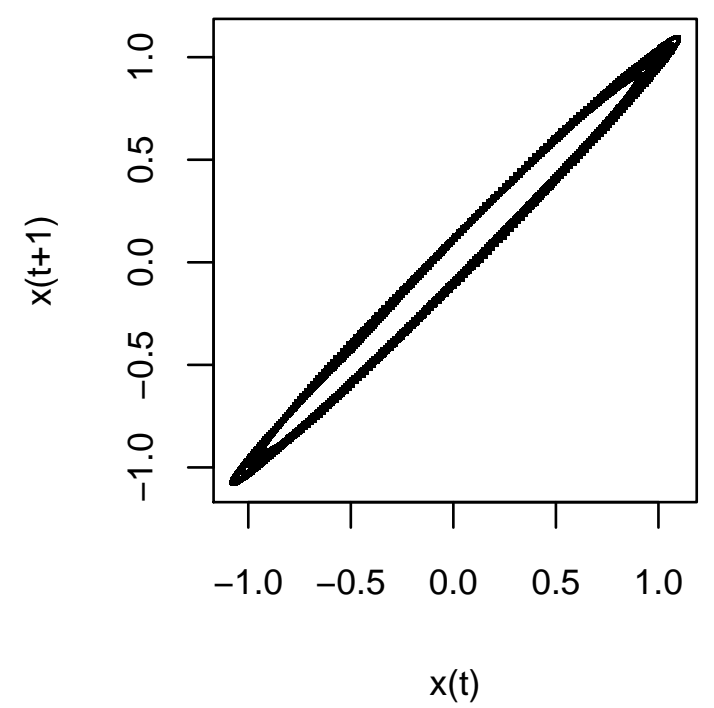

(b) Unfolded deterministic component

Figure 5.5: (a) The deterministic component (plot in the middle), obtained after decomposition, plotted against the undecomposed sinusoidal noisy time series (upper plot) and the expected behavior (lower plot). These series were shifted in $y$-axis in order to improve visualization. (b) The deterministic component reconstructed in the phase space.

After decomposing the noisy time series, we modeled the extracted components. As first step, the RBF and Polynomial models were used to model the deterministic component. The predicted observations for both models were also analyzed in phase space. According to Figure 5.6, we observe a similar behavior among the attractors produced by both models

\footnotetext{
${ }^{3}$ The delay and embedded dimensions were estimated using Average Mutual Information and False Nearest Neighbors methods. On the other hand, the distance threshold was estimated by empirical experimental analysis.
} 
and the expected attractor behavior (see Figures 5.3(b) and 5.5(b)).

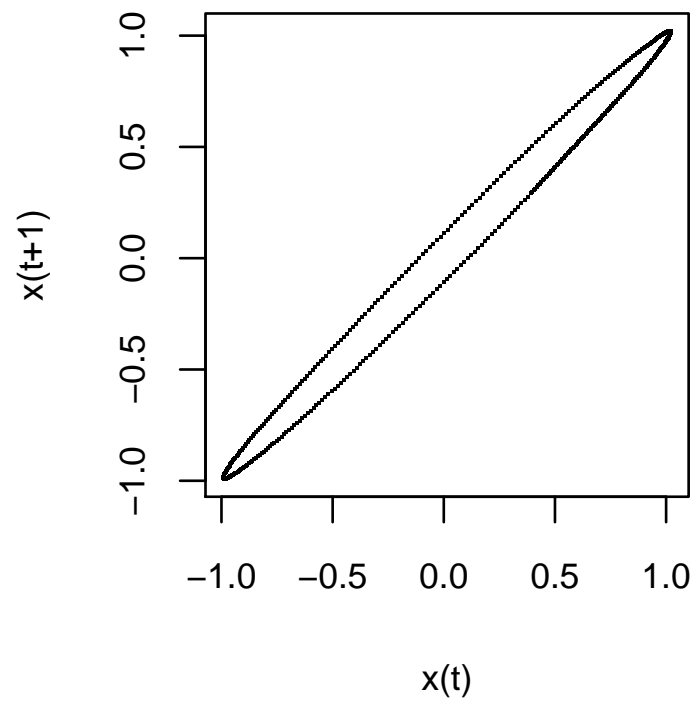

(a) Unfolded RBF prediction

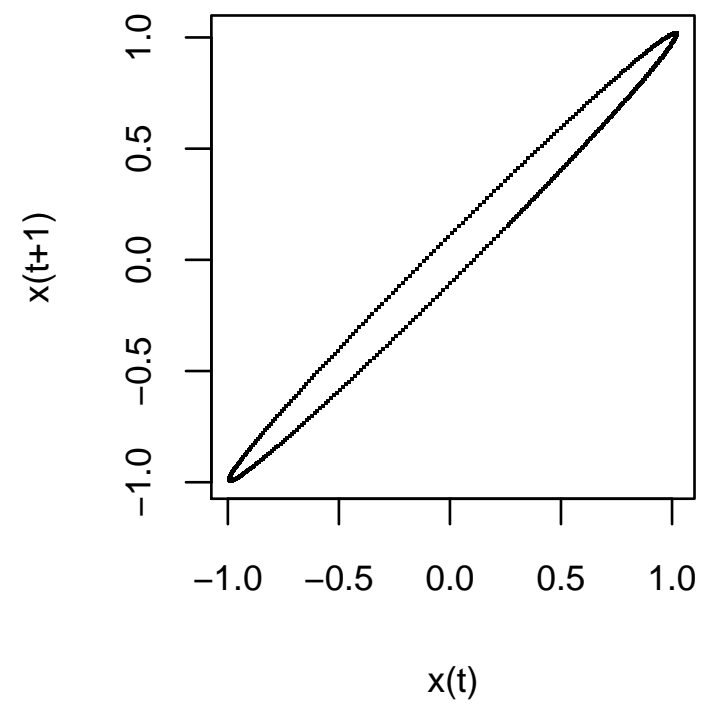

(b) Unfolded Polynomial prediction

Figure 5.6: (a) RBF-based predicted observations unfolded in phase space. (b) Polynomialbased predicted observations unfolded in phase space.

As next step, the decomposed stochastic component was modeled using an Autoregressive model. The observations predicted in this step were not graphically analyzed because they do not provide any meaningful information. Then, the accuracy of the three models was also analyzed using the MDDL measurement (see Section 5.3). Figures 5.7(a-c) show the MDDL plots comparing RBF, Polynomial, and AR models against the expected observations. Considering these figures, we observe Polynomial is the best model; In addition, the $\mathrm{RBF}$ also performed very well. On the other hand, the Autoregressive model resulted in the worst performance of all. These results are confirmed by MDDL scores: $M D D L_{R B F}=11.42$; $M D D L_{P O L}=9.04 ; M D D L_{A R}=25.19$, remembering lower MDDL scores are better.

Taking into account our hypothesis, in which we state it is important to analyze both components to model time series, we combined the deterministic and stochastic models to form a hybrid one. Consequently, two hybrid models were formed: i) RBF and AR models; and ii) Polynomial and AR models. Then, these two new hybrid models were used to predict observations, which were analyzed using MDDL. Figure 5.8 shows MDDL plots, indicating $\mathrm{RBF} / \mathrm{AR}$ and Polynomial/AR were better adjusted on the time series than each of them applied individually. This conclusion is also confirmed by MDDL scores: $M D D L_{R B F / A R}=$ 


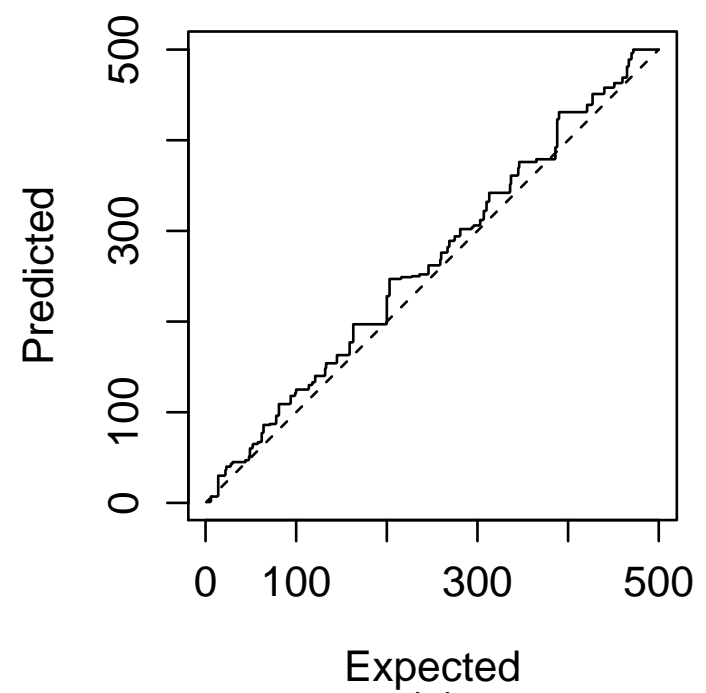

(a)

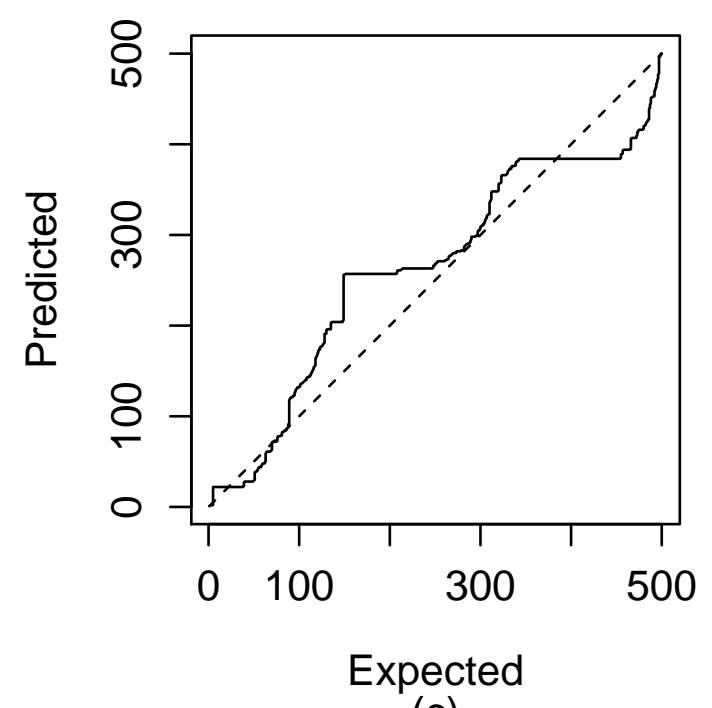

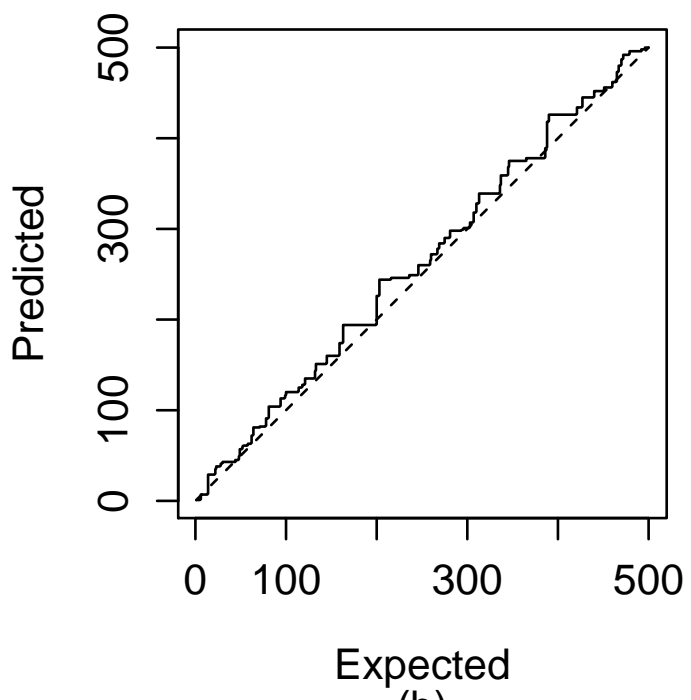

(b)

(c)

Figure 5.7: MDDL plot for the predicted and expected observations using (a) RBF, (b) Polynomial, and (c) AR models on the decomposed components.

11.02; $M D D L_{P O L / A R}=7.61$. Therefore, the best prediction results were obtained using the combination of Polynomial (deterministic) and Autoregressive (stochastic) models.

The last experiment in this scenario was executed without decomposing the time series. In this situation, we evaluated how good a model is when directly estimated on it. The MDDL results for this situation are illustrated in Figure 5.9, which is summed up by scores: $M D D L_{R B F}=120.78 ; M D D L_{P O L}=72.46 ; M D D L_{A R}=16.45$. These scores confirm 


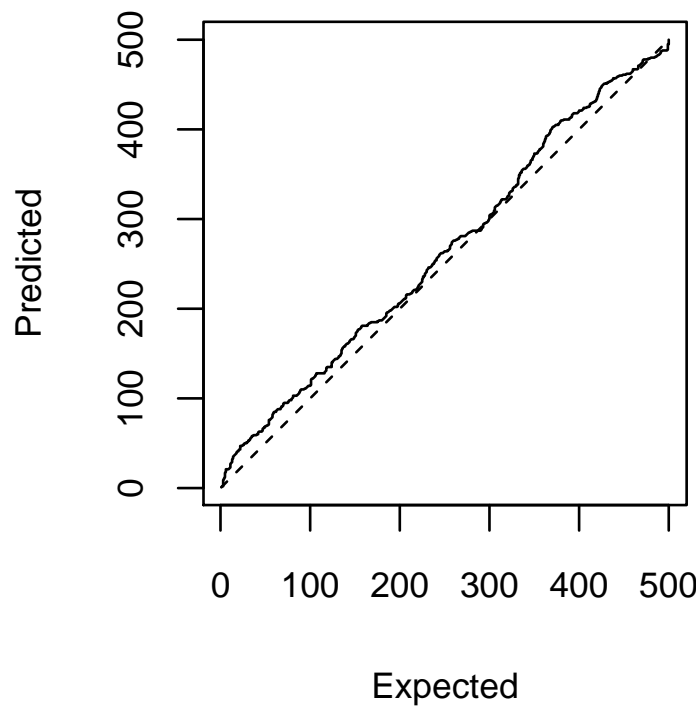

(a)

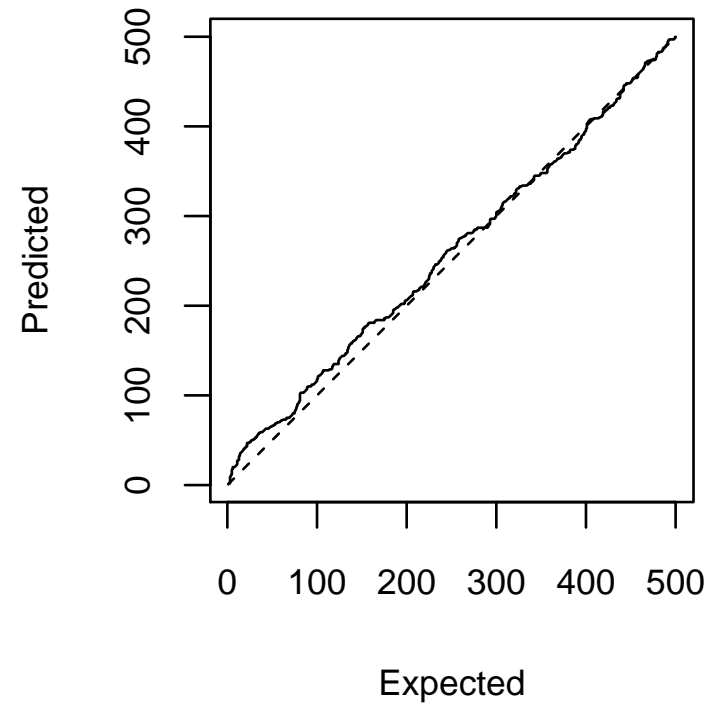

(b)

Figure 5.8: MDDL plot for the predicted and expected observations using the hybrid approaches: (a) RBF/AR, and (b) Polynomial/AR models.

the model adjusted on the undecomposed time series provided poor results because neither the stochastic nor the deterministic component was well estimated, what confirms the second part of our hypothesis.

Finally, Figures 5.10(a-c) present all predicted observations produced for every model previously discussed. By analyzing this figure we notice the best results for this scenario were obtained using hybrid models.

Similar experiments on different time series are presented in (Rios and Mello, 2013). In the next section, we present the results of additional experiments performed on different time series in order to evaluate the efficiency of our approach to improve time series modeling and prediction.

\subsection{Scenario II: Sine function plus ARMA and colored noise}

In this section, we assess the EMD-RP approach (see Section 4.2) using the same sine function of previous section, however adding two different stochastic components (Rios and Mello, 2013): i) an autoregressive moving average (ARMA) model with autoregressive order equals to 0.7 , moving-average order equals to -0.2 , and standard deviation equals to 0.25 ; and ii) a colored-noise process considering function $x(t)=0.5 x(t-1)+0.3 x(t-2)+0.1 x(t-$ 


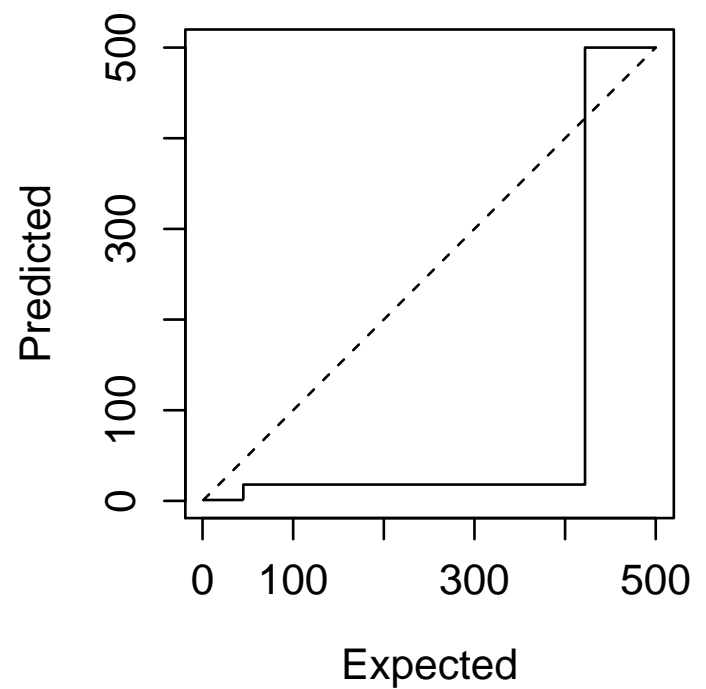

(a)

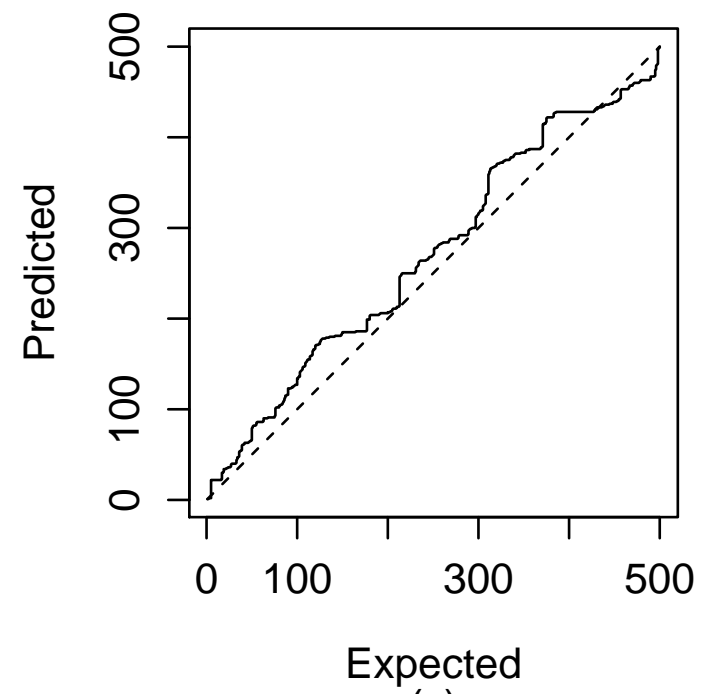

(c)

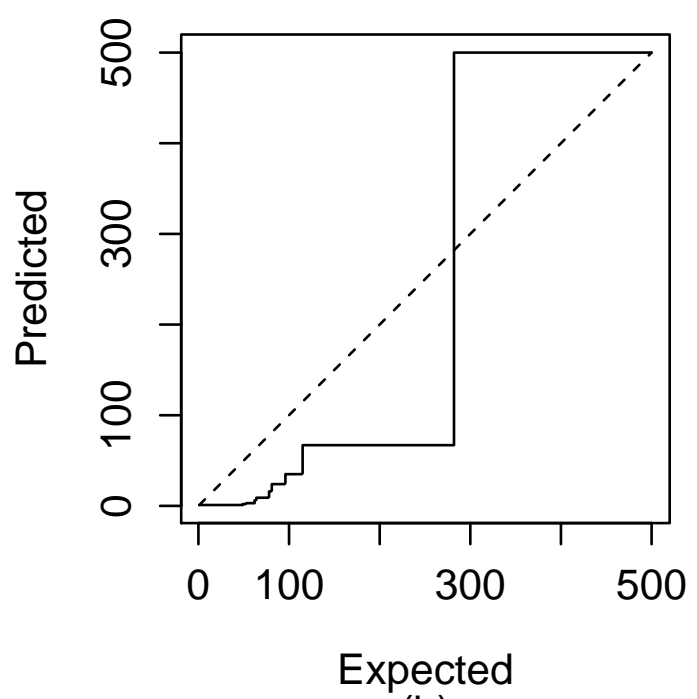

(b)

Figure 5.9: MDDL plot for the predicted and expected observations using RBF (a), Polynomial (b), and AR (c) models without decomposition.

3) $+\epsilon(\mu, \sigma)$, in which $\epsilon(\mu, \sigma)$ represents a Normal distribution with mean $\mu=0$ and standard deviation $\sigma=0.4$.

Hence, we started analyzing the first time series (Figure 5.11(a)) and its reconstruction in phase space (Figure 5.11(b)). After applying our approach, we extracted two components, one deterministic and another stochastic. In order to evaluate the decomposition approach, we also plotted the extracted deterministic component and its reconstructed attractor in 


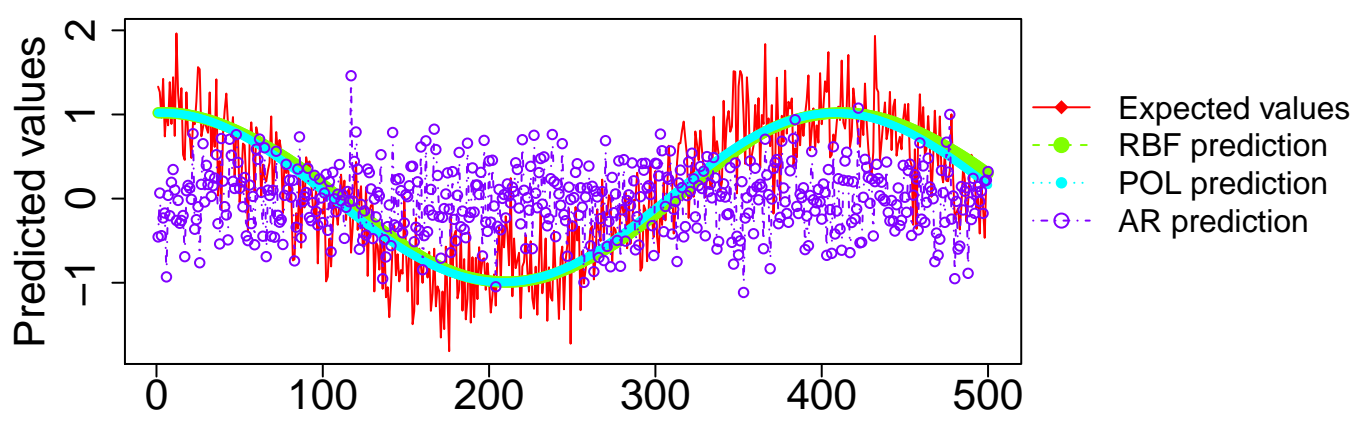

(a)

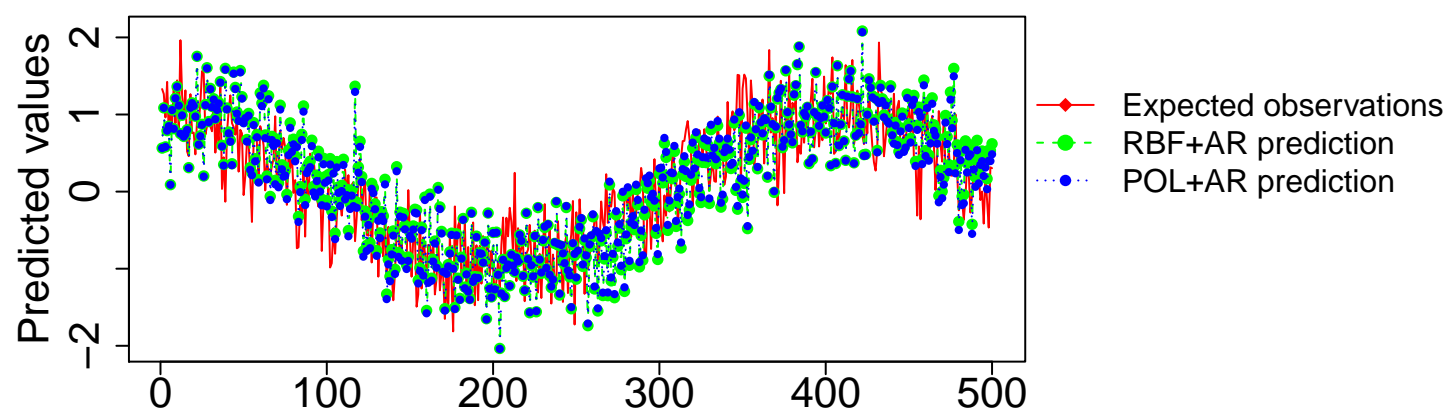

(b)

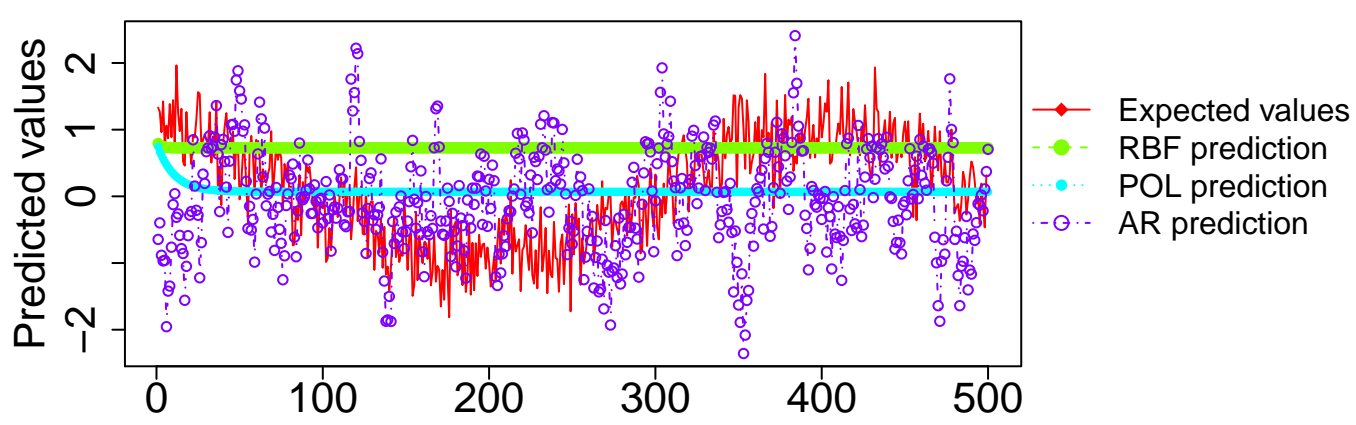

(c)

Figure 5.10: Comparison among the different prediction approaches: (a) RBF, Polynomial and AR models adjusted on the decomposed components; (b) hybrid models RBF/AR and Polynomial/AR adjusted on the decomposed components; (c) RBF, Polynomial and AR models directly applied on the undecomposed time series.

Figures 5.11(c) and 5.11(d), respectively. By analyzing all four figures, we observe the deterministic component obtained after decomposition has a similar behavior to the sine function (see Figure 5.3(b)) as expected, what confirms the decomposition step was capable of separating the deterministic component.

The same analyses performed on the time series presented in Scenario I (see Section 


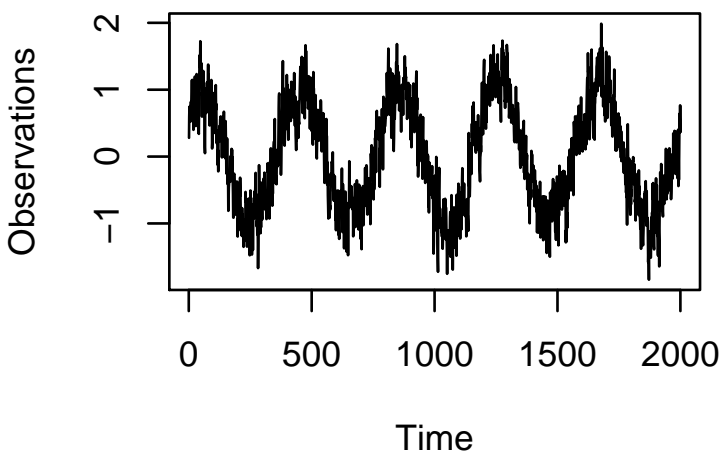

(a)

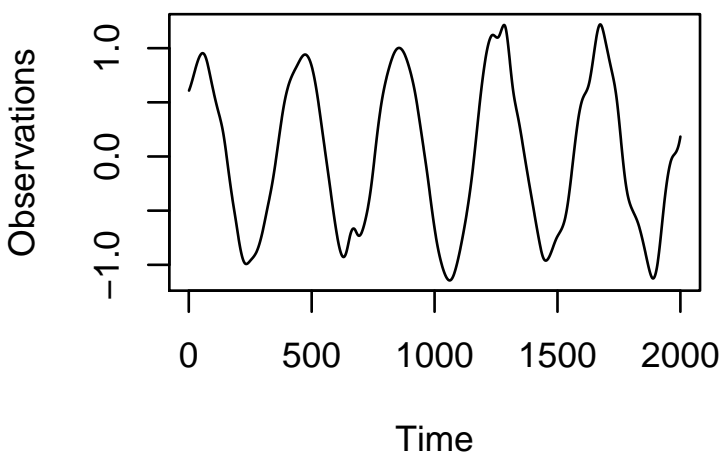

(c)

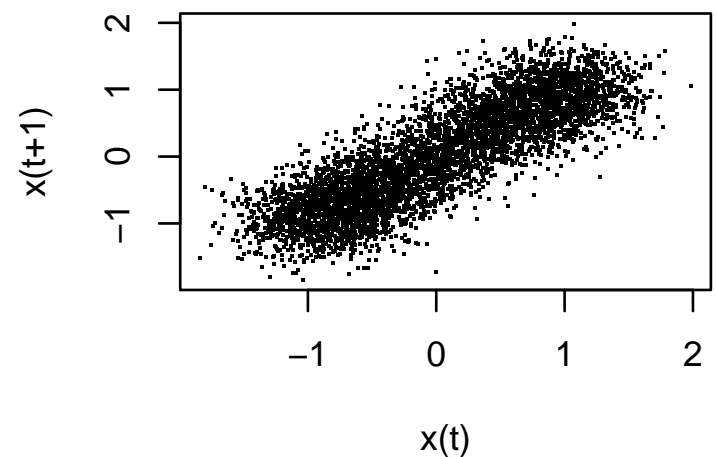

(b)

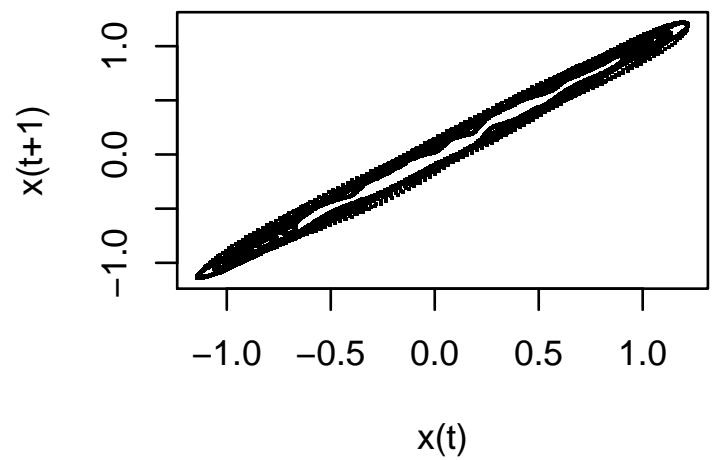

(d)

Figure 5.11: (a) Sine plus autoregressive moving average time series. (b) Sine plus autoregressive moving average time series unfolded in phase space. (c) The deterministic component. (d) The deterministic component unfolded in phase space.

5.4) were repeated on this time series, however, in this section, we summarized them in a single table. The resultant MDDL scores, presented in Table 5.1, were obtained in three steps (Rios and Mello, 2013). Firstly, models were adjusted on the original observations, i.e., without decomposition (rows RBF Undecomposed, Polynomial Undecomposed, and AR Undecomposed). Secondly, we analyzed models adjusted on the individual stochastic and deterministic components, named in Table 5.1 under rows RBF Decomposed, Polynomial Decomposed, and AR Decomposed. Finally, a third analysis was performed using hybrid models: i) RBF plus AR (row RBF Hybrid); and ii) Polynomial plus AR (Polynomial Hybrid). All models were evaluated considering MDDL scores. Besides scores, this table also shows the parameters used to adjust the RBF, Polynomial and AR models, which depend on models, i.e., for the AR model, it represents the Autoregressive order; for the RBF model, it represents the number of radial basis functions; and, for the Polynomial model, it corresponds to the Polynomial order. For all analyses, the distance threshold, the delay and 
embedded dimensions were set as follows $\varepsilon=0.005, \tau=1$ and $m=2$.

Table 5.1: Results considering models adjusted on the time series created by the combination of a sine function and an ARMA model.

\begin{tabular}{|lll|}
\hline \multicolumn{3}{|c|}{ ARMA noisy time series } \\
\hline & MDDL & Parameter \\
\hline AR Decomposed & 58.74 & 1 \\
AR Hybrid & - & - \\
AR Undecomposed & 32.27 & 1 \\
\hline & MDDL & Parameter \\
\hline POL Decomposed & 10.24 & 2 \\
POL Hybrid & $\mathbf{8 . 7 8}$ & $\mathbf{2}$ \\
POL Undecomposed & 52.1 & 2 \\
\hline & MDDL & Parameter \\
\hline RBF Decomposed & 29.31 & 200 \\
RBF Hybrid & 29.94 & 200 \\
RBF Undecomposed & 131.06 & 200 \\
\hline
\end{tabular}

According to results in Table 5.1, the best result was obtained using the hybrid model that combines the Polynomial and AR models, confirming our hypothesis as defined in the introduction.

Still considering the second scenario, we analyzed the time series created with the combination of the sine function and the colored-noise process (Rios and Mello, 2013). This time series and its reconstruction in phase space are shown in Figures 5.12(a) and 5.12(b), respectively. We also analyzed the deterministic component and its reconstructed attractor (Figures 5.12(c) and 5.12(d)). Considering all four figures, we again observe the deterministic component is very similar to the sine function as expected (see Figure 5.3(b)), evidencing our approach is capable of separating the deterministic component.

This time series was also analyzed considering the same steps previously presented and the results are summarized in Table 5.2 (Rios and Mello, 2013). For all analyses, the distance threshold, the delay and embedded dimensions were set as follows $\varepsilon=0.01, \tau=1$ and $m=2$.

By analyzing the MDDL scores, we observe the hybrid model Polynomial/AR outperformed the models directly adjusted on the undecomposed time series. Moreover, we notice AR Decomposed and AR Undecomposed models also presented good results what is explained by the strong stochastic influence on the resultant time series. In the next sec- 


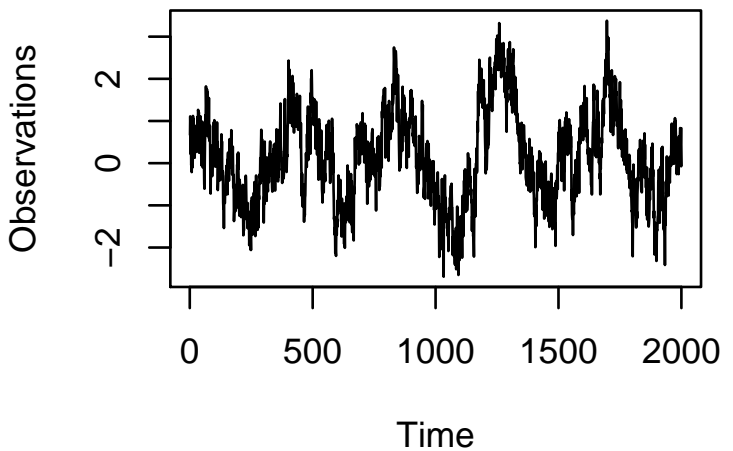

(a)

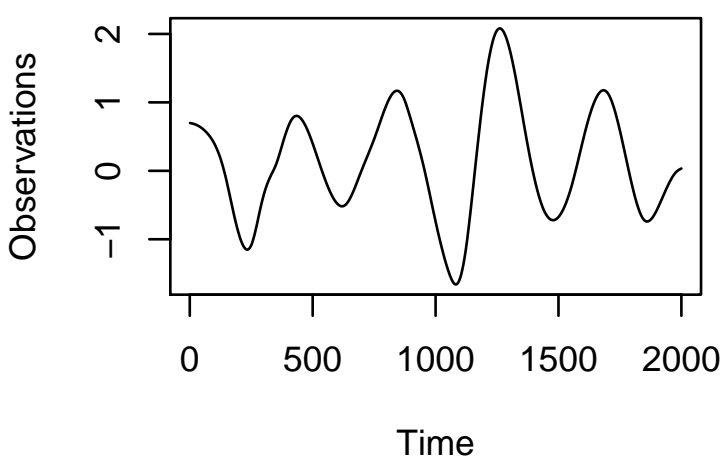

(c)

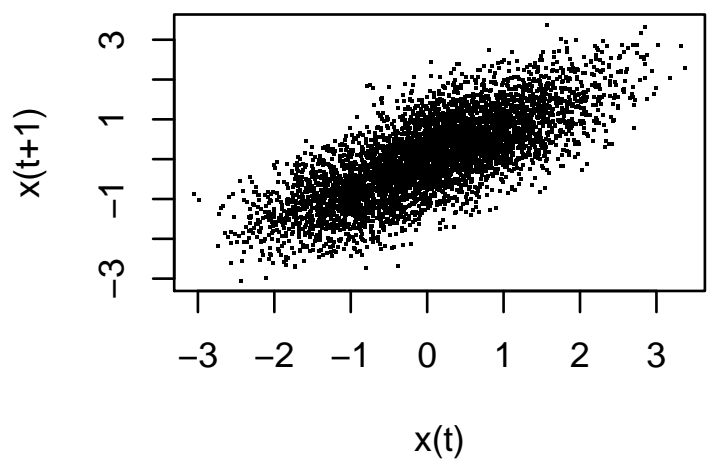

(b)

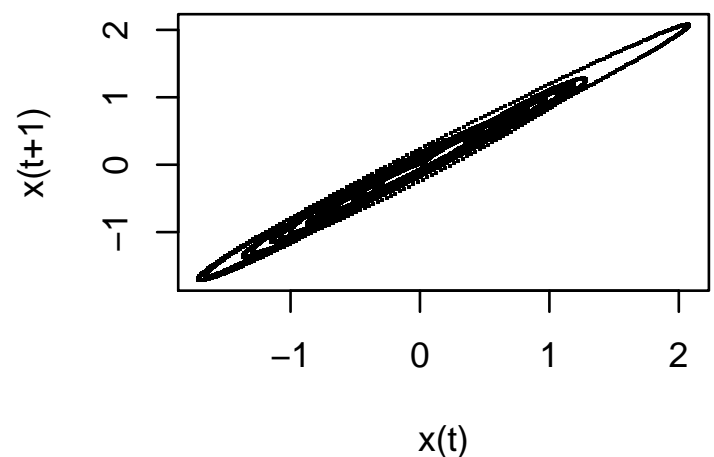

(d)

Figure 5.12: (a) Sine plus colored-noise time series. (b) Sine plus colored-noise time series unfolded in phase space. (c) The deterministic component. (d) The deterministic component unfolded in phase space.

Table 5.2: Results considering models adjusted on the time series created by the combination of a sine function and a colored noise.

\begin{tabular}{|lll|}
\hline \multicolumn{3}{|c|}{ Colored noisy time series } \\
\hline & MDDL & Parameter \\
\hline AR Decomposed & 24.81 & 1 \\
AR Hybrid & - & - \\
AR Undecomposed & 25.24 & 1 \\
\hline & MDDL & Parameter \\
\hline POL Decomposed & 24.30 & 2 \\
POL Hybrid & $\mathbf{1 5 . 3 6}$ & $\mathbf{2}$ \\
POL Undecomposed & 117.44 & 2 \\
\hline & MDDL & Parameter \\
\hline RBF Decomposed & 62.51 & 200 \\
RBF Hybrid & 37.49 & 200 \\
RBF Undecomposed & 80.42 & 200 \\
\hline
\end{tabular}

tion, we present results obtained by applying the EMD-RP approach on a set of ten time series combining the Lorenz system plus ten different white noise processes. 


\subsection{Scenario III: Lorenz system plus white noise}

In this third scenario we created ten time series combining the same deterministic component, i.e., the Lorenz system with parameters $\sigma=10, \rho=28$ and $\beta=8 / 3$, plus ten different white noise processes (stochastic components). White noise processes followed a Normal distribution with mean $\mu=0$ and ten different standard deviations: $\sigma \in\{0.05,0.1,0.15,0.20,0.25,0.3,0.35,0.4,0.45,0.5\}$. The additive noise of ten different standard deviations allowed to produced time series with different mixtures of deterministic and stochastic levels as seen in Figure 5.13.

The same analyses performed in Scenario I were repeated on this scenario and the results were summarized in Table 5.3. In order to simplify the understanding of this table, those ten time series were named as follows: Lorenz/WN0.05, Lorenz/WNO.1, ..., Lorenz/WNO.5. The distance threshold, the delay and embedded dimensions for every time series were set using Monte Carlo simulations. The main advantage of using Monte Carlos simulation is the possibility of evaluate a broad class of parameters (Kroese et al., $2011)$.

By analyzing this last table, we confirmed the modeling of stochastic and deterministic components outperforms models directly adjusted on undecomposed time series. In summary, the advantage of the decomposition approach was evident specially when considering time series Lorenz/WNO.15, Lorenz/WNO.2, Lorenz/WNO.25, Lorenz/WNO.3, Lorenz/WNO.35, Lorenz/WNO.4, Lorenz/WNO.45, and Lorenz/WNO.5.

\subsection{Concluding remarks}

The results obtained using the EMD-RP approach on different time series confirmed our hypothesis, which claims time series modeling is improved when considering stochastic and deterministic influences (Rios and Mello, 2013). In addition to the results and conclusions presented in this chapter, more experiments are presented in (Rios and Mello, 2013).

In order to understand these influences, we adjusted models on the decomposed components and evaluated their effectiveness through the prediction of observations and, in 
Table 5.3: Results obtained with the models ajusted on the noisy time series created for the third scenario, considering the same three analysis depicted in Tables 5.1 and 5.2.

\begin{tabular}{|llll|}
\hline \multicolumn{4}{c}{ Lorenz/WNO.05 } \\
\hline & MDDL & Threshold & Parameter \\
\hline AR Decomposed & 13.2098 & 0.1 & 1 \\
AR Hybrid & NA & NA & NA \\
AR Undecomposed & 28.7801 & 0.1 & 3 \\
\hline & MDDL & Threshold & Parameter \\
\hline POL Decomposed & 10.663 & 0.1 & 4 \\
POL Hybrid & 13.2463 & 0.1 & 2 \\
POL Undecomposed & 10.4365 & 0.1 & 6 \\
\hline & MDDL & Threshold & Parameter \\
\hline RBF Decomposed & 11.0025 & 0.1 & 9 \\
RBF Hybrid & 10.8586 & 0.1 & 9 \\
RBF Undecomposed & $\mathbf{7 . 7 0 8 1}$ & $\mathbf{0 . 1}$ & $\mathbf{9}$ \\
\hline & & & \\
\hline & Lorenz/WNO.1 & \\
\hline & MDDL & Threshold & Parameter \\
\hline AR Decomposed & 13.5726 & 0.5 & 1 \\
AR Hybrid & NA & NA & NA \\
AR Undecomposed & 29.8222 & 0.1 & 3 \\
\hline & MDDL & Threshold & Parameter \\
\hline POL Decomposed & 13.3481 & 0.5 & 4 \\
POL Hybrid & 12.9681 & 0.1 & 2 \\
POL Undecomposed & 14.4264 & 0.1 & 8 \\
\hline & MDDL & Threshold & Parameter \\
\hline RBF Decomposed & 11.1001 & 0.1 & 12 \\
RBF Hybrid & 13.4894 & 0.5 & 12 \\
RBF Undecomposed & $\mathbf{8 . 9 3 2 1}$ & $\mathbf{0 . 1}$ & $\mathbf{1 5 0}$ \\
\hline
\end{tabular}

\begin{tabular}{|llll|}
\hline \multicolumn{4}{l}{ Lorenz/WNO.3 } \\
\hline & MDDL & Threshold & Parameter \\
\hline AR Decomposed & 12.354 & 0.1 & 2 \\
AR Hybrid & NA & NA & NA \\
AR Undecomposed & 13.2887 & 0.1 & 1 \\
\hline & MDDL & Threshold & Parameter \\
\hline POL Decomposed & 10.5534 & 0.13 & 6 \\
POL Hybrid & $\mathbf{9 . 2 9 3 4}$ & $\mathbf{0 . 1 3}$ & $\mathbf{6}$ \\
POL Undecomposed & 12.4785 & 0.1 & 8 \\
\hline & MDDL & Threshold & Parameter \\
\hline RBF Decomposed & 14.3906 & 0.13 & 140 \\
RBF Hybrid & 12.4758 & 0.13 & 10 \\
RBF Undecomposed & 16.1188 & 0.1 & 140 \\
\hline
\end{tabular}

\begin{tabular}{|llll|}
\hline \multicolumn{4}{l}{ Lorenz/WNO.35 } \\
\hline & MDDL & Threshold & Parameter \\
\hline AR Decomposed & $\mathbf{1 0 . 4 6 6 2}$ & $\mathbf{0 . 1 2}$ & $\mathbf{1}$ \\
AR Hybrid & NA & NA & NA \\
AR Undecomposed & 16.2885 & 0.1 & 2 \\
\hline & MDDL & Threshold & Parameter \\
\hline POL Decomposed & 19.2775 & 0.12 & 4 \\
POL Hybrid & 11.2574 & 0.1 & 6 \\
POL Undecomposed & 25.3221 & 0.1 & 8 \\
\hline & MDDL & Threshold & Parameter \\
\hline RBF Decomposed & 14.4149 & 0.12 & 14 \\
RBF Hybrid & 11.7825 & 0.12 & 14 \\
RBF Undecomposed & 22.3546 & 0.1 & 150 \\
\hline
\end{tabular}

\begin{tabular}{|llll|}
\hline \multicolumn{4}{c}{ Lorenz/WNO.15 } \\
\hline & MDDL & Threshold & Parameter \\
\hline AR Decomposed & 27.3868 & 0.1 & 3 \\
AR Hybrid & NA & NA & NA \\
AR Undecomposed & 30.2871 & 0.1 & 3 \\
\hline & MDDL & Threshold & Parameter \\
\hline POL Decomposed & $\mathbf{1 2 . 2 5 8}$ & $\mathbf{0 . 1}$ & $\mathbf{6}$ \\
POL Hybrid & 12.9851 & 0.11 & 4 \\
POL Undecomposed & 14.0396 & 0.1 & 4 \\
\hline & MDDL & Threshold & Parameter \\
\hline RBF Decomposed & 14.5949 & 0.1 & 14 \\
RBF Hybrid & 13.0927 & 0.1 & 14 \\
RBF Undecomposed & 15.713 & 0.1 & 150 \\
\hline
\end{tabular}

\begin{tabular}{|llll|}
\hline \multicolumn{4}{c|}{ Lorenz/WNO.4 } \\
\hline & MDDL & Threshold & Parameter \\
\hline AR Decomposed & 14.5615 & 0.19 & 1 \\
AR Hybrid & NA & NA & NA \\
AR Undecomposed & 14.4032 & 0.1 & 1 \\
\hline & MDDL & Threshold & Parameter \\
\hline POL Decomposed & $\mathbf{1 2 . 4 9 4 4}$ & $\mathbf{0 . 1 9}$ & $\mathbf{4}$ \\
POL Hybrid & 13.6556 & 0.1 & 6 \\
POL Undecomposed & 22.0838 & 0.1 & 8 \\
\hline & MDDL & Threshold & Parameter \\
\hline RBF Decomposed & 15.2845 & 0.1 & 12 \\
RBF Hybrid & 13.0668 & 0.1 & 160 \\
RBF Undecomposed & 85.9424 & 0.1 & 10 \\
\hline
\end{tabular}

\begin{tabular}{|llll|}
\hline \multicolumn{4}{c}{ Lorenz/WNO.2 } \\
\hline & MDDL & Threshold & Parameter \\
\hline AR Decomposed & 12.9019 & 0.1 & 3 \\
AR Hybrid & NA & NA & NA \\
AR Undecomposed & 28.6052 & 0.1 & 1 \\
\hline & MDDL & Threshold & Parameter \\
\hline POL Decomposed & 14.7866 & 0.13 & 4 \\
POL Hybrid & 14.5328 & 0.13 & 2 \\
POL Undecomposed & 12.9234 & 0.1 & 4 \\
\hline & MDDL & Threshold & Parameter \\
\hline RBF Decomposed & 10.3001 & 0.13 & 10 \\
RBF Hybrid & $\mathbf{8 . 8 5 5 9}$ & $\mathbf{0 . 1 3}$ & $\mathbf{1 0}$ \\
RBF Undecomposed & 13.4848 & 0.1 & 10 \\
\hline
\end{tabular}

\begin{tabular}{|llll|}
\hline \multicolumn{4}{l}{ Lorenz/WNO.45 } \\
\hline & MDDL & Threshold & Parameter \\
\hline AR Decomposed & 11.8754 & 0.1 & 1 \\
AR Hybrid & NA & NA & NA \\
AR Undecomposed & 14.5827 & 0.1 & 1 \\
\hline & MDDL & Threshold & Parameter \\
\hline POL Decomposed & 18.7545 & 0.18 & 4 \\
POL Hybrid & 13.9668 & 0.18 & 2 \\
POL Undecomposed & 93.0358 & 0.1 & 4 \\
\hline & MDDL & Threshold & Parameter \\
\hline RBF Decomposed & $\mathbf{1 1 . 3 1 9 2}$ & $\mathbf{0 . 1 8}$ & $\mathbf{1 0}$ \\
RBF Hybrid & 13.1132 & 0.1 & 160 \\
RBF Undecomposed & 84.7882 & 0.1 & 9 \\
\hline
\end{tabular}

\begin{tabular}{|llll|}
\hline \multicolumn{4}{c}{ Lorenz/WNO.25 } \\
\hline & MDDL & Threshold & Parameter \\
\hline AR Decomposed & 12.9715 & 0.1 & 3 \\
AR Hybrid & NA & NA & NA \\
AR Undecomposed & 13.5808 & 0.1 & 1 \\
\hline & MDDL & Threshold & Parameter \\
\hline POL Decomposed & 13.5721 & 0.11 & 8 \\
POL Hybrid & 15.0262 & 0.11 & 2 \\
POL Undecomposed & 12.9866 & 0.1 & 8 \\
\hline & MDDL & Threshold & Parameter \\
\hline RBF Decomposed & 10.5117 & 0.11 & 140 \\
RBF Hybrid & $\mathbf{9 . 7 9 0 9}$ & $\mathbf{0 . 1}$ & $\mathbf{9}$ \\
RBF Undecomposed & 13.1786 & 0.1 & 140 \\
\hline
\end{tabular}

\begin{tabular}{|llll|}
\hline \multicolumn{4}{l}{ Lorenz/WNO.5 } \\
\hline & MDDL & Threshold & Parameter \\
\hline AR Decomposed & 13.1155 & 0.11 & 1 \\
AR Hybrid & NA & NA & NA \\
AR Undecomposed & 13.7871 & 0.1 & 2 \\
\hline & MDDL & Threshold & Parameter \\
\hline POL Decomposed & 20.656 & 0.11 & 6 \\
POL Hybrid & 11.5261 & 0.11 & 4 \\
POL Undecomposed & 101.4279 & 0.1 & 8 \\
\hline & MDDL & Threshold & Parameter \\
\hline RBF Decomposed & 13.0818 & 0.11 & 10 \\
RBF Hybrid & $\mathbf{1 0 . 4 0 7 1}$ & $\mathbf{0 . 1 1}$ & $\mathbf{1 6 0}$ \\
RBF Undecomposed & $\mathbf{7 7 . 2 0 3 2}$ & 0.1 & 10 \\
\hline
\end{tabular}




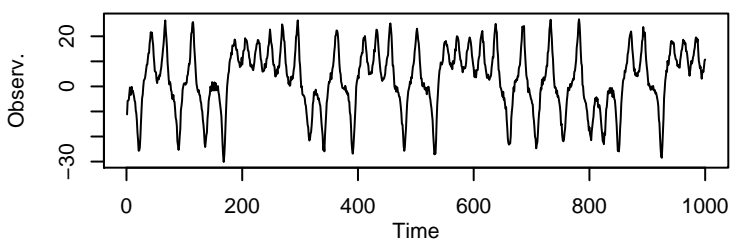

(a) Lorenz system + white noise $(\sigma=0.05)$

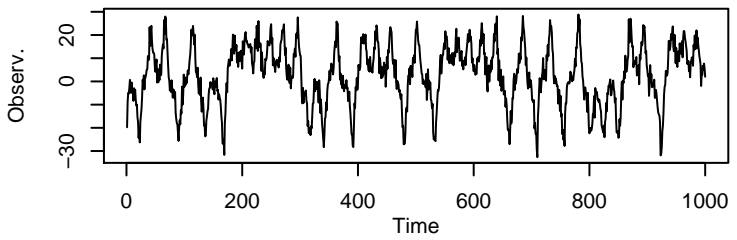

(c) Lorenz system + white noise $(\sigma=0.15)$

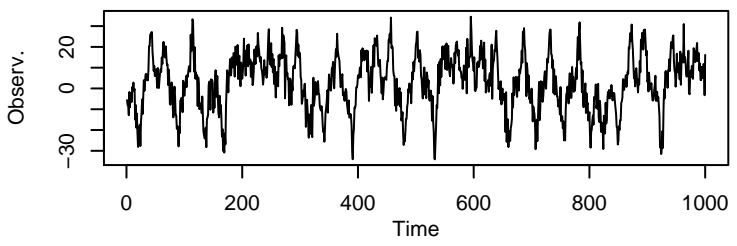

(e) Lorenz system + white noise $(\sigma=0.25)$

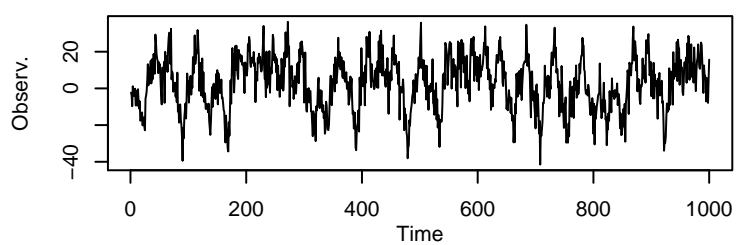

(g) Lorenz system + white noise $(\sigma=0.35)$

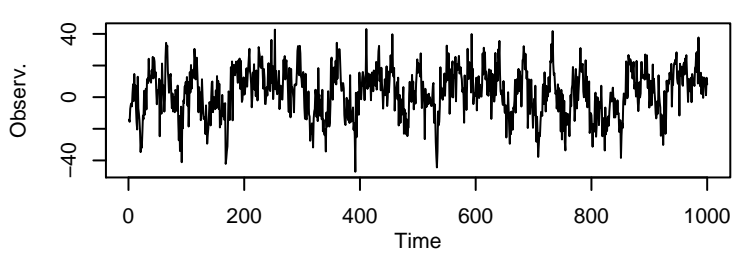

(i) Lorenz system + white noise $(\sigma=0.45)$

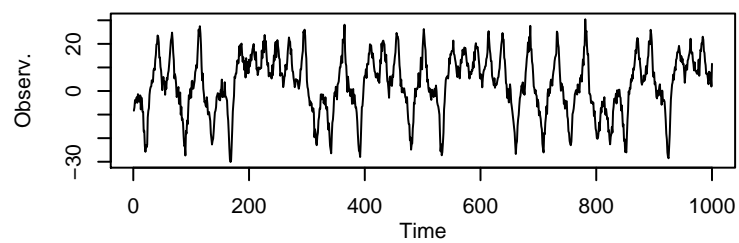

(b) Lorenz system + white noise $(\sigma=0.1)$

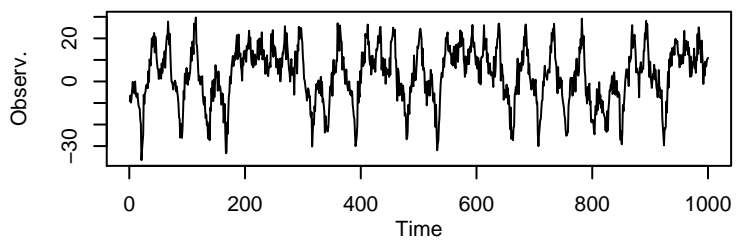

(d) Lorenz system + white noise $(\sigma=0.2)$

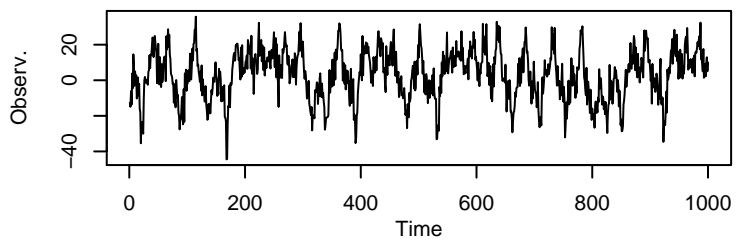

(f) Lorenz system + white noise $(\sigma=0.3)$

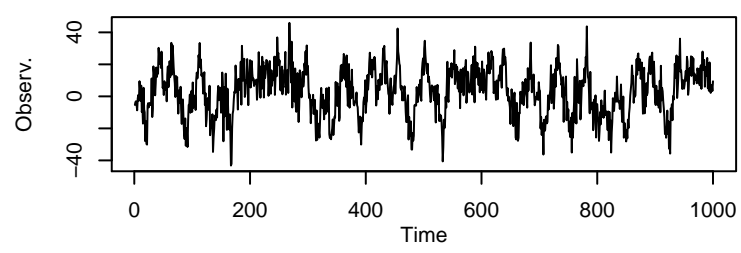

(h) Lorenz system + white noise $(\sigma=0.4)$

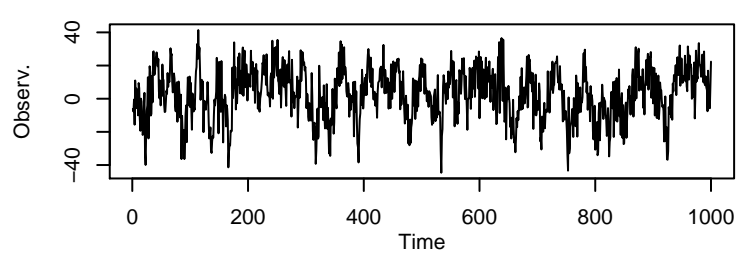

(j) Lorenz system + white noise $(\sigma=0.5)$

Figure 5.13: Lorenz time series influenced by different stochastic components.

that manner, we analyzed how well models perform under unknown situations. Results confirmed the importance of the decomposition step and the individual modeling of the deterministic and stochastic components (Rios and Mello, 2013).

Even though the application of our decomposition approach was advantageous in most of experiments, there are situations in which the stochastic component has little influence (basically low magnitude), consequently we can simply consider the deterministic component (Rios and Mello, 2013). For example, this situation is verified in time series 
Lorenz/WNO.05 and Lorenz/WNO.1 (see Table 5.3). On the other hand, when time series are strongly influenced by the stochastic component, the deterministic one is useless. In both situations, the modeling of the undecomposed time series provides better results. However, we emphasize this is not a problem with our decomposition approach, but a general issue faced by the area of time series analysis (Rios and Mello, 2013). 
CHAPTER

\section{Real-World Application}

\subsection{Introduction}

In this chapter, we demonstrate the importance of decomposing time series in a realworld application. However, instead of predicting observations, the decomposed components were analyzed in order to detect patterns and extract information embedded in time series. The experiments presented in this chapter were also important to confirm the first part of our hypothesis, what was formally analyzed in Chapter 3.

The analysis of this real-world application was performed in collaboration with Prof. Lael Parrott and Prof. Holger Lange during a visit to the Université de Montréal - Canada ${ }^{1}$. In summary, Prof. Parrott leads the Complex Environmental Systems Laboratory in the Department of Earth \& Environmental Sciences and Biology at the University of British Columbia (Canada) and Prof. Lange is with the Norwegian Forest and Landscape Institute (Norway).

The analyzed application is based upon the remote sensing of the Earth surface. This

${ }^{1}$ This work was supported by CAPES (Coordination for Enhancement of Higher Education Personnel) and FAPESP (São Paulo Research Foundation) under grant numbers 5983-11-8 and 2009/18293-9. 
application has become an important tool to provide data about global changes and the impact of human actions, such as deforestation and cropland expansion, on the environment (Rowhani et al., 2011; Lepers et al., 2005). In order to perform a proper analysis, one may periodically collect as much data as possible from different systems, such as temperature, rainfall, and radiation absorption on different World locations.

The analysis of these data is not an easy task due to the amount of collected observations and the complex relationships among different systems driving the dynamics of the observed variables. Therefore, the use of an approach to extract information from temporal geospatial data, organized as time series, and correlate implicit patterns among different systems is an important requirement.

The amount of collected observations, however, represents a limitation for the EMD-RP approach (see Section 4.2), once it requires supervision to define some parameters. This motivated us to design our second approach, referred to as EMD-MI (see Section 4.3), which combines the Empirical Mode Decomposition (EMD) and Mutual Information (MI) methods. According to experimental analyses, we confirmed this second approach extracts stochastic and deterministic components similarly to the EMD-RP approach, regardless parametrization supervision.

Another difference among the results presented in Chapter 5 and in this chapter is the measurement used to evaluate the experiments. The MDDL measurement is not used here, once the decomposed components are not used to predict observations. Instead, we considered the Determinism Rate presented in Section 4.3.2. This measure permitted correlating time series with different dynamics and, consequently, to better understanding their behaviors. In the next section, we present the real-world application in details.

\subsection{Experimental evaluation}

The effectiveness of the EMD-MI approach was evaluated using real-world environmental data. The datasets used for this purpose were the Fraction of Absorbed Photosynthesis Active Radiation (FAPAR) (Gobron et al., 2000; Mahecha et al., 2010), Rainfall (Rienecker et al., 2012; Mahecha et al., 2010), and Temperature (Rienecker et al., 2012; Mahecha et al., 2010). 
FAPAR $^{2}$ is one of the Land Surface products provided by the Institute for Environment and Sustainability (IES) of the European Commission's Joint Research Centre (JRC). The unit responsible to manage this dataset is the Global Environment Monitoring (GEM) unit, which is responsible to release products and services derived from the analysis of satellite remote sensing data. The FAPAR observations are collected from the Moderate Resolution Imaging Spectroradiometer (MODIS) remote sensor, located on board the National Aeronautics and Space Administration (NASA) Terra and Aqua satellite platforms.

In summary, this dataset contains the fraction of the incoming solar radiation absorbed by plants and other photosynthetic organisms on the Earth surface (Gobron et al., 2000; Fensholt et al., 2004; Gobron et al., 2010a,b; Mahecha et al., 2010). In our experiments, we used the FAPAR global scale dataset, which was monthly monitored from September 1997 to June 2006. These monitoring results are represented as a 2D World map spatially distributed over a matrix with 1080 rows and 2160 columns, in a $0.5^{\circ} \times 0.5^{\circ}$ resolution $(3 \times 3$ pixels), corresponding to an area of approximately $6 \mathrm{~km}^{2}$.

The monitoring accuracy depends on some natural aspects, like the presence of clouds between the remote sensor and the Earth surface. In order to overcome this drawback, three observations are used to represent a month and every observation is a mean of ten days. Thus, the dataset considered in this work contains 106 monitored months what ended up producing a time series with 318 observations for every location in the 2D World map. Figure 6.1 shows six different 2D World maps in which every pixel represents one observation of a time series of FAPAR dataset, consequently, the each map represents a different instant over time.

The second dataset used to evaluate our approach is provided by the Global Precipitation Climatology Centre (GPCC) ${ }^{3}$ (Rienecker et al., 2012). This dataset, here referred to as Rainfall, contains information about precipitations monthly collected on the Earth surface in a $0.5^{\circ} \times 0.5^{\circ}$ resolution. The collected values are spatially distributed over a $2 \mathrm{D}$ World map, represented as a matrix with 292 rows and 720 columns. Every point (cell) in this matrix is composed of a time series with 288 observations.

The last dataset used in our evaluation process represents the global temperature (Rie-

\footnotetext{
${ }^{2}$ Further details about the dataset and data processing are available at http://fapar.jrc.ec.europa.eu/ Home.php.

${ }^{3}$ Further details about the dataset and data processing are available at http://www.esrl.noaa.gov/psd/ data/gridded/data.gpcc.html\#detail
} 

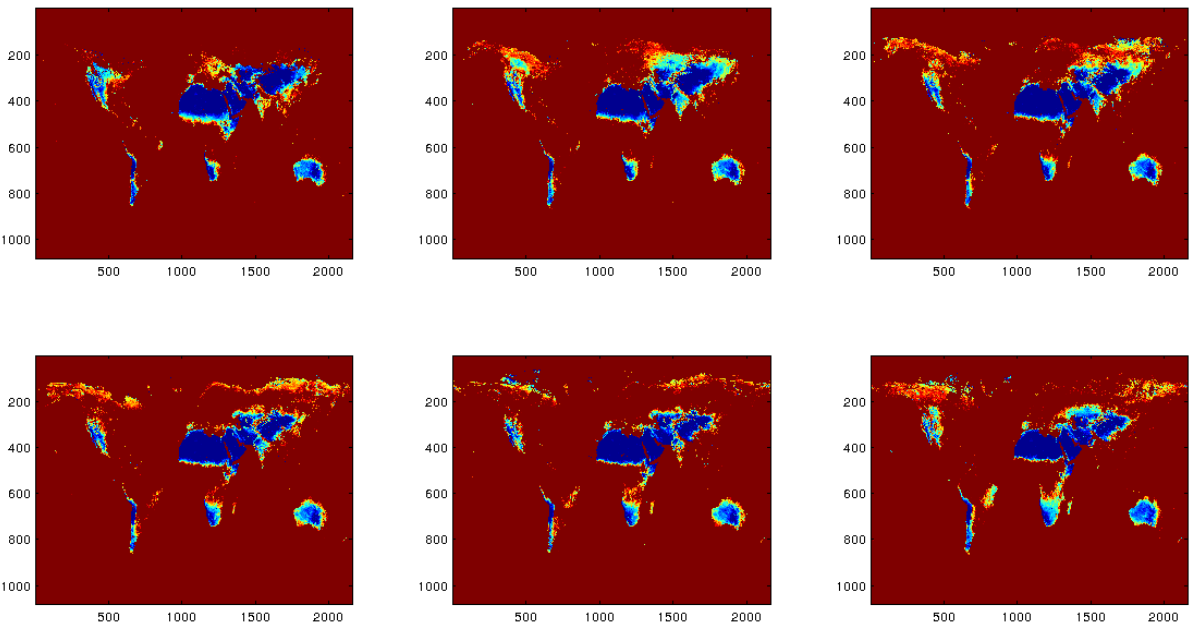

Figure 6.1: $2 \mathrm{D}$ World maps to illustrate the FAPAR dataset.
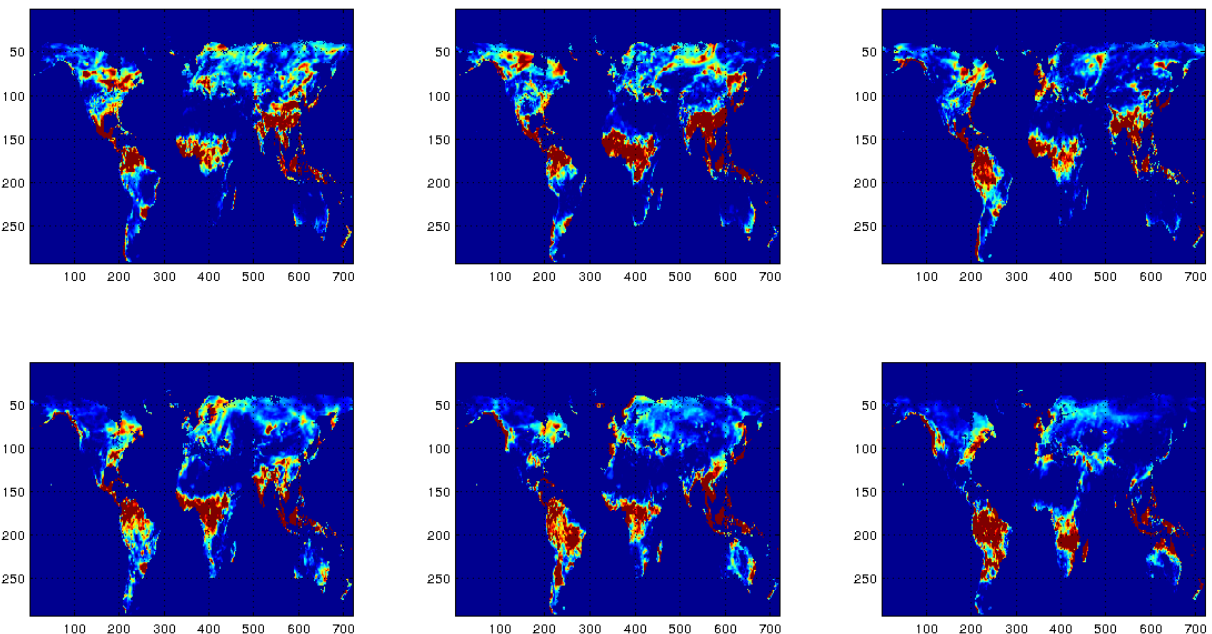

Figure 6.2: Six 2D World maps to illustrate the Rainfall dataset.

necker et al., 2012), which was monthly monitored on the Earth surface in a $0.5^{\circ} \mathrm{x} 0.5^{\circ}$ resolution. The values monitored for this dataset are spatially distributed over a $2 \mathrm{D}$ World map, in which every point is composed of a time series with 504 observations.

The FAPAR dataset is composed of 2,332, 800 time series, resulting in $741,830,400$ observations. On the other hand, the Rainfall dataset has 60,549, 120 observations organized in 210, 240 time series. Finally, the Temperature dataset has 51,912 time series with a total of 26, 163, 648 observations. The huge amount of data makes difficult to, individually, estimate 

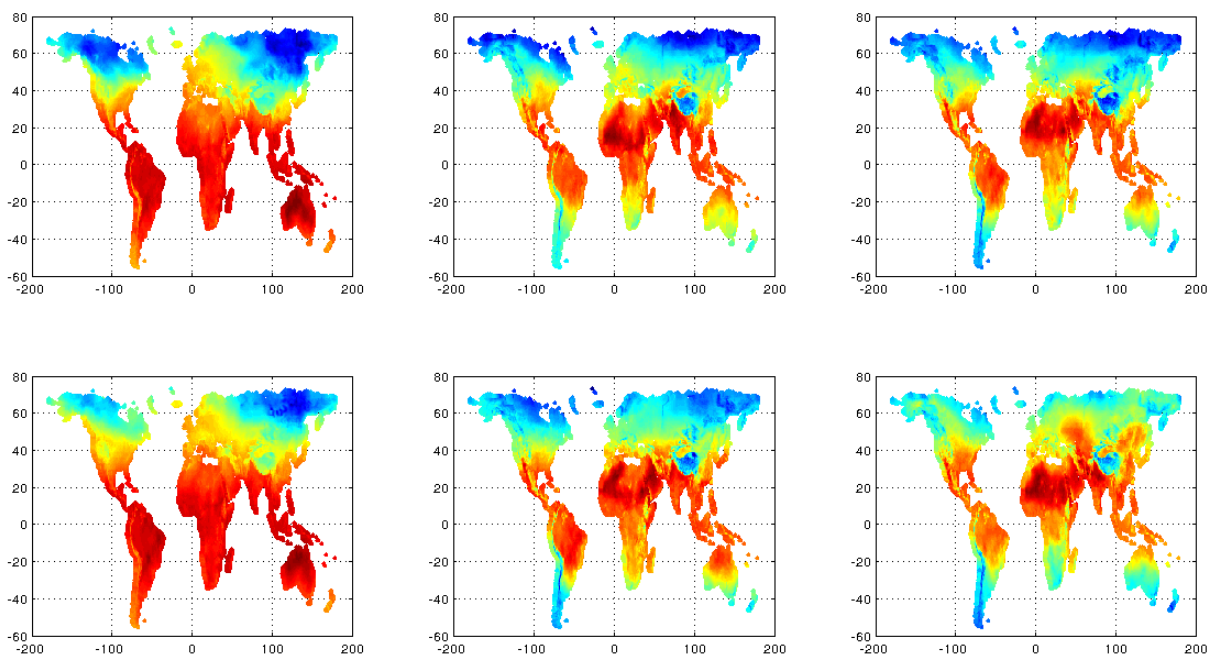

Figure 6.3: Six 2D World maps to illustrate the Temperature dataset.

and evaluate the EMD-RP parameters. This drawback motivated us to design EMD-MI, which is an unsupervised and nonparametric approach.

\subsection{Results}

In this section, we present the results obtained by applying the EMD-MI approach on all three datasets.

\subsubsection{Dataset: FAPAR}

The application of EMD-MI in the entire FAPAR dataset produced a new 2D World map (Figure 6.4), in which the percentage of deterministic influences correspond to gradient colors, i.e., the most stochastic time series $\left(R_{\operatorname{det}}(\cdot) \approx 0\right)$ are in blue, whereas the most deterministic ones $\left(R_{\operatorname{det}}(\cdot) \approx 1\right)$ are in red.

The first conclusion we draw considering this 2D World map is related to determinism rates present in the FAPAR dataset. There are two deterministic patterns: i) the first one is related to the total reflection of radiation on specific Earth regions. This situation happens when the surface is covered by water, ice, or desert sands, for example. In such places trees and leaves are sparse or absent; ii) the second deterministic type happens when the analyzed region is covered by plants that absorb almost all radiation emitted by the sun, 


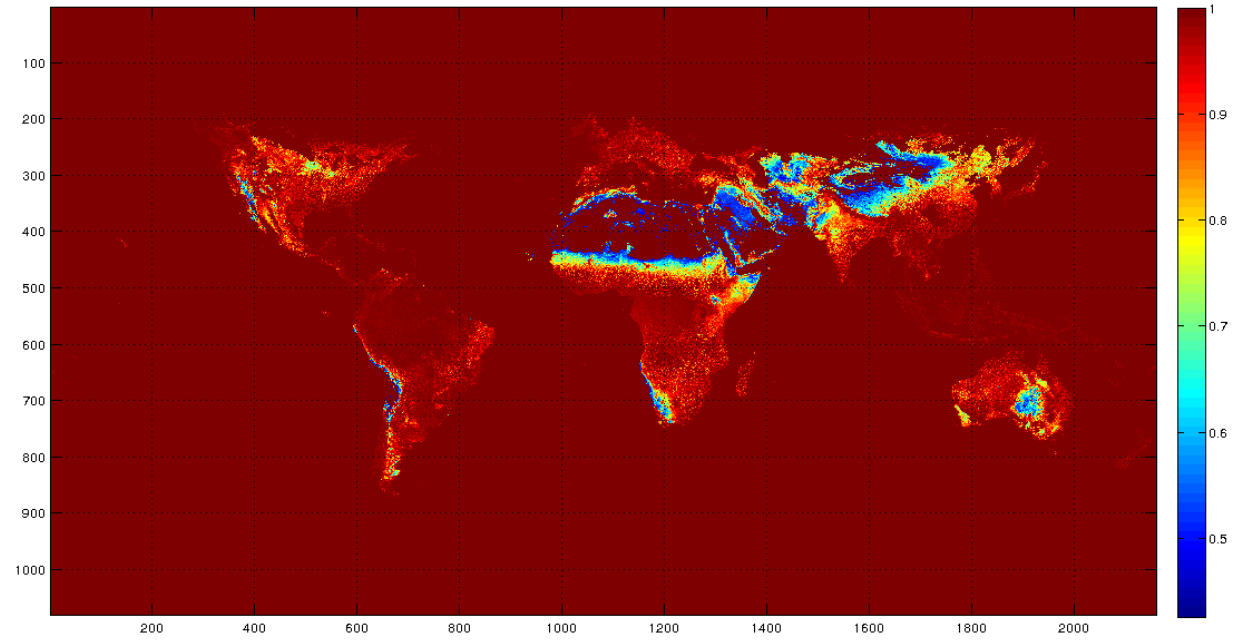

Figure 6.4: Determinism map obtained with EMD-MI to estimate determinism rates on FAPAR dataset.

e.g., in tropical rainforests. In both situations, the determinism rate is close to one, i.e., the surface will totally either absorb or reflect the radiation.

On the other hand, there are seven regions, whose the determinism rate is low. These regions are located at: 1) the west side of North America; 2) the west side of South America; 3) the North Africa; 4) the west side of South Africa; 5) the Middle East; 6) the central region of Asia; and 7) the central region of Australia. We observe all of these regions are characterized by semiarid vegetation under highly variable rainfall regimes.

\subsubsection{Dataset: Rainfall}

The results after analyzing the rainfall dataset are presented in Figure 6.5. By looking at this map, we observe that fully deterministic time series are less frequent in this dataset than in FAPAR, in addition regions with high determinism levels are common in areas of tropical rain forests. In addition to this pattern, we also notice that high stochastic levels occur in similar regions for FAPAR and Rainfall World maps.

In order to make evident similarities, we created two binary World maps (Figures 6.6(a) and 6.6(b)) based on the analysis of the deterministic maps generated for FAPAR and Rainfall datasets (Figures 6.4 and 6.5). These maps were obtained considering the heaviside step function presented in Equation 6.1. The threshold $\delta$ was set to 0.55 based on an 


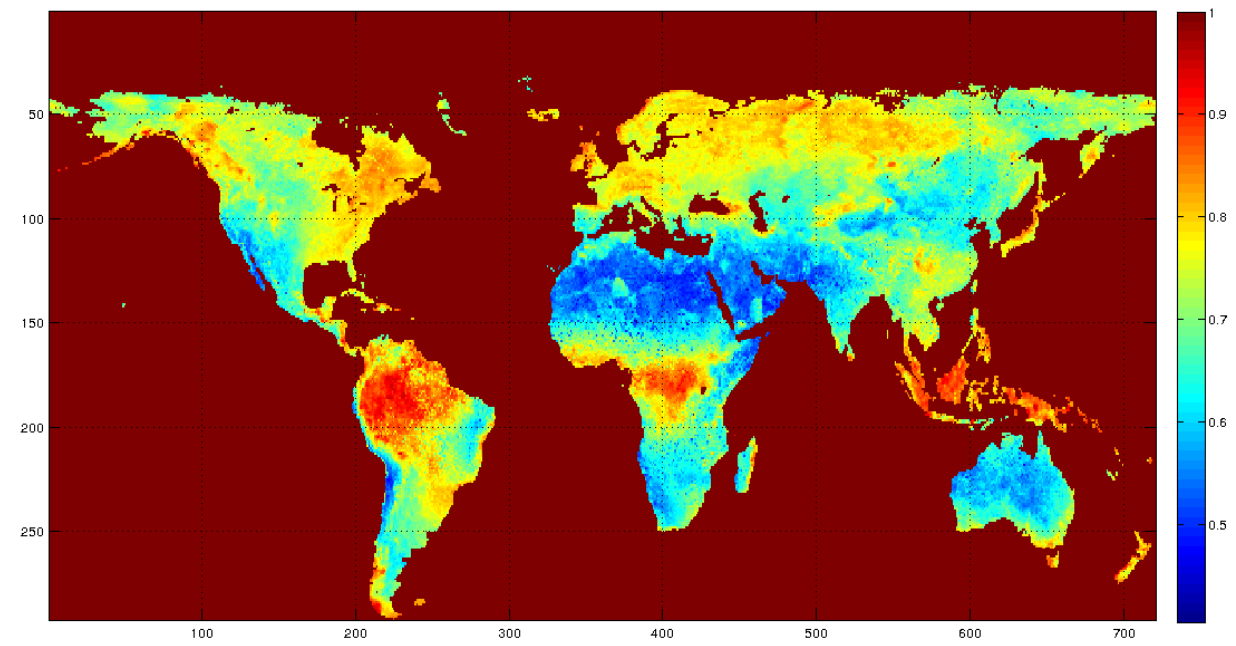

Figure 6.5: Deterministic 2D World map obtained with EMD-MI on Rainfall dataset.

empirical analysis, which allowed to emphasize the stochasticity in both maps.

$$
\hat{R}_{\operatorname{det}}(t)= \begin{cases}0: & R_{\operatorname{det}}(t)<\delta \\ 1: & R_{\operatorname{det}}(t) \geq \delta\end{cases}
$$

\subsubsection{Dataset: Temperature}

The results obtained by analyzing the temperature dataset are presented in Figure 6.7. By looking at this figure, we observe that deterministic time series are approximately located in between latitudes $45^{\circ} \mathrm{N}$ and $45^{\circ} \mathrm{S}$.

Aiming at better understanding the low determinism rate obtained for the region located at the north of latitude $45^{\circ} \mathrm{N}$, it is important to know the influences of air masses around the planet. Those masses are volumes of air that influence the weather over the world. An air mass is defined according to the presence of temperature and moisture. Moist air masses are originated on the sea, whereas dry ones are formed on continents. Similarly, the temperature influences the air masses in two different ways. Air masses originated from tropical regions are warmer and polar regions form colder masses.

The notation used to classify air masses is normally represented by two letters. The first letter, used in lowercase, is related to the moisture. Masses formed on the continent and 


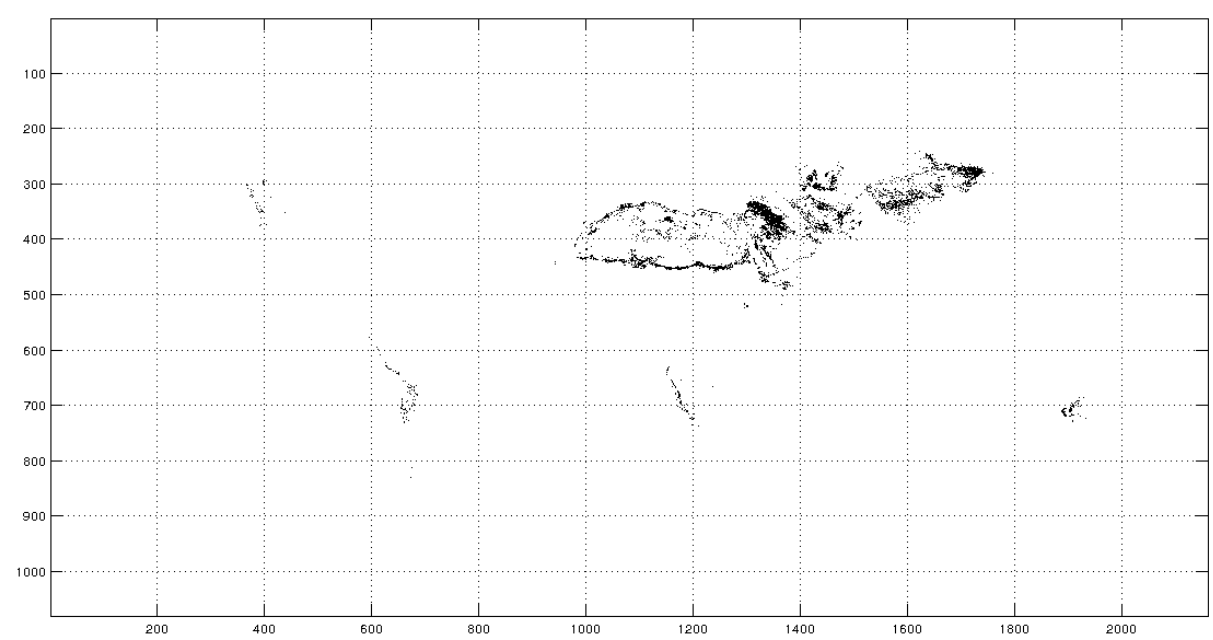

(a) FAPAR binary map estimated using the determinism rates.

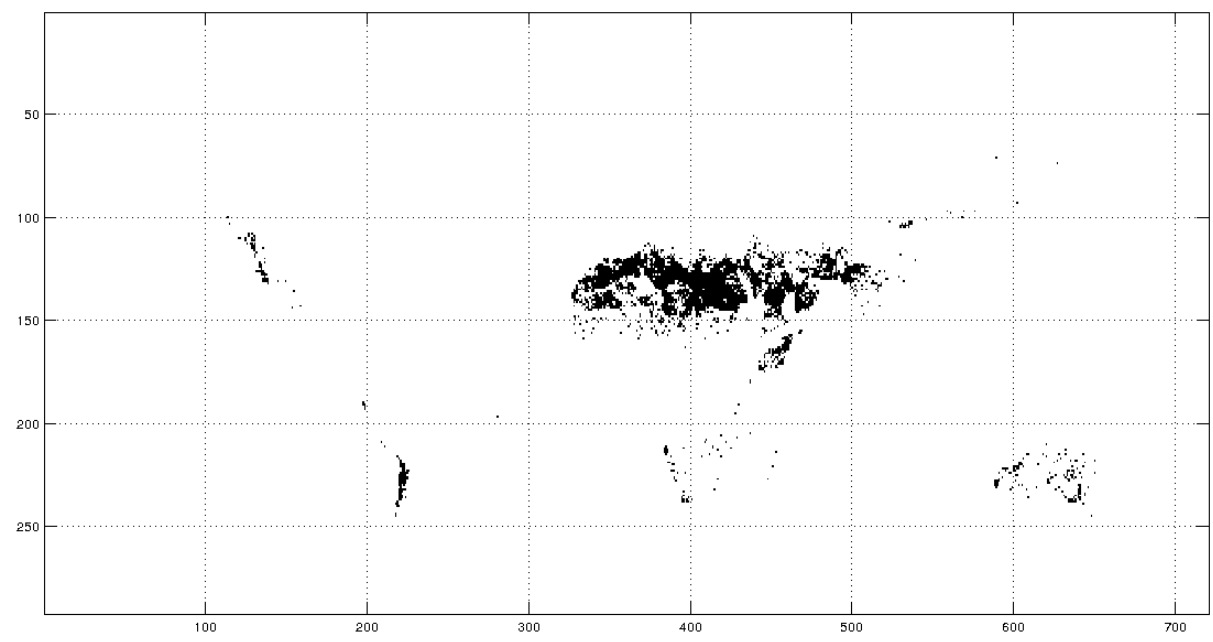

(b) Rainfall binary map estimated using the determinism rates.

Figure 6.6: Binary 2D World maps obtained after applying the determinism rate on FAPAR and Rainfall datasets.

sea are represented by letters $c$ (continental) and $m$ (maritime), respectively. The second letter, used in uppercase, represents the temperature. Tropical and polar masses are defined by the letters $T$ and $P$, respectively. Hence, by using this notation, it is possible to represent the following types of air masses: $c T, m T, c P$, and $m P$. There still exist two other types of air masses: $m E$ and $c A$, in which letters $A$ and $E$ stand for the Arctic/Antarctic and Equatorial influences, respectively. The influences of air masses on Earth is shown in Figure 6.8.

By analyzing determinism rates considering the global temperature dataset in conjunc- 


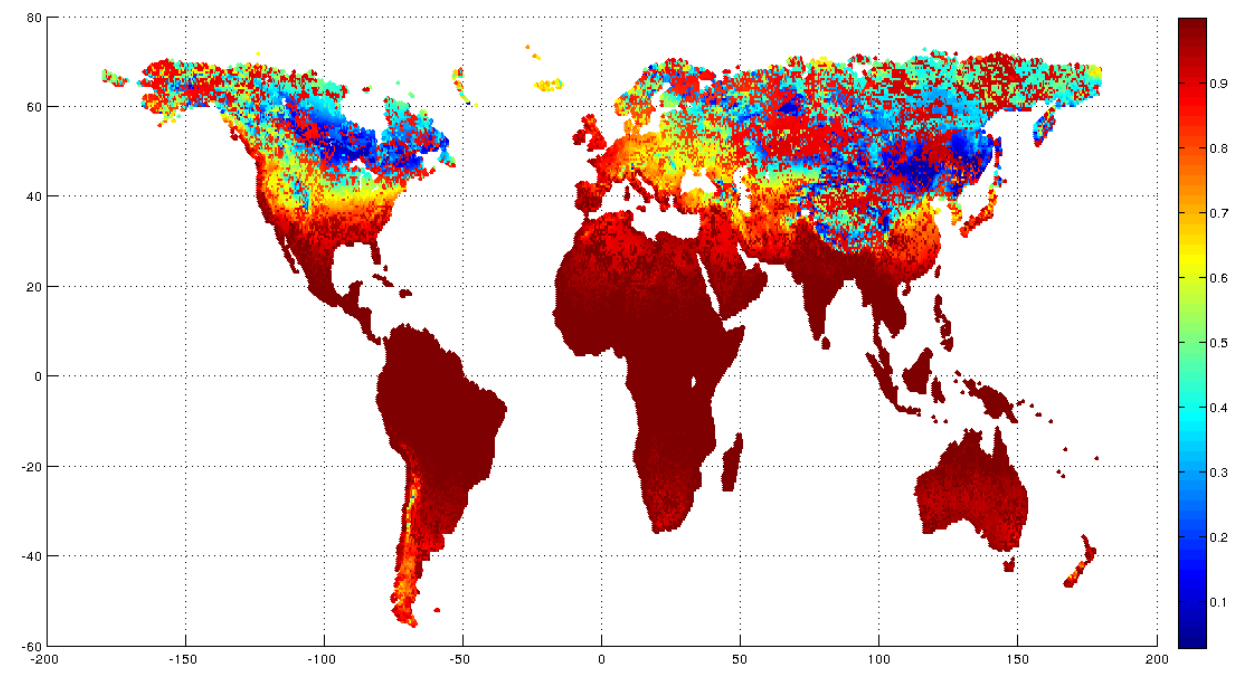

Figure 6.7: Deterministic 2D World map obtained with EMD-MI on Temperature dataset.

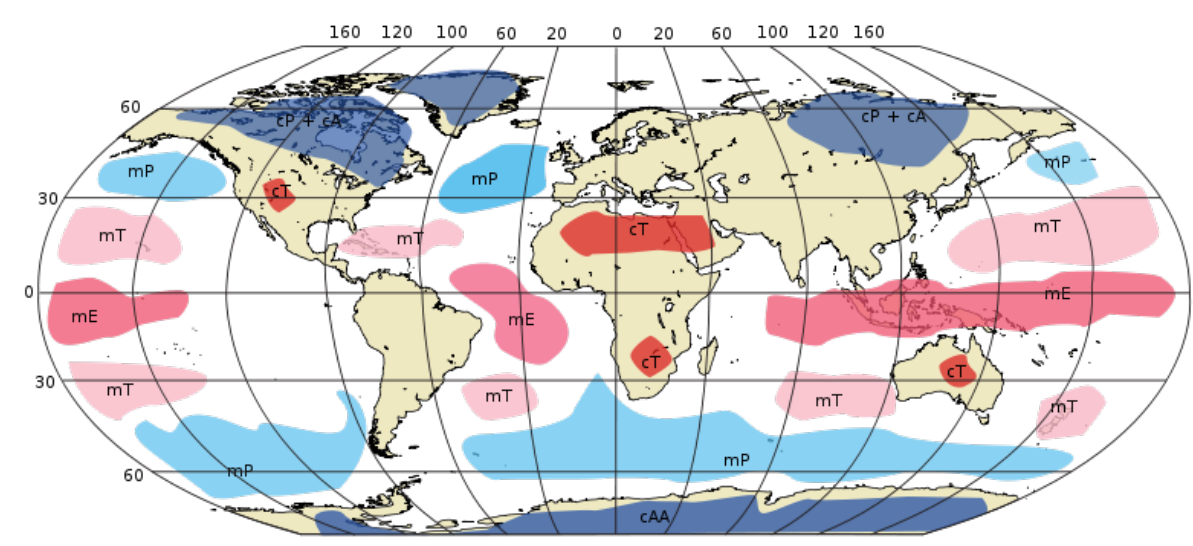

Figure 6.8: Global air masses. Figure provided by NASA under public domain license.

tion with air mass influences, we conclude there is a correlation between the stochasticity and the presence of polar masses. On the other hand, when a region is influenced by tropical or equatorial masses, the determinism rate is higher, indicating deterministic behavior.

Besides this correlation, by using the determinism rate to continuously analyze a flow of temperature data, it is possible to detect weather fronts, which are imaginary limits between two masses of different characteristics (Ahrens, 2009). Once such limits are known, several meteorological phenomena can be better understood. 


\subsubsection{Discussion}

The results presented in this chapter confirmed the EMD-MI approach is useful to identify regions characterized by a transition between different environments. For example, if we look at the deterministic 2D World map for the FAPAR dataset, we observe the highest stochastic levels correspond to transition regions in between deserts and areas with higher concentration of vegetation. The same conclusion is drawn by looking at the deterministic 2D World map obtained for the Rainfall dataset, i.e., in similar regions on both maps, the incidence of rain is as much stochastic as the fraction of absorbed radiation (see Figure 6.6).

Among the regions whose the deterministic rate is low, we have the Sahel region, which has been studied by several researchers (Heumann et al., 2007; Anyamba and Tucker, 2005; Gonzalez et al., 2012). Sahel is a semi-arid region that represents a transition zone between the Sahara desert and the humid tropical savanna and it is located at the north of the African continent. However, the precise geographic location of the Sahel is difficult to distinguish because its physical characteristics change over time and space as discussed in (Anyamba and Tucker, 2005). Therefore, by automatically selecting this region, one can monitor the progress of arid region over time. According to Gonzalez et al. (Gonzalez et al., 2012), the increased aridity and human population have reduced tree cover in parts of the African Sahel and degraded resources for local people.

Sahel is also characterized by low and variable rainfall. As discussed in (Heumann et al., 2007), the mean annual rainfall in Sahel decreased markedly between early 1960s and mid 1990s, compared to the first half of the 20th century. This downturn in rainfall has called the attention of researchers to examine the influence of land surface vegetation on the general precipitation behavior (Heumann et al., 2007), confirming the correlation found out by our approach.

Although this correlation between the FAPAR and Rainfall datasets is known by researchers, it is difficult to measure it by using conventional techniques, once the dynamics defining the time series behavior are different for each system. This difference is noticed by analyzing the time series presented in Figure 6.9, which contains two plots. The first one (Figure 6.9(a)) shows a time series extracted from FAPAR dataset, whereas the second (Fig- 
ure 6.9(b)) corresponds to a time series from the Rainfall dataset. This difference between the dynamics of datasets did not influence our approach, which correlated these time series, once the estimated determinism rates were $R_{\mathrm{det}}=0.503$ and $R_{\mathrm{det}}=0.512$, respectively.
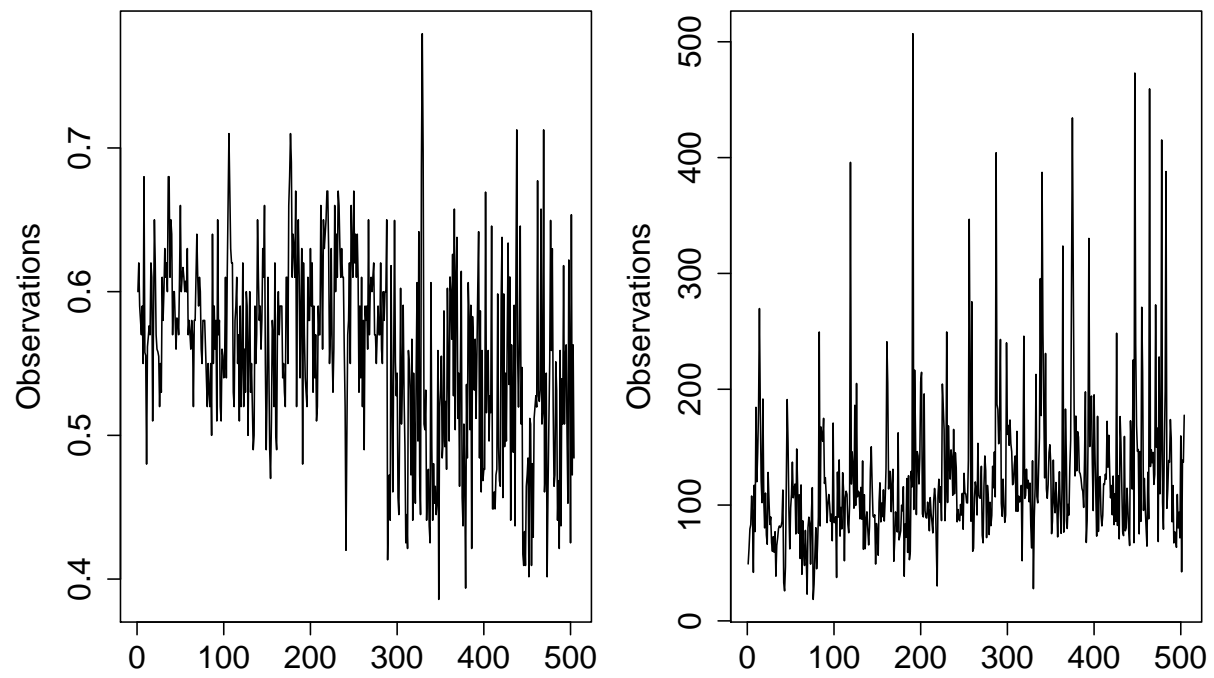

(a) FAPAR

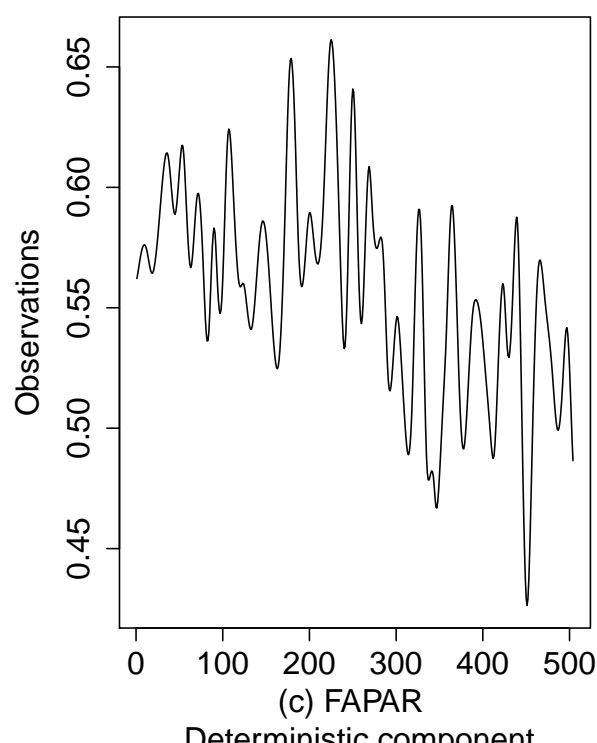

(b) Rainfall

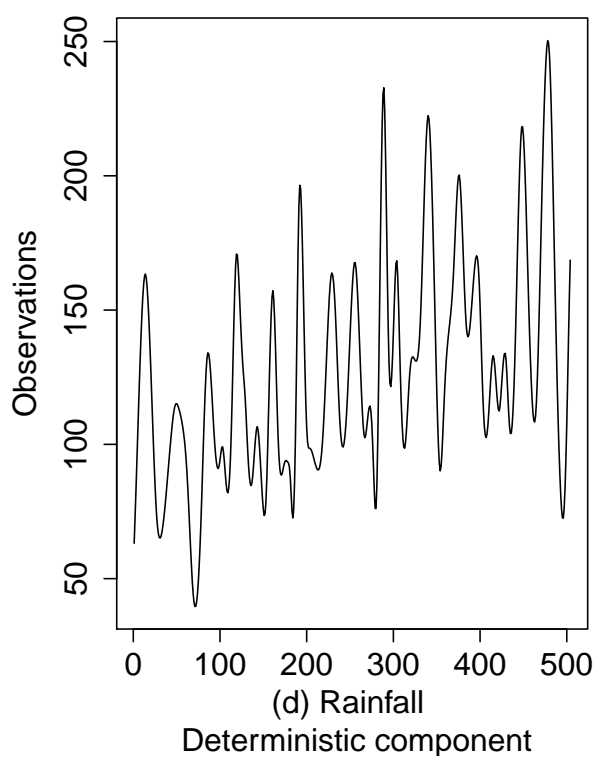

Figure 6.9: A sample of original time series selected from FAPAR (a) and Rainfall (b) datasets. We observe their dynamics are completely different, although they have similar stochastic characteristics.

Finally, besides the advantages previously presented, the proposed approach is also useful to model general-purpose time series. In this scenario, one can separate the stochas- 
tic and deterministic components and adjust specific models to each one of these behaviors (Han and Liu, 2009). This approach tends to increase the modeling accuracy, once the influence of individual components are separately estimated. In this work, however, we did not perform this analysis, because the modeling process is out of the scope of this thesis.

\subsection{Concluding remarks}

In this chapter, we presented the application of the EMD-MI approach and the $R_{\text {det }}$ measure on real-world application, whose results confirmed their importance in decomposing time series and evaluating the stochastic and deterministic influences, respectively. This again confirms the first part of our hypothesis. On the other hand, the second part was addressed in Chapter 5.

By using the EMD-MI approach and the determinism rate, we were able to detect important patterns in FAPAR and Rainfall datasets involving semi-arid regions and tropical rain forests. Furthermore, experiments also detected some regions of interest such as Sahel, located at North Africa. The automatic detection of this region is not a simple task as discussed in Section 6.3.4. We also correlated semi-arid regions and places in which the stochasticity is high in terms of rainfall. This allowed to identify similar patterns in different datasets, in which the application of traditional techniques tends to present problems due to the different dynamics that characterize datasets. Finally, after applying our decomposition approach on the Temperature dataset and estimate the determinism rate, we noticed regions with deterministic behavior are influenced by equatorial and tropical air masses. By understanding the determinism rates for the temperature data and the influences of air masses, one can study weather fronts, which play an important role in the analysis of several weather phenomena. 


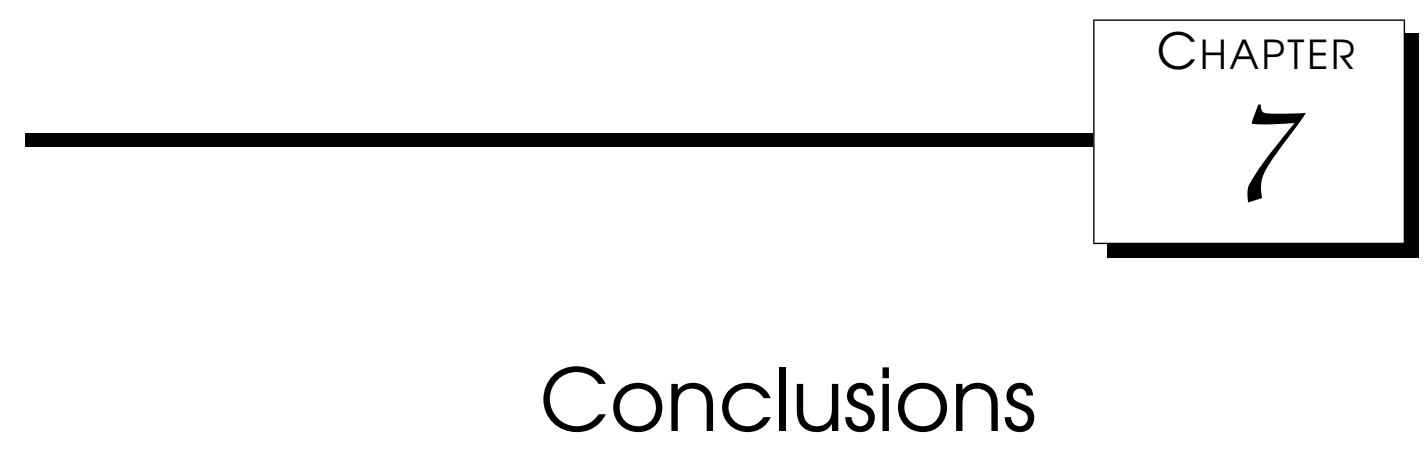

\subsection{Concluding remarks}

This thesis demonstrated the analysis of stochastic and deterministic influences of time series is an important step to model and understand system behavior. In this sense, several approaches have been proposed to model time series considering either the stochastic or the deterministic influence present in observations. However, those models tend to be ill-adjusted in scenarios characterized by the presence of both influences simultaneously, once relevant information is disregarded.

In order to consider the stochastic and the deterministic influences on time series modeling, we conducted this research based on three well-defined steps suggested by Wangler and Backlund (2005): i) decompose time series into stochastic and deterministic components; ii) individually adjust models on every component; and iii) combine the adjusted models to form a hybrid one in attempt to better describe global time series behavior. Considering these three steps, we designed the hypothesis, which states the individual modeling of stochastic and deterministic components permits obtaining a hybrid one with 
higher accuracy.

This hypothesis was proved in two steps. The first one was related to the process of decomposing a time series into two additive components: one stochastic and another deterministic. The second one is related to the evaluation of the hybrid model obtained after individually analyzing both components.

Aiming at proving the first part of our hypothesis, we performed a search for decomposition methods according to the Systematic Literature Review (SLR) (Rios and Mello, 2012). Based on this review, we noticed Empirical Mode Decomposition (EMD) (Huang et al., 1998) is a very useful method to decompose time series regardless the system behavior used to produce observations. By analyzing the results obtained by applying EMD on time series, we noticed the stochastic influence tends to reduce as components (Intrinsic Mode Functions - IMFs) are extracted. Considering this empirical observation, we developed a formal study based on the Nyquist-Shannon sampling theorem (Jerri, 1977) to prove components produced by EMD can be separated according to high and low frequencies, representing stochastic and deterministic influences, respectively. The results obtained from this proof supported the development of two new approaches to decompose time series into stochastic and deterministic components.

Experiments evaluated both approaches and confirmed the decomposition process positively impacts time series modeling, once the stochastic and deterministic influences present in time series are better understood. Models estimated on individual components were combined to form a hybrid model, which outperforms the modeling of undecomposed time series in most of the studied scenarios. In other situations, either the deterministic or the stochastic model outperforms the undecomposed time series, because one of them dominates the other. These results allowed us to prove the second part of our hypothesis.

Furthermore, another important consideration about our decomposition approaches is the possibility of using them to filter time series and remove noise. Although this usage is not considered in this thesis, it is common when one wishes to analyze only the deterministic component, ignoring the stochastic one which is taken as noise in certain application domains.

After proving our hypothesis, we also performed experiments on real-world datasets, which confirmed the importance of decomposing time series and evaluating the stochastic 
and deterministic influences, as stated by the first part of our hypothesis. In these experiments, we verified both components provide important information to detect patterns and extract implicit information of time series.

In order to evaluate both approaches, we designed two new measurements. The first one was motivated by the limitation of existing measurements in detecting the efficiency of models when forecasting observations. In general, most of the measurements consider the perfect match between expected and predicted values. However, there are situations in which, even providing different results, the predicted values follow the same trend and seasonality as the time series. This first measurement handles these issues. The second measurement is important to assess the influence of the deterministic and stochastic components on time series observations.

Finally, the research developed in this thesis also motivated us to investigate the use of decomposition methods to produce surrogate data (Appendix C). Although this study on surrogates is not directly related to this work, it was also important to improve our knowledge on time series analysis.

\subsection{Publications and collaborations}

This section presents the manuscripts and collaborations performed during the development of this research. The following list contains all papers published and under review:

- Ishii, R. P.; Rios, R. A.; Mello, R. F. Classification of time series generation processes using experimental tools: a survey and proposal of an automatic and systematic approach. International Journal of Computational Science and Engineering, v. 6, p. 217-237, 2011.

- Rios, R. A.; Mello, R. F. Analysis of Decomposition Techniques to Estimate Time Series Stochastic and Deterministic Components: A Systematic Review. In: Escola de Séries Temporais e Econometria, 2011, Gramado. 14a Escola de Séries Temporais e Econometria, 2011.

- Rios, R. A.; Mello, R. F. Time series decomposition in terms of stochastic and deterministic components: an approach using recurrence plot and empirical mode de- 
composition. In: Fourth Recurrence Plot Symposium, 2011, Hong Kong. Fourth Recurrence Plot Symposium, 2011.

- Rios, R. A.; Mello, R. F.. An Empirical Approach using Spectral and Recurrence Analysis to Estimate Time Series Stochastic and Deterministic Components. In: Conferência Brasileira de Dinâmica e Controle, 2011, Águas de Lindóia. X Conferência Brasileira de Dinâmica, Controle e Aplicaćões, 2011.

- Rios, R. A.; Mello, R. F. A Systematic Literature Review on Decomposition Approaches to Estimate Time Series Components. INFOCOMP, v. 11, p. 31-46, 2012.

- Rios, R. A.; Mello, R. F. Improving time series modeling by decomposing and analyzing stochastic and deterministic influences. Signal Processing (Print), v. 93, p. 30013013, 2013.

- Rios, R. A.; Small, M.; Mello, R. F. Testing for linear and nonlinear Gaussian process in nonstationary time series (under review).

- Rios, R. A.; Parrott, L.; Lange, H; Mello, R. F. Estimating determinism rates to detect patterns in geospatial datasets (under review).

- Rios, R. A.; Mello, R. F. A Formal Analysis on Stochastic and Deterministic Components Decomposed from Signals (under review).

The collaborative work started with Prof. Michael Small during a visit to the Department of Electronic and Information Engineering at the Hong Kong Polytechnic University in 2011. As consequence of this visit, we designed two new methods to generate surrogate data, which are presented in Appendix C. This visit was supported through the research contingency funds related to the doctorate fellowship provided by FAPESP under grant 2009/18293-9. Recently, Prof. Small is with the School of Mathematics and Statistics at the University of Western Australia.

The second collaborative work was conducted with Prof. Lael Parrott and Prof. Holger Lange during a visit to the Université de Montréal - Canada. This visit was supported by CAPES (Coordination for Enhancement of Higher Education Personnel), which provided a scholarship for a doctoral internship as part of the Program Doctoral Sandwich Abroad (PDSE) under grant 5983-11-8. Currently, Prof. Parrott leads the Complex Environmental 
Systems Laboratory with the Department of Earth \& Environmental Sciences and Biology at the University of British Columbia (Canada) and Prof. Lange is with the Norwegian Forest and Landscape Institute (Norway). This visit allowed us to design a decomposition approach and the Determinism Rate measurement presented in Section 4.3.1 and 4.3.2, respectively. In addition, this visit also allowed us to evaluate our approach and measurement on real-world datasets as presented in Chapter 6.

\subsection{Future work}

Several opportunities arise from this work, among them it is worth mentioning the importance of studying attractors formed by the deterministic components. During experiments using EMD, we noticed the attractor in phase space may change depending on the stochastic influence present in time series. This change happens due to the decomposition method used to extract components. The EMD method, adopted in this thesis, extracts IMFs that behave similarly to waves or sinusoidal series. Consequently, in situations in which the stochastic component brings a stronger influence on observations, the deterministic component tends to behave similarly to a sine function. Therefore, this highlights a new gap which motivates the development of approaches to reduce the impact of this bias imposed by the EMD method.

Furthermore, this problem is not only faced by EMD, what requires another study to map the bias imposed by different decomposition methods. By knowing such bias in advance, one can choose the method whose behavior of the decomposed component is closer to the expected one. Finally, this also permits designing new measurements to evaluate similarities between attractors obtained after and before decomposition. By using such measurements, one can, for instance, evaluate the model accuracy considering the stochastic influence on time series observations taking into account their behavior in phase space and not only the individual value as usually adopted by traditional measurements like the signal-to-noise ratio (SNR) (Gonzalez and Woods, 2006). 


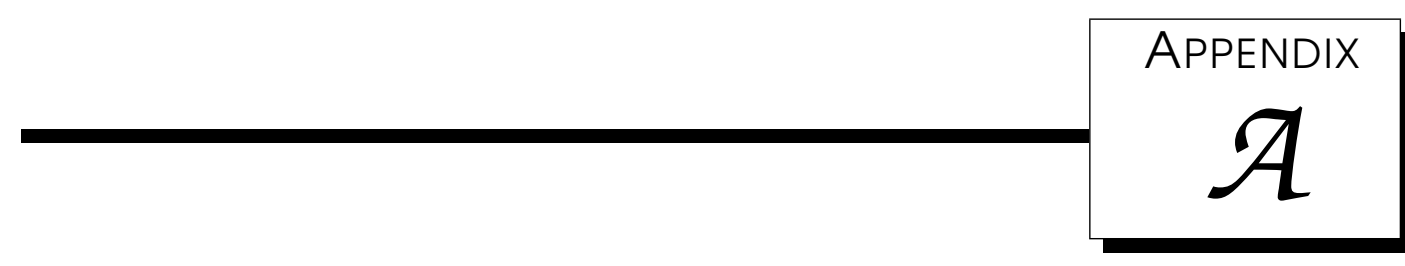

\section{Systematic Literature Review}

\section{A. 1 Introduction}

Many researchers have been proposing new techniques to decompose time series, aiming at separating stochastic and deterministic components. This increasing number of publications motivated this work, whose main objective is to identify the most useful techniques to perform such decomposition. In that sense, we conducted a structured and documented study of published papers following the rules defined by the method of Systematic Literature Review (SLR) (Kitchenham et al., 2009).

The SLR method is commonly employed in many areas, such as medicine. Its goal is to define a rigorous and well-structured search for published papers in order to collect and evaluate evidences on a given subject. The main advantage of SLR is to perform a wide search, recovering not only well-known papers but also other related ones. Papers collected by SLR can be either directly read and analyzed or organized as a Systematic Mapping, as presented in (Petersen et al., 2008; Durelli et al., 2010).

By employing SLR, this appendix presents strong evidences of the importance of decomposing time series and also identifies the most common methods considered for this 
purpose, the frequency of published papers, the relation in between the number of papers and the type of publication, and the most important gaps of each method (Rios and Mello, 2012). All discussion presented in this chapter was based on the results published in (Rios and Mello, 2012).

\section{A.2 Systematic Review}

In medicine, the process of clinical decision is commonly performed according to the methodology called Evidence-Based Medicine (EBM) (Evidence-based Medicine Working Group, 1992). This methodology was developed after researchers concluded that the specialist's opinion based on a medical advice is as trustful as the opinion obtained from scientific experiments (Kitchenham et al., 2009). Considering this assumption, EBM was created to reduce the importance of non-systematic clinical experience on decision making. EBM also increased the importance of the evidence analysis obtained from clinical research. The application of this methodology requires new abilities of the physician as, for instance, the efficient research in scientific literature and the application of formal rules when evaluating evidences (Dyba et al., 2005).

The advantages obtained with EBM have motivated the adoption of this methodology in other areas such as Criminology, Economy and Nursing (Kitchenham et al., 2009). In Computer Science, specifically in Software Engineering, Dyba et al. (2005) defined, based on EBM, a methodology called Evidence-Based Software Engineering (EBSE). According to the authors, the main objective is to provide ways by which the best evidences gathered from different researches can be integrated to human values and practice experiences, aiming at improving software development and maintenance.

According to Kitchenham et al. (2009), an evidence can be defined as a synthesis of highquality scientific studies about a given research topic. The main synthesis method defined by this methodology is the Systematic Literature Review (SLR), which provides guidelines to make a rigorous review of studies related to the topic of interest. It is important to highlight that SLR cannot substitute the traditional review of literature, since this review is necessary before executing a rigorous search and defining which papers are relevant for the review. In addition to that, traditional review provides some initial concepts about 
the studied subject, which makes it possible to choose keywords and define inclusion and exclusion criteria used in SLR, and, eventually, to add some specific and important studies, which were not retrieved by SLR.

However, SLR is limited in the sense that is restricted to some previously defined keywords, which can eventually lead to the lack of some important related work. An approach commonly considered to overcome such problem is to execute a recursive search; thus, references of a selected paper are then used to retrieve further studies.

In this sense, aiming at developing a systematic and rigorous research on time series decomposition, the guidelines provided by SLR, presented in (Kitchenham et al., 2009; Endo and da Silva Simão, 2010), were adapted and divided in three phases: in the first, called Search, some criteria were defined to seek related work; in the second, called Analysis, the quality of gathered papers was analyzed and quantified; finally, the last phase presents conclusions obtained from the systematic study. In the following sections, each phase is presented and discussed in details (Rios and Mello, 2012).

\section{A.3 Phase I: Search}

In the first phase, we defined the general scope of the research, i.e., we selected the research criteria to characterize whether a paper is related to this study or not. In this sense, we defined the objective of this research, the main and secondary research questions, the search repositories, the standard language, the list of keywords, the search query, the inclusion and exclusion criteria, and, finally, the general process of execution.

In general, the main objective of this research is to find techniques that are related to the decomposition of time series in terms of stochastic and deterministic components. Based on this objective, the Main Research Guestion (MRQ) to guide this research is (Rios and Mello, 2012):

What are the techniques used to decompose time series in stochastic and deterministic components?

In addition to this main question, it is relevant to define a set of Secondary Questions (SQ), which are directly associated to the validity of the proposed research. This set of questions aims at discovering practical applications of the research, evaluating techniques, and 
understanding publication trends. The secondary questions are (Rios and Mello, 2012):

SQ.1 - What types of practical applications can take advantage of this decomposition process?

SQ.2 - How are techniques evaluated?

SQ.3 - Why are time series decomposed?

SQ.4 - What are the main techniques used to decompose time series?

SQ.5 - What is the frequency of published papers per year?

SQ.6 - Who are the main researchers in this area?

SQ.7 - What are the limitations of techniques?

Due to the complexity and importance of the main objective of this research, the last secondary question was divided, as in the study presented in (Kitchenham et al., 2009), into the following four questions (Rios and Mello, 2012):

SQ.7.1 - Was the search query limited?

SQ.7.2 - Is there any evidence that the studied subject was limited by the lack of primary studies $^{1}$ ?

SQ.7.3 - Is the quality of the studied subject good enough?

SQ.7.4 - What are the main limitations of the found approaches/techniques?

After defining these questions, the next step of this first phase was to choose the search repositories, from which related studies were obtained. We selected repositories which provide Web search engines, accept queries using keywords, and are commonly used by the scientific community. Based on these restrictions, the following repositories were chosen (Rios and Mello, 2012):

- Scopus (http://www.scopus.com/home.url);

\footnotetext{
${ }^{1}$ According to the guidelines of the SLR presented in (Kitchenham et al., 2009), a primary study is a work that contributes to a new approach or technique of a given subject. On the other hand, reviews of existing approaches, such as SLRs and Surveys, are considered secondary studies.
} 
- ACM Digital Library (http://portal.acm.org/);

- IEEE Xplore Digital Library (http://ieeexplore.ieee.org/).

The standard language used in this systematic review was English, i.e., all papers written in other languages were discarded. As the next step, the following keywords were chosen considering the hypothesis and the main research question (Rios and Mello, 2012):

- Data organization: time series;

- Goals: decomposition, filter;

- Results: deterministic, and stochastic.

We have also decided to introduce the term "filter" as synonym to decomposition because, according to our previous studies, this term is frequently used to refer to techniques that decompose time series into stochastic and deterministic components. Of course, there are several synonyms that could be added as keywords, but they would considerably increase the number of returned papers, making it almost impossible to perform this study. This is also foreseen by SLR, which limits the number of keywords and make the systematic research feasible (Rios and Mello, 2012).

Based on keywords, the following search query was defined (Rios and Mello, 2012):

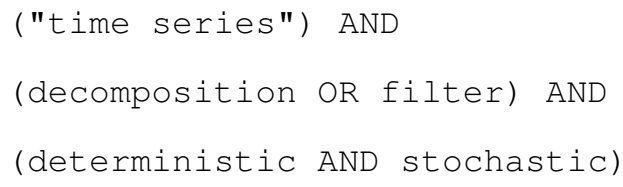

We then use this query to obtain relevant papers from the repositories. In spite of the reduced number of keywords, our query has returned a high number of papers, however many of them were not relevant to SLR. Aiming at reducing this number, we defined inclusion and exclusion criteria. Therefore, a work was added to SLR when it satisfied the following conditions (Rios and Mello, 2012):

- Does the work separate stochastic and deterministic components from a time series?

- Is it a primary study?

- Is the work focused on the modeling process of time series? 
On the other side, a paper must be discarded if any of the following exclusion criteria is applied (Rios and Mello, 2012):

- The work presents a technique which is very restricted to a specific problem;

- The work does not present a well-defined analytical model;

- The technique evaluation process is not satisfactory;

- The work does not have a comprehensive literature review.

Finally, after defining all initial conditions, the first phase of SLR, the search phase, was carried out according to the following steps (Rios and Mello, 2012):

1. The defined query was applied in the selected repositories;

2. The title and abstract of each paper were analyzed, considering the inclusion and exclusion criteria;

3. The redundant papers were removed from SLR;

4. The remaining papers were completely read.

It is important to highlight that a given paper, selected to compose SLR, can still be removed in step 4 if an exclusion criteria is applied to it, even after being completely read. In the same way, if references of selected papers are considered relevant to SLR, they are indeed included in the list of selected papers, aggregating studies to SLR that were not found when searching in repositories. The next section presents the analysis of papers selected in this first phase.

\section{A.4 Phase II: Analysis}

The application of the search query, defined in the first phase, on the selected repositories returned 852 papers, which were distributed in repositories as presented in Table A.1. However, some of the obtained references were not, in fact, valid, because they were proceedings, information on editorial boards or redundant papers. Therefore, after removing these invalid references, the total of remaining papers was 749 . We then analyzed every paper, by the reading of their titles and abstracts, and the use of the inclusion criteria. After 
this analysis, 77 papers were selected to be completely read and evaluated. At last, part of these papers were still rejected after applying the exclusion criteria. Hence, we finally classified 26 papers as strongly related to the subject presented in this work.

Table A.1: Number of papers returned by SLR. "Recovered papers" represents the number of papers returned by SLR, after removing invalid references. "Inclusion criteria" represents the number of papers selected after reading titles and abstracts. Finally, "Exclusion criteria" shows the number of remaining papers after being completely read.

\begin{tabular}{lr}
\hline Repository & Number of papers \\
\hline ACM & 773 \\
IEEE & 66 \\
Scopus & 13 \\
Total & 852 \\
\hline Recovered papers & 749 \\
After inclusion criteria & 77 \\
After exclusion criteria & 26 \\
\hline
\end{tabular}

First of all, we analyzed the 26 selected papers to understand the frequency distribution of authors per country, which is presented in Table A.2. All the analysis performed on the 26 selected papers were also executed on the other papers: all the 749 papers and those ones selected by the inclusion criteria (77), which are available in (Rios and Mello, 2012).

Table A.2: Frequency distribution of authors per country.

\begin{tabular}{rr}
\hline & Frequency distribution \\
\hline US - United States & 20 \\
CN - China & 6 \\
DE - Germany & 6 \\
FR - France & 6 \\
UK - United Kingdom & 5 \\
ES - Spain & 4 \\
IT - Italy & 4 \\
BR - Brazil & 3 \\
GR - Greece & 3 \\
CA - Canada & 2 \\
CH - Switzerland & 2 \\
IN - India & 2 \\
PT - Portugal & 2 \\
SE - Sweden & 2 \\
AT - Austria & 1 \\
AE - United Arab Emirates & 1 \\
KR - Korea (South) & 1 \\
NL - Netherlands & 1 \\
TW - Taiwan & 1 \\
\hline
\end{tabular}


Table A.3 presents another interesting result, ordered by researchers with more papers related to the time series decomposition in terms of stochastic and deterministic components. This analysis was useful to answer the secondary question SQ.6, which aims at presenting the main researchers in this area (Rios and Mello, 2012).

Table A.3: List of authors who published more papers.

\begin{tabular}{rr}
\hline & Frequency distribution \\
\hline Norden E. Huang & 4 \\
George Tzagkarakis & 2 \\
Maria Papadopouli & 2 \\
Panagiotis Tsakalides & 2 \\
Steven R. Long & 2 \\
Zhaohua Wu & 2 \\
\hline
\end{tabular}

Table A.4 presents the frequency of papers published per year, what is important to answer the secondary question SQ.5. According to this table, 2006, followed by 2009 , were the years with more publications (Rios and Mello, 2012).

Table A.4: Number of papers published per year.

\begin{tabular}{cr}
\hline & Frequency distribution \\
\hline 1995 & 1 \\
1998 & 1 \\
2000 & 3 \\
2001 & 2 \\
2003 & 1 \\
2004 & 1 \\
2005 & 1 \\
2006 & 6 \\
2007 & 1 \\
2009 & 5 \\
2010 & 2 \\
2011 & 2 \\
\hline
\end{tabular}

Table A.5 shows the frequency distribution of selected papers considering the type of publication. In this case, we observe that most of the papers were published in journals (Rios and Mello, 2012).

The next analysis (Table A.6), executed after the identification of the type of publication, was performed to know how many papers were primary studies (Rios and Mello, 2012).

Aiming at answering the secondary question SQ.3, in Table A.7, we analyzed the relation between published papers and goals when applying decomposition techniques (Rios and 
Table A.5: Relation between the number of papers and the publication type.

\begin{tabular}{rr}
\hline & Frequency distribution \\
\hline Journal & 21 \\
Conference & 3 \\
Book Chapter & 1 \\
Symposium & 1 \\
\hline
\end{tabular}

Table A.6: Type of published papers.

\begin{tabular}{rr}
\hline & Frequency distribution \\
\hline Primary Study & 22 \\
Application & 4 \\
\hline
\end{tabular}

Mello, 2012).

Table A.7: Goals of publications.

\begin{tabular}{rr}
\hline & Frequency distribution \\
\hline Denoising & 8 \\
Filtering & 2 \\
Decomposition & 13 \\
Modeling & 3 \\
\hline
\end{tabular}

All these analysis compose an overview on papers obtained using SLR. However, to proceed with our study, we had to read and understand the details of each selected paper. This further analysis was conducted based on some questions, which were elaborated aiming at answering secondary questions SQ.1, SQ.2, and SQ.4. Hence, we defined the following questions (Rios and Mello, 2012):

- What is the context of the paper?

- What are the techniques used by the new method?

- How is the new method described?

- What are the goals?

- How is the new technique evaluated?

- What is the score attributed to the paper?

The score of every paper was limited in the interval $[0,6]$, in which 0 means very poor and 6 very good. This score was defined by the sum of other specific questions, whose 
objective was to evaluate the quality of the paper in relation to references, the reproducibility of results, the quality of obtained results, the description, formalism and analysis of the proposed method. So, aiming at quantifying the quality of each paper, every specific question was classified as fair (0), average (0.5), or good (1).

The general results of this analysis are summarized in (Rios and Mello, 2012). From these results, we observed decomposition techniques are very useful to improve a variety of applications, answering in this way secondary question SQ.1. For example, according to de Lima et al. (2006), the decomposition of time series in stochastic and deterministic components has been used to study tremor in the human being bodies. In this situation, every component represents a type of tremor (voluntary or not), thus, decomposition supports such identification. In another scenario (Meyer and Stiedl, 2006), decomposition is used to understand the dynamics of heartbeat interval fluctuations in awake unrestrained mice, following the intracerebroventricular application of the neuropeptide Corticotropin-Releasing Factor (CRF). Other two examples in Biology, discussed by Kopsinis and McLaughlin (2009) and Gruber et al. (2006), used stochastic and deterministic components to, respectively, understand the behavior of bats and analyze protein chains. Another very interesting study using the decomposition of time series is presented in (Amman and Das, 2001), which aims at modeling automotive engine sounds. In Computer Science, time series decomposition has been used in several studies as, for instance, to understand behavior patterns of messages in Wifi networks (Tzagkarakis et al., 2007; Chung et al., 2010).

Besides providing some real and practical examples of time series decomposition, the selected papers were still useful to show us the most common techniques used to evaluate experimental results. In this sense, after decomposing a time series, each component was evaluated using not only a visual inspection but also statistical techniques, Mean Squared Error (MSE) (de Lima et al., 2006; Nounou, 2006; Amman and Das, 2001; Macciotta et al., 2000), Signal-to-Noise Ratio (SNR) (Nounou, 2006; Kopsinis and McLaughlin, 2009; Moon and Weissman, 2009) and Recurrence Guantification Analysis (RQA) (Liu and Liao, 2011). MSE is used, in general, to evaluate the results obtained when predicting new observations. In this situation, a time series is decomposed and, then, each component is individually modeled and used to predict future values. Thus, MSE is employed to compare expected 
values against predicted ones. In contrast, SNR is normally considered to assess the precision of removing the stochastic component from a time series. In such situation, a time series is decomposed into stochastic and deterministic components. The expected values of the deterministic component, which is known a priori, are compared against the estimated ones. At last, RQA is a set of measures, defined on the results of the Recurrence Plot (RP) technique (Marwan et al., 2007), which aims at quantifying the relation between stochastic and deterministic signals. One of the most used measure is the determinism rate (DET), that quantifies the influences of noise (or stochastic component) on time series, i.e. stochastic components present lower DET values than deterministic ones.

Finally, secondary question SQ.4 aims at determining what techniques are most used to decompose time series. As presented in (Rios and Mello, 2012), the most used techniques are: Fourier (Macciotta et al., 2000) and Wavelet (Aminghafari et al., 2006; Han and Liu, 2009; Kopsinis and McLaughlin, 2009) Transforms, Principal Component Analysis (PCA) (Liu and Liao, 2011; Tzagkarakis et al., 2007) and Empirical Mode Decomposition (EMD) (de Lima et al., 2006; Kopsinis and McLaughlin, 2009).

\section{A.5 Phase III: Conclusion}

The conclusion obtained from the application of the SLR method and the complete results are detailed in (Rios and Mello, 2012). In summary, the SLR method permitted concluding spectral analysis methods are the most commonly considered to decompose time series (Rios and Mello, 2012). A study on these spectral methods was performed in Chapter 2. 


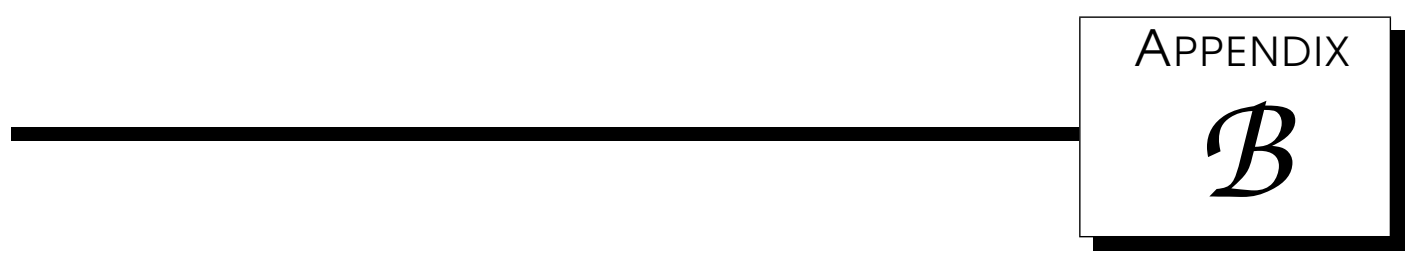

\section{Dynamical System Background}

This appendix briefly introduces concepts on dynamical systems in order to support the study of time series into phase spaces (also referred to as time-delay coordinate spaces) (Alligood et al., 1997; Mello, 2009). Those spaces were initially studied by Whitney (1936), who applied differential manifolds to reconstruct functions into multidimensional spaces. Based on this reconstruction Whitney (1936) proposed his immersion theorem, which states attractors are better understood when time series are unfolded into a highdimensional space.

According to Alligood et al. (1997), attractors are defined by the presence of fixed points and orbits that define how time series observations evolve over time. In this sense, a fixed point is defined as:

Definition: Let $f$ be a map in $\mathbb{R}$ and $p$ be a number such that $f(p)=p$. If all points close to $p$, taking into account a neighborhood $\mathcal{V}$, are attracted to $p$, then $p$ is referred to as an attracting fixed point. On the other hand, if all points move away from $p$, then it is called repelling fixed point. 
Similarly, an orbit is a region in the phase space, in which observations are attracted or repelled.

Based on Whitney's studies, Takens (1980) proved his immersion theorem in which a time series $\left\{x_{0}, x_{1}, \ldots, x_{n-1}\right\}$ can be reconstructed into a phase space $x_{n}(m, \tau)=$ $\left\{x_{n}, x_{n+\tau}, \ldots, x_{n+(m-1) \tau}\right\}$, having $m$ as the embedded dimension and $\tau$ representing the time delay (or delay dimension or separation dimension).

The embedded dimension basically defines the number of axes necessary to unfold time series into the phase space. The delay dimension, on the other hand, is important to represent the seasonal behavior of time series, indicating the necessary displacement among past observations.

The estimation of embedded dimension was studied by Takens (1980) and Mañé (1980), who confirmed the upper limit for the embedded dimension $D_{e} \in \mathbb{N}$ can be defined by fractal dimension $D_{f}$ according to equation $D_{e}>2,0 \cdot D_{f}$. However, studies conducted by Kennel et al. (1992) demonstrated the dimension obtained from this equation is, in general, greater than necessary, adding more complexity and execution time when analyzing the corresponding phase space (Kennel et al., 1992).

In order to overcome this drawback, Kennel et al. (1992) proposed the False Nearest Neighbors (FNN) method, which analyzes the neighborhood for every observation in the phase space. In summary, this method starts calculating the distance among observations considering the embedded dimension equals to one. Then, a new dimension is added and distances are again calculated. If distances increase, observations are considered as false neighbors, i.e., observations that lay close together are separated in higher embedding dimensions, eliminating the false neighbors and making evident the need for a higher dimensional reconstruction. If by adding a new dimension, the false neighbor rate is zero, then the total of dimensions is taken as embedded dimension.

Formally, the False Nearest Neighbors method considers an embedded dimension $m$, in which the $r$-th neighbor close to $y(n)$ is defined by $y^{(r)}(n)$. The Euclidean distance among observation $y(n)$ and its $r$-th neighbor is presented in Equation B.1. By adding a new dimension, the time series is reconstructed adding coordinate $(m+1)$ to every vector representing an observation $y(n)$, as represented by term $x(n+m T)$ in Equation B.2. Hence, this method evaluates the distance variation as new dimensions are added according to 
Equation B.3.

$$
\begin{gathered}
R_{m}^{2}(n, r)=\sum_{k=0}^{m-1}\left(x(n+k T)-x^{(r)}(n+k T)\right)^{2} \\
R_{m+1}^{2}(n, r)=R_{m}^{2}(n, r)+\left(x(n+m T)-x^{(r)}(n+m T)\right)^{2} \\
V_{n, r}=\sqrt{\frac{R_{m+1}^{2}(n, r)-R_{m}^{2}(n, r)}{R_{m}^{2}(n, r)}}=\frac{\left|x(n+m T)-x^{(n)}(n+m T)\right|}{R_{m}^{2}(n, r)}
\end{gathered}
$$

According to Kennel et al. (1992), if the distance variation $V_{n, r}$ is greater than a threshold $R_{t o l}$, then observations are considered as false neighbors. As discussed in (Kennel et al., 1992; Mello, 2009), an acceptable value for this threshold is $R_{t o l} \geq 10.0$.

In relation to the delay dimension, there are plenty of methods to estimate it, however, a comparison among them is out of the scope of this work. In this thesis, we studied the results presented by Fraser and Swinney (1986), who analyzed the Average Mutual Information (AMI) ${ }^{1}$ method to estimate this dimension. In summary, this method, widely adopted in Dynamical Systems and Chaos Theory area, works analyzing time series in different time delays. Afterwards, a curve is plotted considering the time delays and the first minimum is adopted as the dimension.

Aiming at better exemplifying the concepts of embedded and delay dimensions, consider a deterministic time series created by a Lorenz system (Alligood et al., 1997) with parameters $\sigma=10, \rho=28$ and $\beta=8 / 3$, which has chaotic behavior. Figure B. 1 shows 1,000 observations created by this Lorenz system.

The first step to unfold this time series is performed by estimating the delay dimension, which is calculated using the AMI method. By analyzing results presented in Figure B.2, we notice the estimated value for this dimension is 5 , once it is the first minimum located on the $x$-axis.

After estimating this dimension, FNN is applied on the time series to calculate the embedded dimension. By analyzing the FNN results presented in Figure B.3, we notice the false neighbor rate never reaches zero. In this situation, according to Kennel et al. (1992),

\footnotetext{
${ }^{1}$ Fraser and Swinney (1986) names his approach as Auto-Mutual Information, due to it is the Average Mutual Information applied on the time series itself.
} 


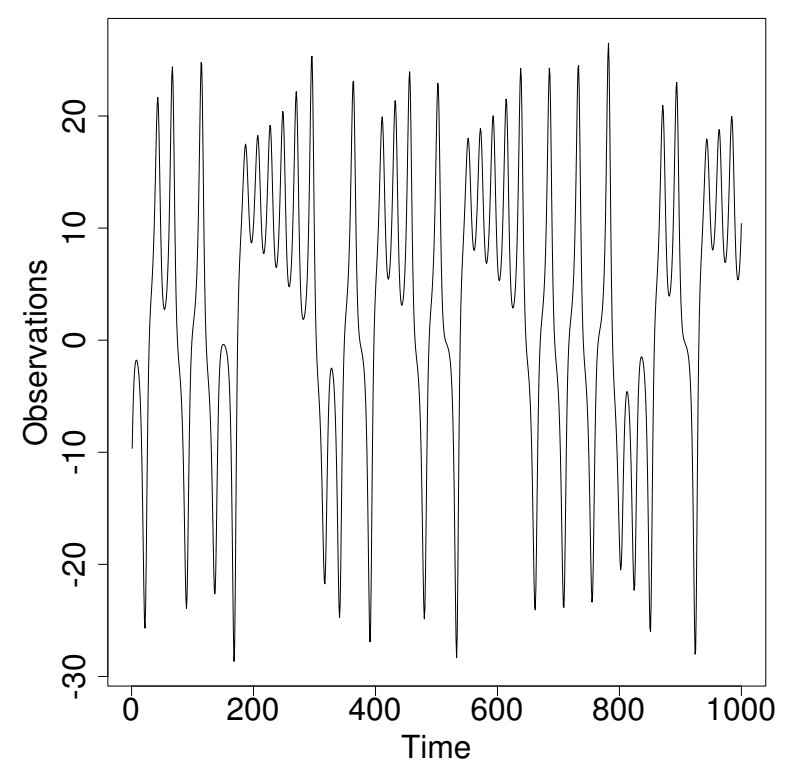

Figure B.1: Time series produced by the Lorenz system.

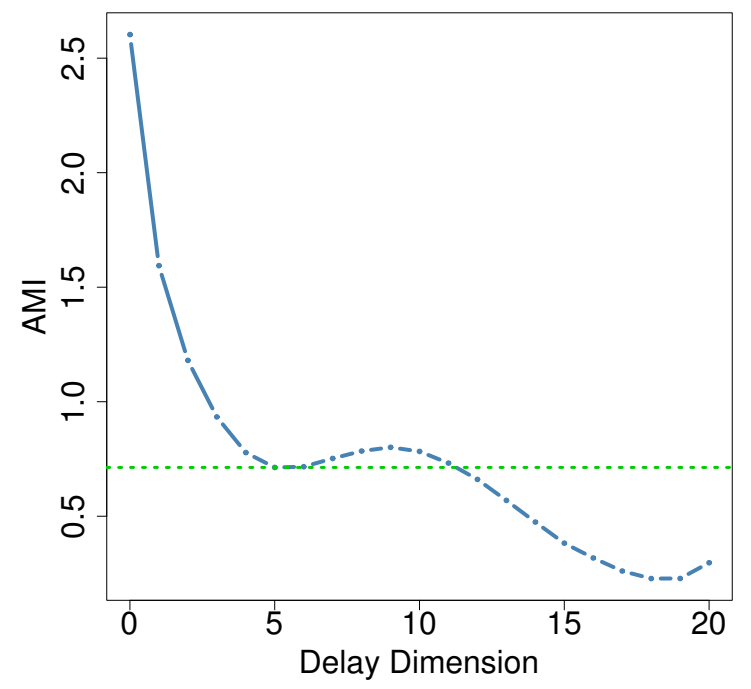

Figure B.2: Estimating the delay dimension using AMI.

the next value bellow $0.3(30 \%)$ is adopted as embedded dimension (Mello, 2009). Hence, for this plot, the embedded dimension is 3 .

Considering the values estimated by the AMI and FNN methods, the Lorenz time series is unfolded into a phase space with $m=3$ and $\tau=5$, i.e., in order to study a observation 


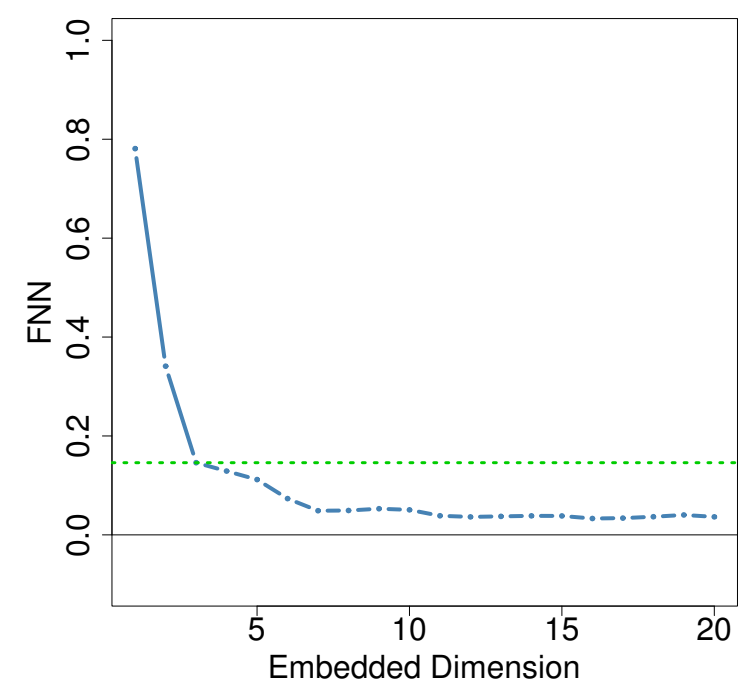

Figure B.3: Estimating the embedded dimension using FNN.

$x(t)$, it is also necessary to consider $\{x(t+5), x(t+10)\}$. A short sample of the unfolded time series is presented in Table B.1.

The unfolded time series, presented in Table B.1(b), was also plotted in Figure B.4. By analyzing this plot, we visualize the periodic orbits, illustrating the immersion theorem proposed by Takens (1980), which states time series can be better understood when its attractors are unfolded into the correct number of dimensions. 
Table B.1: (a) Time series produced by the Lorenz system; (b) Time series unfolded into the phase space with embedded and delay dimensions equal to $m=3$ and $\tau=5$, respectively.

(a)

\begin{tabular}{l}
\hline Dimension 1 \\
\hline \hline-9.6559617 \\
-6.9902085 \\
-4.9834927 \\
-3.5773619 \\
-2.6589215 \\
-2.1120568 \\
-1.8411753 \\
-1.7784935 \\
-1.8834828 \\
-2.1397586 \\
-2.5521791 \\
-3.1453527 \\
-3.9638112 \\
-5.0733551 \\
-6.5619076 \\
-8.5356685 \\
-11.100864 \\
-14.311700 \\
-18.056232 \\
-21.873802 \\
-24.819411 \\
\hline
\end{tabular}

(b)

\begin{tabular}{lll}
\hline Dimension 1 & Dimension 2 & Dimension 3 \\
\hline \hline-9.6559617 & -2.1120568 & -2.5521791 \\
-6.9902085 & -1.8411753 & -3.1453527 \\
-4.9834927 & -1.7784935 & -3.9638112 \\
-3.5773619 & -1.8834828 & -5.0733551 \\
-2.6589215 & -2.1397586 & -6.5619076 \\
-2.1120568 & -2.5521791 & -8.5356685 \\
-1.8411753 & -3.1453527 & -11.100864 \\
-1.7784935 & -3.9638112 & -14.3117 \\
-1.8834828 & -5.0733551 & -18.056232 \\
-2.1397586 & -6.5619076 & -21.873802 \\
-2.5521791 & -8.5356685 & -24.819411 \\
\hline
\end{tabular}




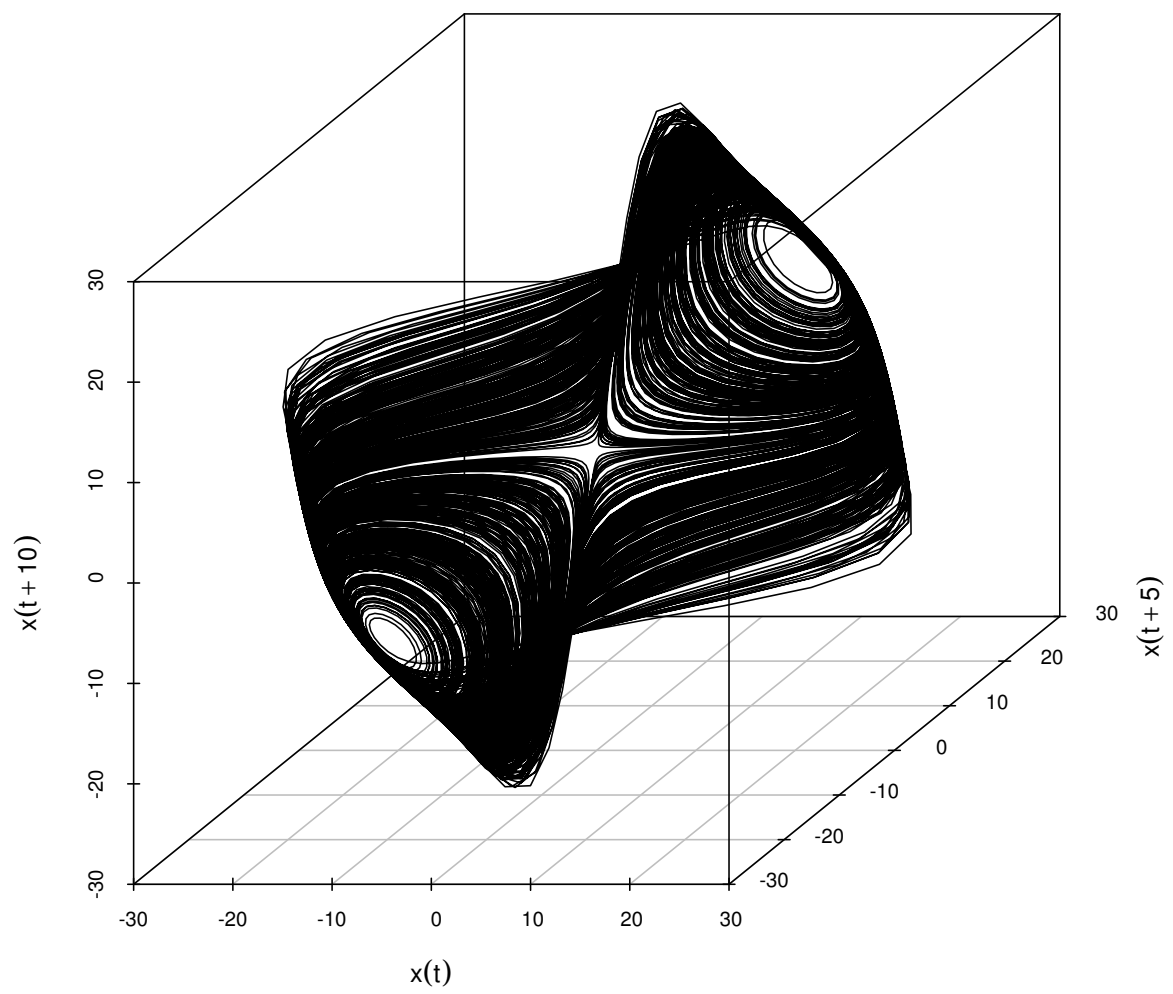

Figure B.4: Lorenz system: time series unfolded into phase space. 


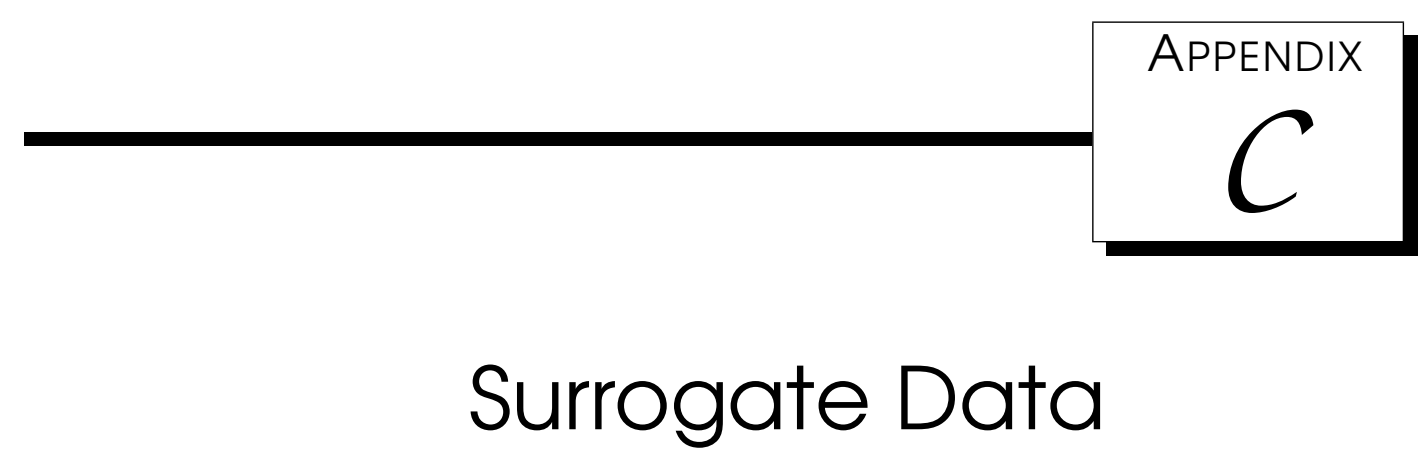

\section{C.1 Introduction}

Surrogate data methods (Theiler et al., 1992; Nakamura et al., 2006) are widely used to produce synthetic data respecting the same statistical properties as the original: such as mean, variance, autocorrelation function and power spectrum (Shoukat Choudhury et al., 2008). In that sense, surrogate data has been considered in two different scenarios: i) to analyze the properties of the time series generation process, i.e., the system that produced the series; and ii) in order to extend the data set size when few examples or observations are available.

In the first situation, comparing the synthetic against the original data, we can verify if both series were produced by the same process (Theiler et al., 1992). The most common property analyzed using such comparison is the linearity/nonlinearity (Theiler et al., 1992). For this purpose, Surrogate methods produce linear data, consequently, if the original data does not follow the same property as the surrogates, we can assume it does not come from that type of linear process (commonly either linearly filtered noise or a monotonic nonlinear transformation of linearly filtered noise). In the second situation, assuming there is not 
enough data to be modeled, one can produce more data using surrogate methods ${ }^{1}$. This can be used, for example, to produce synthetic data as an approach to increase the training sample size and, therefore, improve the estimated models. This is particularly interesting when dealing with machine learning techniques (Hirata et al., 2008; Shoukat Choudhury et al., 2008).

The most commonly considered surrogate data methods are based on the Fourier transform, such as the Fourier Transformed (FT) ${ }^{2}$ and Amplitude Adjusted Fourier Transformed (AAFT) methods proposed by Theiler et al. (1992). These methods basically apply the Fourier transform on the original data, obtaining amplitudes and phases, and substitute the original phases by uniform random phases. Afterwards, they apply the inverse Fourier transform to obtain the surrogate data. As this new data was generated using the Fourier transform, which assumes data periodicity, nonstationary characteristics are not represented in the surrogate. The main reason behind this is that by applying the Fourier transform on nonstationary time series, the difference between the first and last observations, caused by the trend, produces spurious high frequencies, as a consequence, inconsistent surrogates are produced (Nakamura et al., 2006; Nakamura and Small, 2005).

Aiming at overcoming this problem, we extended these two Fourier-based methods to, initially, decompose the time series into a set of components plus a residue. Every component contains similar behavior and the residue contains trends. Then, surrogate data is produced based on each individual component. Later on, all produced surrogates plus the original residue are added to compose the surrogate, which is indeed considered as synthetic data based on the original time series. To separate components, we consider the Empirical Mode Decomposition (EMD) method. The last component produced by EMD, i.e., the trend, contains nonstationary features. According to our experiments, we confirmed that our approach improves Theiler's methods to produce synthetic data to nonstationary time series.

\footnotetext{
${ }^{1}$ Of course, this new data does not contain any additional information, but it can still be advantageous for some nonlinear modeling routines.

${ }^{2}$ In this appendix, FT stands for the Theiler's Fourier Transformed method used to produce surrogates. On the other hand, the basic Fourier transform is referred to as $\mathcal{F}$.
} 


\section{C.2 Surrogate methods}

The study of surrogate data was introduced by Theiler et al. (1992), whose main objective was to analyze time series to confirm whether they belong to the same generation process. In general, this evaluation is performed in two straightforward steps. First, synthetic data is produced combining part of the original data properties and another specific generation process. This step is repeatedly performed to produce a set of surrogates. In the second step, discriminating statistics are computed to compare the original time series against all surrogates. Based on the computed values, one can verify the similarity among them, and, consequently, state whether or not they were created using the same process.

The discriminating statistics can be computed using different methods, such as the Grassberger-Procaccia (GP) correlation dimension (Grassberger and Procaccia, 1983), Autocorrelation Function (ACF) (Box et al., 1994), Spectral Density (SD) (Brockwell and Davis, 2002), Average Mutual Information (AMI) (Abarbanel, 1996) and Space-Time Separation Plot (STP) (Provanzale et al., 1992).

The results obtained by the discriminating statistics are then used to perform Statistical Hypothesis tests which assess the null hypothesis that the original and surrogate data are similar. For example, if we intend to test the null hypothesis that the original data is linear, we can produce surrogate data guaranteeing it will be linear. Thus, if the original and surrogate data have the same properties, we accept the null hypothesis, which simply states we failed to find evidence that the original data is not linear. However, if the null hypothesis is rejected then we have shown with some statistical confidence that the original data is not linear.

Initially, Theiler et al. (1992) proposed two methods to generate surrogate data widely studied and employed in several work (Maiwald et al., 2008; Small and Tse, 2003; Tohru and Kazuyuki, 5 25): the Fourier Transformed (FT) surrogate; and the Amplitude-Adjusted Fourier Transformed (AAFT) surrogate.

The FT method was designed to identify the nonlinear property in time series. This method defines as the null hypothesis that the analyzed time series is linear (Theiler et al., 1992; Maiwald et al., 2008). Hence, this method produces surrogates using a linear process. Afterwards, discriminating statistics are computed on the original and surrogates. 
Finally, if statistics are significantly different, the null hypothesis is rejected.

In order to better understand the FT method, consider a time series $x(t)=$ $\{x(1), x(2), \cdots, x(N)\}$ with length $N$. Let $X(f)$ be the coefficient produced by the Fourier transform $\mathcal{F}$ at frequency $f$ on $x(t)$, Equation C.1.

$$
X(f)=\mathcal{F}(x(t))=\int_{-\infty}^{\infty} x(t) \cdot e^{-i 2 \pi f t} d t
$$

The previous equation can also be rewritten in terms of its amplitude $A(f)$ and phase $\phi(f)$ as shown in Equation C.2.

$$
X(f)=A(f) \cdot e^{i \phi(f)}
$$

Then, the phases-randomized Fourier transform is obtained by rotating the phase at each frequency $f$ considering an independent random variable $\varphi$ which is uniformly chosen within the interval $[0,2 \pi)$ (Theiler et al., 1992), as shown in Equation C.3.

$$
\tilde{X}(f)=A(f) \cdot e^{i[\phi(f)+\varphi(f)]}
$$

Given the inherent linearity of the uniform distribution, this randomization creates surrogates with phases varying in a linear way. Finally, the surrogate data $y(t)$ is obtained by applying the Inverse Fourier transform $\mathcal{F}^{-1}$ (Equation C.4) (Theiler et al., 1992).

$$
y(t)=\mathcal{F}^{-1}(\tilde{X}(f))=\int_{-\infty}^{\infty} X(f) \cdot e^{i \varphi(f)} d f
$$

The second surrogate data method proposed by Theiler et al. is called the Amplitude Adjusted Fourier Transformed (AAFT) method. In this case, the null hypothesis assumes that besides the time series dynamics are linear, observations may be influenced by a nonlinear static transform (Theiler et al., 1992). According to authors, most conventional methods used to estimate nonlinearity indicate that a given time series is nonlinear, but they do not provide further information to conclude if the nonlinearity comes from the time series dynamics or from the amplitude distribution (Theiler et al., 1992). On the other hand, by using AFFT, surrogates are produced respecting the same amplitude distribution of the original time series and presenting similar ACF, but not equal, once there is an 
adjustment on the amplitude (Theiler et al., 1992; Lucio et al., 2012). Aiming at improving AAFT to produce surrogates that preserve both amplitude distribution and ACF, Schreiber and Schmitz (1996) proposed a new method called Iterative Amplitude Adjusted Fourier Transformed (IAAFT). However, this method is not considered in our comparative study.

The AAFT method (Theiler et al., 1992) receives the original time series $x(t)$ and then computes a rank for each observation. Then, the computed ranks are used to sort the $x(t)$ observations in an increasing order, returning a new series $x_{r}(t)$. Next, AAFT generates a new time series $y(t)$ using a Gaussian process. This new time series is reordered so that the ranks agree with the $x_{r}(t)$ ranks. After that, the FT method is applied on $y(t)$ generating a new series $y^{\prime}(t)$. Finally, AAFT produces the surrogate data $x_{s}(t)$ for $x(t)$ by reordering the observations in $x(t)$ in a way that its ranks agree with the $y^{\prime}(t)$ ranks. (Theiler et al., 1992).

The main problem faced by these methods is related to the stationarity restriction imposed by the Fourier transform. Theiler's FT and AAFT methods cannot create surrogate data sufficiently similar to time series characterized by nonstationary behavior. In such situation, the surrogate produced by these methods is affected by the amplitude variation in the Fourier transform, producing surrogates completely different from the original time series (Nakamura et al., 2006; Nakamura and Small, 2005).

Another surrogate method called Small Shuffle Surrogate (SSS) was proposed by Nakamura and Small(Nakamura and Small, 2005). To generate surrogate data, this method performs the following steps: i) the original time series $x(t)$ is analyzed and the indices of its observations are stored in a list $i(t)$; ii) a new index list is created by considering equation $i^{\prime}(t)=i(t)+A \cdot g(t)$, in which $A$ represents an amplitude and $g(t)$ is a sequence of Gaussian random numbers. In this equation, the amplitude $A$ is responsible for defining the scale of changes in the index list $i(t)$; iii) list $i^{\prime}(t)$ is sorted and stored in a new list $\hat{i}(t)$; iv) finally, a surrogate $s(t)$ is obtained by selecting values of the original time series $x(t)$ according to the new indexes $\hat{i}(t)$, i.e., $s(t)=x(\hat{i}(t))$.

According to the authors, this method can be used to investigate irregular fluctuations in time series, once it destroys local structures or correlations and keeps the global behavior, such as trend. Hence, the null hypothesis addressed by this new method states that if the irregular fluctuation present in time series is not random, then there is some kind of dynamics, i.e., it is possible to notice the presence of deterministic behavior in obser- 
vations even if they are influenced by stochasticity and characterized by long-term trends and periodicity.

The main limitation of the SSS method comes from the difficulty to indicate if data are linear or nonlinear, because both behaviors are characterized by some dynamics (Nakamura et al., 2006). Aiming at solving this limitation, the same authors presented a new method called Truncated Fourier Transform Surrogate (TFTS), which produces surrogates by randomizing phases only in the high-frequency domain. For this, they define a threshold $f_{\varepsilon}$ to determine whether phases may change or not, i.e., if phases are characterized by frequencies higher than $f_{\varepsilon}$, then they are randomized; otherwise, they remain the same. Since high-frequency phases are randomized, the nonlinearity present in irregular fluctuations is destroyed, whereas the global behavior is preserved by the untouched phases. Hence, the null hypothesis addressed by the TFTS method states that irregular fluctuations are generated by stationary linear systems (Nakamura et al., 2006).

The TFTS method was later considered by Lucio et al. (2012), which presented two new techniques named AAFT $\mathrm{T}_{\mathrm{TD}}$ and $\mathrm{IAAFT}_{\mathrm{TD}}$. Similarly to our approach, the authors designed these techniques to preserve the global nonstationarity present in time series. In summary, the techniques consist of detrending and retrending the time series, applying the TFTS and AAFT (or IAAFT) method to generate the surrogate data. These last three methods emphasize the importance of producing surrogate data considering nonstationary behavior in time series as approached in this work.

The main problem with the TFTS method, and consequently with the methods based on it, is the need for setting a value for parameter $f_{\varepsilon}$, which determines the frequencies to be randomized. In summary, when this parameter assumes low values, most of the phases will be randomized, producing surrogates very similar to the traditional FT method. On the other hand, high values mean the surrogate will be very close to the original data.

Concluding, the nonstationary problem faced by the traditional Theiler FT and AAFT methods and the restriction imposed by the parameter $f_{\varepsilon}$ to the TFTS method have motivated the development of two new methods presented in the following section. 


\section{C.3 Proposed surrogate methods}

The surrogate methods presented in this appendix were designed to solve the problem faced by Theiler's Fourier Transformed (FT) and Amplitude Adjusted Fourier Transformed (AAFT) methods when analyzing nonlinear time series. As previously presented, by applying these Fourier-based methods on time series with trends, the produced observations are influenced by spurious high frequencies, which affect the general behavior of surrogates. In order to overcome this drawback, we initially decompose time series into a set of monocomponents plus a residue, which represents the time series trend. Afterwards, we apply traditional surrogate methods on every monocomponent, producing a set of monocomponent surrogates. Those individual surrogates are combined to produce a single surrogate data, which is finally retrended by adding the residue obtained in the first step. These detrending and retrending steps allow to preserve the global nonstationarity in the surrogate series.

This decomposition step was performed by using of the Empirical Mode Decomposition (EMD) method (Huang et al., 1998), which reduces the time series into monocomponents, also called Intrinsic Mode Functions (IMFs), revealing important information embedded in the original series (Huang et al., 1998). The most important advantage of using EMD is the possibility of decomposing time series irrespective of its generation processes, i.e., the decomposition proccess is not affected by the nonlinearity, nonstationarity, and/or stochasticity present in time series.

The key point to perform this decomposition is the sifting process, which initially identifies local minima and maxima values for observations along time. Afterwards, these extrema are connected through the cubic spline method and, thus, the upper and lower envelopes are defined, which must cover all values (Huang et al., 1998). Then, the mean $m_{1}(t)$ of these envelopes is calculated and the first monocomponent candidate $c_{1}(t)$ is obtained by using Equation C.5, in which $x(t)$ represents the analyzed time series.

$$
c_{1}(t)=x(t)-m_{1}(t)
$$

Then, the first monocomponent candidate $c_{1}(t)$ is used in place of the original data and the sifting process is repeated $k$ times, producing $c_{1 k}(t)=c_{1(k-1)}(t)-m_{1 k}(t)$. This process 
continues until $c_{1 k}(t)$ satisfies the IMF monocomponent definition (Huang et al., 1998): i) the number of extrema and the number of zero-crossings must be either equal or differ at most by one; or ii) at any point, the mean value of the envelope defined by the local maxima and the envelope defined by the local minima is zero. After retrieving the first monocomponent that satisfies the IMF definition, i.e., $c_{1 k}(t)$, the first IMF monocomponent is obtained according to Equation C.6.

$$
h_{1}(t)=c_{1 k}(t)
$$

Then, this first IMF is removed from data, i.e., $x(t)-h_{1}(t)$, and the resultant series is again analyzed by the whole process, producing further IMFs until reaching a stop criterion. This criterion is defined when the last component $h_{n}(t)$ becomes a monotonic function, avoiding the extraction of further components. Hence, this last component is referred to as final residue $r_{M}(t)$ (Huang et al., 1998). In summary, according to EMD, a time series $x(t)$ is composed of a set of monocomponents plus a residue as presented in Equation C.7, in which $M$ represents the number of monocomponents obtained from time series $x(t)$.

$$
x(t)=\sum_{m=1}^{M-1} h_{m}(t)+r_{M}(t)
$$

The decomposition step in our method permits detrending the time series, by the residue extraction, before applying any surrogate method. Hence, as next step, our method executes the Theiler's FT method on all decomposed monocomponents $\left\{h_{m}(t)\right\}$, except on residue $r_{M}(t)$, producing a set of monocomponent surrogates.

In the last step, all monocomponent surrogates are summed to form a single surrogate data. Finally, the global trend of the original time series is combined to this single surrogate by adding the residue. In summary, this new adapted method, called EMD-FT, is defined by Equation C.8, in which $y(t)$ is the surrogate data, $X_{m, k}(f)$ represents the coefficients obtained applying the Discrete Fourier transform on the $m$-th monocomponent, and $\varphi(f)$ represents the values obtained with the phase randomization.

$$
y(t)=\sum_{m=1}^{M-1} \sum_{n=1}^{N}\left(\frac{1}{N} \sum_{k=1}^{N-1} X_{m, k}(f) \cdot e^{i \varphi(f)}\right)+r_{M}(t)
$$

On the other hand, the adapted EMD-AAFT method was created using the exactly the 
same steps previously presented, however the AAFT method was adopted to produce surrogate data for every monocomponent instead.

The most important contribution of the proposed methods is the possibility of removing nonstationary influences during the decomposition of the original time series. After decomposition, every monocomponent contains simpler behavior which is better represented using sinusoidal functions. Thus, when Theiler's surrogate data methods are applied on every monocomponent to produce surrogates following the singular monocomponent behavior. Surrogate methods are not applied on the residue, which represents the original time series trend. Consequently, by using FT and AAFT in our methods, one can test the linearity in stationary or nonstationary time series, what was not possible using Theiler's methods directly on the original time series.

In order to evaluate the proposed methods, in the next section, we analytically demonstrate that surrogates produced by the original FT and AAFT methods are similar to the proposed EMD-FT and EMD-AAFT methods, even when there is a trend embedded in the time series.

\section{C.4 Analyzing the proposed surrogate methods}

Aiming at investigating the efficiency of the proposed methods, we analyzed the surrogate data produced by FT and EMD-FT methods. The main objective of this analysis is to assure that the adapted method produces surrogates with the same behavior as the traditional one and, consequently, the same null hypothesis can be used by both methods, even when there is nonstationary behavior in the data.

It is important to highlight that we used the EMD-FT method because it is more intuitive, but the same results can be extended to the EMD-AAFT method. In order to proceed with this analysis, we first present a theorem which states that the surrogate data produced by both methods are exactly the same, even when there is trend embedded in the time series. By proving this theorem we confirm the same hypothesis test used by the Theiler's FT method can be adopted for the EMD-FT method. In this sense, we can formally define our hypothesis: 
Theorem: If the trend can be linearly separable from nonlinear time series, then Theiler's FT and the proposed EMD-FT method produce the same surrogate data.

Proof: To validate this theorem, we need to prove that the surrogate data produced by both methods are exactly the same to linear and nonlinear time series. However, before proceeding with this analysis, we postulate the the trend can somehow be detached from time series.

Therefore, considering this postulate, we can rewrite a nonlinear time series as $x(t)=$ $z(t)+r_{M}(t)$, in which $z(t)$ represents the time series observations and $r_{M}(t)$ is the trend. We used $r_{M}(t)$ to represent the trend just to keep the same pattern used to describe the Empirical Mode Decomposition (EMD) method. Hence, in the first part of our proof, after applying Theiler's FT surrogate method on the observations of $z(t)$, we obtain the surrogate $y(t)^{F T}$.

$$
y(t)^{F T}=F T(z(t))+r_{M}(t)
$$

Considering the definition of Fourier transform (Equation C.1), we can rewrite the previous equation as:

$$
y(t)^{\mathrm{FT}}=\sum_{n=1}^{N}\left(\frac{1}{n} \sum_{k=1}^{N} X_{k}(f) \cdot e^{i \varphi(f)}\right)+r_{M}(t)
$$

The second part of our proof is, initially, obtained by applying the EMD method on the same nonlinear time series $x(t)$, which returns a set of monocomponents $\left\{h_{m}(t)\right\}$ and a residue $r_{M}(t)$. By definition, the residue $r_{M}(t)$ represents the time series trend, once the EMD method was previously proved to be nonlinear and useful to retrieve the trend from time series (Tsakalozos et al., 2012). Afterwards, Theiler's FT method is also used to generate surrogates, but Equation C. 1 may be individually applied on every monocomponent $h_{m}(t)$, resulting in the surrogate $y(t)^{E M D-F T}$. 


$$
\begin{aligned}
\operatorname{EMD}(x(t)) & =h_{1}(t)+\cdots+h_{m}(t)+r_{M}(t) \\
y(t)^{\mathrm{EMD}-\mathrm{FT}} & =F T\left(h_{1}(t)\right)+\cdots+F T\left(h_{m}(t)\right)+r_{M}(t) \\
& =\sum_{n=1}^{N}\left(\frac{1}{n} \sum_{k=1}^{N} X_{1, k}(f) \cdot e^{i \varphi(f)}\right)+\cdots+\sum_{n=1}^{N}\left(\frac{1}{n} \sum_{k=1}^{N} X_{m, k}(t) \cdot e^{i \varphi(f)}\right)+r_{M}(t)
\end{aligned}
$$

An important step of our proof is stated by assuming the phase randomization is performed only once for all monocomponents $h_{m}(t)$, i.e., all monocomponents were randomized considering the same sequence of values. Hence, the $y(t)^{E M D-F T}$ surrogate can be rewritten evidencing the randomized phase according to Equation C.12.

$$
\begin{aligned}
y(t)^{\mathrm{EMD}-\mathrm{FT}} & =\left[\sum_{n=1}^{N}\left(\frac{1}{n} \sum_{k=1}^{N} X_{1, k}(t)\right)+\cdots+\sum_{n=1}^{N}\left(\frac{1}{n} \sum_{k=1}^{N} X_{m, k}(t)\right)\right] \cdot e^{i \varphi(f)}+r_{M}(t) \\
y(t)^{\mathrm{EMD}-\mathrm{FT}} & =\left[\sum_{m=1}^{M-1} \sum_{n=1}^{N}\left(\frac{1}{n} \sum_{k=1}^{N} X_{m, k}(t)\right)\right] \cdot e^{i \varphi(f)}+r_{M}(t)
\end{aligned}
$$

Finally, in order to prove the theorem stated in this section, we need to evaluate the relation $y(t)^{F T}=y(t)^{E M D-F T}$, i.e., observations generated by both methods are equal.

$$
\begin{aligned}
y(t)^{F T} & =y(t)^{E M D-F T} \\
\sum_{n=1}^{N}\left(\frac{1}{n} \sum_{k=1}^{N} X_{k}(f) \cdot e^{i \varphi(f)}\right)+r_{M}(t) & =\left[\sum_{m=1}^{M-1} \sum_{n=1}^{N}\left(\frac{1}{n} \sum_{k=1}^{N} X_{m, k}(t)\right)\right] \cdot e^{i \varphi(f)}+r_{M}(t)
\end{aligned}
$$

By subtracting $r_{M}(t)$ from both sides of the Equation C.12, we obtain the final equality (Equation C.14) and prove the theorem.

$$
\sum_{n=1}^{N}\left(\frac{1}{n} \sum_{k=1}^{N} X_{k}(f) \cdot e^{i \varphi(f)}\right)=\left[\sum_{m=1}^{M-1} \sum_{n=1}^{N}\left(\frac{1}{n} \sum_{k=1}^{N} X_{m, k}(t)\right)\right] \cdot e^{i \varphi(f)}
$$


Finally, we divide both equation sides by $e^{i \varphi(f)}$ :

$$
\begin{aligned}
{\left[\sum_{n=1}^{N}\left(\frac{1}{n} \sum_{k=1}^{N} X_{k}(f)\right)\right] \cdot e^{i \varphi(f)} } & =\left[\sum_{m=1}^{M-1} \sum_{n=1}^{N}\left(\frac{1}{n} \sum_{k=1}^{N} X_{m, k}(t)\right)\right] \cdot e^{i \varphi(f)} \\
\sum_{n=1}^{N}\left(\frac{1}{n} \sum_{k=1}^{N} X_{k}(f)\right) & =\sum_{m=1}^{M-1} \sum_{n=1}^{N}\left(\frac{1}{n} \sum_{k=1}^{N} X_{m, k}(t)\right)
\end{aligned}
$$

This equality proves that the sum of amplitudes, obtained by applying Theiler's FT on EMD decomposed monocomponents, is equal to the amplitude obtained using Theiler's FT surrogate method directly on the time series.

Therefore, we confirm the new method supports the same null hypothesis as the original methods (Theiler's FT and AAFT), but without any interference of the nonstationary behavior, once the nonlinear EMD method permits treating the trend as a separated component.

In the following section, we present the experimental setup to evaluate the proposed methods.

\section{C.5 Experimental results}

In order to evaluate the proposed methods, we analyzed two sets of time series ${ }^{3}$. The first one was composed of three synthetic time series created by adding a trend to a sine function (Figure C.1(a)), a white noise process (Figure C.1(b)), and an autoregressive process (Figure C.1(c)). The autoregressive (AR) process used in these experiments was generated considering a model of order one and a Normal distribution $N\left(0, \sigma^{2}\right)$. This Normal distribution was also used to create the white noise time series presented in Figure C.1(b).

The second set was composed of three real-world time series. The fist series is illustrated in Figure C.1(d), which corresponds to a collection of yearly average global temperatures (Shumway and Stoffer, 2006). Figure C.1(e) presents the second time series that corresponds to atmospheric concentrations of CO2 (Cleveland, 1993). Finally, Figure C.1(f)

\footnotetext{
${ }^{3}$ The time series used in this experiments, as well as the source code, are available in: http://www.icmc. usp.br/ rios/surrogate/
} 
represents the Dow Jones Utilities Index commonly considered in stock market analysis (Brockwell and Davis, 2002).

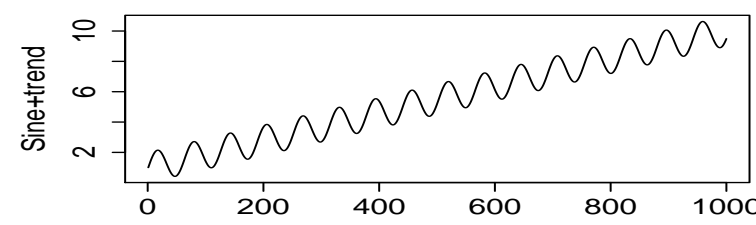

(a)
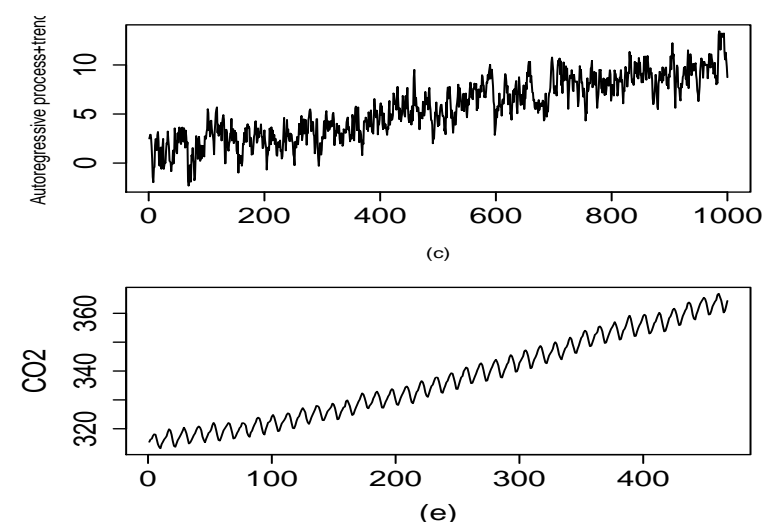

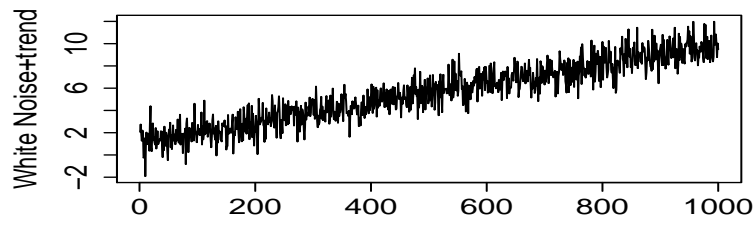

(b)
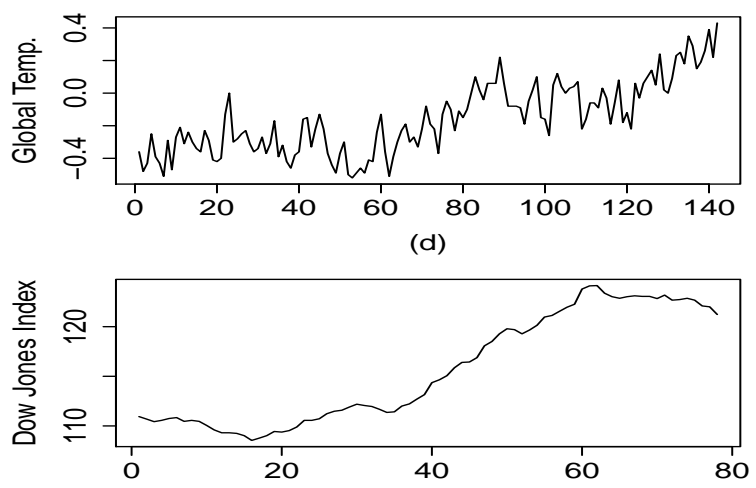

(f)

Figure C.1: Time series used to evaluate the proposed methods: (a) A sine function combined with a trend; (b) A white noise process combined with a trend; (c) Autoregressive process combined with a trend; (d) Global Temperature (Shumway and Stoffer, 2006); (e) Atmospheric concentrations of CO2 (Cleveland, 1993); (f) Dow Jones Utilities Index (Brockwell and Davis, 2002).

The evaluation process was performed by analyzing every time series and producing 99 surrogates using the methods FT, AAFT, EMD-FT, and EMD-AAFT. Then, the original and surrogate time series were evaluated considering three types of analyses: i) visual inspection of time series plots; ii) visual inspection of plots produced by the Autocorrelation Function (ACF) (Provanzale et al., 1992) and the Average Mutual Information (AMI) (Abarbanel, 1996; Nakamura and Small, 2005; Nakamura et al., 2006); iii) hypothesis test considering the discriminating statistics obtained using AMI.

The Autocorrelation Function allows to identify temporal correlations present in series at different lags in time, depicting the difference among the methods. Formally, the Autocorrelation function $\hat{\rho}(h)$ of a time series $x(t)$ is obtained by computing the autocovariances of $x(t)$ and its time-shifted version $x(t+h)$ as defined in Equation C.16, in which $E[\cdot]$ is the expected value of the expression, and $\mu$ and $\sigma^{2}$ are the variance and mean of $x(t)$, 
respectively.

$$
\hat{\rho}(h)=\frac{E[(x(t)-\mu)(x(t+h)-\mu)]}{\sigma^{2}}
$$

In summary, ACF allows to analyze the similarity among time series observations. By considering it as discriminating function, we can evaluate whether the similarity among the original time series observations agrees with the similarities of surrogates or not.

The Average Mutual Information (AMI) can be considered a nonlinear version of ACF, which helps to determine the dependence between past and future observations (Abarbanel, 1996; Nakamura and Small, 2005; Nakamura et al., 2006). Equation C.17 defines AMI, in which $p(x(t))$ represents the marginal probability distribution function of $x(t)$ and $p(x(t), x(t+h))$ is the joint probability distribution function of $x(t)$ and $x(t+h)$, having $h$ as the time lag. In all experiments, the time lag as varied within interval $[1,20]$ and sixteen bins where used to discretize data and estimate the probabilities.

$$
I(h)=\sum_{x} p(x(t), x(t+h)) \log \left(\frac{p(x(t), x(t+h))}{p(x(t)) p((t+h))}\right)
$$

\section{C.5.1 Synthetic Time Series}

In the first synthetic experiment, we analyzed a time series created by the combination of a sine function and a linear sequence of observations, added to simulate a trend behavior. This time series (red continuous line) and the surrogates (dashed lines) generated by FT, AAFT, EMD-FT, and EMD-AAFT are illustrated in Figures C.2(a), C.2(d), C.2(g), and C.2(j), respectively. By visually inspecting these plots, we observe EMD-FT produced surrogates whose behavior is very similar to the original series. Although the surrogates produced by the EMD-AAFT method are also close to the original time series, we notice the presence of small noise changing the expected behavior.

These conclusions were also drawn by analyzing the discriminating statistics. According to ACF (Figures C.2(b), C.2(e), C.2(h), and C.2(k)) and AMI (Figures C.2(c), C.2(f), C.2(i), and C.2(1)) plots, the original data (red continuous line) only falls within the surrogate distribution (dashed lines) produced by the EMD-FT method.

In the second synthetic experiment, we analyzed a time series (Figure C.3(a)) created 

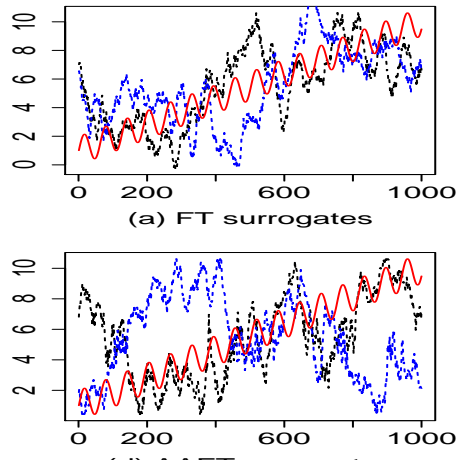

(d) AAFT surrogates
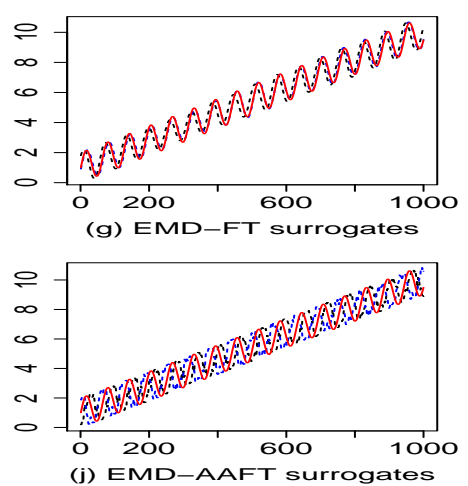

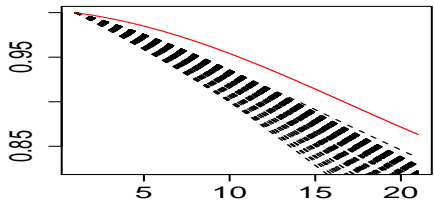

(b) $\mathrm{FT}$ ACF

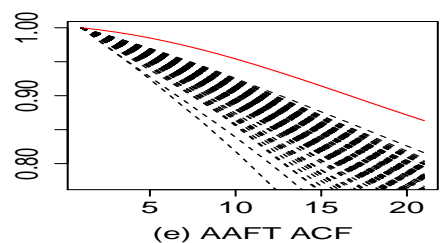

(e) AAFT ACF
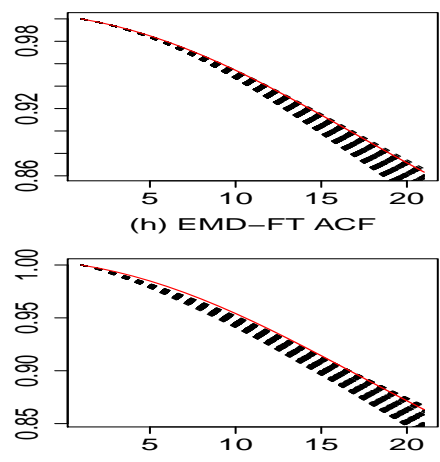

(k) EMD-AAFT ACF

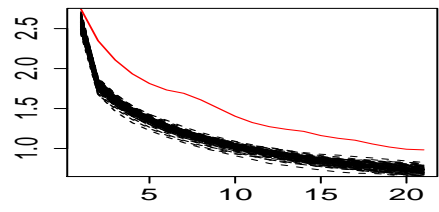

(c) FT AMI
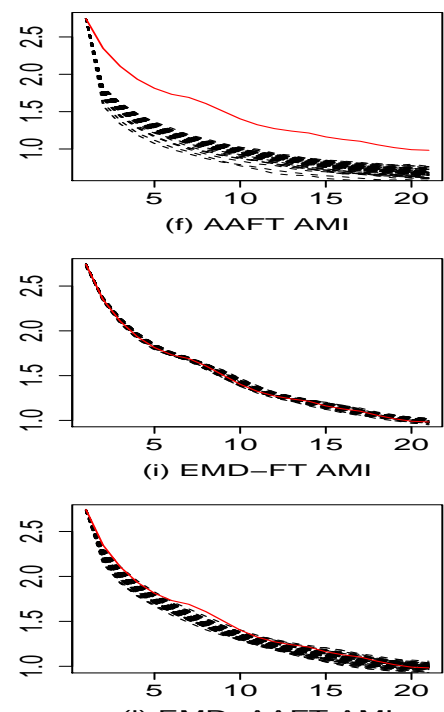

(I) EMD-AAFT AMI

Figure C.2: Surrogates generated from the synthetic time series created by the combination of trend and sine function. The left side presents the original time series (red continuous line) and its surrogates (dashed lines). In the middle and right side, it is presented the ACF and AMI plots.

by the combination of a white noise process and a linear sequence of observations used to simulate a trend behavior.

By analyzing the plots in Figure C.3, we confirm the proposed methods produce surrogates, which behavior is closer to the original time series than the Theiler's FT and AAFT methods. In case of EMD-FT and EMD-AAFT, we observe no significative differences.

The last synthetic time series was created combining an autoregressive process to a linear sequence of observations. Observing Figure C.4, the proposed methods generated surrogates more consistent with the original time series. We highlight there is no significative difference between EMD-FT and EMD-AAFT when visually inspecting the time series plots.

In the next section, we present the results obtained when analyzing real-world time series. 

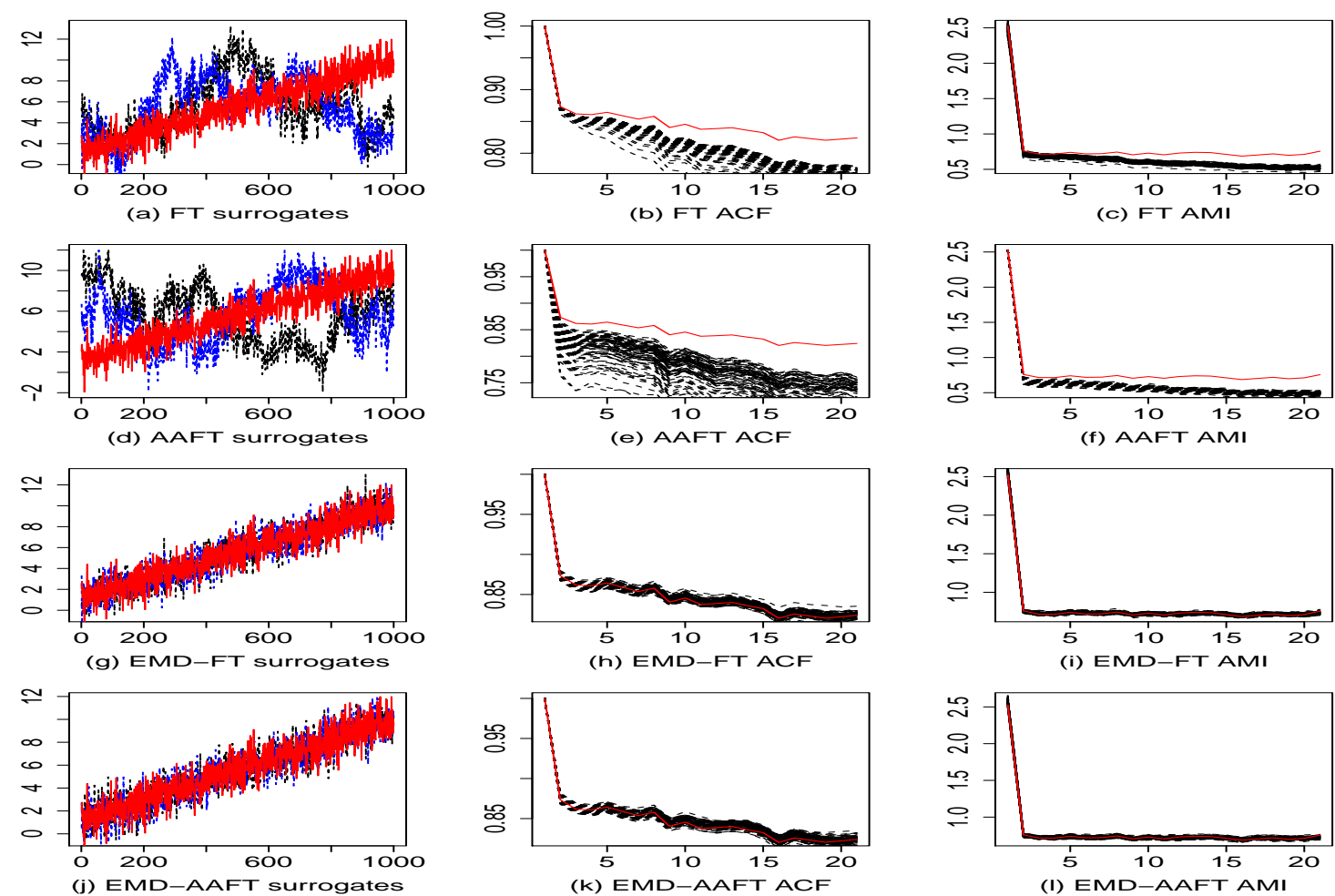

(k) EMD-AAFT ACF

(I) EMD-AAFT AMI

Figure C.3: Surrogates generated from the synthetic time series created by the combination of trend and white noise process. The left side presents the original time series (red continuous line) and its surrogates (dashed lines). In the middle and right side, it is presented the ACF and AMI plots.

\section{C.5.2 Real-World Time Series}

The first time series analyzed in this section is presented in Figure C.5(a). This time series was studied in (Shumway and Stoffer, 2006) and it is composed of yearly average values of global temperatures.

By analyzing this series, we realized observations follow a trend, i.e. the mean temperature increases over time, characterizing the time series as nonstationary. Nevertheless, the surrogates created by Theiler's FT and AAFT cannot represent such trend. On the other hand, the EMD-FT and EMD-AAFT methods produced surrogates considering the nonstationarity of the original time series. Analyzing only the AMI plots, we conclude all methods produced surrogates, which are compatible with the original time series, once they are within the surrogate distribution. However, in this situation, the ACF plot clearly indicates Theiler's FT and AAFT surrogates are different from the original time series, what is evident 

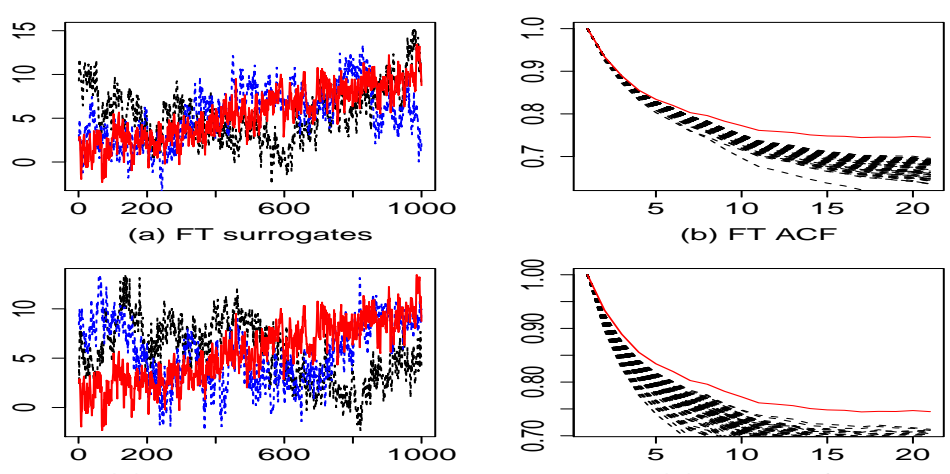

(d) AAFT surrogates
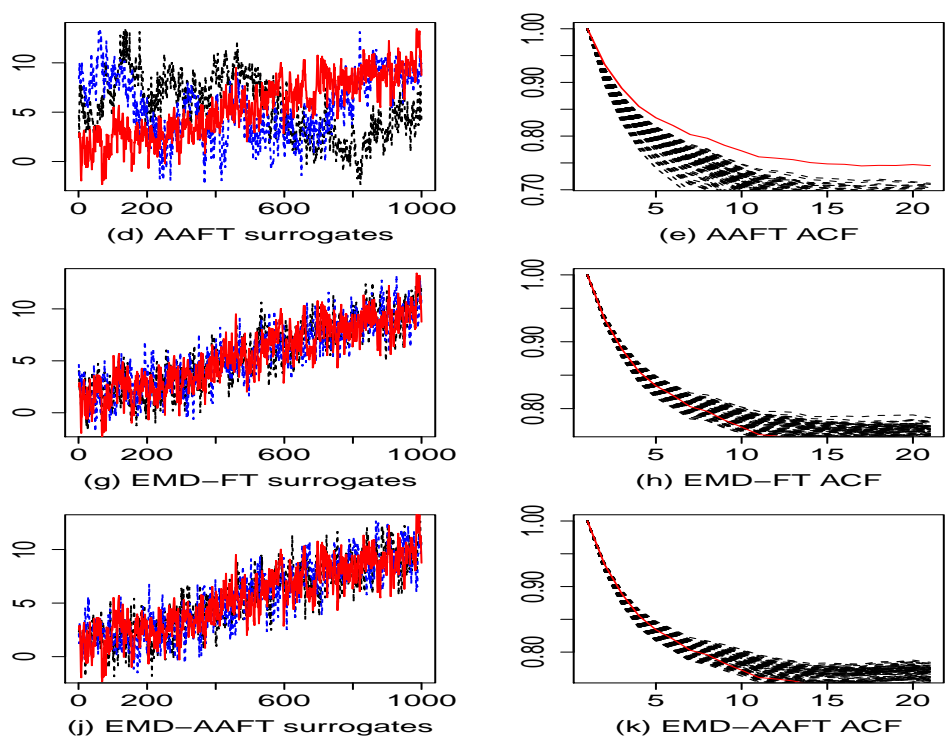

(e) AAFT ACF

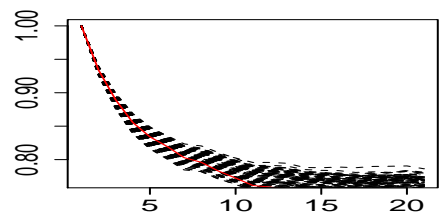

(h) EMD-FT ACF

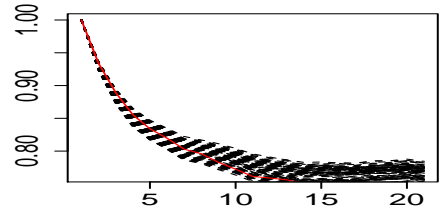

(k) EMD-AAFT ACF

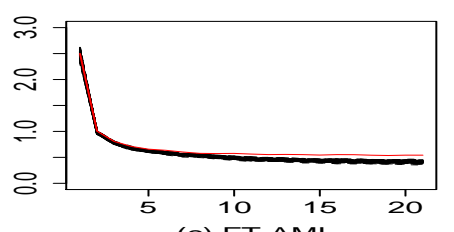

(c) FT AMI
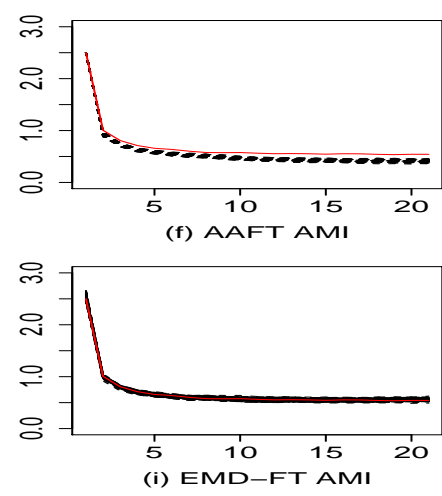

(I) EMD-AAFT AMI

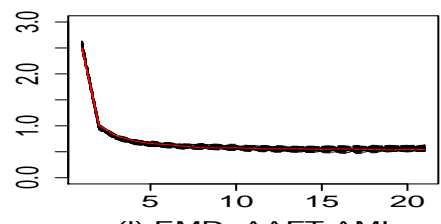

Figure C.4: Surrogates generated from the synthetic time series created by an autoregressive noise. The left side presents the original time series (red continuous line) and its surrogates (dashed lines). In the middle and right side, it is presented the ACF and AMI plots.

by plots on left side of Figure C.5.

The second real-world time series considered in this study is composed of atmospheric concentrations of $\mathrm{CO} 2$ and has a similar behavior to the synthetic series presented in Figure C.2. This series is characterized by some trend and cyclical behavior. In this scenario, the best surrogates were generated using EMD-FT and EMD-AAFT, as expected due to presence of trend. This is also confirmed by the ACF and AMI plots. In this situation, there is no significative difference between the EMD-FT and EMD-AAFT surrogates.

Finally, the last experiment was performed on the Dow Jones Utilities Index, which was recorded from August $28^{\text {th }}$ to December $18^{\text {th }}, 1972^{4}$ (Brockwell and Davis, 2002). This time series (Figure C.7) has a trend behavior as well, benefiting the EMD-FT and EMD-AAFT methods. This conclusion is also evident in the ACF plots. However, according to the AMI plots, the only ineffective method was Theiler's AAFT. In such situation, EMD-FT and

\footnotetext{
${ }^{4}$ Although there are most current observations for this dataset, we used this period due to its adoption in several papers and textbooks on time series analysis.
} 


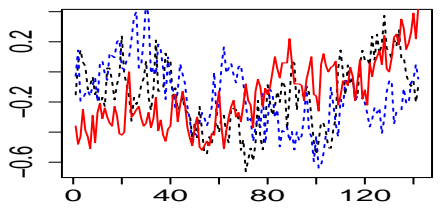

(a) FT surrogates

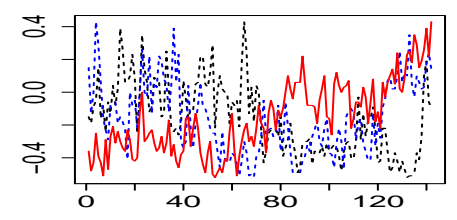

(d) AAFT surrogate

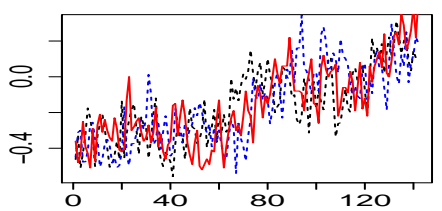

(g) EMD-FT surrogate

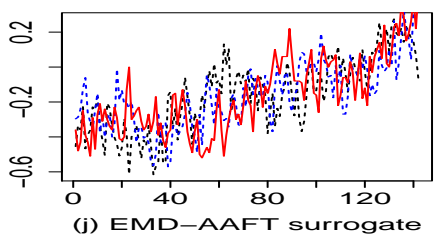

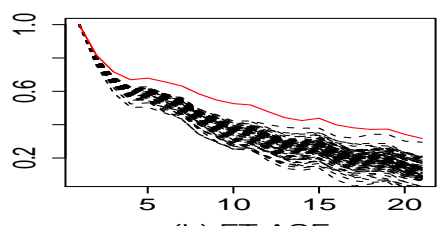

(b) FT ACF
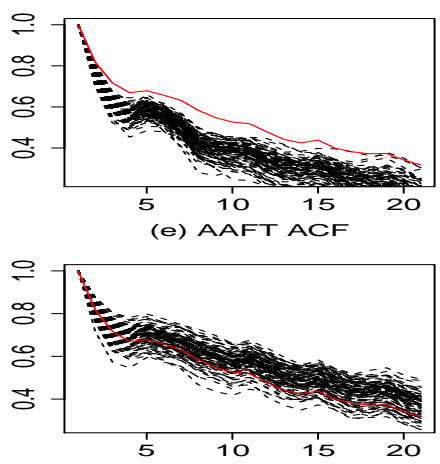

(h) EMD-FT ACF

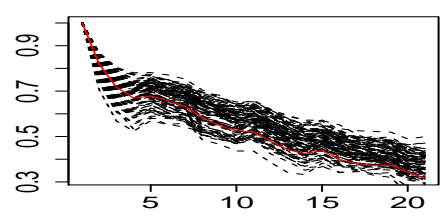

(k) EMD-AAFT ACF

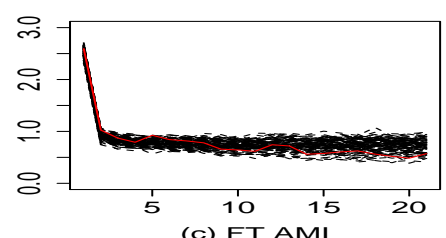

(c) FT AMI
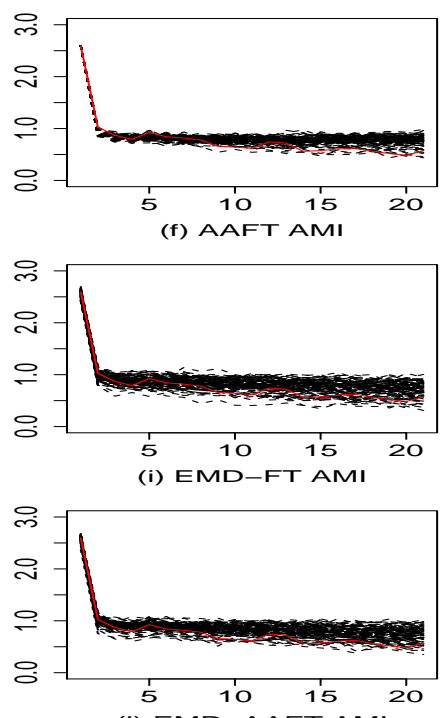

(1) EMD-AAFT AMI

Figure C.5: Surrogates generated from the real-world time series composed of average values, yearly, collected of global temperatures. The left side presents the original time series (red continuous line) and its surrogates (dashed lines). In the middle and right side, it is presented the ACF and AMI plots.

EMD-AAFT surrogates have similar behavior.

Finally, we also applied a hypothesis test on the discriminating statistics produced by the Average Mutual Information. Thus, we applied hypothesis tests to compare the original time series against every surrogate produced by all methods. Then, we computed the average p-value $\mu_{p-v a l u e}$ for every method. At last, the following hypothesis test was applied to compare the methods, which considers a significance level of 0.01 for the one tailed test.

$$
\begin{cases}H_{0}: & \mu_{\mathrm{p}-\text { value }} \geq 0.01 \\ H_{a}: & \mu_{\mathrm{p} \text {-value }}<0.01\end{cases}
$$

This test accepts the null hypothesis when the average p-value is greater than 0.01 , otherwise, we accept the alternative hypothesis, which allows us to infer the surrogate and original time series were not produced using the same generation process. The obtained 


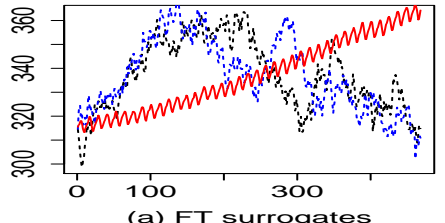

(a) FT surrogates

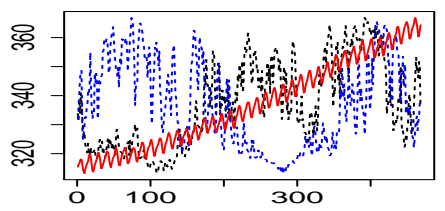

(d) AAFT surrogate

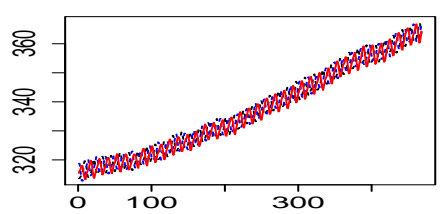

(g) EMD-FT surrogate

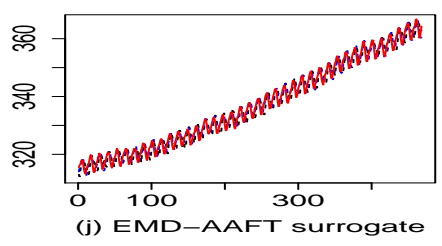

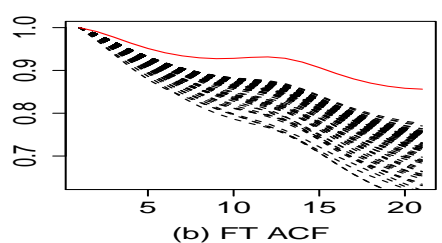

b) $\mathrm{ACF}$
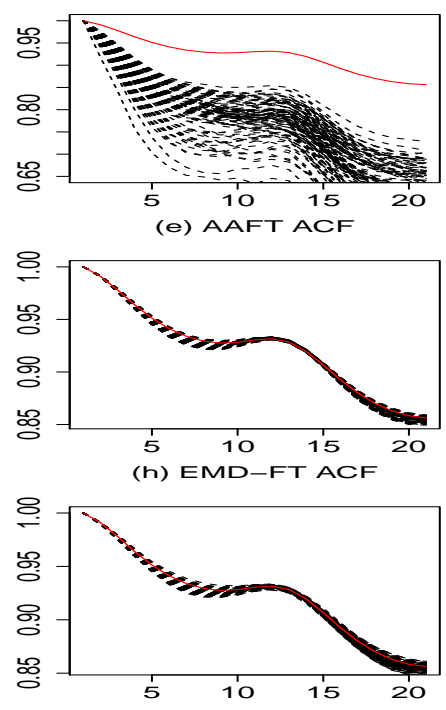

(k) EMD-AAFT ACF

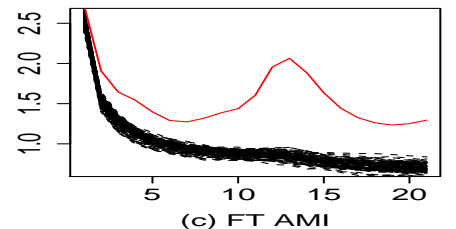

(C) FT AMI
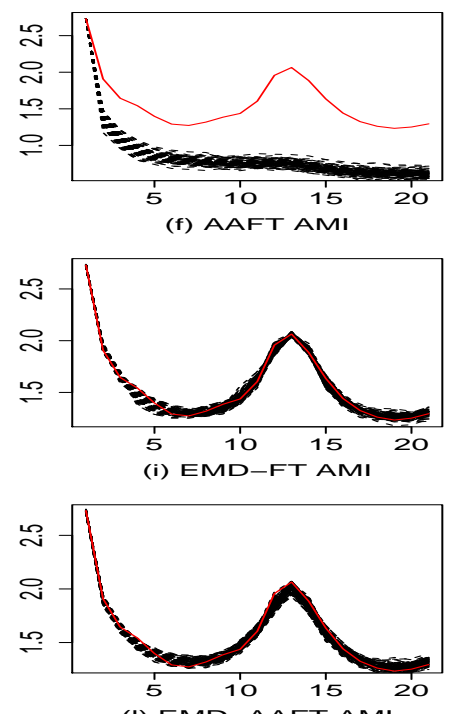

(1) EMD-AAFT AMI

Figure C.6: Surrogates generated from the real-world time series composed of atmospheric concentrations of $\mathrm{CO} 2$. The left side presents the original time series (red continuous line) and its surrogates (dashed lines). In the middle and right side, it is presented the ACF and AMI plots.

results were summarized in Table C.1, in which letters A and R mean the null hypothesis was accepted or rejected, respectively.

According to Table C.1 the surrogates generated by Theiler's FT and AAFT were significantly different from the original time series in most situations, hence the null hypothesis was rejected for most surrogates. On the other hand, the proposed EMD-FT and EMDAAFT methods provided greater p-values, showing that their surrogates are more similar to the original time series.

By analyzing the results obtained with the synthetic and real-world time series, we conclude the proposed EMD-FT and EMD-AAFT methods provide effective surrogates, which respect the behavior of original time series. This is evident in the presence of a trend, which affects Theiler's methods but not EMD-FT and EMD-AAFT. 

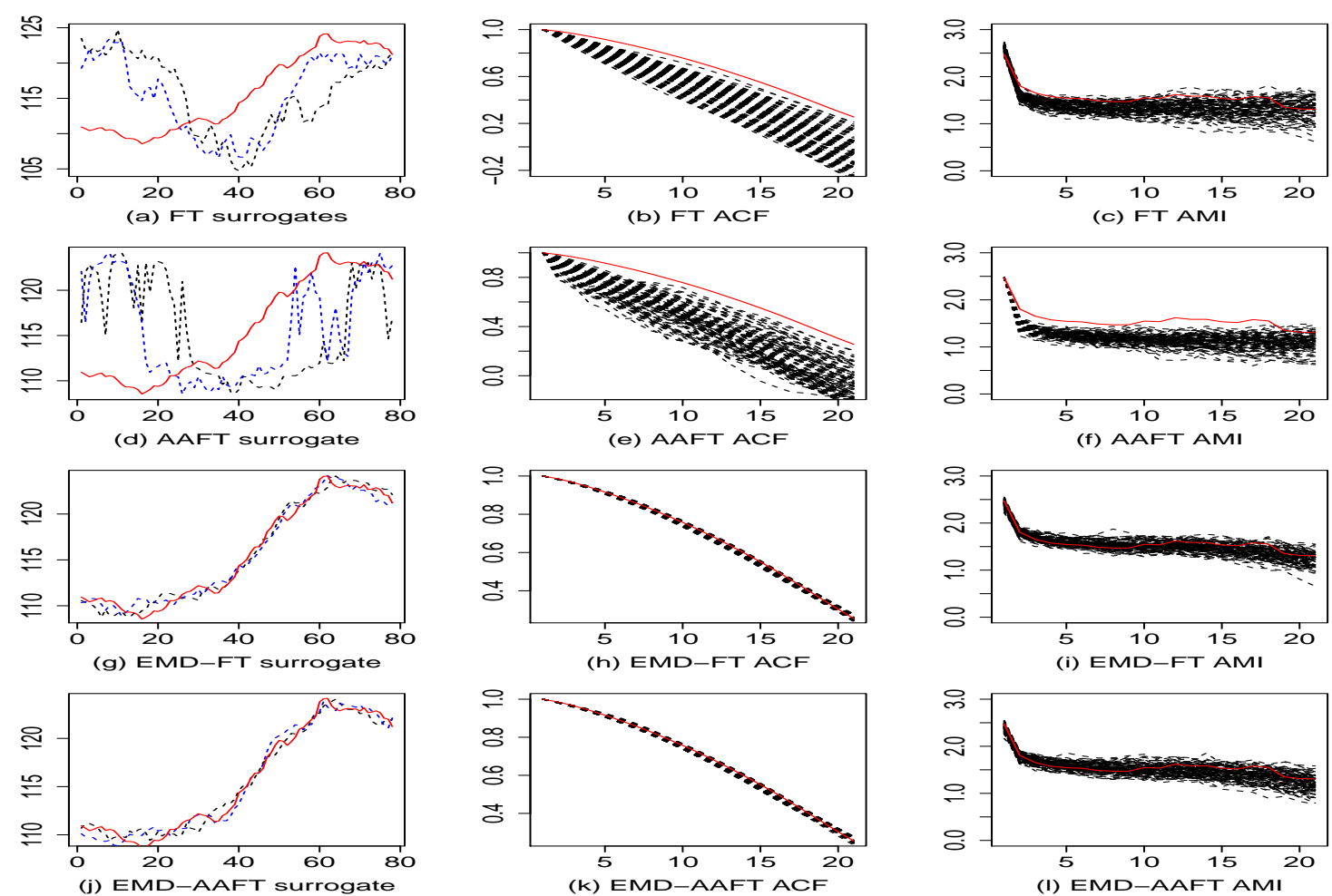

(k) EMD-AAFT ACF

(1) EMD-AAFT AMI

Figure C.7: Surrogates generated from the real-world time series of Dow Jones Utilities Index. The left side presents the original time series (red continuous line) and its surrogates (dashed lines). In the middle and right side, it is presented the ACF and AMI plots.

\section{C.6 Concluding remarks}

In this appendix, we discussed the problem faced by Theiler's FT and AAFT methods when time series present nonstationary behavior. In such situation, surrogates produced by these methods are very different from the original data. By applying statistical methods or even performing a visual inspection on the original and surrogate time series, we cannot state whether they were created from the same process or not.

In order to address this drawback, we proposed two new methods based on Theiler's techniques. The new methods initially decompose the time series into monocomponents that are, in a second step, transformed by either Theiler's FT or AAFT method. As a result, a set of monocomponent surrogates is produced, which are combined with the original time series trend to create the surrogate time series.

Experimental results on synthetic and real-world time series confirmed the proposed methods produced surrogates in accordance to the original data in presence of nonstation- 
Table C.1: Hypothesis test using Average Mutual Information (AMI): FT and AAFT rejected the null hypothesis in 9 out of 12 scenarios, confirming the surrogate series produced are not representative enough; EMD-FT and EMD-AAFT accepted the null hypothesis in all scenarios, confirming they produce more significative surrogate data.

\begin{tabular}{c|ll|ll}
\hline Time Series & FT & AAFT & EMD-FT & EMD-AAFT \\
\hline \hline Sine + Trend & $0(R)$ & $0(R)$ & $0.753(A)$ & $0.013(A)$ \\
White noise + Trend & $0(R)$ & $0(R)$ & $0.601(A)$ & $0.508(A)$ \\
AR(1) + Trend & $0(R)$ & $0(R)$ & $0.565(A)$ & $0.571(A)$ \\
Global Temperature & $0.727(A)$ & $0.942(A)$ & $0.829(A)$ & $0.886(A)$ \\
CO2 & $0(R)$ & $0(R)$ & $0.483(A)$ & $0.412(A)$ \\
Dow Jones & $0.073(A)$ & $0(R)$ & $0.344(A)$ & $0.321(A)$ \\
\hline
\end{tabular}

arity. This is made possible due to the extraction of the series trend, which adds spurious high frequencies. As consequence, the proposed methods support the linear/nonlinear test for stationary and nonstationary time series, what is not possible when directly using Theiler's methods. 


\section{Bibliography}

Abarbanel, H. (1996). Analysis of Observed Chaotic Data. Institute for Nonlinear Science. Springer.

Ahrens, C. (2009). Meteorology Today: An Introduction to Weather, Climate, and the Environment. Brooks/Cole, CengageLearning.

Albertini, M. K. (2012). Adaptação de viés indutivo de algoritmos de agrupamento de fluxos de dados. Tese (Doutorado), Universidade de São Paulo. Tese de doutorado disponível em: http://www.teses.usp.br/teses/disponiveis/55/55134/tde-12062012-085839/ptbr.php. Acessado em: 09 de Setembro de 2013.

Alligood, K. T.; Sauer, T. D.; Yorke, J. A. (1997). Chaos: An Introduction to Dynamical Systems. Springer.

Aminghafari, M.; Cheze, N.; Poggi, J.-M. (2006). Multivariate denoising using wavelets and principal component analysis. Comput. Stat. Data Anal., v.50, p.2381-2398.

Amman, S.; Das, M. (2001). An efficient technique for modeling and synthesis of automotive engine sounds. IEEE Transactions on Industrial Electronics, v.48, n.1, p.225-234.

Anyamba, A.; Tucker, C. (2005). Analysis of Sahelian vegetation dynamics using NOAAAVHRR NDVI data from 1981-2003. Journal of Arid Environments, v.63, n.3, p.596-614. 
Box, G.; Jenkins, G. M.; Reinsel, G. (1994). Time Series Analysis: Forecasting \& Control. Prentice Hall, $3^{a}$ edição.

Brockwell, P. J.; Davis, R. A. (2002). Introduction to Time Series and Forecasting. Springer.

Casdagli, M. (1989). Nonlinear prediction of chaotic time series. Physica D: Nonlinear Phenomena, v.35, n.3, p.335-356.

Chatfield, C. (2004). The Analysis of Time Series: An Introduction. CRC Press LLC.

Chung, P.-J.; Viberg, M.; Mecklenbräuker, C. F. (2010). Broadband ML estimation under model order uncertainty. Signal Process., v.90, p.1350-1356.

Cleveland, W. S. (1993). Visualizing Data. Hobart Press, Summit, New Jersey, U.S.A.

Colominas, M. A.; Gastón Scholotthauer, María E. Torres, P. F. (2012). Noise-assisted EMD methods in action. Advances in Adaptive Data Analysis, v.4, n.4, p.1250025-1 1250025-11.

Darbellay, G.; Vajda, I. (1999). Estimation of the information by an adaptive partitioning of the observation space. IEEE Transactions on Information Theory, v.45, n.4, p.1315-1321.

Darbellay, G. A.; Tichavský, P. (2000). Independent component analysis through direct estimation of the mutual information. Proceedings of 2nd International Workshop on ICA and Blind Source Separation, Helsinki, Finland, p. 69-74. IEEE Signal Processing Society.

de Lima, E.; Andrade, A.; Pons, J.; Kyberd, P.; Nasuto, S. (2006). Empirical mode decomposition: a novel technique for the study of tremor time series. Medical and Biological Engineering and Computing, v.44, p.569-582. 10.1007/s11517-006-0065-x.

Durelli, V. H. S.; Felizardo, K. R.; Delamaro, M. E. (2010). Systematic mapping study on high-level language virtual machines. Virtual Machines and Intermediate Languages, VMIL'10, p. 4:1-4:6, New York, NY, USA. ACM.

Dyba, T.; Kitchenham, B. A.; Jorgensen, M. (2005). Evidence-based software engineering for practitioners. IEEE Software, v.22, p.58-65.

Eckmann, J. P.; Kamphorst, O. S.; Ruelle, D. (1987). Recurrence plots of dynamical systems. Europhysics Letters, v.4, p.973-977. 
Endo, A. T.; da Silva Simão, A. (2010). Formal testing approaches for service-oriented architectures and web services: a systematic review. Relatório Técnico 348, Institute of Mathematic and Computer Sciences, University of São Paulo.

Evidence-based Medicine Working Group (1992). Evidence-based medicine - a new approach to teaching the practice of medicine. The Journal of the American Medical Association (JAMA), v.268, n.17, p.2420-25.

Fensholt, R.; Sandholt, I.; Rasmussen, M. S. (2004). Evaluation of MODIS LAI, fAPAR and the relation between FAPAR and NDVI in a semi-arid environment using in situ measurements. Remote Sensing of Environment, v.91, n.3-4, p.490-507.

Flandrin, P.; Rilling, G.; Goncalves, P. (2004). Empirical mode decomposition as a filter bank. IEEE Signal Processing Letters, v.11, n.2, p.112-114.

Fraser, A. M.; Swinney, H. L. (1986). Independent coordinates for strange attractors from mutual information. Physical Review A, v.33, n.2, p.1134-1140.

Gobron, N.; Belward, A. S.; Pinty, B.; Knorr, W. (2010a). Monitoring biosphere vegetation 1998-2009. Geophysical Research Letters, v.37, n.L15402, p.6.

Gobron, N.; Knorr, W.; Belward, A. S.; Pinty, B. (2010b). Fraction of Absorbed Photosynthetically Active Radiation (FAPAR). Bulletin of the American Meteorological Society, v.91, n.7, p.S50-S51.

Gobron, N.; Pinty, B.; Verstraete, M.; Widlowski, J.-L. (2000). Advanced vegetation indices optimized for up-coming sensors: Design, performance, and applications. IEEE Transactions on Geoscience and Remote Sensing, v.38, n.6, p.2489-2505.

Golyandina, N.; Nekrutkin, V.; Zhigljavsky, A. (2001). Analysis of time series structure: SSA and related techniques, v. 90. Chapman and Hall, Boca Raton, Florida.

Gonzalez, P.; Tucker, C.; Sy, H. (2012). Tree density and species decline in the African Sahel attributable to climate. Journal of Arid Environments, v.78, n.0, p.55-64.

Gonzalez, R. C.; Woods, R. E. (2006). Digital Image Processing. Prentice-Hall, Inc., Upper Saddle River, NJ, EUA, $3^{a}$ edição. 
Graps, A. (1995). An introduction to wavelets. IEEE Computational Science and Engineering, v.2, p.50-61.

Grassberger, P.; Procaccia, I. (1983). Estimation of the Kolmogorov entropy from a chaotic signal. Physical Review A, v.28, n.4, p.2591-2593.

Gruber, P.; Stadlthanner, K.; Böhm, M.; Theis, F.; Lang, E.; Tomé, A.; Teixeira, A.; Puntonet, C.; Saéz, J. G. (2006). Denoising using local projective subspace methods. Neurocomputing, v.69, n.13-15, p.1485-1501. Blind Source Separation and Independent Component Analysis - Selected papers from the ICA 2004 meeting, Granada, Spain, Blind Source Separation and Independent Component Analysis.

Hamilton, J. D. (1994). Time Series Analysis. Princeton University Press.

Han, M.; Liu, Y. (2009). Noise reduction method for chaotic signals based on dual-wavelet and spatial correlation. Expert Systems with Applications, v.36, n.6, p.10060-10067.

Hegger, R.; Kantz, H.; Schreiber, T. (2009). TISEAN 3.0.1: Nonlinear time series analysis. Available at http://www.mpipks-dresden.mpg.de/_tisean/Tisean_3.0.1/index.html.

Heumann, B.; Seaquist, J.; Eklundh, L.; Jonsson, P. (2007). AVHRR derived phenological change in the Sahel and Soudan, Africa, 1982-2005. Remote Sensing of Environment, v.108, n.4, p.385-392.

Hirata, Y.; Katori, Y.; Shimokawa, H.; Suzuki, H.; Blenkinsop, T. A.; Lang, E. J.; Aihara, K. (2008). Testing a neural coding hypothesis using surrogate data. Journal of neuroscience methods, v.172, n.2, p.312-322.

Hsieh, W. W.; Wu, A. (2002). Nonlinear singular spectrum analysis. v.3, p.2819-2824.

Huang, N. E.; Chern, C. C.; Huang, K.; Salvino, L. W.; Long, S. R.; Fan, K. L. (2001). A New Spectral Representation of Earthquake Data: Hilbert Spectral Analysis of Station TCU129, Chi-Chi, Taiwan, 21 September 1999. Bulletin of the Seismological Society of America, v.91, n.5, p.1310-1338.

Huang, N. E.; Shen, Z.; Long, S. R.; Wu, M. C.; Shih, H. H.; Zheng, Q.; Yen, N. C.; Tung, C. C.; Liu, H. H. (1998). The empirical mode decomposition and the Hilbert spectrum for 
nonlinear and non-stationary time series analysis. Royal Society of London Proceedings Series A, v.454, p.903-995.

Ishii, R. P.; Rios, R. A.; Mello, R. F. (2011). Classification of time series generation processes using experimental tools: a survey and proposal of an automatic and systematic approach. International Journal of Computational Science and Engineering, v.6, p.217237.

Jerri, A. (1977). The shannon sampling theorem - its various extensions and applications: A tutorial review. Proceedings of the IEEE, v.65, n.11, p.1565-1596.

Jolliffe, I. T. (2002). Introduction. Principal Component Analysis, Springer Series in Statistics, p. 1-9. Springer New York.

Jr., C. L. W.; Zbilut, J. P. (1994). Dynamical assessment of physiological systems and states using recurrence plot strategies. Journal of Applied Physiology, v.76, n.2, p.965-973.

Kennel, M. B.; Brown, R.; Abarbanel, H. D. I. (1992). Determining embedding dimension for phase-space reconstruction using a geometrical construction. Physical Review A, v.45, n.6, p.3403-3411.

Kirchgässner, G.; Wolters, J. (2007). Introduction to Modern Time Series Analysis. Springer, 1 edição.

Kitchenham, B.; Brereton, O. P.; Budgen, D.; Turner, M.; Bailey, J.; Linkman, S. (2009). Systematic literature reviews in software engineering - a systematic literature review. Information and Software Technology, v.51, n.1, p.7-15. Special Section - Most Cited Articles in 2002 and Regular Research Papers.

Kopsinis, Y.; McLaughlin, S. (2009). Development of EMD-Based Denoising Methods Inspired by Wavelet Thresholding. IEEE Transactions on Signal Processing, v.57, n.4, p.1351-1362.

Kraskov, A.; Stögbauer, H.; Grassberger, P. (2004). Estimating mutual information. Physical review E: Statistical, nonlinear, and soft matter physics, v.69, n.6 Pt 2.

Kroese, D. P.; Taimre, T.; Botev, Z. I. (2011). Handbook of Monte Carlo Methods. John Wiley and Sons, New York. 
Lee, T.; Ouarda, T. B. M. J. (2012). An emd and pca hybrid approach for separating noise from signal, and signal in climate change detection. International Journal of Climatology, v.32, p.624-634.

Lepers, E.; Lambin, E. F.; Janetos, A. C.; DeFries, R.; Achard, F.; Ramankutty, N.; Scholes, R. J. (2005). A synthesis of information on rapid land-cover change for the period 19812000. BioScience, v.55, n.2, p.115-124.

Liu, Y.; Liao, X. (2011). Adaptive chaotic noise reduction method based on dual-lifting wavelet. Expert Syst. Appl., v.38, p.1346-1355.

Lucio, J. H.; Valdes, R.; Rodriguez, L. R. (2012). Improvements to surrogate data methods for nonstationary time series. Physical review. E, Statistical, nonlinear, and soft matter physics, v.85, n.5-2, p.056202-1-19.

Macciotta, N. P.; Cappio-Borlino, A.; Pulina, G. (2000). Time series autoregressive integrated moving average modeling of test-day milk yields of dairy ewes. Journal of dairy science, v.83, n.5, p.1094-103.

Mahecha, M. D.; Fürst, L. M.; Gobron, N.; Lange, H. (2010). Identifying multiple spatiotemporal patterns: A refined view on terrestrial photosynthetic activity. Pattern Recognition Letters, v.31, n.14, p.2309-2317.

Maiwald, T.; Mammen, E.; Nandi, S.; Timmer, J. (2008). Surrogate data - a qualitative and quantitative analysis. Dahlhaus, R.; Kurths, J.; Maass, P.; Timmer, J., editores, Mathematical Methods in Signal Processing and Digital Image Analysis, v. 27 de Understanding Complex Systems, p. 41-74. Springer Berlin / Heidelberg.

Mañé, R. (1980). On the dimension of the compact invariant sets of certain nonlinear maps. Springer.

Marsland, S.; Shapiro, J.; Nehmzow, U. (2002). A self-organising network that grows when required. Neural Networks, v.15, p.1041-1058.

Marwan, N.; Romano, M.; Thiel, M.; Kurths, J. (2007). Recurrence plots for the analysis of complex systems. Physics Reports, v.438, n.5-6, p.237-329. 
Mello, R. F. (2009). Sistemas dinâmicos e técnicas inteligentes para a predição de comportamento de processos: Uma abordagem para otimização de escalonamento em grades computacionais. Tese de livre docência disponível em: http://www.teses.usp.br/teses/disponiveis/livredocencia/55/tde-17082011173637/pt-br.php. Acessado em: 04 de Setembro de 2013.

Meyer, M.; Stiedl, O. (2006). Fractal rigidity by enhanced sympatho-vagal antagonism in heartbeat interval dynamics elicited by central application of corticotropin-releasing factor in mice. Journal of mathematical biology, v.52, n.6, p.830-874.

Moon, T.; Weissman, T. (2009). Universal FIR MMSE filtering. IEEE Transactions on Signal Processing, v.57, n.3, p.1068-1083.

Morettin, P. A.; Toloi, C. M. C. (2004). Análise de Séries Temporais. Editora Edgard Blücher Ltda., São Paulo.

Nakamura, T.; Small, M. (2005). Small-shuffle surrogate data: Testing for dynamics in fluctuating data with trends. Physical Review E, v.72, p.056216.1 - 056216.6.

Nakamura, T.; Small, M.; Hirata, Y. (2006). Testing for nonlinearity in irregular fluctuations with long-term trends. Physical Review E, v.74, p.026205.1 - 026205.8.

Nounou, M. N. (2006). Multiscale finite impulse response modeling. Engineering Applications of Artificial Intelligence, v.19, n.3, p.289-304.

Papana, A.; Kugiumtzis, D. (2008). Evaluation of mutual information estimators on nonlinear dynamic systems. Complex Phenomena in Nonlinear Systems, v.11, n.2, p.225-232.

Petersen, K.; Feldt, R.; Mujtaba, S.; Mattsson, M. (2008). Systematic Mapping Studies in Software engineering. EASE '08: Proceedings of the 12th International Conference on Evaluation and Assessment in Software Engineering.

Provanzale, A.; Smith, L. A.; Vio, R.; Murante, G. (1992). Distinguishing between lowdimensional dynamics and randomness in measured time series. Physica D: Nonlinear Phenomena, v.58, n.1-4, p.31-49.

Rienecker, M. M.; Dee, D.; Woollen, J.; Compo, G. P.; Onogi, K.; Gelaro, R.; Bosilovich, M. G.; daSilva, A.; Pawson, S.; Schubert, S.; Suarez, M.; Barker, D.; Kamahori, H.; 
Kistler, R.; Saha, S. (2012). Atmospheric reanalyses-recent progress and prospects for the future. a report from a technical workshop, april 2010. Relatório técnico, NASA Goddard Space Flight Center; Greenbelt, MD, United States. Doc. Id. 20120014167.

Rios, R. A.; Mello, R. F. (2012). A systematic literature review on decomposition approaches to estimate time series components. Journal of Computer Science, v.11, p.31-46.

Rios, R. A.; Mello, R. F. (2013). Improving time series modeling by decomposing and analyzing stochastic and deterministic influences. Signal Processing, v.93, n.11, p.3001-3013.

Rowhani, P.; Linderman, M.; Lambin, E. F. (2011). Global interannual variability in terrestrial ecosystems: sources and spatial distribution using MODIS-derived vegetation indices, social and biophysical factors. International Journal of Remote Sensing, v.32, n.19, p.5393-5411.

Schoellhamer, D. H. (2001). Singular spectrum analysis for time series with missing data. Geophysical Research Letters, v.28, n.16, p.3187-3190.

Schreiber, T.; Schmitz, A. (1996). Improved surrogate data for nonlinearity tests. Phys. Rev. Lett., v.77, p.635-638.

Shannon, C. E. (2001). A mathematical theory of communication. SIGMOBILE Mob. Comput. Commun. Rev., v.5, n.1, p.3-55.

Shoukat Choudhury, M. A. A.; Shah, S. L.; Thornhill, N. F. (2008). Diagnosis of process nonlinearities and valve stiction - Data driven approaches. Berlin: Springer.

Shumway, R. H.; Stoffer, D. S. (2006). Time Series Analysis and Its Applications: With R Examples (Springer Texts in Statistics). Springer, $2^{a}$ edição.

Small, M.; Tse, C. (2003). Detecting determinism in time series: the method of surrogate data. IEEE Transactions on Circuits and Systems I: Fundamental Theory and Applications, v.50, n.5, p.663-672.

Takens, F. (1980). Detecting strange attractors in turbulence. Dynamical Systems and Turbulence, p. 366-381. Springer. 
Theiler, J.; Eubank, S.; Longtin, A.; Galdrikian, B.; Doynefarmer, J. (1992). Testing for nonlinearity in time series: the method of surrogate data. Physica D: Nonlinear Phenomena, v.58, n.1-4, p.77-94.

Tohru, I.; Kazuyuki, A. (1997-05-25). On dimension estimates with surrogate data sets. IEICE transactions on fundamentals of electronics, communications and computer sciences, v.80, n.5, p.859-868.

Torgo, L. (2010). Data Mining with R: Learning with Case Studies. Chapman \& Hall/CRC, 1st edição.

Tormene, P.; Giorgino, T.; Quaglini, S.; Stefanelli, M. (2009). Matching incomplete time series with dynamic time warping: an algorithm and an application to post-stroke rehabilitation. Artificial Intelligence in Medicine, v.45, p.11-34.

Tsakalozos, N.; Drakakis, K.; Rickard, S. (2012). A formal study of the nonlinearity and consistency of the empirical mode decomposition. Signal Processing, v.92, n.9, p.1961 1969.

Tzagkarakis, G.; Papadopouli, M.; Tsakalides, P. (2007). Singular spectrum analysis of traffic workload in a large-scale wireless lan. Proceedings of the 10th ACM Symposium on Modeling, analysis, and simulation of wireless and mobile systems, MSWiM '07, p. 99-108, New York, NY, USA. ACM.

Wangler, B.; Backlund, A. (2005). Information systems engineering: What is it? CAiSE Workshops (2), p. 427-437.

Whitney, H. (1936). Differentiable manifolds. The Annals of Mathematics, v.37, n.3, p.645680.

Wu, Z. H.; Huang, N. E. (2004). A study of the characteristics of white noise using the empirical mode decomposition method. Proc. R. Soc. Lond. A, v.460, p.1597-1611.

Zampa, P.; Arnost, R. (2002). A new approach to system structure definition. 2002 IEEE International Conference on Systems, Man and Cybernetics, v. 7, p. 5.

Zbilut, J. P.; Jr., C. L. W. (1992). Embeddings and delays as derived from quantification of recurrence plots. Physics Letters A, v.171, n.3-4, p.199-203. 